\title{
Biomimetic Oscillating Foil Propulsion to Enhance Underwater Vehicle Agility and Maneuverability
}

by

\author{
Stephen Carl Licht
}

Submitted in partial fulfillment of the requirements for the degree of Doctor of Philosophy in Oceanographic and Mechanical Engineering at the

MASSACHUSETTS INSTITUTE OF TECHNOLOGY and the WOODS HOLE OCEANOGRAPHIC INSTITUTION

June 2008

(C) Stephen C. Licht, 2008. All rights reserved. The author hereby grants to MIT and WHOI permission to reproduce and to distribute publicly paper and electronic copies of this thesis document in whole or in part in any medium now known or hereafter created.

Author

Joint Program in Oceanography/Applied Ocean Science \& Engineering February 27, 2008

Certified by<smiles>[CH]1CC1</smiles>

Michael S. Triantafyllou Prnfecser of Merhaniral \& Ocean Engineering, MIT Thesis Supervisor

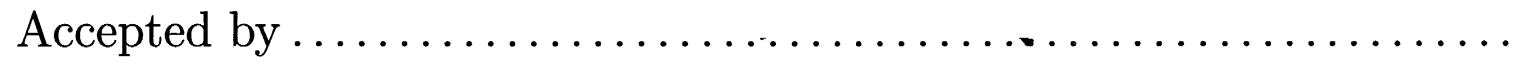

Lallit Anand

Chair, Mechanjcal Engineering Gradyate Committee

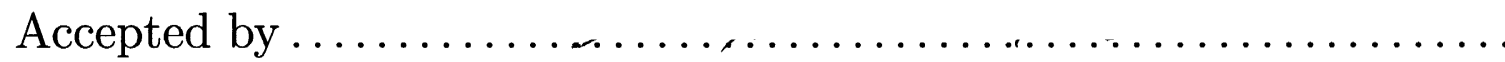

Alexandra Techet

Chair, Joint Committee for Applied Ocean Science \& Engineering 


\title{
Biomimetic Oscillating Foil Propulsion to Enhance
}

\section{Underwater Vehicle Agility and Maneuverability}

by

\author{
Stephen Carl Licht
}

\author{
Submitted to the Joint Program in Oceanography/Applied Ocean Science \& \\ Engineering \\ on February 27, 2008, in partial fulfillment of the \\ requirements for the degree of \\ Doctor of Philosophy in Oceanographic and Mechanical Engineering
}

\begin{abstract}
Inspired by the swimming abilities of marine animals, this thesis presents "Finnegan the RoboTurtle", an autonomous underwater vehicle (AUV) powered entirely by four flapping foils. Biomimetic actuation is shown to produce dramatic improvements in AUV maneuvering at cruising speeds, while simultaneously allowing for agility at low speeds. Using control algorithms linear in the modified Rodrigues parameters to support large angle maneuvers, the vehicle is successfully controlled in banked and twisting turns, exceeding the best reported AUV turning performance by more than a factor of two; a minimum turning radius of $0.7 B L$, and the ability to avoid walls detected $>1.8 \mathrm{BL}$ ahead, are found for cruising speeds of $0.75 \mathrm{BL} / \mathrm{s}$, with a maximum heading rate of $40^{\circ} / \mathrm{s}$ recorded.

Observations of "Myrtle", a 250kg Green sea turtle (Chelonia mydas) at the New England Aquarium, are detailed; along with steady swimming, Myrtle is observed performing $180^{\circ}$ level turns and rapidly actuating pitch to control depth and speed. Limb kinematics for the level turning maneuver are replicated by Finnegan, and turning rates comparable to those of the turtle are achieved. Foil kinematics which produce approximately sinusoidal nominal angle of attack trace are shown to improve turning performance by as much as $25 \%$; the effect is achieved despite limited knowledge of the flow field. Finally, tests with a single foil are used to demonstrate that biomimetically inspired inline motion can allow oscillating foils utilizing a power/recovery style stroke to generate as much as $90 \%$ of the thrust from a power/power stroke style motion.
\end{abstract}

Thesis Supervisor: Michael S. Triantafyllou

Title: Professor of Mechanical \& Ocean Engineering, MIT 


\section{Acknowledgments}

Thank you first to Michael Triantafyllou and Franz Hover, without whose vision and direction "Finnegan" would never have made it to the drawing board, let alone into the pool. Thank you also to Jean-Jacques Slotine, Mark Grosenbaugh, and George Lauder for graciously lending their time and advice while serving on my committee, and to Alex Techet for chairing my defense and providing wise counsel along the way.

The staff and volunteers at the New England Aquarium, especially Sherrie Floyd and Don Stark, were unfailingly welcoming and helpful in my work with Myrtle, and I owe them a debt of gratitude.

I would not have had strength and perseverance needed without the support of my family, and so I dedicate this thesis to my parents, to my wife, and to my daughter, Abigail. 


\section{Contents}

1 Introduction $\quad 27$

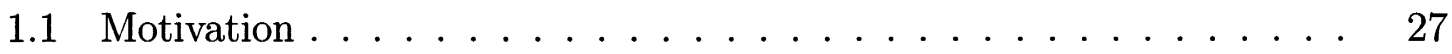

1.2 Background and Literature Review . . . . . . . . . . . . . . 28

1.3 Chapter Preview .................... 34

2 Vehicle Design and Open Loop Performance $\quad 37$

2.1 Introduction . . . . . . . . . . . . . . . . 37

2.2 Background: Biomimetic Vehicles in the Literature . . . . . . . . 37

2.3 Vehicle Design Details . . . . . . . . . . . . . . 39

2.3 .1 Foil Actuators . . . . . . . . . . . . . . 40

2.3.2 Power Supply . . . . . . . . . . . . . . . . . 42

2.3.3 Processors and Inter-Processor Communication . . . . . . . 43

2.3.4 Navigation Sensors . . . . . . . . . . . . . . . 44

2.3.5 Vehicle Configuration and Dimensions ......... 45

2.4 Foil Kinematics . . . . . . . . . . . . . . . 50

2.5 Code Theory of Operation . . . . . . . . . . . . . 52

2.5.1 State Estimation ............... 56

2.6 Practical Design Tools for Foil Actuation . . . . . . . . . . . . . 57

2.6.1 Application to Finnegan Actuator Design . . . . . . . . . . 62

2.7 Open Loop Vehicle Testing . . . . . . . . . . . . . . . . . . 63

2.7.1 Surge, Sway, Heave and Yaw Kinematics . . . . . . . . . . 64

2.7.2 Performance Results . . . . . . . . . . . . . . . . 66

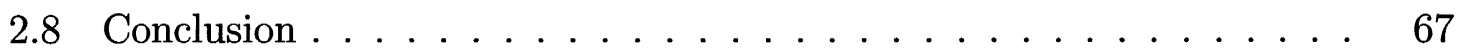


3 Control and Maneuvering with Harmonically Oscillating Foils 69

3.1 Introduction . . . . . . . . . . . . . . . . . . . 69

3.2 Background $\ldots \ldots \ldots \ldots \ldots \ldots \ldots \ldots \ldots \ldots \ldots \ldots \ldots \ldots$

3.3 Attitude Regulation using the Modified Rodrigues Parameters _ . . . 73

3.3.1 Foil Force Vector Command . . . . . . . . . . . . . . 78

3.4 Foil Force Vectoring . . . . . . . . . . . . . . . . . . . . . 81

3.4 .1 Hovering . . . . . . . . . . . . . . . . . . . 87

3.4.2 Tuning and Initial Maneuvering Experiments Under Joystick Control . . . . . . . . . . . . . . . . . . 89

3.5 Maneuvering Experiments $\ldots \ldots \ldots \ldots$. . . . . . . . 92

3.5.1 Level Turning . . . . . . . . . . . . . . . . . . . . 95

3.5.2 Banked Turns . . . . . . . . . . . . . . . . . . 99

3.5.3 "Twisting" Turns . . . . . . . . . . . . . . . . . . . . . . 108

3.6 Conclusion . . . . . . . . . . . . . . . . . . . . . . . . . . . 112

4 Sea Turtle Maneuvering $\quad 117$

4.1 Introduction . . . . . . . . . . . . . . . . . 117

4.2 Experimental Procedure . . . . . . . . . . . . . . . . . . . 118

4.3 General Observations on Behavior and Limb Kinematics . . . . . . 120

4.4 Detailed Analysis of Selected Behaviors . . . . . . . . . . . . . . . 120

4.4 .1 Rapid Pitching . . . . . . . . . . . . . . . . . . . 121

4.4 .2 Shallow descent . . . . . . . . . . . . . . . . . . 124

4.4.3 Level Turning at Low Speed . . . . . . . . . . . . . . . 126

4.4.4 Straight Line Swimming . . . . . . . . . . . . . . . 134

4.5 Discussion of Results . . . . . . . . . . . . . . . . . . 138

4.5.1 Animal Motivation and Generalizability . . . . . . . . . 138

4.5 .2 Maneuvering Strokes . . . . . . . . . . . . . . . . . . 139

4.5 .3 Steady Swimming . . . . . . . . . . . . . . . . . 140

4.6 Conclusion . . . . . . . . . . . . . . . . . . . . . . . . . . . 141 
5.1 Introduction . . . . . . . . . . . . . . . . . . . . 143

5.2 Adaptation of Turtle Level Turning Kinematics _ . . . . . . . . 145

5.2.1 Combined Lift and Drag Foil Actions. . . . . . . . . . . . 153

5.2.2 Lift Based (Outside) Foil Action Alone. . . . . . . . . . . . . 155

5.2 .3 Combined Foil Action with Sinusoidal $\alpha(t) . \ldots \ldots \ldots$

5.3 Results . . . . . . . . . . . . . . . . . . . . 160

5.3.1 Combined Lift and Drag Foil Actions. . . . . . . . . 160

5.3.2 Lift Based (Outside) Foil Action Alone. . . . . . . . . . . 167

5.3.3 Combined Foil Action with Sinusoidal $\alpha(t) . \ldots \ldots \ldots$

5.4 Discussion . . . . . . . . . . . . . . . 175

5.5 Conclusion . . . . . . . . . . . . . . . . . . . 181

6 Bioinspired Inline Motion in 2-D Oscillating Foils 183

6.1 Introduction . . . . . . . . . . . . . . . . . . . . 183

6.2 Oscillating Foil Characterization . . . . . . . . . . . . . . . 184

6.3 Methods . . . . . . . . . . . . . . . . . . . . 185

6.3.1 Coordinate Definition . . . . . . . . . . . . . . 185

6.3.2 Adaptation of Kinematic Parameters . . . . . . . . . . . 185

6.3.3 Parametric Study . . . . . . . . . . . . . . . . . . . . . . 191

6.4 Results and Discussion . . . . . . . . . . . . . . . . . 195

6.4.1 Lift and Thrust for Symmetric vs. Asymmetric Kinematics . . 195

6.4.2 Lift and Thrust During Upstroke and Downstroke . . . . . . 198

6.4.3 Rotation of Peak Force Direction with Increasing Inline Motion 203

6.5 Conclusion . . . . . . . . . . . . . . . . . . . 205

$\begin{array}{lll}7 \text { Conclusion } & 207\end{array}$

7.1 Harmonically Oscillating Foils . . . . . . . . . . . . . . . 207

7.2 Sea Turtle Maneuvering and Biomimetic Foil Kinematics . . . . . . 208 


\section{List of Figures}

2-1 Dual Housing Actuator Design. The stationary cylinder contains a DC brush motor driving the rotating cylinder about the "roll" axis. The rotating cylinder contains a smaller motor which drives the "twist" axis. 40

2-2 Vehicle dimensions with fairing installed. This trim/fairing configuration is used for all trials detailed in Chapter 2. The vehicle is stable in pitch and roll in this configuration, with the nose and tail of the fairing filled with shaped buoyancy foam. The fairing is flooded, with components individually waterproofed. . . . . . . . . . . .

2-3 Vehicle sensor and construction detail. Vehicle is attitude unstable in this configuration, which is used for all trials detailed in Chapter 3. . 46

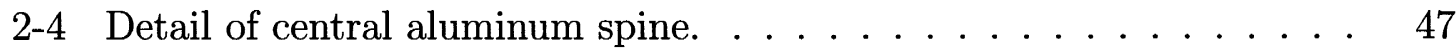

2-5 Detail of available buoyancy foam sections. . . . . . . . . . 48

2-6 Vehicle components during assembly at the MIT Towing Tank. . . . . 48

2-7 Block diagram illustrating flow of underlying operational code. . . . . 52

2-8 Annotated example of mission script file. . . . . . . . . . 55

2-9 Towtank Actuator Test Apparatus _ . . . . . . . . . . . 57

2-10 Contours of thrust coefficient, $C_{T}$, for a harmonically rolling and twisting rigid foil with roll amplitude $\phi_{0}=60^{\circ}$, resulting in $h_{0} / c=4.8$ at $r_{0.7}$. Foil span is $0.4 \mathrm{~m}$, with constant NACA-012 cross section, chord length $0.1 \mathrm{~m}$. (Compare to vehicle foil, partially flexible with $0.4 \mathrm{~m}$ span, and maximum chord or $0.1 \mathrm{~m}$.) Data from experiments performed by Polidoro in [53] and previously published by Licht [43]. . . . . . . . 
2-11 Estimation of Vehicle Speed Limits with Varying $C_{D}$ : Power and acceleration limits. Separate curves represent scenarios with different vehicle drag coefficients, $C_{D}$, and hence different foil thrust requirements. The speed at which the curves cross the constant angular acceleration and power output limits indicates an estimate of the vehicle top speed for the corresponding vehicle $C_{D}$. All curves are extrapolated from experimental data points in Polidoro [53], where vehicle actuators where tested at towed speed of $0.5 \mathrm{~m} / \mathrm{s} \ldots \ldots \ldots \ldots$

2-12 Estimation of Vehicle Speed Limits with Varying $C_{D}$ :Torque-speed requirements with flow speed increase. Curve I represents constant power output limits. Curve II represents nominal torque-speed curve. . . . .

2-13 Foil orientation and force vectoring to actuate surge, sway, heave and yaw in open loop. . . . . . . . . . . . . . . . .

3-1 Screen capture from video of vehicle performing banked turn in the MIT Alumni Pool . . . . . . . . . . . . . . . . .

3-2 Screen capture from underwater video of vehicle performing banked turn in the MIT Alumni Pool . . . . . . . . . . . . .

3-3 The vehicle trim configuration is changed to reduce attitude stability by rearranging the buoyancy foam. The nose and tail cones are removed, and replaced with buoyancy located below the vehicle center of gravity. Without the nose cones, removal of the flooded fairing eliminates a large volume of captured water from from the effective mass of the vehicle, further promoting maneuverability. The vehicle unactuated equilibrium position with the fairing is illustrated in Figure 3-4. The vehicle unactuated equilibrium position in unstable trim configuration without the fairing is shown in Figure 3-5 . . . . . . . . . .

3-4 Vehicle unactuated equilibrium position in stable trim configuration (i.e. with nose cones). . . . . . . . . . . . . . . 
3-5 Vehicle unactuated equilibrium position in unstable trim configuration (i.e. without nose cones). The buoyancy used to replace the nose cone buoyancy volume can be seen running the lenght of the vehicle on either side of the DVL (the orange transducer faces indicate the central location of the upturned DVL.) . . . . . . . . . . .

3-6 Foil mean position for hovering tasks. If $\phi_{0}$ and $\theta_{0}$ are identical for all four foils, with $\theta_{\beta}=0$, the mean forces from the fore and aft foils are directly opposed, resulting in zero nominal force and torque on the body. 78

3-7 Foil mean position for cruising. If $\phi_{0}$ and $\theta_{0}$ are identical for all four foils, with $\theta_{\beta}=0$, the mean forces from the fore and aft foils all contribute to drive the vehicle in, with zero nominal heave and sway force, and zero torque on the body. . . . . . . . . . . .

3-8 Single housing actuator design. Foil shaft protrudes through a slot in the Lexan box housing all components. Seal was accomplished with a flexible bellows. This design was used for testing in the MIT water tunnel where foil motion was restricted by flow section, but the range of motion in roll was deemed to be too limited for use on the vehicle.

3-9 Mean $C_{L}$ v. twist bias $\theta_{\text {beta }}$ Data from experiments performed by Flo-

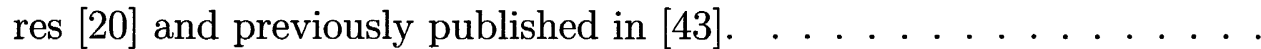

3-10 Effect of twist bias $\theta_{\beta}$ on $C_{L}$ and $C_{T}$. Data from experiments performed by Flores [20] and previously published in [43] . . . . . . . . . . . .

3-11 Joystick Mapping . . . . . . . . . . . . . . . . . . . . . . 9 90

3-12 Vehicle attitude during joystick controlled banked turn. . . . . . . . . 91

3-13 Vehicle attitude during joystick controlled level turn. . . . . . . . . . 92

3-14 Finnegan best turning performance at speed as compared to turning radii of vehicles with various conventional thruster/control surface combinations, as well as sealion (Zalophus californianus and Eumatopias jubatus ) turning rates (see Table 3.1 for values and sources). 
3-15 Screen capture from underwater video footage showing vehicle during test at the MIT Alumni Pool. As shown, the vehicle is in the unstable

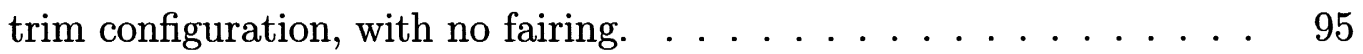

3-16 Top view of tracklines for all turns discussed above. All tracklines are translated and rotated such that the track passes through the origin at the point where the turn is initiated, with velocity in the $+\mathrm{X}$ direction. The level turn (A) is in green, banked turns $(\mathbf{B}, \mathbf{C}, \mathbf{D}, \mathbf{E})$ are in blue, and twisting turns $(\mathbf{F}, \mathbf{G}, \mathbf{H})$ in red. . . . . . . . . . .

3-17 Visualization of a level turn. (Case A) Vehicle and foil positions and orientations are shown at one second intervals, with lines tracing the

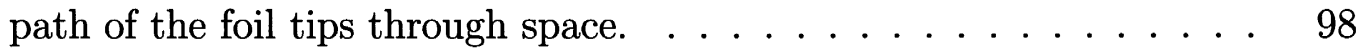

3-18 Top view of a level turn. (Case A) Vehicle and foil positions and orientations are shown at one second intervals. . . . . . . . . . . 99

3-19 Vehicle position and attitude throughout level turn. (Case A) $\ldots 100$

3-20 Vehicle velocities and angular rates throughout level turn. (Case A) . 101

3-21 Roll angle $\phi(t)$ (blue), pitch angle $\theta(t)$ (green), and nominal angle of attack $\alpha(t)$ (red) resulting from foil motion, vehicle velocity, and vehicle rotation, throughout level turn. (Case A). . . . . . . . . . . . . 102

3-22 Visualization of a banked turn. (Case B) Vehicle and foil positions and orientations are shown at one second intervals, with lines tracing the path of the foil tips through space. . . . . . . . . . . . . 103

3-23 Top view of a banked turn. (Case B) Vehicle and foil positions and orientations are shown at one second intervals. . . . . . . . . . . . 104

3-24 Vehicle position and attitude throughout banked turn. (Case B) . . . 105

3-25 Visualization of a banked turn . (Case D) Vehicle and foil positions and orientations are shown at one second intervals, with lines tracing the path of the foil tips through space. . . . . . . . . . . . 106

3-26 Top view of continuous banked turn with saturated body pitch actuation . (Case D) Vehicle and foil positions and orientations are shown at one second intervals. . . . . . . . . . . . . . . . . . . . 107 
3-27 Vehicle position and attitude throughout continuous banked turn with saturated body pitch actuation. (Case D) . . . . . . . . . . 108

3-28 Vehicle position and attitude throughout continuous banked turn with saturated body pitch actuation. (Case D) . . . . . . . . . . 109

3-29 Roll angle $\phi(t)$ (blue), pitch angle $\theta(t)$ (green), and nominal angle of attack $\alpha(t)$ (red) resulting from foil motion, vehicle velocity, and vehicle rotation, at the beginning of a continuous banked turn with saturated body pitch actuation. (Case D). . . . . . . . . . . 110

3-30 Visualization of a twisting turn . (Case F). Vehicle and foil positions and orientations are shown at one second intervals, with lines tracing the path of the foil tips through space. . . . . . . . . . . 111

3-31 Top view of a banked turn . (Case F). Vehicle and foil positions and orientations are shown at one second intervals. . . . . . . . . . . . . 112

3-32 Vehicle position and attitude throughout twisting turn. (Case F) . . 113

3-33 Roll angle $\phi(t)$ (blue), pitch angle $\theta(t)$ (green), and nominal angle of attack $\alpha(t)$ (red) resulting from foil motion, vehicle velocity, and vehicle rotation, throughout twisting turn. (Case F). . . . . . . . . . . . . 114

4-1 Front view of rapid pitching maneuver to retrieve food. Myrtle achieved a high pitch angle through a single rapid down- and forward- stroke of her pectoral fins with a high angle of attack to the flow. . . . . . .

4-2 Side view of rapid pitching maneuver in preparation for swim to surface. Myrtle maintained forward speed while pitching upwards, utilizing less foil motion inline with the flow than in Figure 4-1, where the forward motion was halted during a more rapid pitching maneuver.

4-3 Front view, swimming to target ahead and slightly below. Myrtle utilizes a fore-aft rowing stroke with her forelimbs rather than using a lift based stroke which would tend to pitch her up and away from the

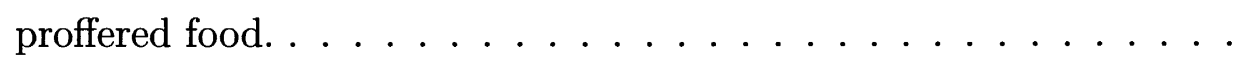


4-4 Side/rear view of swimming to target ahead and slightly below (simultaneous with view shown in Figure 4-4. The nearly vertical state of the pectoral fin surface is particularly evident from this view. (The frames at $\mathrm{t}=0.83$ and $\mathrm{t}=1$ second were obscured by a fish swimming in front of the camera.) . . . . . . . . . . . . .

4-5 Part of a representative level turn using rear limb rapid paddling stroke and inline motion of outside forelimb. The inside forelimb is held level at first, then joins the outside forelimb in a fore-act rowing motion to

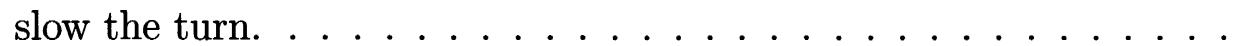

4-6 Front view of part of representative level turn with active participation of both forelimbs. Frames start midway through the turn: $t=0$ sec corresponds to the end of the first downstroke of the outside forelimb

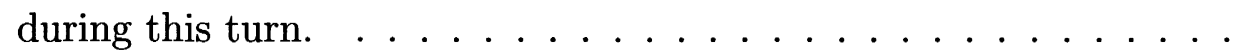

4-7 Side view of representative level turn with active participation of both forelimbs (simultaneous with Figure 4-6. This view highlight the extension of the outside forelimb as it is thrown forward just as the downstroke is initiated. . . . . . . . . . . . . . .

4-8 Limb tracking data for turn with active participation of both forelimbs shown in Figure 4-6 and Figure 4-7. Blue, green and red correspond to the foil tip, leading edge and trailing edge at join between upper and lower limb sections, respectively. Y-scale is normalized pixel location on the side camera view, shown in inset pictures. . . . . . . . .

4-9 Side view of representative steady swimming stroke. . . . . . . . . 135

4-10 Representative frame with tracking data used to find steady swimming velocity. Velocity of extreme front and rear of carapace shown for comparison with body length indicated by white line. . . . . . . .

4-11 Tracking data for foil tip (blue), leading edge (green) and trailing edge (red) at join between upper and lower forelimb. Pixel data is used to determine downstroke and upstroke duration simultaneously with speed determination from Figure 4-10. . . . . . . . . . . . . . 137 
4-12 Representative frame with tracking data used to find steady swimming stroke durations. Points tracked are indicated on foil with white circles. 138

5-1 Cartoon showing top view of turtle hindlimb kinematics during kick start of level turning. The motion of the hindlimbs depicted here is not available to the vehicle foils, which are instead held stationary throughout the turn . . . . . . . . . . . . . . . 146

5-2 Vehicle unactuated roll rate response, in stable trim configuration, to repeated disturbance in roll. . . . . . . . . . . . . . . . . . . 146

5-3 Vehicle unactuated pitch rate response, in stable trim configuration, to repeated disturbance in pitch. . . . . . . . . . . . . . . . 147

5-4 Foil kinematics during biomimetic level turn, for forward foils to the inside and outside of the turn, using combined forelimb motion with piecewise sinusoidal motion. Case B . . . . . . . . . . . . . 148

5-5 Foil kinematics during biomimetic level turn, for forward foils to the inside and outside of the turn, using combined forelimb motion. Outside forelimb motion altered for symmetric twist. Case B' . . . . . . . . . 149

5-6 Foil kinematics during biomimetic level turn, for forward foils to the inside and outside of the turn, using outside forelimb action only, and piecewise sinusoidal motion. Case A . . . . . . . . . . . 150

5-7 Foil kinematics during biomimetic level turn, for forward foils to the inside and outside of the turn, using outside forelimb action only. Outside forelimb motion altered for symmetric twist. Case $\mathbf{A}^{\prime} \ldots \ldots$

5-8 Foil kinematics during biomimetic level turn, for forward foils to the inside and outside of the turn, using combined forelimb action, with piecewise sinusoidal $\alpha(t)$ for the outside foil. Case $\mathbf{C} \ldots \ldots \ldots$

5-9 Visualization of foil kinematics from start of biomimetic level turn using combined forelimb action.. Top and bottom views are paired at 0.33 second intervals. Case B . . . . . . . . . . . . 160 
5-10 Visualization of foil kinematics from start of biomimetic level turn using outside forelimb action only. Top and bottom views are paired at 0.33 second intervals. Case $\mathbf{A} \ldots \ldots \ldots 1$

5-11 Top view of vehicle motion during representative biomimetic level turning trial, using both inside and outside forward foil for thrust. Case

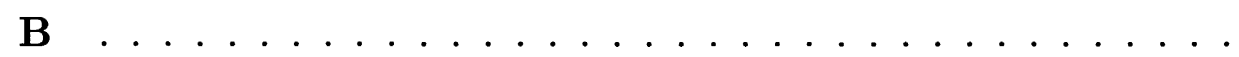

5-12 Cartoon for visualization of body and foil motion through a portion of a biomimetic level turn, with virtual camera angle and time interval designed to allow direct qualitative comparison to turtle behavior shown in Figure 5-13. Case B . . . . . . . . . . . .

5-13 Screen captures from video of turtle performing a representative level turn with active participation of both forelimbs, repeated for clarity. This view highlights the extension of the outside forelimb as it is thrown forward just as the downstroke is initiated. . . . . . . . . . . . . . . . 164

5-14 Heading and heading rate for three cases: with and without inside forelimb rowing motion, and with forelimb rowing motion using sinusoidal angle of attack profile instead of simple sinusoidal kinematics. Vertical lines indicate time at which foil crosses midpoint of roll for downstrokes

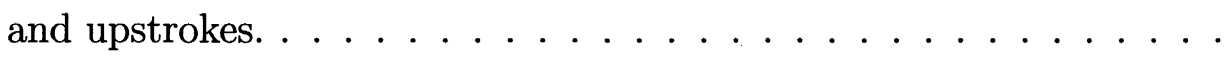

5-15 Heading and heading rate for three cases: with and without inside forelimb rowing motion, and with forelimb rowing motion using sinusoidal angle of attack profile instead of simple sinusoidal kinematics. Vertical lines indicate time at which foil crosses midpoint of roll for downstrokes

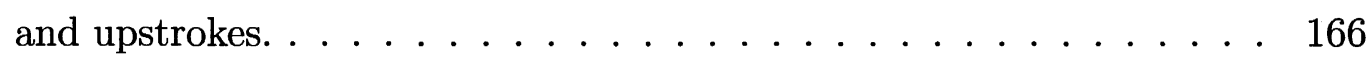


5-16 Body roll response to biomimetic level turning foil kinematics, for Cases B, B'. and B". Attempting a power stroke on both the upstroke and the downstroke (B') leads to larger roll excursion with minimal turning performance difference than feathering the upstroke (B). Using a power stroke for both, but increasing twist angle from $30^{\circ}$ to $40^{\circ}$ (B") does not increase roll excursion, but results in a drop in turning performance (see Table 5.1). Vertical lines indicate time at which foil crosses midpoint of roll for downstrokes and upstrokes. . . . . . . . . . . . . . . . 167

5-17 Body roll response to biomimetic level turning foil kinematics, for Cases B, B'. and B'. Attempting a power stroke on both the upstroke and the downstroke for the outside foil(B',B') reduces the tendency of the vehicle to pitch up during the level turning maneuver, in comparison to kinematics with a feathered upstroke (B). Vertical lines indicate time at which foil crosses midpoint of roll for downstrokes and upstrokes. .

5-18 Sinusoidal foil kinematics and resulting nominal angle of attack at $70 \%$ span for outside forelimb during biomimetic level turn. The angle of attack profile that would obtain if the body had zero angular velocity $\left(\alpha^{*}\right)$ is indicated with a dotted line. Case B. . . . . . . . . . . .

5-19 Foil kinematics calculated a priori to produce approximately sinusoidal nominal angle of attack profile, with $\alpha_{\max }=40^{\circ}$, at $70 \%$ span of outside forelimb during biomimetic level turn, with resulting nominal angle of attack from average vehicle response during experiments. The angle of attack profile that would obtain if the body had zero angular velocity is indicated as well. Case C. . . . . . . . . . . . . . .

5-20 Foil kinematics calculated a priori to produce approximately sinusoidal nominal angle of attack profile, with $\alpha_{\max }=30^{\circ}$, at $70 \%$ span of outside forelimb during biomimetic level turn, with resulting nominal angle of attack from average vehicle response during experiments. The angle of attack profile that would obtain if the body had zero angular velocity $\left(\alpha^{*}\right)$ is indicated as well. Case $\mathbf{C}^{*}$ 
5-21 Illustration of the dependence of nominal angle of attack profile on spanwise location on foil. Kinematics used are calculated to produce $\alpha_{\text {max }}=40^{\circ}$ at $70 \%$ span (Case C). The dotted lines represent the calculated angles of attack occurring throughout the maneuver at five equally spaced points from root to tip of the foil. (To isolate effect of spanwise location, the effect of vehicle angular rates on angle of attack have been removed.) . . . . . . . . . . . . . . .

5-22 Comparison of heading change after completion of maneuver for variations on the biomimetic level turning kinematics, as given in Table 5.1174

5-23 Comparison of maximum heading rate during maneuver for variations on the biomimetic level turning kinematics, as given in Table $5.1 \ldots 174$

5-24 Top view, vehicle track and vehicle orientation at $t=10$ seconds for a trial where only the outside foil is used to actuate the turn. (Case A)

5-25 Top view, vehicle track and vehicle orientation at $t=10$ seconds for a trial where both foils are used to actuate the turn, with sinusoidal twist angle on the outside foil. (Case B) $\ldots \ldots \ldots \ldots \ldots$

5-26 Top view, vehicle track and vehicle orientation at $t=10$ seconds for a trial where both foils are used to actuate the turn, with sinusoidal angle of attack on the outside foil. (Case C) . . . . . . . . 178

5-27 Body fixed vehicle surge and sway velocity for the baseline case for the three adaptations of the biomimetic level turn, Case $\mathbf{A}, \mathbf{B}$, and $\mathbf{C}$ (see Table 5.1 for comparson of foil kinematics.) . . . . . . . . . . .

5-28 Top view, comparison of turning performance between turns using harmonically oscillating foils and biomimetic turn with both limbs participating, and a sinusoidal angle of attack profile on the outside limb. .

6-1 Foil position with respect to fluid at regular intervals, where advance angle $\theta_{A D V}=70^{\circ}$. Arrow represent relative flow velocity. The nominal angle of attack is shown by the angle between the relative flow and the line extending the centerline of the foil. . . . . . . . . . . 
6-2 Foil position with respect to steadily moving platfrom, at regular intervals, for a downstroke where advance angle $\theta_{A D V}=70^{\circ}$. Arrow represent relative flow velocity. The nominal angle of attack is shown by the angle between the relative flow and the line extending the centerline of the foil. . . . . . . . . . . . . .

6-3 Foil position with respect to steadily moving platfrom, at regular intervals, for a downstroke where advance angle $\theta_{A D V}=100^{\circ}$. Arrow represent relative flow velocity. The nominal angle of attack is shown by the angle between the relative flow and the line extending the cen-

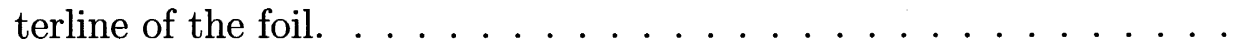

6-4 Foil position with respect to steadily moving platfrom, at regular intervals, for an upstroke following a downstroke where advance angle $\theta_{A D V}=70^{\circ}$. Arrow represent relative flow velocity. The nominal angle of attack is shown by the angle between the relative flow and the line extending the centerline of the foil. . . . . . . . . .

6-5 Foil position with respect to steadily moving platfrom, at regular intervals, for an upstroke following a downstroke where advance angle $\theta_{A D V}=70^{\circ}$. Arrow represent relative flow velocity. The nominal angle of attack is shown by the angle between the relative flow and the line extending the centerline of the foil. . . . . . . . . . .

6-6 View of single foil experimental apparatus showing actuators, sensor and foils mounted to moving platform. . . . . . . . . . . .

6-7 View of single foil experimental apparatus showing position of moving platform on water tank. ..................

6-8 Cartoon of single foil experimental apparatus indicating dimensions as well as direction of positive transverse, inline and twist motion with respect to direction of steady translation. . . . . . . . . . . . 
6-9 Mean lift coefficient, $\overline{C_{L}}$ and mean thrust coefficient, $\overline{C_{T}}$ for asymmetric foil motion with downstroke $\alpha_{\max }=40^{\circ}$, upstroke $\alpha_{\max }=0^{\circ}$, vary Strouhal number and advance angle. Blue: $\overline{C_{T}}$, Red: $\overline{C_{L}}$. Filled symbols indicate $\overline{C_{T}}$ for symmetric motions with no inline motion, and upstroke $\alpha_{\max }=40^{\circ} \ldots \ldots \ldots \ldots \ldots \ldots \ldots \ldots \ldots$

6-10 Comparison of mean lift coefficient, $\overline{C_{L}}$ and mean thrust coefficient, $\overline{C_{T}}$ over each half stroke for asymmetric foil motion, with downstroke $\alpha_{\max }=40^{\circ}$, upstroke $\alpha_{\max }=0^{\circ}$, Strouhal number $=0.4$, with varying advance angle. Filled symbols indicate $\overline{C_{T}}$ for full cycles of symmetric motions with no inline motion and upstroke $\alpha_{\max }=40^{\circ} . \ldots \ldots$

6-11 Phase averaged thrust coefficient, $C_{T}$ and lift coefficient, $C_{L}$ for symmetric foil motion, with downstroke $\alpha_{\max }=40^{\circ}$, upstroke $\alpha_{\max }=40^{\circ}$, and Strouhal number $=0.4, \ldots \ldots \ldots \ldots$

6-12 Phase averaged thrust coefficient, $C_{T}$ and lift coefficient, $C_{L}$ for asymmetric foil motion, with downstroke $\alpha_{\max }=40^{\circ}$, upstroke $\alpha_{\max }=0^{\circ}$, Strouhal number $=0.4$, with no inline motion relative to the steadily translating carriage . . . . . . . . . . . . . .

6-13 Efficiency, $\eta$, of thrust production varying advance angle for foils with downstroke $\alpha_{\max }=40^{\circ}$, upstroke $\alpha_{\max }=0^{\circ}$, for all values of St number tested. Filled symbols indicate $\eta$ for symmetric foil motions, with with downstroke $\alpha_{\max }=40^{\circ}$, upstroke $\alpha_{\max }=40^{\circ}$, and no inline motion, for each value of St number $\ldots \ldots \ldots \ldots \ldots$

6-14 Phase averaged thrust coefficient, $C_{T}$ and lift coefficient, $C_{L}$ for downstroke $\alpha_{\max }=40^{\circ}$, upstroke $\alpha_{\max }=0^{\circ}$, Strouhal number $=0.4$, for four cases with advance angles ranging from the minimum possible (i.e. no relative inline motion) to $\pi / 2$, i.e. no forward motion relative to the water in the center of the downstroke.) . . . . . . . . . .

6-15 Foil position with respect to fluid at regular intervals, where advance angle $\theta_{A D V}=70^{\circ}$. Arrows represent recorded fluid force on foil. . . . 202 
6-16 Foil position with respect to fluid at regular intervals, where advance angle $\theta_{A D V}=100^{\circ}$. Arrows represent recorded fluid force on foil. . . 203

6-17 Foil position with respect to fluid at regular intervals, where advance angle $\theta_{A D V}=100^{\circ}$. Arrows represent recorded fluid force on foil. . . 203 


\section{List of Tables}

2.1 Maximum Surge Velocities $[B L / s]$ vs. Kinematic Parameters . . . 66

2.2 Maximum Yaw Velocities $[\% / s]$ vs. Kinematic Parameters $\ldots . . .67$

2.3 Peak Heave Velocities $(B L / s) \ldots \ldots \ldots \ldots \ldots \ldots$

3.1 Reported turning radius of AUVs with comparison to the sealions $Z a$ lophus californianus and Eumatopias jubatus _. . . . . . . 94

3.2 Baseline Kinematics for Turns Depicted in Figure 3-16. *Value in parenthesis is turning radius based on $180^{\circ}$ turn, for comparison to reported AUV operational turning radii. . . . . . . . . . . . 97

5.1 Summary of Results: Biomimetic Level Turning Maneuvers . . . . . 175 


\section{Chapter 1}

\section{Introduction}

\subsection{Motivation}

Millennia of evolution have produced ever faster, ever more agile, ever more maneuverable underwater predators and prey. The extraordinary abilities of these marine animals have long astounded both the casual observer and the professional naval architect; while we may be able to create vehicles that can swim faster in open water through brute force, the quick starting and nimble turning that allows species as diverse fish, penguins and turtles to survive in the dynamic, unpredictable flows of rivers and surf zones continue to defy imitation. To push the operating range of underwater vehicles, particularly small autonomous robots, into these chaotic margins we must close the performance gap between nature and machine.

The field of biomimetic robotics attempts to observe, adapt and apply the design and behavior of biological examples to improve the performance of man-made devices. This thesis documents the design, creation and successful deployment of "Finnegan", an agile and aggressively maneuvering biomimetic autonomous underwater vehicle. Finnegan is propelled entirely with biologically inspired oscillating foils; as detailed below, biomimetic propulsion in general, and oscillating foils in particular, have been extensively studied as a possible means for improvement of underwater vehicle agility and maneuverability. The objective of the work is to prove the ability of rolling and twisting foils to improve the maneuvering performance of AUVs, as defined by the 
turning radius and turning rate at speed, while simultaneously providing the agility to control six degrees of freedom at low speed in confined space. In developing Finnegan, we take advantage of a growing body of knowledge into the "why?" and "how?" of fish, bird, reptile and mammal swimming to extend the state of the art in underwater vehicles, demonstrating tangible results from a biomimetic approach.

\subsection{Background and Literature Review}

\section{BCF Swimming}

For the purposes of biorobotics research, animal swimming is commonly viewed through the lens of a simplified categorization based on both functional and morphological distinctions, primarily focusing on body-caudal fin (BCF), body-caudal fin transient (BCF transietnt) and median paired fin (MPF) swimming, as in Sfakiotakis [57]. BCF type swimming was the dominant strand of early research into fish biomimetics, strongly encouraged by the so-called Gray's paradox, based on a 1936 article by James Gray [25] which purported to show that the power required to propel a rigid body shaped like a dolphin far exceeded the capacity of dolphin musculature for observed swimming speeds. The paradox gave rise to a search for the means by which the necessary ten fold reduction in drag was induced, with focus variously on skin properties and body compliance. As detailed by Fish [17], the discrepancy was later found to be primarily the result of a fundamentally flawed calculation of available muscle power (combined with the fact that the animals in question were surfing the bow wave of the vessel from which the observations were made!) Regardless of the banality of the solution, however, the excitement helped to spur research into swimming animals and the promise of biorobotics.

The hydrodynamics of the side-to-side caudal fin motion of BCF swimming lends itself especially well to assumptions of planar flow about a foil chord section, simplifying computation, visualization and experimentation. Freymuth [22], [23] provided influential observations of the planar wake structure about plunging and pitching foils. Using smoke visualization in air, he identified the reverse von Karman street 
structure of the propulsive wake at high Reynolds number, with the sense of the vorticity contrasting it to the drag wake produced by the well known von Karman street behind a bluff body in steady flow. An enormous number of studies, too many do justice to here, have investigated propulsive wake structures in two dimensional and quasi-two dimensional foils, and the reader is encouraged to see Triantafyllou et. al. [65] for a review. Work at the MIT Towtank includes that of Gopalkrishnan [24] and Anderson [2] who categorized heaving and pitching foil wake structure by number and placement of vortices, showing a strong dependence of wake structural features on Strouhal number and angle of attack for sinusoidal kinematics. Much of this work follows from Triantafyllou et. al. [62], who related efficient thrust production to Strouhal number, and showed that animals across species and scales tend to operate within a narrow band of Strouhal numbers believed to be highly efficient for steady swimming. With the RoboTuna, a towed flexible bodied mimic of the BCF style swimming of the Giant Danio, researchers at MIT produced one of the most well known examples of a biomimetic system. Designed to study fish swimming in a repeatable way, divorced from conflicting biological imperatives of real animals, the Robotuna provided a platform for measurement of thrust production and efficiency, along with foil interaction with body vorticity, as documented in Triantafyllou and Triantafyllou [64].

Quantification of thrust production and efficiency for rigid foils, as a function of Strouhal number and maximum nominal angle of attack of the foil with respect to the flow, continued at MIT with work by Read [55] and Haugsdal [27]. A strong dependence of efficiency on foil chordwise compliance was shown by Prempraneerach [54], with increased efficiency strongly associated with reductions in maximum lift forces during the period of highest heave velocity (and hence large reductions in power output), which were accompanied by much lower drops in thrust. These results mesh with the hypothesis by Fish et. al. in [14] that passive cambering of caudal flukes plays a significant roll in oscillatory thrust production. Advancing past the use of simple harmonic kinematics, Hover et. al. [30] further demonstrated the utility of calculations of nominal angle of attack, even when disregarding induced flow on 
the foil, by demonstrating that modifying the kinematics of the heaving and pitching foil to more closely approximate a sinusoidal angle of attack results in increased efficiency and thrust production. The gains are particularly large where Strouhal number is large and maximum angle of attack low; the nominal angle of attack profile is especially degenerate here for the sinusoidal kinematics, with two maxima (or two minima) in the angle of attack profile for each half stroke.

A number of controlled systems, both free swimming and constrained have been constructed to test the control of systems using caudal fin style propulsion. Heading control is accomplished with strategies ranging from geometric control (Morgansen [50]), to sliding mode control (Saimek [56]), hybrid control, proportionalintegral-derivative (PID) control (Anderson [3]), and triggered discrete feedforward behaviors (Liu [44]). The model based control algorithms are typically based around quasi-steady hydrodynamic models such as the one presented in Mason [48], restricting accurate application to relative small perturbations about modeled trajectories. Pitch, depth and roll are either uncontrolled in all of these systems, or actuated using conventional control planes. [3] describes the Vorticity Control Unmanned Underwater Vehicle (VCUUV) developed by Anderson et. al., a free-swimming caudal fin propelled vehicle with onboard navigation and control, along with energy storage to allow for completely autonomous operation. The VCUUV achieves average turning rates several times higher than those of generic thruster/control plane maneuvering UUVs as modeled by the authors $\left(32^{\circ} / s\right.$ vs. $4^{\circ} / s$.) The VCUUV demonstrates both the promise and the complexity of propulsion premised on body flexibility, with the rear third of the vehicle flexing to provide the required high amplitude caudal fin motion.

Attempts to replicate the rapid start, as opposed to steady swimming, performance of BCF transient swimmers (fish using large body and caudal fin deflections to generate fast turning starts) include robotic systems using feedforward kinematics as in Liu [31] , and flexible, spring-loaded fish shaped bodies with varied tail shapes in Watts [68]. BCF transient turning by definition requires near full body flexibility, with extremely large accelerations of the full body; actuator power output limitations 
may account for the relative lack of success for fully integrated systems.

\section{MPF Swimming}

Another strand of biorobotic research, investigating the use of MPF swimming, has arisen concurrent with increasingly sophisticated understanding of the kinematics and functions of pectoral fin motion in both fish and mammals. Pectoral fin kinematics, and the associated fluid dynamics, are inherently three dimensional in a manner which does not lend itself to sort of planar flow visualization or quasi two dimensional computational techniques that illuminate caudal fin propulsion. Just as for BCF swimming, both bench mounted and free swimming systems have been built for the study of pectoral fin style actuation, with varying complexity in terms of both actuator and system design. Kato provides a recent review of MPF style actuation for marine vehicles in [35], noting that pectoral appendages are used in both drag based and lift based swimming styles. The basic thrust mechanism for lift based swimming is identified through laser flow visualization techniques by Drucker [10] as an unfolding series of connecting vortex ring which generate a jet opposite the direction of animal travel, and which can be reoriented to provide maneuvering forces.

The active conformability of fish pectoral fins currently far exceeds anything yet developed in a lab, however, and the magnitude of the contribution to maneuvering performance of this conformability, both of the individual fin rays and of the base attachment to the body, is an open question. Tangorra [60] has demonstrated the effect of actuating the dominant shape modes identified in bluegill sunfish by Lauder [41] in a flume mounted multi-rayed conformable fin, demonstrating an innovative nonlinear actuator mechanism which seeks to mimic the base mounted ray curving actuation

of many fish fin rays. Kato [36], [34] details perhaps the most complex pectoral fin deployed on a free swimming vehicle, with an actuator capable of moving a low aspect ratio foil (span and chord approximately equal) in three degrees of freedom independently. With a range of motion modeled loosely on the pectoral fin of the sea bass, a fuzzy-logic style controller was proposed and demonstrated for control of the vehicle in variety of maneuvering tasks including straightline swimming and motion tracking 
along underwater structures.. The complexity of the device may hinder the available power output, however.

Kemp [37] focused on the use of foil actuation that was three dimensional in the sense that the foil was attached to the body at only one end, but which was actuated on a single degree of freedom, twisting about the spanwise axis. With an emphasis on tuning of the foil geometry and compliance to the drive motors, the fins were successful in vectoring thrust within the plane perpendicular to the foil axis. The actuators were used both to provide enhanced low speed maneuvering capabilities as add on modules to vehicles with existing conventional thrusters [59] and as the sole means of propulsion for remotely operated [38] and autonomous vehicles [39]. The system suffers from low hydrodynamic efficiency $(<15 \%)$, however, and the inability to generate large foil velocities relative to quiescent flow limits actuator authority.

The 2DOF motion of rolling and twisting foils is an effective compromise between the complexity of the 3DOF motion of Kato, and the 1DOF motion of Kemp. The ability of rolling and twisting style rigid foils to generate thrust and maneuvering forces was demonstrated in towed foil experiments in Martin [47], helping to precipitate the work in this thesis by demonstrating extremely high maneuvering forces at speed through simple parameters changes in harmonic foil kinematics. Further experimental works by McCletchie [49] and Techet [61] with an apparatus modeled in part on the actuator described in this thesis, although more extensively instrumented, have found hydrodynamic efficiencies for rolling and twisting foils in excess of $75 \%$ with steady incoming flow, comparable to the $70 \%$ efficiency reported by Haugsdal [27] for heaving and pitching foils.

In recent work at the Naval Underwater Warfare Center, Beal [5] tested thrust production with a single foil in quiescent water to mimic thrust vectoring during vehicle hovering. The resulting mapping from foil kinematics to foil force was used to develop a controller for a fully actuated hovering vehicle with six rigid rolling and twisting foils. The vehicle consists of a single cylinder with three foils attached to each end cap, with the foils in their mean position extending straight out from the central vehicle axis spaced $120^{\circ}$ apart from one another. The foils can be operated 
in a synchronized "counter-rotating" mode for smooth surge actuation without unwanted attitude oscillation, or with varying frequency and phase depending on control algorithm demands.

Engineers should not feel constrained to produce vehicles that fall strictly within one category or another. Webb [70] posits that the inherent tradeoffs involved in design for different specialities and ecological niches will require that superior performance in one area will naturally be associated with degraded performance in other areas. In a review of fish functional design and perfomance, Blake [7] suggests that engineers taking inspiration from biology would do well to take this to heart. Indeed, Fish et. al [12] suggest that a truly versatile AUV would need to incorporate multiple fins with different sizes, shapes, and actuator capabilities.

\section{Non-piscine Inspiration}

Detailed study of body kinematics and hydrodynamics in controlled environments is a much more manageable proposition for small fish than for larger marine animals. However, while properly formulated parameters allow fundamental hydrodynamic mechanisms identified with oscillating foils to be scaled up, the same cannot necessarily be said for the structure and kinematics of actuators. Scaling up mechanisms for curving ray style fins is likely to be problematic, for example, as noted within Bandyopadyhay [4]. As noted in an excellent review of control surfaces in non piscine swimmers by Fish [16], paired foils/wings are typically less conformable, with high aspect ratios, particularly for those animals evolved for high speed swimming and maneuvering. While Fish highlights the extensive literature into the maneuvering capabilities of non-piscine swimmers, studies of wing and foil kinematics for swimming birds and large animals have generally been limited to beat frequency and body accelerations during steady swimming and the different phases of diving events, as in Lovvorn [46], with puffins, and Hays [28] with foraging sea turtles. Wyneken [75] and Davenport [33] have undertaken video study of foil kimematics for steady swimming in hatchling and juvenile turtles, but not comparable study exists for adults, and the author is not aware of any previous attempt to characterize adult turtle maneuvering 
foil kinematics.

\subsection{Chapter Preview}

Chapter 2 focuses on the design and baseline swimming capabilities of Finnegan. The design of the actuators is detailed, along with the sensing package, navigation algorithm, vehicle layout, and software approach. Experimental results for a single actuator are presented, with a method for extrapolating vehicle performance from the resulting foil characterization. Finally, simple harmonic foil kinematics are used to demonstrate the ability of the vehicle to independently translate in surge, sway, heave, and yaw.

Chapter 3 details the maneuvering performance of the vehicle using a control algorithm which perturbs the parameters of the harmonic kinematics, demonstrating turning performance that dramatically improves on the reported performance of existing UUVs. The maneuvering capabilities are dependent on large angle maneuvers such as banked and twisting turns, which are facilitated by the use of an attitude unstable trim configuration, and of the modified Rodrigues parameters as the underlying attitude description.

With Chapter 4, the focus of the work shifts to the direct use of biomimetic examples in vehicle maneuvering. In this chapter, the foil kinematics of "Myrtle", a Green sea turtle (Chelonia Mydas ) at the New England Aquarium, are presented for a number of basic maneuvers, including steady forward swimming, level turning and rapid pitching. While harmonic motion is a passable general approximation of marine animal foil use, it is clearly suboptimal for large angle maneuvers with time scales on the order of the foil oscillation period. Video capture from multiple angles was used to determine limb stroke plane with respect to the body, beat frequency, amplitude, and interlimb phasing, with the goal of identifying limb kinematics during useful maneuvers that could then be adapted for the vehicle.

In Chapter 5, the observations of the maneuvering turtle are used to generate feed-forward foil kinematics to actuate level turning in the vehicle. By modifying foil 
kinematics, the benefits of increasing foil roll range can be directly measured in terms of improved turning performance. Furthermore, the utility of viewing foil kinematics through the lens of nominal angle of attack is demonstrated by improvement of turning performance through approximation of a sinusoidal angle of attack on a foil using a lift based thrusting stroke.

Chapter 6 presents experiments with a single towed foil which demonstrate the role of inline foil motion in thrust production. Inline foil motion ameliorates the thrust penalty for a foil generating thrust during just the downstroke, as opposed to a foil generating thrust during both the downstroke and the upstroke. The results can be placed within the context of vehicle vs. turtle turning performance, where the inability of the vehicle to actuate inline foil motion is the primary difference between vehicle foil and turtle limb kinematics 


\section{Chapter 2}

\section{Vehicle Design and Open Loop Performance}

\subsection{Introduction}

In this chapter, the design of the vehicle is laid out, along with details of the foil actuator mechanism, of the sensing payload and navigation capabilities, and of the software and communications architecture. The basic harmonic foil kinematics which are used for the generation of thrust and lift in both open loop and closed loop vehicle operation are presented, as are the results from parametric studies of force production using foils intended for installation on the vehicle. Finally, the swimming performance of the vehicle without feedback control is given for for surge, sway, heave and yaw, along with an approach for estimating basic vehicle swimming capabilities for a given foil actuator set.

\subsection{Background: Biomimetic Vehicles in the Lit- erature}

Finnegan was intended to distinguish itself from existing free swimming biomimetic systems in that the biomimetic actuators provide the vehicle with both agility and 
maneuverability. Agility can be narrowly defined as the ability to change heading with no forward speed, but we advance a broader definition, as the ability to negotiate complex environments at low speed, which requires not only rapid heading change, but also independent actuation in surge, sway and heave. In this chapter, we document vehicle agility and the kinematics used to independently achieve 4DOF control at low speed, as well as the capability to reach maneuvering speeds in excess of $0.5 \mathrm{BL} / \mathrm{s}$. (Maneuvering at speed is addressed along with feedback control of vehicle heading and attitude in Chapter 3, where it is explored using turning radius as a function of speed as a metric.)

The Vortical Control Unmanned Underwater Vehicle described in [2] uses bodycaudal fin $(\mathrm{BCF})$ swimming to achieve speeds as high as $0.5 \mathrm{BL} / \mathrm{s}$, and demonstrates maneuverability far in excess of the typical streamlined UUV with turning rates as high as $30^{\circ} / \mathrm{s}$ using non-zero mean deflection of the caudal fin during harmonic oscillation of the vehicle tail to actuate yaw. The heading change is accomplished without appreciable loss of forward speed. However, the VCUUV steering is intimately tied to the forward propulsion, and it has no heading control without headway.

The Nektor actuator described in [38] can be used to provide vehicle agility through the capacity to vector forces within the the plane perpendicular to the foil axis with no incoming flow. However, while the actuators can be relatively easily deployed on a conventionally driven AUV to add low speed maneuvering capability, as in [59], the single degree of freedom (twisting only) actuation necessarily results in an inability to either generate large relative velocities between foil and flow with no flow speed, or to provide thrust given large relative flow velocity with respect to the vehicle mount point. These restrictions limit the magnitude of actuation during hovering, and reduce the top speed available to a vehicle powered solely by the foil, as is the case in [39].

In recent work at the Naval Underwater Warfare Center, Beal [5] tested thrust production with a single foil in quiescent water to mimic thrust vectoring during vehicle hovering. The resulting mapping from foil kinematics to foil force was used to develop a controller for a fully actuated hovering vehicle with six rigid rolling and 
twisting foils. The vehicle consists of a single cylinder with three foils attached to each end cap, with the foils in their mean position extending straight out from the central vehicle axis, spaced $120^{\circ}$ apart from one another. While the most similar to Finnegan in terms of the actuator mechanism and layout, the NUWC vehicle has so far only been tested in hovering tasks.

\subsection{Vehicle Design Details}

The vehicle performance specifications were formulated around a goal of providing both agility and maneuverability. Those specifications included a maximum velocity $O(B L / s)$, the ability to independently control six degrees of freedom, and better maneuvering capabilities at speed than existing autonomous underwater vehicles.

The functional requirements included the following:

- Foil based actuation - the primary goal of the program was to investigate the use of foils for propulsion and maneuvering.

- Scalability and flexibility - freedom to adjust foil actuator numbers, positions and orientations to allow insights into the tradeoffs between different vehicle layouts and the corresponding control strategies.

- Size constraints - $2 \mathrm{~m} \times 0.5 \mathrm{~m} \times 0.5 \mathrm{~m}$ maximum dimensions to make deployment problems in the MIT Towing Tank, swimming pools and the Charles River tractable.

- Autonomous operation - pre-programmed mission following, independent error handing, onboard power source, and data storage capability.

- Shallow $(10 \mathrm{~m})$, confined water operation - a greater depth rating adds cost and complexity with no corresponding increase in experimental functionality.

- Inertial navigation - minimal long term tracking accuracy required for short, supervised missions focused on local control issues. 


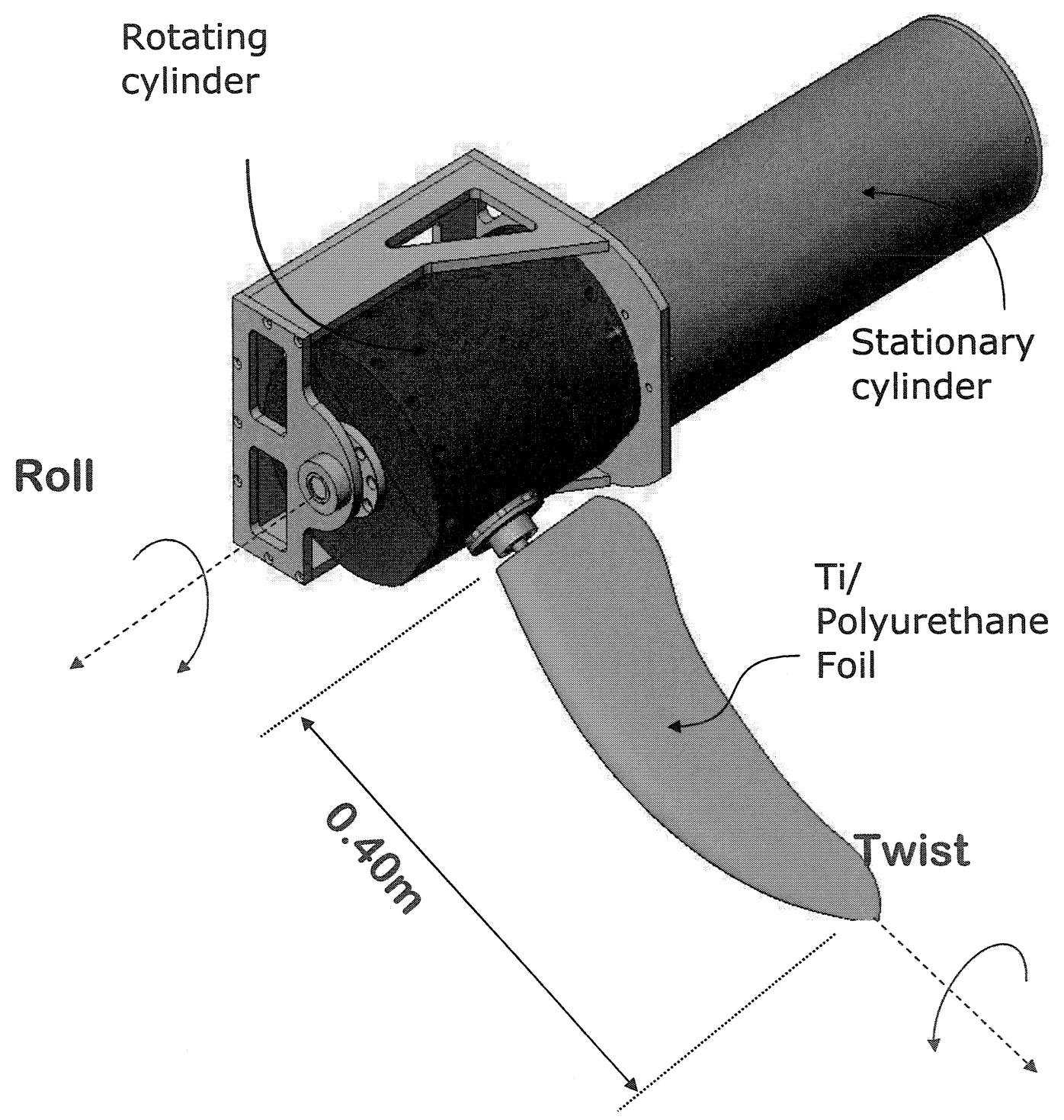

Figure 2-1: Dual Housing Actuator Design. The stationary cylinder contains a DC brush motor driving the rotating cylinder about the "roll" axis. The rotating cylinder contains a smaller motor which drives the "twist" axis.

\subsubsection{Foil Actuators}


The design effort for the vehicle was initially concentrated on the distinguishing characteristic of the vehicle, the foil actuation. A key requirement for the vehicle was scalability and flexibility in terms of the number of foils, as well as their positions and orientations. To meet the flexibility requirement, each foil actuator was conceived as part of a waterproof module that could be mounted anywhere on the vehicle frame, and be operated independently from the other foils. To allow for scalability, the modules are designed such that as more modules are added to the vehicle, there is no complexity added to the power and communication circuits.

A single foil module contains all the components necessary to add another foil to the vehicle. Each module contains a $196 \mathrm{~W}$ and a $15 \mathrm{~W}$ DC brush motor with optical encoders (Litton-Polyscientific, Blacksburg, VA) which actuate foil roll and twist, respectively. The corresponding motor control circuit is also housed in the module, with an Ethernet enabled 2-axis motion control card, and two PWM amplifiers. The addition of a new module entails only two connections: an Ethernet line to a central hub on the vehicle LAN, and a fused connection to the power bus. Since the hub acts in some sense like a bus connection, no additional wiring is required.

There were two generations of foil actuator designs. In the first design, all components were placed in a single housing, as drawn in Figure 3-8. While the mechanical actuation and internal wiring was straightforward within the single housing, the sealing was complex. The dynamic seal between the foil and the housing limited the depth of operation, range of motion, and the fatigue lifetime. After construction of a prototype, a second iteration was proposed to increase the depth rating of the actuator and the range of roll motion of the foil. The first prototype was installed in the MIT Propeller Testing Tunnel for experiments as described below.

The second iteration of the design, which was installed on the vehicle, contains the same electrical components in two independently sealed cylindrical housings, as shown in Figure 2-1. One cylinder remains stationary with respect to the vehicle and the second, smaller cylinder rotates about its axis with respect to the larger. The use of two independently rotating housings simplified the sealing problem, which improved the robustness of the seals. In addition, the full range of motion on the roll 
axis was improved to $\pm 85^{\circ}$, while the twist motion remained completely unrestricted. The rotary shaft seals, one of which is required for each degree of freedom, are still the most depth vulnerable aspect of the design. By the nature of flapping foil actuation, the force acting on each shaft is almost entirely perpendicular to the shaft axis, resulting in shaft deflections that, without outsized shafts, are well outside standard tolerances for o-ring. The elimination of shaft seals in foil actuation may come with the use of skin-like flexible materials which can be bonded to the foil shaft as well as the body, completely eliminating the potential leak paths that currently exist between o-ring/shaft and o-ring/housing. This solution will likely be viable only if the foil can still be effective with a limited twist motion.

In order to maximize the reliability, and hence the usefulness of the vehicle as a platform for different research teams, an emphasis was placed on simplicity and robustness of the actuators. One result is that mass of the solid moving parts in the dual housing design is relatively high, increasing the energy wasted on overcoming the rotational inertia, and potentially decreasing the bandwidth of thrust vectoring. Future designs should be made substantially smaller and lighter. In addition, once minimizing module size becomes a priority, e.g. with the need for payload space and reduced power operation, the cost of more compact components should become justified.

\subsubsection{Power Supply}

The power system is run at $24 \mathrm{~V}$ DC for safety and convenience, supplied by a pair of lithium-ion secondary cells from Valence Technology connected in series. Battery management electronics supplied with the batteries themselves are required to maintain cell balance within and between the two batteries during both charging and discharging. Battery cutoff from all vehicle electronics, other than the battery management electronics, is accomplished by connecting and disconnecting the battery to a DC/DC converter. The voltage difference on the low-voltage side of the DC/DC converter signals the battery management electronics to close a heavy duty power relay, drawing power to close the relay from the low-voltage side of the DC/DC con- 
verter. Both the vehicle power switch and the $\mathrm{DC} / \mathrm{DC}$ converter must accomodate the current surge required to close the power relay (and the trickle current required to keep it closed)l the power switch is a Seaconn-Giannini manual underwater switch. Power to all vehicle components is shut down if the battery management software detects a battery error state or if the switch is opened.

\subsubsection{Processors and Inter-Processor Communication}

The central processor for the vehicle is an Octagon Systems Pentium III single-board computer running RedHat Linux v7.2, while each actuator module contains a Galil 1425 2-axis motion control processor.

Each of the separate housings that comprise the vehicle are connected to an Ethernet LAN with a star-shaped topology centered on a housing containing an Ethernet hub and both a wireless access point and a bulkhead connector for tethered communication. The appeal of Ethernet lies in both high communication rates and the ease with which new components can be connected. The Galil motion control cards were chosen in part for their compatibility with Ethernet communication, and power distribution is controlled entirely through commands to an embedded server with digital I/O capabilities (a Hello!Device 1100 from Sena Technologies.) One result of the system architecture is that any computer running a web browser, and the OEM supplied software for the motion control card, can route power to one or more foil modules and control the foil motion directly.

The traditional argument against using Ethernet in control applications is that it is not structured to deliver information at deterministic times, but this is not a concern here. The microsecond timing required for the foil to accurately follow a predetermined motion path is dealt with at the actuator level in the motion control card, which is directly connected to the motor encoders. The higher level commands from the central processor must update only on the order of a fraction of foil oscillation period. The extremely low probability of packet delays over $10 \mathrm{~ms}$ on a small, quiet local area network (LAN) is inconsequential in comparison to a minimum foil motion period on the order of $0.5-1.0 \mathrm{~s}$. 
One dedicated processor was used to add a joystick interface to the vehicle. The joystick interface for Finnegan is based around a pair of 3DOF joysticks with a 1DOF rocker, providing a base level of seven channels of analog input. The joysticks are mounted on an enclosure containing a Micro/sys Embedded Systems single board computer running RedHat Linux 6.2, which communicates with the vehicle over an Ethernet connection. In addition to the analog input channels dedicated to the joysticks and rocker, 25 channels of input are available for other hardware inputs as needed. The mapping between inputs and foil action can be defined through software. Control over foil action and vehicle state can thus be apportioned between automatic control and manual control as appropriate for the current activity, as detailed in Chapter 3.

\subsubsection{Navigation Sensors}

A Crossbow 6-axis accelerometer in a dedicated housing located as close as possible to the geometric center of the vehicle (nearly the center of gravity by symmetry) is used for attitude estimation, with linear acceleration and angular rates updated on individual analog output lines at over $200 \mathrm{~Hz}$, and recorded at up to $100 \mathrm{~Hz}$ using a 32-channel 14-bit A/D converter from Micro/Sys.

The same A/D converter is used to log output from four narrow beam altimeters from Tritech Systems, each with a 10m maximum range, two located fore and aft on the starboard side pointing in the sway direction, along with one oriented down and one oriented orwards on the vehicle. The starboard altimeters are triggered over a serial line to avoid interactions, while the other two are free-running. All four operate at a maximum rate of approximately $10 \mathrm{~Hz}$, with update rate decreasing as range increases.

A pressure sensor from ParoScientific is used for depth measurement with mm precision; calibrated pressure data is relayed to the central processor over an RS-232 link. A downward facing WorkHorse Navigator 1200 doppler-velocity logger from RD Instruments provides velocity measurements, also over an RS-232 link with an update rate of approximately $2 \mathrm{~Hz}$, with update rate again depending on range. 

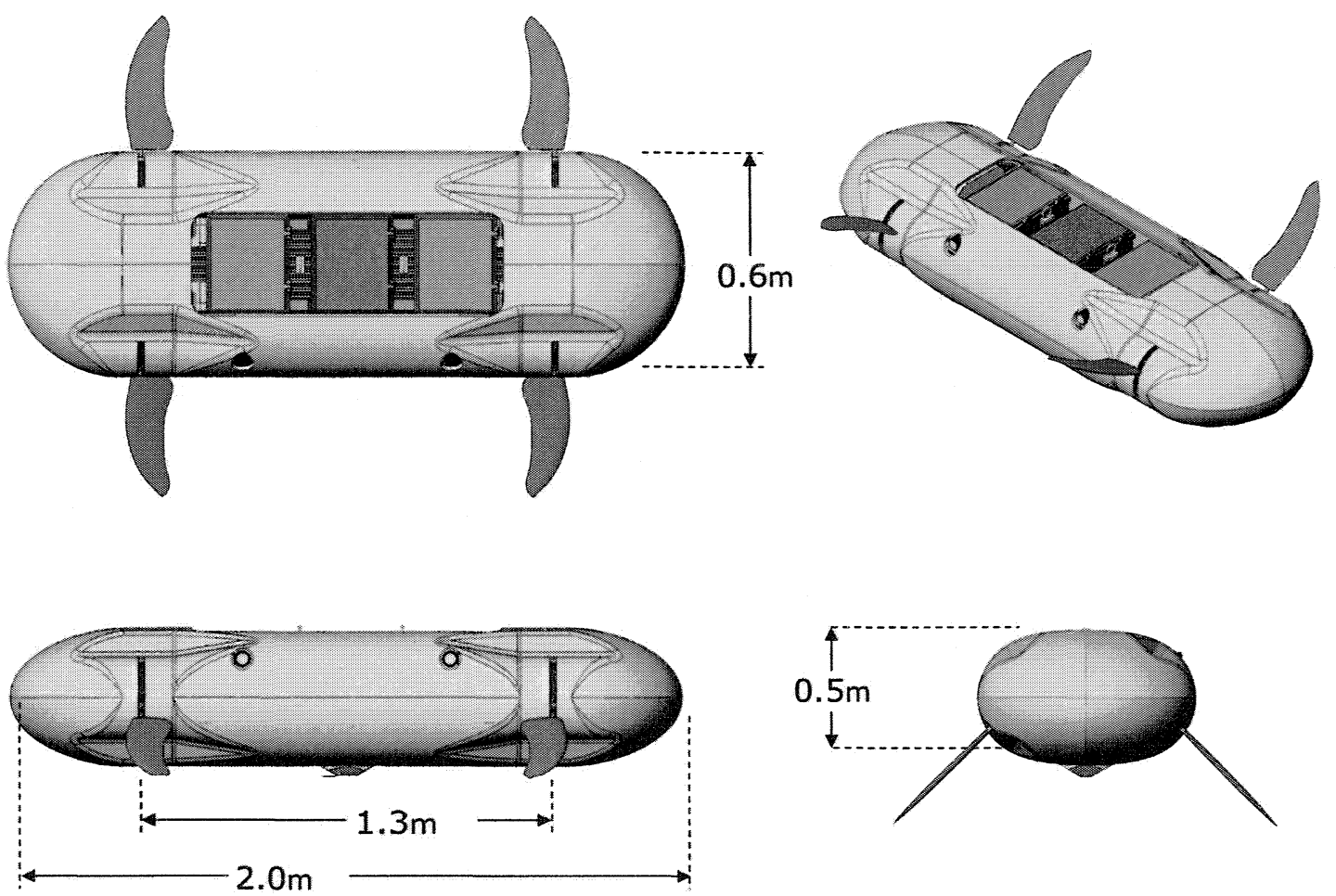

Figure 2-2: Vehicle dimensions with fairing installed. This trim/fairing configuration is used for all trials detailed in Chapter 2. The vehicle is stable in pitch and roll in this configuration, with the nose and tail of the fairing filled with shaped buoyancy foam. The fairing is flooded, with components individually waterproofed.

\subsubsection{Vehicle Configuration and Dimensions}

The two primary vehicle layouts envisioned for the vehicle involved four foils, placed so as to take advantage of port-starboard and top-bottom symmetry. The first configuration, which was ultimately adopted, consists of two pairs of foils placed portstarboard along the median line, at bow and stern, as drawn in Figure 2-2. (This configuration additionally results in fore-aft symmetry.) A second option, which was considered but never implemented, involved shifting one pair of the foils $90^{\circ}$ about the vehicle primary axis, so that they are oriented up-down, a configuration not unlike that adopted by the boxfish.

The primary advantage of maximizing symmetries was the resulting simplification of the control problem (see Chapter 3) and the increase in open loop stability of the vehicle, which is taken advantage of in the trials detailed below. The motion of 

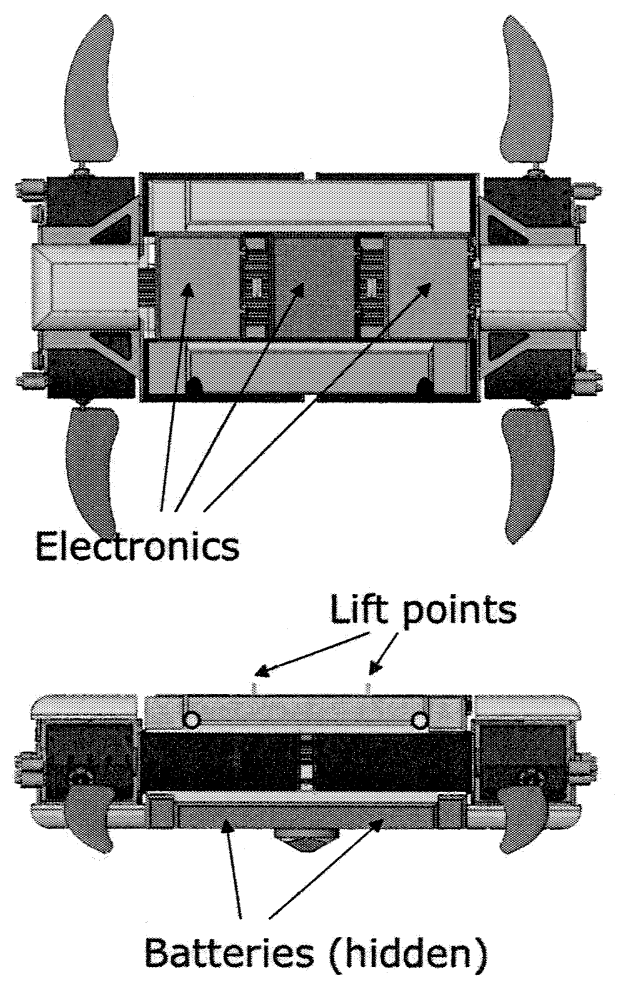

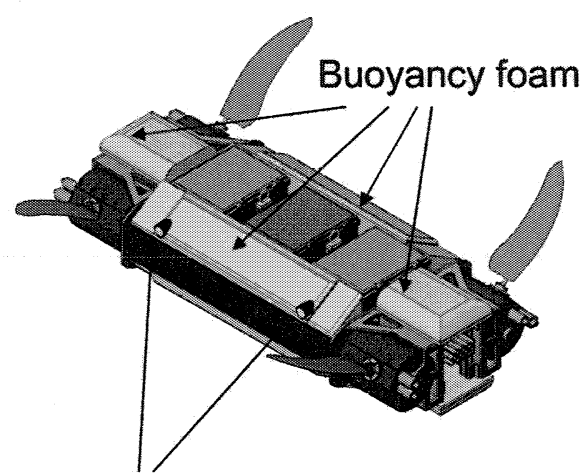

Sonar ranging

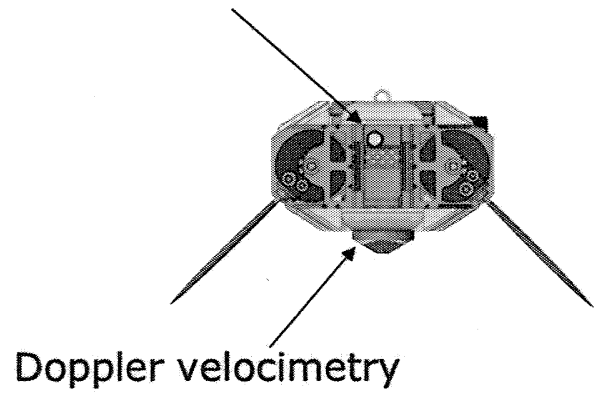

Figure 2-3: Vehicle sensor and construction detail. Vehicle is attitude unstable in this configuration, which is used for all trials detailed in Chapter 3.

the foils can be properly phased with respect to one another so as to cancel the unwanted cyclic forces that oscillating foils generally produce perpendicular to the desire impulse.

In the "sea turtle" arrangement, which was adopted for all experiments recorded in this thesis, the maximum vehicle dimensions using the streamlined fairing (and without foils) are $2 \mathrm{~m} \times 0.6 \mathrm{~m} \times 0.5 \mathrm{~m}$, while the foils protrude $0.4 \mathrm{~m}$ from each side, with $0.1 \mathrm{~m}$ average chord. When the vehicle was operated without the fairing (see Figure 2-3,) the vehicle dimensions are $1.3 \mathrm{~m} \times 0.5 \mathrm{~m} \times 0.6 \mathrm{~m}$. While less streamlined, the effective vehicle mass is also reduced: a substantial water mass is enclosed within the fairing when installed.

For the purpose of all testing with harmonically oscillating foils, the foil were limited to a $\pm 80^{\circ}$ range of motion in roll, and unrestricted motion in twist. (For the biomimetic maneuvers detailed in Chapter 5, the maximum negative excursion in roll 


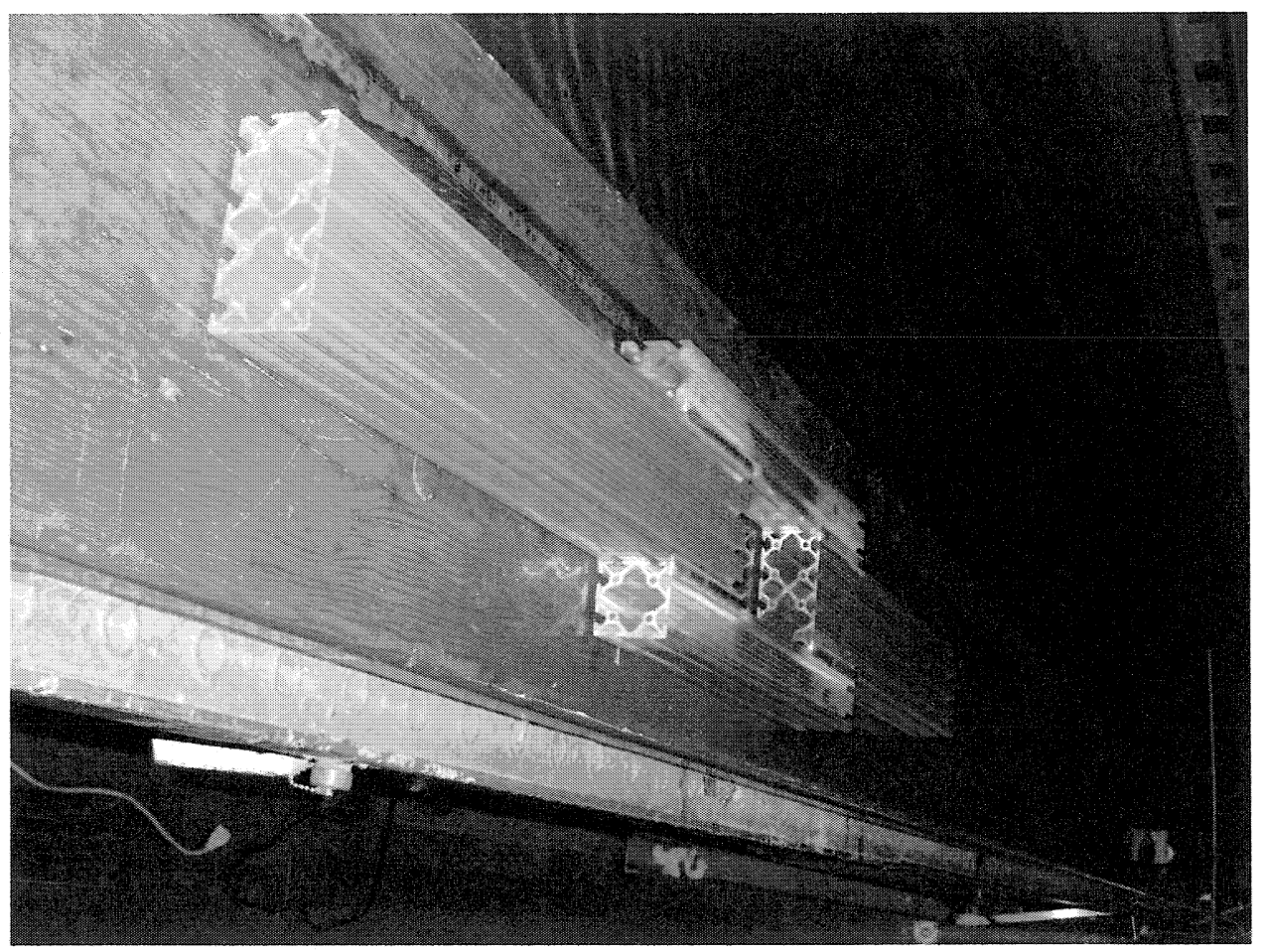

Figure 2-4: Detail of central aluminum spine.

was increased to $-120^{\circ}$, a design change directly inspired by the asymmetric range of motion in actual sea turtle forelimbs.)

As seen in Figure 2-3, the actuators were mounted to an erector set style aluminum spine welded from pieces with $5 \mathrm{~cm} \times 10 \mathrm{~cm}$ rectangular cross section (see Figure 2-4). All other vehicle components, including battery and electronics housings and foam for buoyancy were mounted directly to the same spine. Buoyancy billets of closed cell polyurethane foam were arranged around the components as shown in Figure 2-5.

\section{Turtle-like Morphology}

Finnegan is configured to use four identical foils, despite the lack of direct biological analogy (other than the now extinct Plesiosaur [51].) More typical of marine animals using high aspect ratio foils for propulsion is a single pair of foils forward of the vehicle center of gravity. Indeed, a vehicle with a single pair of oscillating foils forward, with a pair of control surfaces rather than a second pair of oscillating foils in the rear would accrue significant advantages in terms of cost, payload capacity, and reliability. With 


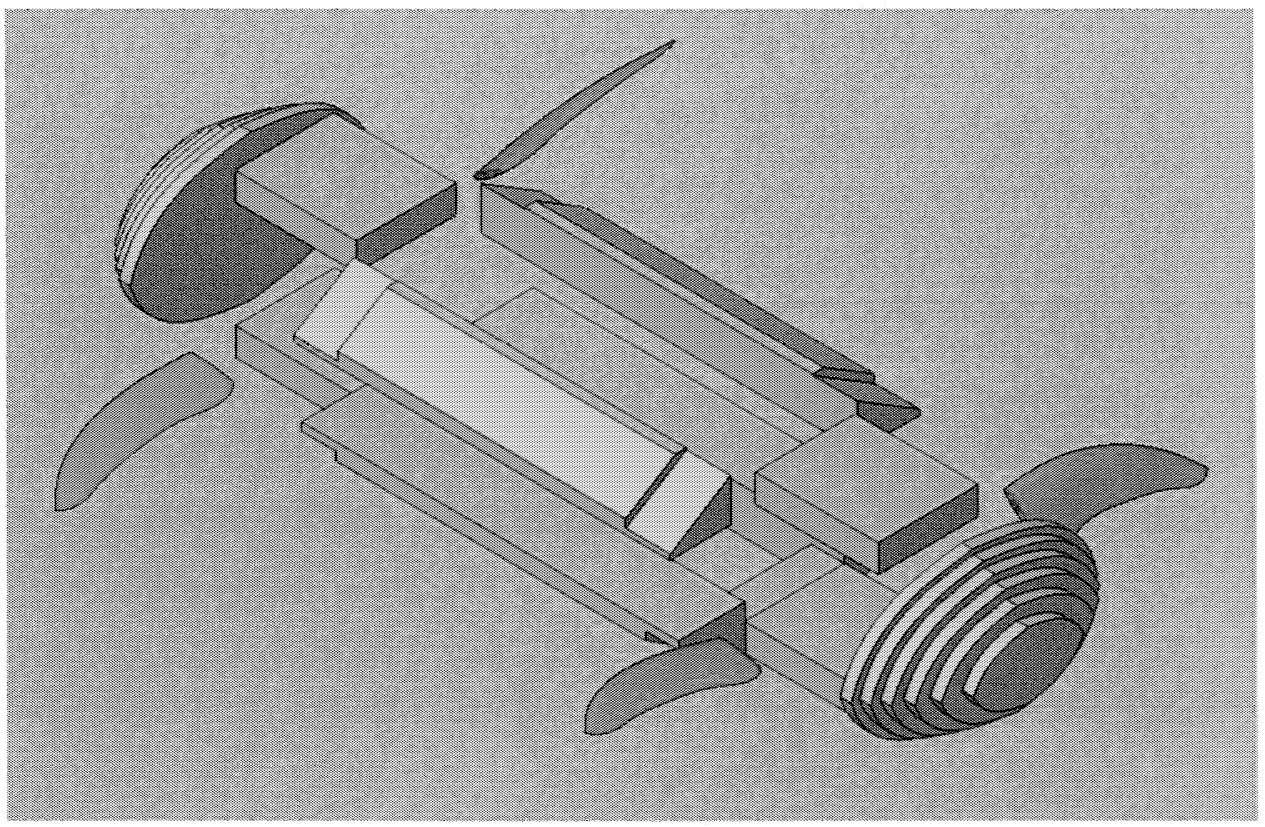

Figure 2-5: Detail of available buoyancy foam sections.

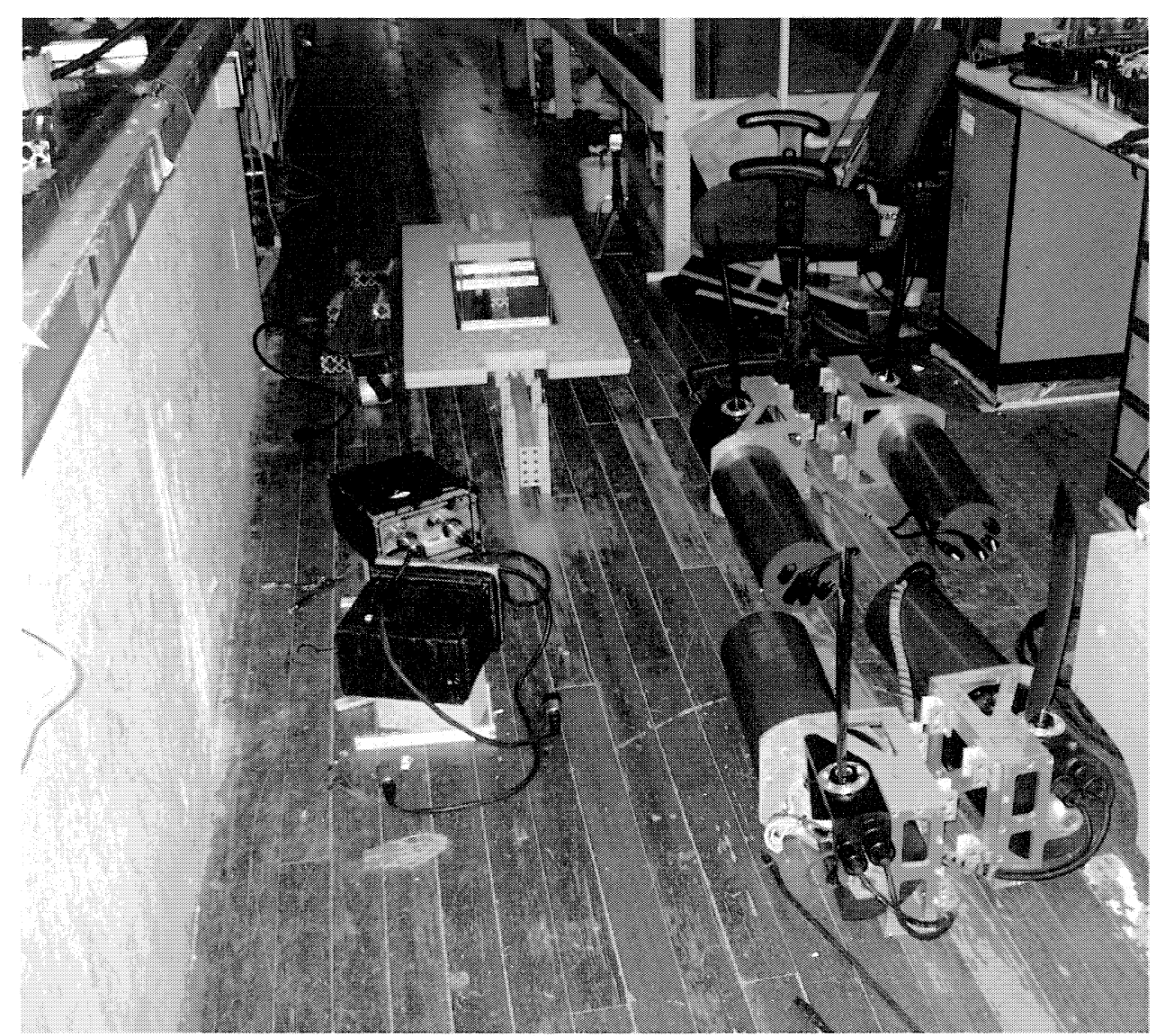

Figure 2-6: Vehicle components during assembly at the MIT Towing Tank. 
the current foil actuator design, reducing the number of oscillating actuators from four (two pairs, fore and aft) to two (one pair forward) would nearly double the sensor payload capacity or, alternatively, reduce vehicle total mass by approximately $30 \%$. As currently constructed, non-payload cost would be reduced by as much as $40 \%$. In addition, two rotary shaft seals with moving sealed surfaces would be eliminated, and the complexity of two of the remaining six rotary seals would be dramatically reduced, as they would only be required to withstand shaft deflections caused by use as conventional control surfaces.

In [45], Pell et.a al argue that the use of four foils on an oscillating foil vehicle provides no advantage for a rapidly cruising vehicle in terms of top speed or propulsive efficiency, due to the interaction between the trailing foil pair and the wake of the leading foil pair. Their analysis is limited to foils free to oscillate in twist only, however, meaning that there is no recourse to gross motion of the foil to position it with respect to the the oncoming wake. Indeed, anguilloform fish (Liao [42]) can utilize structured wakes with length scales comparable to the length scale of the actuation, such as might be produced by an upstream foil, in order to increase efficiency of the downstream foil. However, any attempt to take advantage of this effect clearly involves some flow sensing capability, as is the case for the wake energy extraction successfully demonstrated by Beal [6] with heaving and pitching foils.

Regardless of the effect of steady forward swimming, the reduction in complexity, increase in robustness, and decrease in cost and weight, would inevitably be accompanied by reduced functionality. A vehicle dropping from four foils to two would lose the ability to translate directly in heave and sway; whether or not this trade-off is favorable necessarily depends on the functional requirements of the vehicle. Breaking vehicle symmetry by removing an actuator pair may decrease the mechanical complexity of the system mechanically, but this will increase the control complexity, particularly at low speeds. The single pair of foils will be called upon to generate forward thrust and to control heading and attitude. The force vectoring abilities of the foils, which are capitalized on in maneuvering trials in Chapter 3, are described in a cycle to cycle averaging sense, a point which can be glossed over to some extent 
when differential actuation with symmetrically placed actuators is used; the oscillatory component of the force from each foil is balanced by the oscillatory component of the opposing foil. Without symmetry, however, balancing forces through the entire stroke would not be possible for all desired vehicle action.

In the final analysis, the added ability to perform the lateral and vertical translations, along with the simplification of vehicle control, were deemed to outweigh the disadvantages of added actuation. The use of four foils does not, however, prevent experimentation using just the forward foil pair; the results from the various adaptations of sea turtle limb kinematics tested in Chapter 5 make it clear that the heading change is possible with appropriate use of forelimbs only.

\subsection{Foil Kinematics}

Throughou this thesis, the large displacement flapping motion of the wing is referred to as the roll motion and the feathering of the wing is referred to as the twist motion. Refer to Figure 2-1 for schematic indicating rotation axis with respect to actuator. The basic motion of the foils, referred to throughout as "harmonic" or "simple harmonic", is sinusoidal in both roll $(\phi)$ and twist $(\theta)$.

The roll position of the foil is defined as,

$$
\phi(t)=\phi_{0} \sin (\omega t)+\phi_{\text {bias }}
$$

where $\phi_{0}$ is the roll amplitude in radians and $\omega$ is the frequency of the foil motion in radians per second. $\phi_{\text {bias }}$ is a static roll bias used to change the mean roll position of the foil. When multiple foils are in use on a vehicle, there may also be a phase difference between foil roll motions.

The twist position of the foil is defined as,

$$
\theta(t)=\theta_{0} \sin (\omega t+\psi)+\theta_{\text {bias }}
$$

where $\theta_{0}$ is the twist amplitude in radians and $\psi$ is the phase angle between twist 
and roll in radians. $\theta_{\text {bias }}$ is a static twist bias used for maneuvering. The phase angle, $\psi$, for all experiments described herein, is $\frac{\pi}{2}$ and we can therefore write $\theta(t)$ as,

$$
\theta(t)=\theta_{0} \cos (\omega t)+\theta_{\text {bias }}
$$

For heaving and twisting foils, the motion is non-dimensionalized using three parameters: Strouhal number $(S t)$, maximum angle of attack $\left(\alpha_{\max }\right)$, and heave amplitude to chord ratio. The corresponding parameters in rolling and twisting motion for a flapping foil are the $S t$ and $\alpha_{\max }$ as calculated at a location $70 \%$ of the distance from the root of the foil to the tip. The distance to this point from the axis of roll rotation is denoted by $r_{0.7}$. The ratio of the arc length at $r_{0.7}$ to the chord, denoted as $\frac{h_{0.7}}{c}$ replaces the heave amplitude to chord ratio.

Now for three dimensional kinematics we can express the angle of attack at $r_{0.7}$ as,

$$
\alpha(t)=-\arctan \left(\frac{\omega r_{0.7} \phi_{0} \cos (\omega t)}{U}\right)+\theta_{0} \cos (\omega t)+\theta_{\text {bias }}
$$

For three dimensional kinematics, the Strouhal number is defined,

$$
S t=\frac{2 r_{0.7} \phi_{0} f}{U}
$$

The Strouhal number can be thought of as a measure of the aggressiveness of the flapping motion with respect to the incoming flow speed. Maintaining the same $S t$ while increasing the flow speed requires an increase in flapping frequency, amplitude or both. The factor of two results in scaling as a function of approximate wake width, which emphasizes the relationship between $S t$ and vortex shedding patterns in the foil wake. 


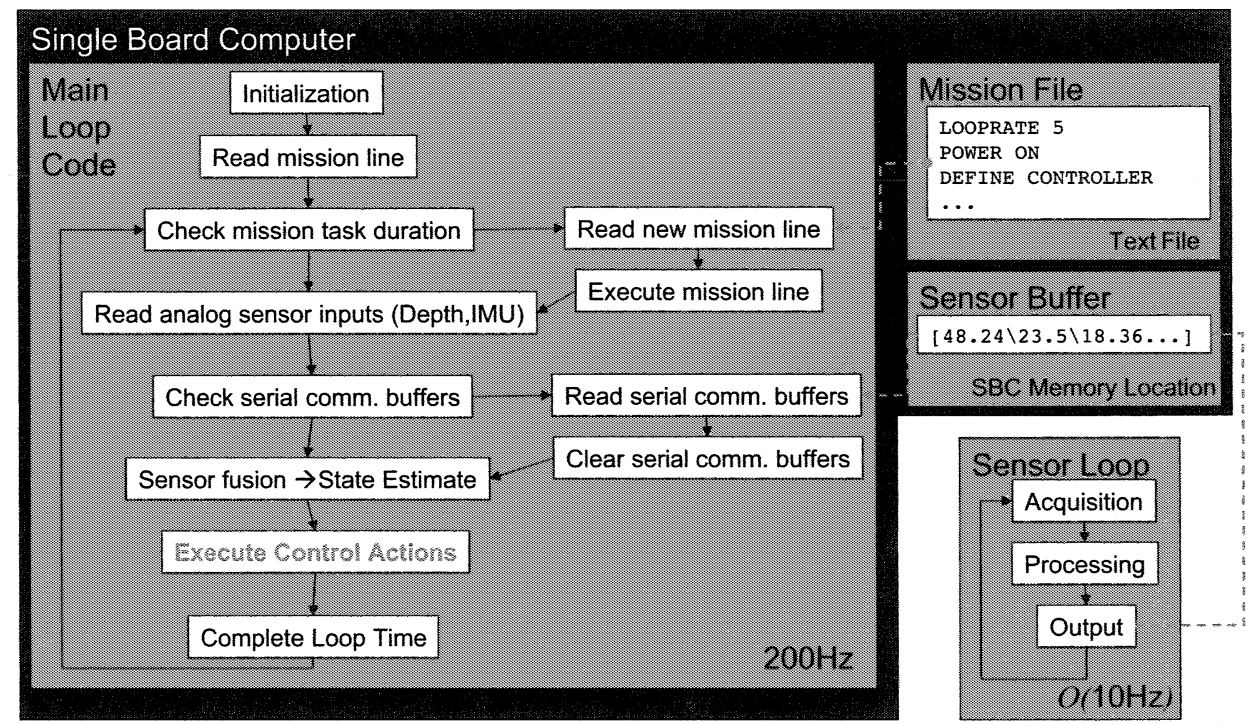

Figure 2-7: Block diagram illustrating flow of underlying operational code.

\subsection{Code Theory of Operation}

\section{Operating Modes}

The operator controls the vehicle with text commands through a windowed user interface on a remote terminal. Once the user interface is invoked, the operator can switch back and forth between "shell" mode and "mission" mode. In shell mode, the operator is able to initiate and test communications between subsystems, power the actuators on and off, send commands to the foil actuators individually or collectively, and view data files and mission scripts. Mission mode is entered by invoking a prewritten mission script. To exit back to shell, the operator either aborts the mission or waits for the script to finish executing.

\section{Initialization}

During mission mode, the vehicle operational code follows the flow diagram shown in Figure 2-7. When a mission is first invoked, an initialization sequence performs the 
following tasks:

- Start main operational loop timing process.

- Spawn multiple threads and memory buffers to handle low frequency serial communications with subsystems.

- Invoke execution of trajectory generation code loop on embedded motion control processors in foil actuators.

\section{Mission Timing}

After initialization, the main operational loop executes with a frequency that can be set within the mission script (the default loop rate is $10 \mathrm{~ms}$, or $100 \mathrm{~Hz}$.) Each execution of the loop begins with a test to see if all "script wait" commands have expired. Wait commands define mission timing by setting both fixed length and event driven waits. Event driven waits can set to expire depending on either vehicle state, or foil actuator state. A typical use of wait commands would be a timed wait for the vehicle to reach a steady speed with some foil kinematics, followed by an event driven wait during a turn which expires when some reference heading is reached. If communication with the remote terminal is maintained during the mission over a tether, the operator can force expiration of the current wait command manually

If all wait commands have expired, the next script line is read and acted upon. Aside from timing commands, script lines can be used to set reference heading, depth and attitude; select and activate vehicle control modes; set general execution parameters such as the main loop rate; and set foil actuation behavior.

\section{Data Acquisition}

As shown in the flow diagram in Figure 2-7 data is acquired with every loop execution from the inertial measurement unit (IMU) through analog data acquisition. A test is then performed to see if any of the (relatively slowly updating) instruments communicating through the various serial devices have posted new readings to the 
sensor memory buffers. The serial devices are monitored by blocking reads on the separate threads that were spawned during initialization. Data from the pressure sensor, acoustic doppler velocimeter, and two of the four altimeters is acquired in this manner.

Unsolicited messages from the embedded foil motion control processors containing position, velocity, and torque data are then handled if received over the Ethernet LAN, as are messages from the processor monitoring joystick position if joystick input is enabled.

Vehicle attitude and depth estimates are calculated with each loop, and this state and any available sensor data is written to a single formatted, time-stamped, line in an ASCII text file.

\section{Foil Control}

The last step before the main execution loop is repeated is to update the foil motion trajectories. The embedded foil motion control processors are initialized to follow trajectories which are updated every $64 \mathrm{~ms}(15.6 \mathrm{~Hz})$. Two trajectory update modes are used: internal and external. The internal trajectory update mode is used to generate sinusoidal oscillations; code on the motion control processor generates the next trajectory update based on parameters that are sent from the central processor. The parameters consist of frequency, amplitude, bias and phase for each axis. Smooth transitions between oscillations with different parameters are calculated internally, using an algorithm chosen from a small set of possibilities; the selection of the transition algorithm is dependent on the nature of the change in the parameters. In this thesis, the internal trajectory mode is used in one of two ways:

1. Foil oscillating parameters are updated in a predetermined pattern at timed intervals. The parameters are directly set in mission script lines which are interspersed with wait commands to establish the timing.

2. Foil oscillating parameters are updated $n$ times every cycle, as dictated by a feedback control algorithm, where $n=(1,2)$. Control gains and reference values 
- Sample Mission File

- LOOPRATE 5

- POWER ON

Timing control

- RECORD DAQ ON

- RECORD SERIAL ON

- I

- define controller start

- MODE 1

$\begin{array}{ll}\text { MODE } & 1 \\ \text { UPDATE } & 10\end{array}$

SWAY REF

DEPTH REF

YAW REF

YAW_GAIN

10
0
0

0

YAW

1050

START CONTROL

Closed loop control invocation and timing.

END CONTROL

I

CT A FR=1;AMX $=30 ; A M Y=45 ; B I A S X=0 ; B \mid A S Y=0 ; P H I Y=0 ; P H I X=0 ; C H A N G E=1 ;$

WAIT 10000

CT ADIRECT $=-1 ;$ CHANGE=1;

WAIT 10000

CT AMO;

RECORD DAQ OFF

RECORD SERIAL OFF

POWER OFF

Figure 2-8: Annotated example of mission script file.

are set within the mission script.

The first method is used for the open loop performance trials in this chapter, while the second method is used for the closed loop maneuvering trials in Chapter 3.

The external trajectory mode is used to drive the foils along arbitrary trajectories, with the trajectory updates coming directly from the central processor. In contrast to the internal trajectory generation mode, where the foils can operate indefinitely without commands from the central processor, the external trajectory mode requires that the desired foil positions be communicated from the central processor with every trajectory update, i.e. every $64 \mathrm{~ms}$. External trajectory mode is used for the feedforward biomimetic maneuvers tested in Chapter 5, with the desired foil trajectories written ahead of time to text files which are then read line by line during mission execution. 


\subsubsection{State Estimation}

Absolute position and absolute heading information was, in general, not available during experiments at the MIT alumni pool. ${ }^{1}$ This lack of absolute position information did not affect the results of the open loop velocity tests presented in this chapter, and in the following chapter, where maneuvering capabilities are tested, vehicle state estimation was performed using dead reckoning, relative to the initial condition at some starting point.

Two instruments were used to produce the dead reckoning estimates. Vehicle attitude was determined from a complementary filter fusing the accelerometer data and the rate gyro data, both updated at $>100 \mathrm{~Hz}$ from the Crossbow intertial measurement unit (IMU). The vehicle velocity estimate, to be combined with vehicle attitude for integration in dead reckoning, was developed from the RDI acoustic doppler velocity log (DVL.) The calibrated output from the DVL was transformed to reflect the orientation of the DVL on the vehicle, and the relative motion of the instrument with respect to the vehicle center of gravity due to angular rotation in pitch and roll. The correction for vehicle rotation was especially significant during the large angle maneuvers detailed in Chapter 3. The effect of yaw rotation was negligible, as the center of DVL transducer was coincident with the vehicle z-axis. Where long periods of DVL drop out $(>0.5 \mathrm{sec}$ ) were observed during large angle maneuvers or rapid acceleration, trials were considered to be invalid due to poor modeling of actuator and vehicle hydrodynamics.

The depth was established using the ParoScientific pressure sensor, based on the estimated vehicle attitude and the known position of the pressure sensor input port relative to the vehicle center of gravity. In the absence of new sensor readings, depth was calculated using dead reckoning from the last known depth in the same manner as the $\mathrm{X}$ and $\mathrm{Y}$ position.

\footnotetext{
${ }^{1}$ In certain limited cases not treated here, the vehicle position and heading within the pool was successfully estimated from the four altimeters.
} 


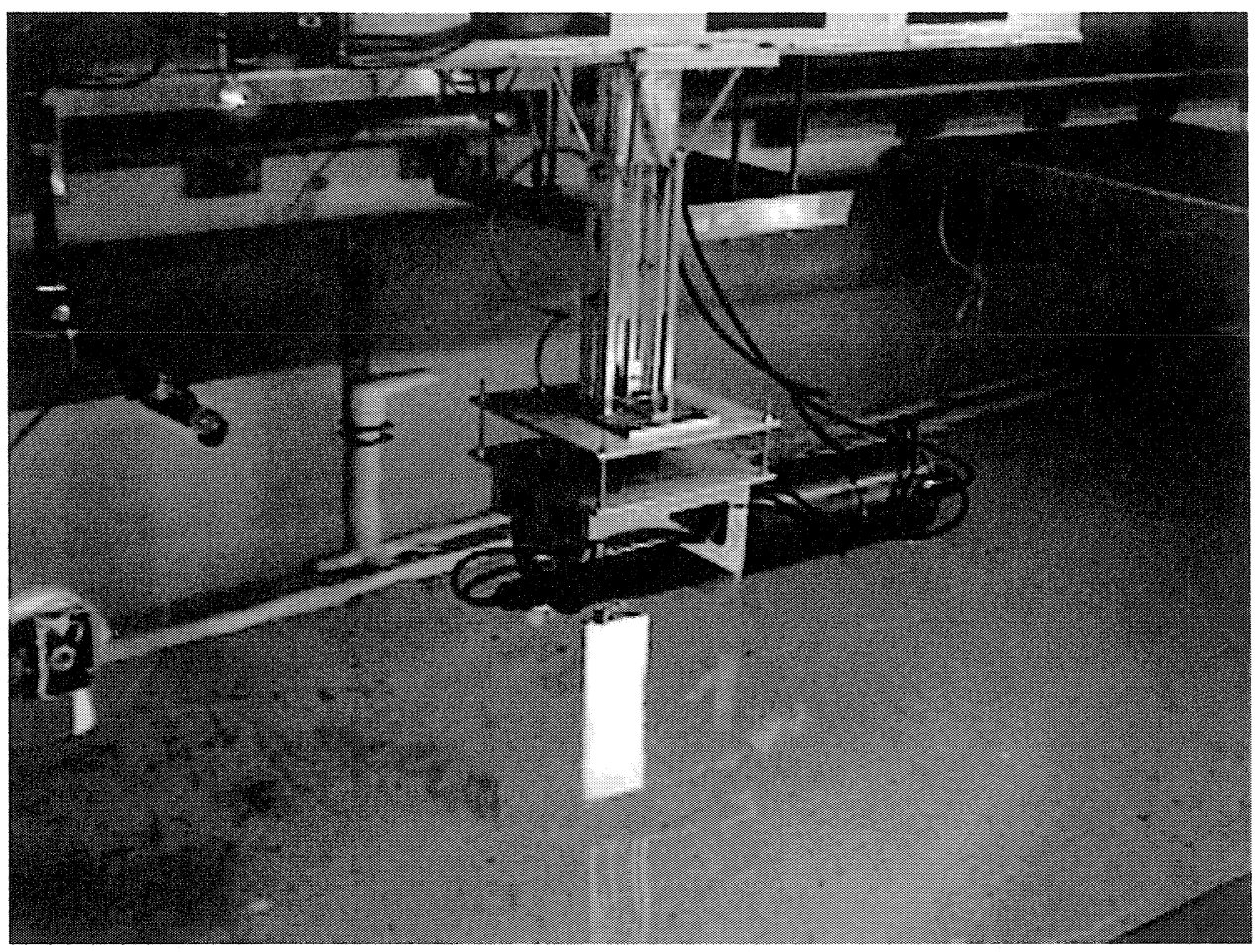

Figure 2-9: Towtank Actuator Test Apparatus

\subsection{Practical Design Tools for Foil Actuation}

Before multiple foil actuators were built and integrated into a free swimming vehicle, extensive testing was performed in the MIT Water Tunnel and MIT Towing Tank by Polidoro [53] to verify that the foils would be able to produce the desired thrust and survive extended operation.

Polidoro mounted one of the four actuators constructed for use on the vehicle to the MIT Towing Tank towing carriage below a two axis dynamometer with the foil submerged (Figure 2-9). Testing was performed across a 4-dimensional test matrix, varying $S t, \alpha_{\max }, \phi_{0}$ and foil aspect ratio. The details of the experimental apparatus and the complete experimental results can be found in [53]. Figure 2-10 plots mean thrust coefficient $C_{T}$ developed as a function of the non-dimensional parameters for operation with $\phi_{0}=60^{\circ}$, with a foil span of $40 \mathrm{~cm}$ and constant chord of $10 \mathrm{~cm}$. Non-dimensional lift and thrust coefficients $C_{L}$ and $C_{T}$ are calculated throughout as, 


$$
\begin{aligned}
C_{T} & =\frac{T}{\frac{1}{2} \rho U^{2} A_{\text {foil }}} \\
C_{L} & =\frac{L}{\frac{1}{2} \rho U^{2} A_{\text {foil }}}
\end{aligned}
$$

where $T$ and $L$ are the thrust and lift force, $\rho$ is the fluid density, $U$ is the flow velocity and $A_{\text {foil }}$ is the foil area.

The actuator thrust output range increases as the roll amplitude is increased, with mean thrust coefficients as high as 7 available to the actuator with $\phi_{0}=60^{\circ}$. The shape and height of the contours of $C_{T}$ with respect to $S t$ and $\alpha_{\max }$ are largely unchanged by $\phi_{0}$, however, with the increase in maximum available $C_{T}$ resulting from the higher $S t$ that can be attained when a larger stroke is used. All of the towed experiments were performed at a towing velocity of $0.5 \mathrm{~m} / \mathrm{s}$.

Plots of forces normalized by foil area and flow speed theoretically indicate how much force a foil of a given size can generate at any flow speed. However, there is an implicit assumption that the mechanism driving the foil is capable of delivering the power required regardless of the speed. In other words, while the fundamental physics underlying the efficiency and thrust development of flapping foils is illuminated by the normalization, the process hides the specific performance limits of the actuator under test. In reality, on a normalized force plot, the attainable region will shrink as the flow speed increases because of actuator limitations. To make useful predictions for real actuator performance, a number of assumptions need to be made:

- The distance, $r_{e f f}$, from the roll axis to the effective center of force on the foil is a function of Strouhal number and $\alpha_{\max }$.

- Maximum lift is experienced at the maximum roll angular velocity.

- Maximum roll motor torque coincides either with maximum lift or maximum foil acceleration.

- Added mass of water in rotation about the roll axis is much smaller than the rotational inertia of the moving parts of the actuator. 


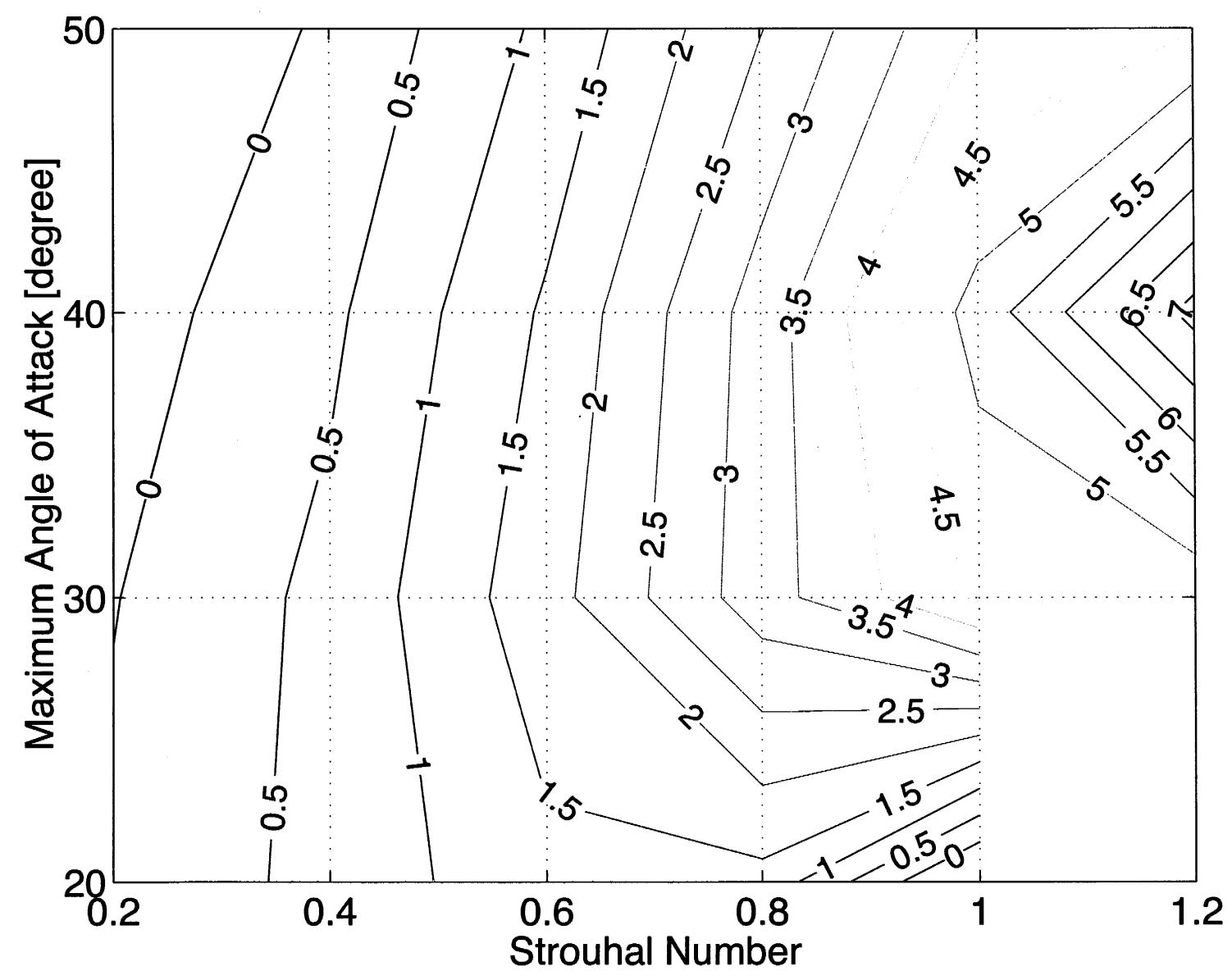

Figure 2-10: Contours of thrust coefficient, $C_{T}$, for a harmonically rolling and twisting rigid foil with roll amplitude $\phi_{0}=60^{\circ}$, resulting in $h_{0} / c=4.8$ at $r_{0.7}$. Foil span is $0.4 \mathrm{~m}$, with constant NACA- 012 cross section, chord length $0.1 \mathrm{~m}$. (Compare to vehicle foil, partially flexible with $0.4 \mathrm{~m}$ span, and maximum chord or $0.1 \mathrm{~m}$.) Data from experiments performed by Polidoro in [53] and previously published by Licht [43].

The motor torque requirement at the moment of maximum $C_{L}$ should scale with the lift force, $L$, when the Strouhal number, $\alpha_{\max }$ and $\theta_{\max }$ are unchanged,

$$
\tau_{\text {max }}=L_{\text {max }} r_{e f f}
$$

Hence, the roll motor torque requirement at the moment of maximum lift scales with $U^{2}$,

$$
L_{\text {max }}=\frac{1}{2} \rho U^{2} A_{\text {foil }} C_{L \max } \sim U^{2}
$$

By the assumptions above, the roll motor speed requirements peak at this moment 

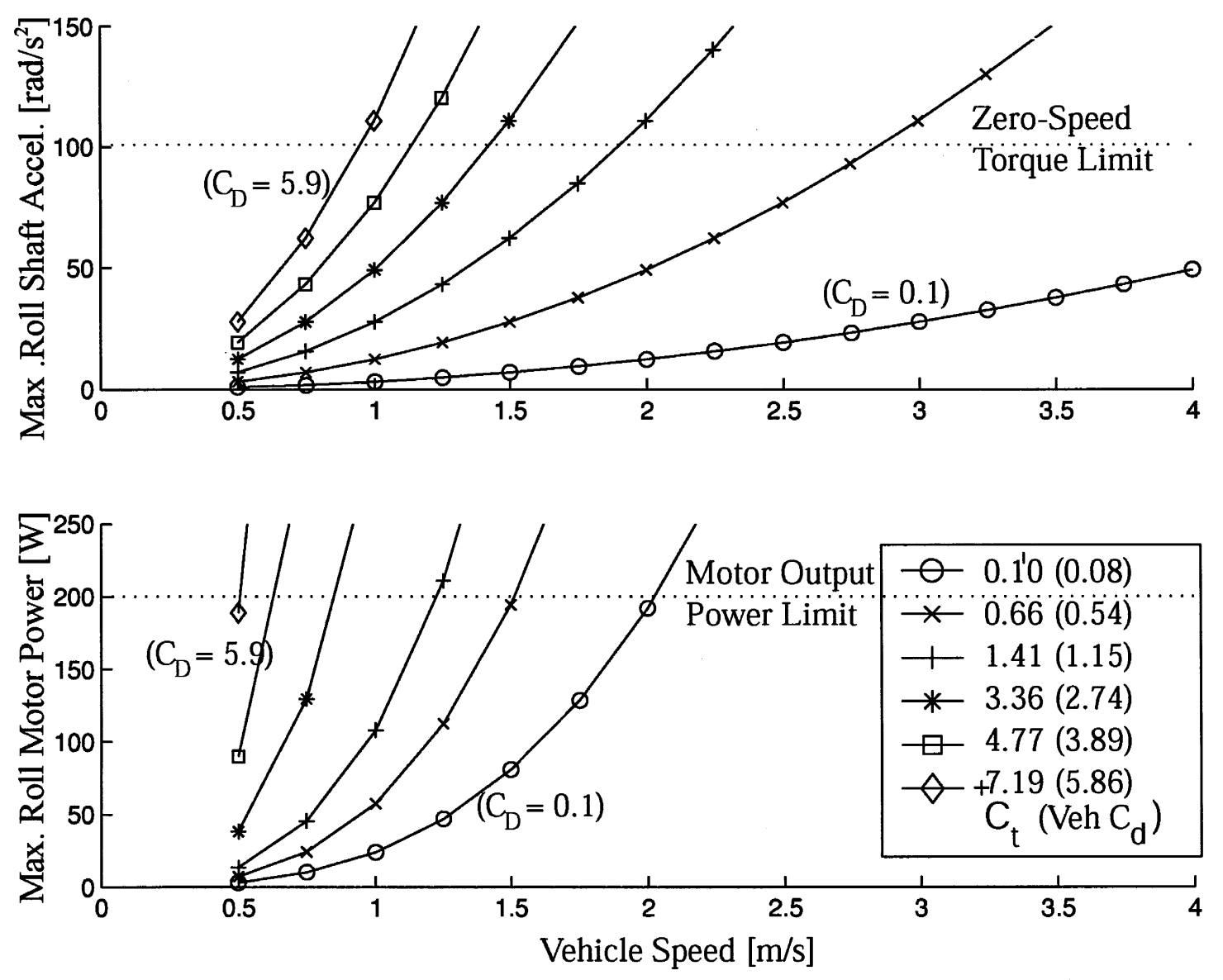

Figure 2-11: Estimation of Vehicle Speed Limits with Varying $C_{D}$ : Power and acceleration limits. Separate curves represent scenarios with different vehicle drag coefficients, $C_{D}$, and hence different foil thrust requirements. The speed at which the curves cross the constant angular acceleration and power output limits indicates an estimate of the vehicle top speed for the corresponding vehicle $C_{D}$. All curves are extrapolated from experimental data points in Polidoro [53], where vehicle actuators where tested at towed speed of $0.5 \mathrm{~m} / \mathrm{s}$.

as well,

$$
\begin{gathered}
\dot{\phi}=\phi_{0} \omega \cos \omega t \\
\dot{\phi}_{\text {max }}=\phi_{0} \omega
\end{gathered}
$$

From (2.5),

$$
\phi_{\circ} \omega=\frac{U \cdot S t}{r_{0.7} \pi} \sim U
$$

Hence shaft speed scales with flow velocity as well. Since output power for the 


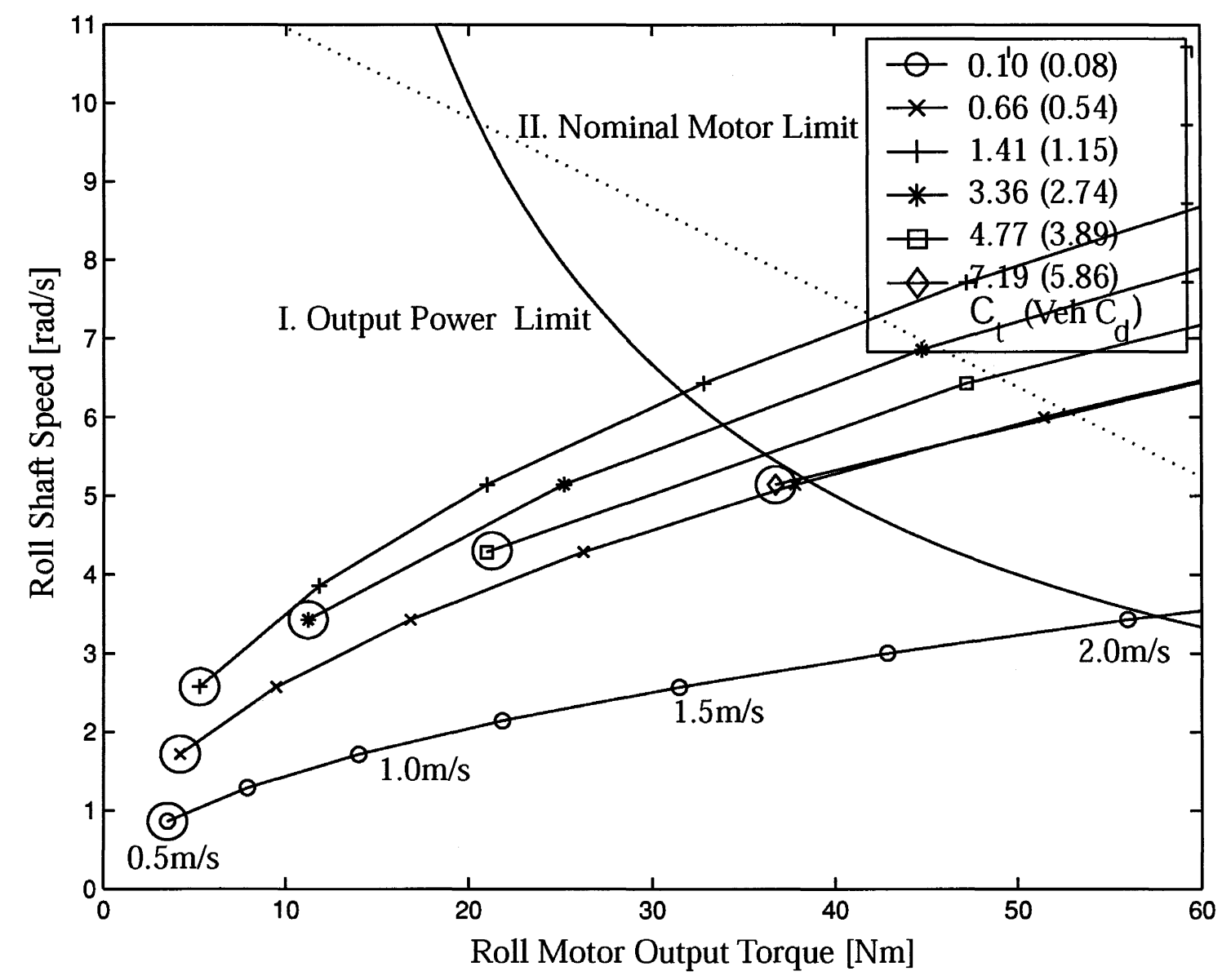

Figure 2-12: Estimation of Vehicle Speed Limits with Varying $C_{D}$ :Torque-speed requirements with flow speed increase. Curve I represents constant power output limits. Curve II represents nominal torque-speed curve.

motor is calculated as,

$$
P=\tau \omega \sim U^{3}
$$

it follows that power output required at the moment of maximum lift scales with the flow velocity cubed when $S t$ and $\alpha_{\max }$ are held constant.

The rotational inertia must be accounted for at the point of maximum foil roll acceleration, where,

$$
\begin{gathered}
\ddot{\phi}=-\phi_{\circ} \omega^{2} \sin \omega t \\
\left|\ddot{\phi}_{\text {max }}\right|=\phi_{\circ} \omega^{2}
\end{gathered}
$$


Ignoring the added mass of the fluid, which we do not know as a function of $S t$ and $\alpha_{\max }$, we find that the torque required to overcome the rotational inertia in the roll scales linearly with the roll amplitude and with the square of the frequency. The discussion of actuator limits here has focused on the roll actuation, as it is clear from experiments that the vast majority of the power is expended to actuate the roll motion.

\subsubsection{Application to Finnegan Actuator Design}

It is now possible to make a quantitative estimate of the actual velocity limit for a vehicle using four of the existing foil modules, with $0.40 \mathrm{~m} \times 0.10 \mathrm{~m}$ foils. In both configurations described above, all four foils are oriented with the foil thrust direction directly forward in the vehicle body frame. Defining a drag coefficient for the vehicle based on the vehicle projected frontal area, $A_{\text {vehicle }}$,

$$
C_{D}=\frac{D}{\frac{1}{2} \rho U^{2} A_{\text {vehicle }}}
$$

we find that at every attainable speed, the total thrust force produced by the foils must overcome the body drag,

$$
\begin{gathered}
T_{\text {tot }}=C_{T} \cdot\left(\frac{1}{2} \rho U^{2}\right) \cdot A_{\text {foils }}=C_{D} \cdot\left(\frac{1}{2} \rho U^{2}\right) \cdot A_{\text {vehicle }} \\
C_{T}=C_{D} \frac{A_{\text {vehicle }}}{A_{\text {foils }}}
\end{gathered}
$$

Hence, the maximum speed of the vehicle, as a function of the drag coefficient of the vehicle, is the maximum speed at which a foil actuator can produce the corresponding $C_{T}$, which is a function of the ratio of the surface area of the foil to the frontal area of the vehicle.

Figure 2-11 indicates how the inertial and the hydrodynamic limits on the actuator roll motor limit the maximum speed of the vehicle. The inertial limit is manifested as a limit on the maximum acceleration of the foil, which is set to a conservative value of $101 \frac{\mathrm{rad}}{\mathrm{s}^{2}}$ based on the maximum acceleration achieved by the actuator during 
tests with the $0.40 \mathrm{~m}$ foil in water. The hydrodynamic limit is a function of the power limitations of the actuator, which in this case stems from current limiting the roll motor amplifier to $12 \mathrm{~A}$ at $24 \mathrm{~V}$, and the manufacturer's estimate of $75 \%$ efficiency across the motor's two stage planetary gear head.

The power and acceleration requirements are extrapolated from 6 data points, representing 6 different sets of foil kinematics operating at a flow speed of $0.5 \mathrm{~m} / \mathrm{s}$. These kinematics were considered desirable because of their relatively low maximum current draw as a function of thrust coefficient, as experimentally demonstrated in the Towing Tank. The foil thrust coefficients range from 0.10 to 7.19, which correspond to vehicle drag coefficients of 0.08 to 5.86 given a ratio of foil area to vehicle frontal are of 0.8. The figures indicate that regardless of the vehicle drag coefficient, the primary limitation on the vehicle maximum speed is the power required to drive the foil at maximum velocity, which scales with $U^{3}$, rather than the torque required to accelerate the foil apparatus, which scales with $U^{2}$. Figure 2-12 plots the paths of the motor operation point with increasing speed on a torque-speed plot to indicate how the maximum power limit is approached when operating at each of the 6 selected thrust coefficients, where II is the nominal motor torque-speed curve at $24 \mathrm{~V}$, and I represents maximum power output of $201 \mathrm{~W}$. Each point to the right along a curve represents a vehicle speed increase of $0.25 \mathrm{~m} / \mathrm{s}$

For an extremely streamlined vehicle, a drag coefficient of 0.1 is attainable with difficulty, which would yield a vehicle maximum speed of greater than $2 \mathrm{~m} / \mathrm{s}$. Without a streamlined fairing, a drag coefficient between 0.8 and 1.4 is more appropriate, from Hoerner [29] based on the drag on a blunt cylinder with aspect ratio $\frac{l}{d}=4$, indicating a maximum speed under $1 \mathrm{~m} / \mathrm{s}$.

\subsection{Open Loop Vehicle Testing}

To determine the basic capabilities of the vehicle, Finnegan was deployed to the deep end of the MIT Alumni Pool (25 x 12 x 5 depth max. meters) and commanded to execute foil kinematics designed to actuate surge, sway, heave and yaw. The kinematics 
were executed with no state feedback to the parameters, although all sensor data was recorded to reconstruct vehicle performance. The vehicle was trimmed to be slightly negatively buoyant, stable in pitch and roll as close to level as possible. The vehicle was always tethered during the trials, with the cable consisting of standard CAT-5 cable ( 4 twisted pair conductors) either intertwined with the strands of a floating line (the final $10 \mathrm{~m}$, vehicle side) or wrapped around braided nylon line $(30 \mathrm{~m}$, operator side). With an attachment point above the center of gravity and a float at the appropriate location on the line, the tether configuration allowed the operator to set and maintain the unactuated rest position of the vehicle to a desired depth and position in the pool for all open loop performance trials. The experiments presented below were performed by Wolfe [72] and are described in further detail in (Wolfe, Licht et. al.) [73].

\subsubsection{Surge, Sway, Heave and Yaw Kinematics}

To test vehicle surge and yaw motion, lift based kinematics of the same form used in the testing and design analysis above were used. The roll and twist bias angles were zero, hence the foil motion was defined by:

$$
\begin{aligned}
& \phi(t)=\phi_{0} \sin (\omega t) \\
& \theta(t)=\theta_{0} \cos (\omega t)
\end{aligned}
$$

While surge and yaw used similar fin kinematics, the major difference between the two motions was the orientation of the foils. In the case of surge motion, all four foils faced forward, in the sense that the lift based kinematics above produced positive surge force, as in Figure 2-13: A. Yaw motion was generated using the same kinematics with the pair of foils on one side of the vehicle oriented to produce negative surge force, as in Figure 2-13: B.

The kinematics for sway and heave generation were sinusoidal as well, but the kinematics were outside of the range tested in the water tunnel and tow tank. In these "drag-based" kinematics, the foils were given a pitch bias of $\pi / 4$ or $-\pi / 4$ and a 


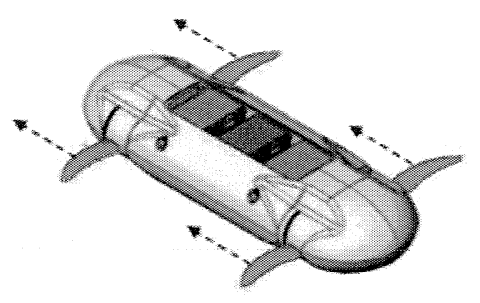

A: Surge

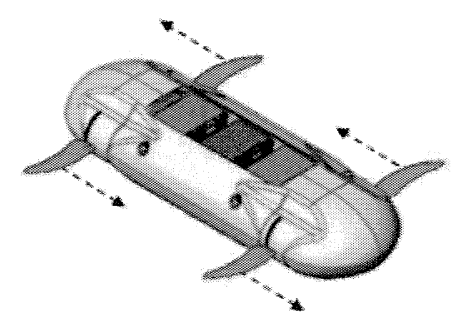

B: Yaw

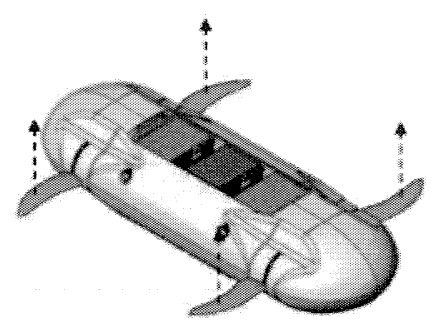

C: Heave

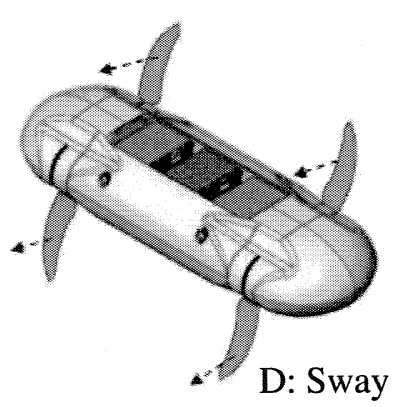

Figure 2-13: Foil orientation and force vectoring to actuate surge, sway, heave and yaw in open loop.

twist amplitude of $\pi / 4$. This created a sinusoidal rowing motion, generating a mean force nominally in the direction normal to the plane of the foil when $\theta=0$. In the case of heave, the foils are given no roll bias, so the equations of motion for heave are:

$$
\begin{gathered}
\phi(t)=\phi_{0} \sin (\omega t) \\
\theta(t)=\theta_{0} \cos (\omega t) \pm \pi / 4
\end{gathered}
$$

With a positive twist bias, the vehicle ascended, and with a negative twist bias the vehicle descended. Since there is still some relatively small horizontal thrust component with these kinematics, the front pair of foils was oriented backwards to counter the horizontal forces of the rear foils, as in Figure 2-13:C.

For sway, the foils were given roll bias or reorient the mean force. The foils on one side of the vehicle were given the maximum possible positive roll bias, while those on the other side were given the maximum negative roll bias, as shown in Figure 2-13:D. The kinematics for the foils in sway were: 


$$
\begin{gathered}
\phi(t)=\phi_{0} \sin (\omega t) \pm \phi_{\text {bias }} \\
\theta(t)=\theta_{0} \cos (\omega t) \pm \pi / 4
\end{gathered}
$$

Ideally, the roll bias would be $\pi / 2$, orienting the fins vertically, creating pure sideways force. However, the physical limitations on the actuators limited the maximum roll exursion to $\pm 80^{\circ}$. With this limitation, the mean force generated also had some small heave component.

\subsubsection{Performance Results}

\section{Lift Based Kinematics}

For surge testing, the oscillating frequency was held at $1 \mathrm{~Hz}$, with $\phi_{0}=\left[30^{\circ}, 45^{\circ}, 60^{\circ}\right]$, $\theta_{0}=\left[60^{\circ}, 40^{\circ}\right]$. Results, averaged over at least three trials for each case, are presented in Table 2.1. A maximum surge velocity $1.38 \mathrm{~m} / \mathrm{s}(0.69 \mathrm{BL} / \mathrm{s})[\mathrm{BL}=2.0 \mathrm{~m}$ with fairing as in the experiments detailed here, $1.3 \mathrm{~m} \mathrm{w} / \mathrm{o}$ fairing in Chapter 3] was recorded with $\phi_{0}=60^{\circ}$ and $\theta_{0}=40^{\circ}$. For every roll amplitude, the maximum velocity was higher for $\theta_{0}=40^{\circ}$, as expected given that a lower twist angle results in higher maximum angle of attack.

\begin{tabular}{|c|c|c|}
\hline$f=1.0 \mathrm{~Hz}$ & $\theta_{0}=60^{\circ}$ & $\theta_{0}=40^{\circ}$ \\
\hline$\phi_{0}=30^{\circ}$ & 0.31 & 0.45 \\
\hline$\phi_{0}=45^{\circ}$ & 0.44 & 0.63 \\
\hline$\phi_{0}=60^{\circ}$ & 0.61 & 0.69 \\
\hline
\end{tabular}

Table 2.1: Maximum Surge Velocities $[B L / s]$ vs. Kinematic Parameters

During yaw testing, the oscillating frequency was either 0.5 or $1 \mathrm{~Hz}$, with $\phi_{0}=$ $\left[45^{\circ} 60^{\circ}\right], \theta_{0}=\left[40^{\circ} 60^{\circ}\right]$. A maximum yaw velocity of $80.2^{\circ} / \mathrm{s}$ was recorded for $f=1 \mathrm{~Hz}$, $\phi_{0}=60^{\circ}$, and $\theta_{0}=60^{\circ}$, and is reported with results from all other tested parameters in Table 2.2. As with the reported surge data, each entry represents an average of maximum velocities. The most aggressive motion in the test matrix was beyond the capabilities of the vehicle (denoted by * in Table 2.2.) 


\begin{tabular}{|c|c|c|}
\hline $\mathrm{f}=0.5 \mathrm{~Hz} / \mathrm{f}=1.0 \mathrm{~Hz}$ & $\theta_{0}=60^{\circ}$ & $\theta_{0}=40^{\circ}$ \\
\hline$\phi_{0}=45^{\circ}$ & $32.2 / 69.9$ & $32.9 / 71.8$ \\
\hline$\phi_{0}=60^{\circ}$ & $34.7 / 80.2$ & $39.1 /^{*}$ \\
\hline
\end{tabular}

Table 2.2: Maximum Yaw Velocities [ $\% / s]$ vs. Kinematic Parameters

\section{Drag Based Kinematics}

For heave testing, oscillating frequency was either 0.5 or $1 \mathrm{~Hz}$, with $\phi_{0}=\left[15^{\circ} 30^{\circ} 45^{\circ}\right]$. As heave was actuated using the drag based kinematics, the pitch amplitude and pitch bias are fixed at $\theta_{0}=45^{\circ}$ and $\theta_{\beta}=45^{\circ}$. Results are shown in Table 2.3, with a maximum heave velocity of $0.4 \mathrm{~m} / \mathrm{s}$ achieved with $f=1 \mathrm{~Hz}, \phi_{0}=45^{\circ}$.

\begin{tabular}{|c|c|c|}
\hline$\phi_{0}$ & Fr $=0.5 \mathrm{~Hz}$ & Fr $=1 \mathrm{~Hz}$ \\
\hline $15^{\circ}$ & 0.04 & 0.09 \\
\hline $30^{\circ}$ & 0.07 & 0.15 \\
\hline $45^{\circ}$ & 0.11 & 0.20 \\
\hline
\end{tabular}

Table 2.3: Peak Heave Velocities $(B L / s)$

To actuate sway, roll bias, $\phi_{\beta}$, was introduced to the motion of all four foils. Due to the $\pm 80^{\circ}$ limit on the foil roll range, increasing the magnitude of $\phi_{\beta}$, in order to reduce the heave component of the resuting force vector, had the undesired effect of reducing the amplitude of $\phi_{0}$ to ensure that $\phi_{0}+\left|\phi_{\beta}\right|<80^{\circ}$. Compromise values of $\phi_{\beta}=54^{\circ}$ and $\phi_{0}=15^{\circ}$ were selected, with oscillating frequency of $1 \mathrm{~Hz}$ and 1.5 $\mathrm{Hz}$ (the frequency tested.) A maximum sway velocity of $0.34 \mathrm{~m} / \mathrm{s}(0.17 \mathrm{BL} / \mathrm{s})$ was achieved from $f=1.5 \mathrm{~Hz}$, and a sway velocity of $0.21 \mathrm{~m} / \mathrm{s}(0.11 \mathrm{BL} / \mathrm{s})$ was achieved for $f=1.0 H z$.

\subsection{Conclusion}

The vehicle performance that results from the feed-forward use of simple harmonic kinematics demonstrates that Finnegan meets the basic objectives of the biomimetic oscillating foil vehicle. With a maximum recorded speed of $1.38 \mathrm{~m} / \mathrm{s}$ (or $0.69 \mathrm{BL} / \mathrm{s}$ ) and maximum yaw rate of $80^{\circ} / \mathrm{s}$, as well as the ability to independently translate in surge and heave, the vehicle meets the goal of combining low speed agility with high 
speed swimming using the same actuator. The high authority thrust and maneuvering forces generated by isolated foils in towing tank and water tunnel tests effectively translate to propulsion of a free swimming vehicle.

The symmetric arrangement of the foils, with one pair forward and one pair aft, simplifies the choice of kinematics for open actuation of the vehicle. Just two basic styles are required to actuate four degrees of freedom; lift based thrust with twist motion upstroke-downstroke symmetric, and drag based propulsion with a $45^{\circ}$ twist bias, $45^{\circ}$ twist amplitude, to produce $90^{\circ}$ angle of attack on one half stroke combined with a completely feathered ( $0^{\circ}$ angle of attack) half stroke. In the following chapter, these two kinematic styles will be treated as extremes on a continuum, allowing for vectoring of thrust with the plane perpendicular to the mean direction of the foil axis. With closed loop control the ability of the same foils to actuate body pitch and roll will be demonstrated, and high speed maneuvering will be added to the low speed agility shown above. 


\section{Chapter 3}

\section{Control and Maneuvering with Harmonically Oscillating Foils}

\subsection{Introduction}

In this chapter, we demonstrate that foils can be used for closed loop control of an underwater vehicle in level flight and hovering as well as in large angle maneuvers such as banked and twisting turns. One of the major attractions of the 2DOF oscillating foil propulsor is that it can rapidly generate large maneuvering forces in a broad range of operating conditions. We show that these large maneuvering forces can be used to dramatically improve AUV performance by demonstrating obstacle avoidance at high speed, with a turning radius less than a half of the best reported for streamlined AUVs. A major contributing factor to these improvements is the operation of the vehicle while trimmed to be unstable in attitude, a development made possible by the use of speed independent, high bandwidth, high authority force vectoring of the foils to regulate attitude while hovering and cruising.

This chapter presents the advantages of the modified Rodrigues parameters as the attitude description underlying a control algorithm for large angle maneuvers, reviews stability results for attitude controllers based on the modified Rodrigues parameters, and presents the algorithm used to determine desired foil force production during hovering and cruising. 


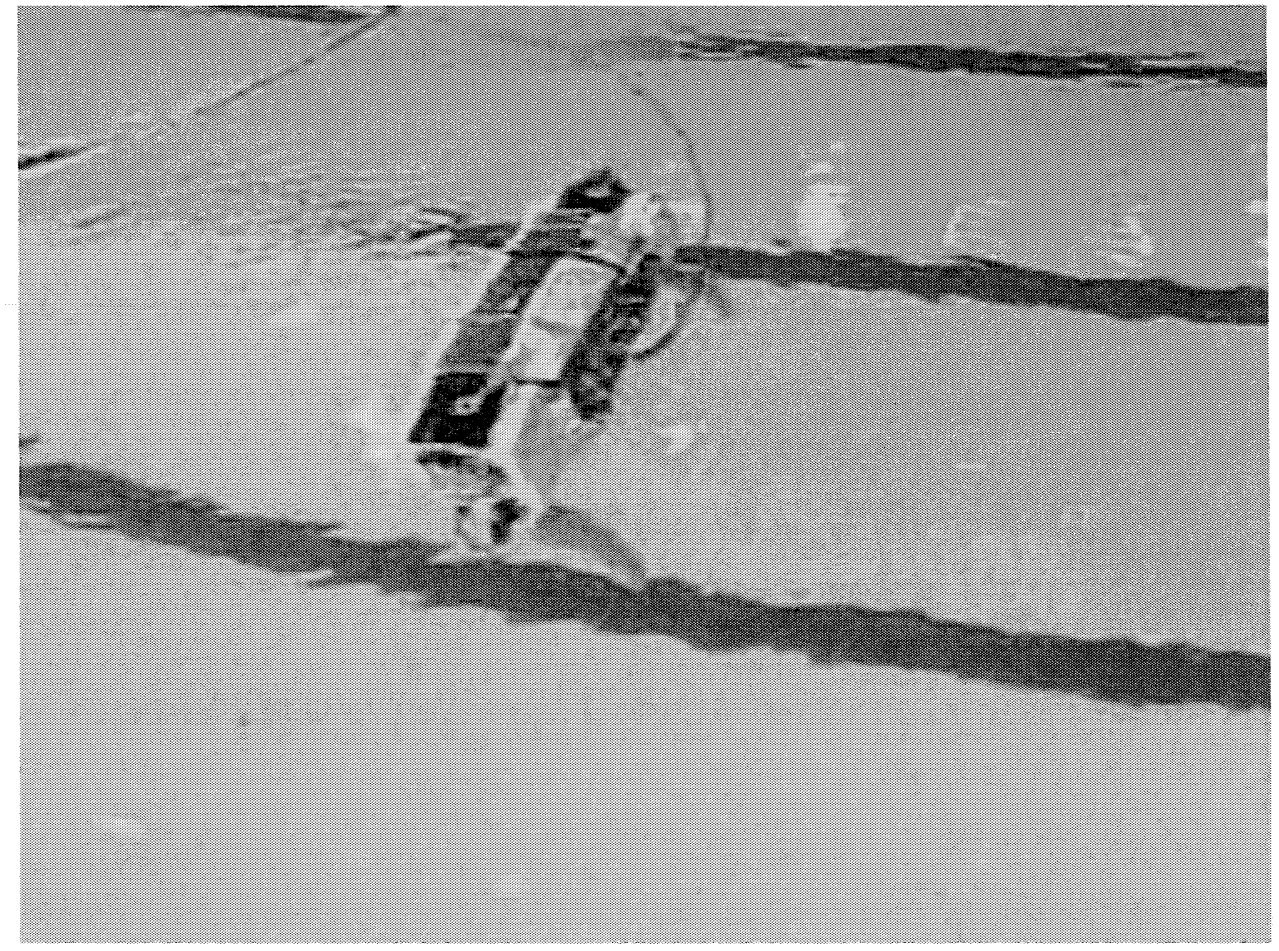

Figure 3-1: Screen capture from video of vehicle performing banked turn in the MIT Alumni Pool

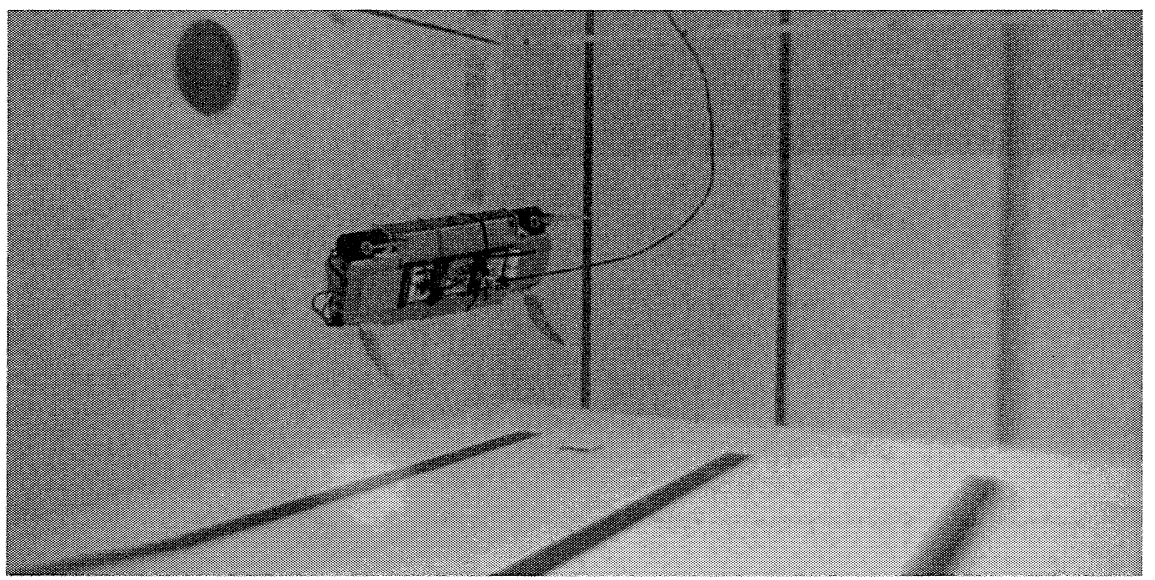

Figure 3-2: Screen capture from underwater video of vehicle performing banked turn in the MIT Alumni Pool 
Just three parameters of the harmonic oscillation are modified to vector forces from each of the foils in hovering and in cruising, with an algorithm motivated by data from experimental trials with bench mounted foils. Initial experiments with the dual joystick interface are used to tune the control parameters for large angle maneuvers. Finally, the results from maneuvering trials with Finnegan in the MIT Alumni Pool, focusing on rapid obstacle avoidance at speeds up to $9.8 \mathrm{~m} / \mathrm{s}(0.78$ $\mathrm{BL} / \mathrm{sec}$ ) are presented, with comparisons to the known performance of comparable underwater vehicles.

\subsection{Background}

Maneuverability and stability for marine animals has been identified as an important issue to be addressed within the biomimetic approach to underwater vehicle design. In the biological literature, discussions of stability are commonly approached in terms of stability in the horizontal and vertical planes during forward swimming, with body flexibility as one of the primary variables. [71] examines the role of body flexibility in the context of changes in animal stability in the horizontal and vertical planes during behaviors where body shape changes occur, such as turning, fast starting and aggressive swimming.

[15] attempts to determine constraints on maneuverability as a function of body flexibility, and [13] notes that stability can promote efficient locomotion in marine animals, but that the most highly maneuverable animals exhibit morphologies which tend to reduce stability, such as forward control surface locations and extremely flexible bodies. Amongst cetaceans, flexible bodies with forward control surfaces correspond to tight turning at low turning rates, useful for their "complex habitats," while more rigid bodies correspond to high turning rates but higher radii with greater speed in species in more open environments.

(The observation that morphology also correlates with prey type in [13] serve as an important reminder that the evolutionary pressures driving animal design do not necessarily correspond to the functional requirements of AUVs. This point is 
further reinforced by [69], which provides an overview of factors that contribute to fish maneuverability, emphasizing the highly complex nature of actuator dynamics and kinematics, along with the need for a clear a priori statement laying out the functional requirements for vehicles hoping to profile biological inspiration.)

The tradeoff between maneuverability and stability is found in the design of man made underwater vehicles as well, both in terms of forward swimming stability and of attitude stability. Rigid bodies remain the norm in underwater vehicle design for reasons to do with materials, actuation, general complexity, and payload space, hence control surface placement is the primary concern in forward swimming stability for underwater vehicles. Control surfaces are generally placed towards the rear of the vehicle to enhance stability in the horizontal and vertical planes.

In some cases, extremely high attitude stability is a functional requirement, e.g. the SeaBed vehicle [58], tasked for high resolution imaging, which uses two widely spaced cylindrical hulls, one negatively buoyant and one positively buoyant, to effect a large separation between center of buoyancy and center of gravity. The streamlined torpedo shape that forms the basis for the design of most operational AUVs is inherently less stable in pitch and roll, and has much lower damping in roll, than multi-hulled vehicles. Typically, however, this reduction in stability is treated as a necessary evil brought on by the need for reduced drag [26], and the metacentric height is kept as large as possible. Attitude stability does simplify the control design significantly; at the simplest level, decoupled pitch and yaw control strategies with unactuated roll axis can be successful, as in [59]. Indeed, as shown in the testing of the basic open loop performance properties of Finnegan in Chapter 1, passive stability can even eliminate the need for attitude regulation in some circumstances.

Finnegan achieves high maneuverability in part through passive attitude instability. While this instability requires constant control effort to regulate attitude in the face of disturbances, it allows for rapid control to arbitrary attitudes without the need to offset a large hydrostatic wrench, which is particularly important when the same actuator must be used to generate both forward propulsion and roll/pitch moments. The placement of the foils has the effect of lowering stability in one vehicle plane 


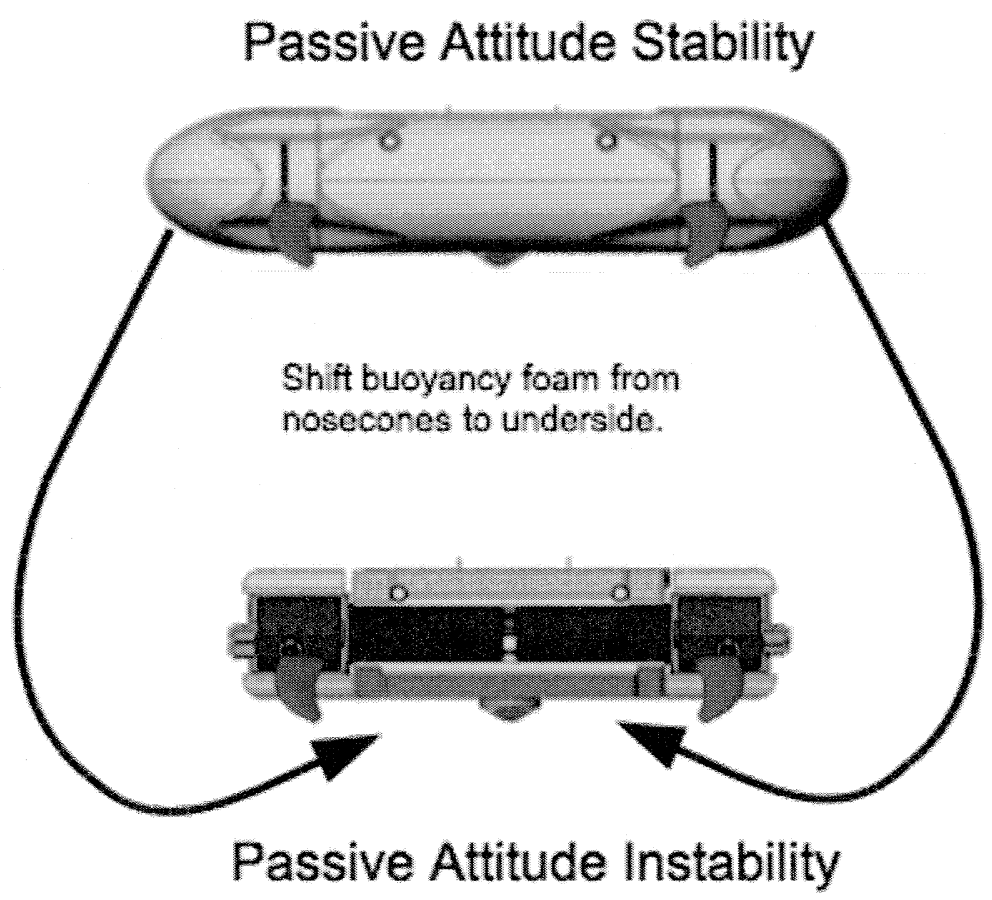

Figure 3-3: The vehicle trim configuration is changed to reduce attitude stability by rearranging the buoyancy foam. The nose and tail cones are removed, and replaced with buoyancy located below the vehicle center of gravity. Without the nose cones, removal of the flooded fairing eliminates a large volume of captured water from from the effective mass of the vehicle, further promoting maneuverability. The vehicle unactuated equilibrium position with the fairing is illustrated in Figure 3-4. The vehicle unactuated equilibrium position in unstable trim configuration without the fairing is shown in Figure 3-5

during forward swimming, and the use of large angle maneuvers allows the vehicle to orient this low stability plane favorably for aggressive maneuvering.

\subsection{Attitude Regulation using the Modified Ro- drigues Parameters}

In the absence of a vehicle or foil model which covers all states that may be encountered in an aggressively maneuvering vehicle, control algorithms dependent on detailed knowledge of system dynamics were deemed to be unattractive. At the same 


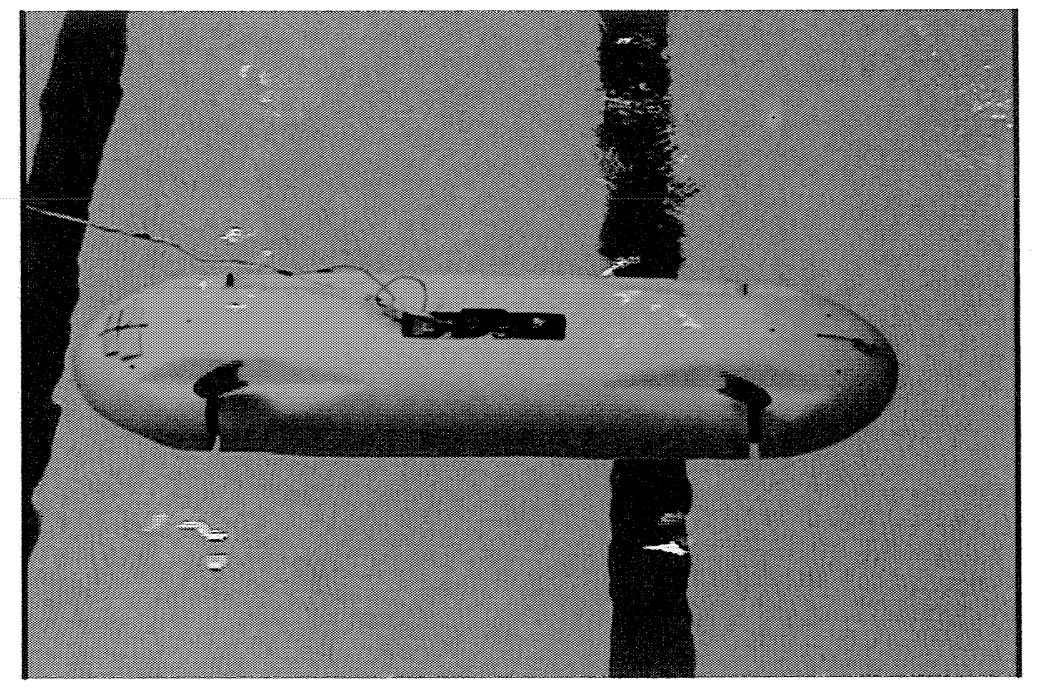

Figure 3-4: Vehicle unactuated equilibrium position in stable trim configuration (i.e. with nose cones).

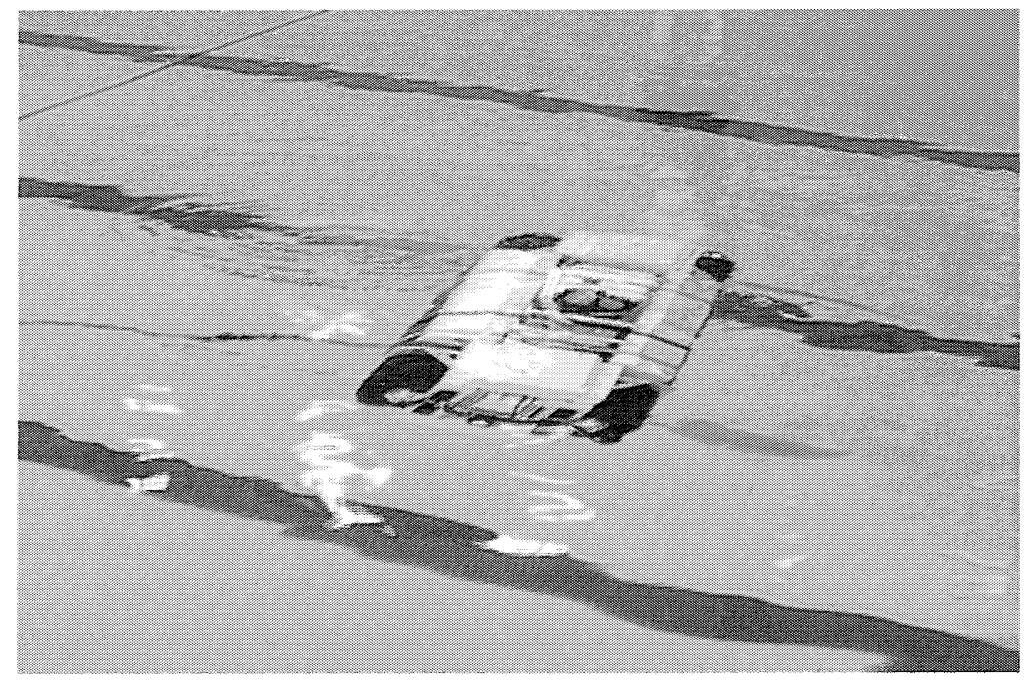

Figure 3-5: Vehicle unactuated equilibrium position in unstable trim configuration (i.e. without nose cones). The buoyancy used to replace the nose cone buoyancy volume can be seen running the lenght of the vehicle on either side of the DVL (the orange transducer faces indicate the central location of the upturned DVL.) 
time, oversimplified heuristic control methods which rely on the small angle assumption while using Euler angles are bound to fail in a vehicle performing large angle maneuvers.

For the present work, a heuristic attitude controller was adopted, linear in the Modified Rodrigues Parameters, which was proposed in Tsiotras [66], with stability results for underwater vehicles that do not rely on explicit knowledge of vehicle inertial or hydrodynamic properties explored in both Boskovic [8] and Fjellstad [19] . The resulting controller specifies desired actuator moment as a function of Modified Rodrigues parameters, the body fixed rotation, and two matrices of control gains.

The modified Rodrgiues Parameters representation is a three parameter description of attitude which takes advantage of Euler's Theorem that every orientation of a rigid body can be reached from any other orientation of that body through a finite rotation, with magnitude described by a the principle angle, $\gamma$, about a constant body fixed principal axis, $\lambda$. As noted by [8], minimal three-parameter descriptions of attitude can simplify the development of control laws, but all three-parameter descriptions introduce singularities. MRPs effectively reduce the singularity to a single physical configuration, at $\gamma=2 \pi$ by defining the parameters according to:

$$
\rho=\lambda \arctan \left(\frac{\gamma}{4}\right)
$$

The modified Rodrigues Parameters differ from the Cayley-Rodrigues parameters only in the use of a factor of four in the denominator of the arc tangent term, rather than a two.

The advantage of control using the Modified Rodrigues parameters, or other representations such as quaternions, over the more common Euler angles, is that it circumvents the kinematic non-linearities inherent in descriptions based on ordered rotations for large angle maneuvers. Essentially, the use of the modified Rodrigues parameters makes determining a smooth trajectory between set points trivial, since the constant axis of rotation required is inherent in the definition of the parameters. Admittedly this trajectory cannot be assumed to be optimal in the sense of time or 
effort. Framed in terms of the Euler angles, the problem of finding $\omega(t)$ to follow a desired some desired trajectory within the Euler angles can be written explicitly as:

$$
\frac{d E}{d t}=\left[\begin{array}{ccc}
1 & \sin \phi \tan \theta & \cos \phi \tan \theta \\
0 & \cos \phi & -\sin \phi \\
0 & \sin \phi / \cos \theta & \cos \phi / \cos \theta
\end{array}\right] \omega
$$

but this leaves the problem of determining of the smooth trajectory required to move from an arbitrary state to some arbitrary Euler angle set point. If the rotation can be described with a single non-zero Euler angle (i.e. pure yaw, pure pitch, or pure roll,) with no disturbances and perfectly modeled acutation, then the problem is trivial. If a large angle rotation is required, however, the introduction of even a small non-zero value in the other components of a desired set point, or perturbation in the vehicle state off the desired trajectory requires significant Euler angle trajectory recalculation. The most common solution to this problem in applications with three rotational degrees of freedom is through the use of quaternions, a four parameter attitude representation which has the advantage of eliminating singularities entirely.

Vector rotations using quaternions are computational more efficient compared to both Euler angles and the Cayley-Rodrigues and Modified Rodrigues parameters. With the time scale of vehicle and foil action, and the update rates of the various attitude and position sensing devices, the computational load for state estimation and trajectory generation is negligible. This is especially true given that very few vectors are being updated through rotational transformations, in constrast to applications such as high speed animation for gaming and virtual reality, where success depends on visually smooth rotations of the large number of vectors required to make up a high definition scene.

Given that the computational efficiency is unimportant, control in the MRP has the advantage in that it provides an intuitively straightforward description of the trajectory between attitude setpoints. There is no barrier to providing the attitude set points themselves in the Euler angles, which are relatively easy to visualize statically, and then calculating the kinematics using the axis-angle approach of the MRP. 
In an underwater vehicle commanded either through a direct real time interface or through scripted command sequences, this eases the task of constructing maneuvering sequences through attitude setpoints to effectively take advantage of external knowledge of vehicle, actuator and fluid dynamics.

Tsiotras [66] show that a kinematic regulator using only terms linear in the MRP and angular rates, i.e.:

$$
\tau=-k_{1} \vec{\rho}-k_{2} \vec{\omega}
$$

is globally asymptotically stable in the sense of Lyapunov for positive gains $k_{1}$ and $k_{2}, \rho$ is the vector of the modified Rodrigues Parameters, and $\omega$ is the vector of body angular rotation rate, using as a Lyapunov function

$$
\begin{gathered}
V=\frac{1}{2} \omega^{T} J \omega+k_{1} W(\rho) \\
W(\rho)=1-\ln \left(1+\rho^{T} \rho\right)
\end{gathered}
$$

Fjellstad [19] and later Boskovic [8] extended these results to dynamic control of underwater vehicles using the standard model of a hovering underwater vehicle from Fossen [21], which combines the equations of motion for 6 DOF rigid body:

$$
\begin{aligned}
& m\left[\dot{v}+\omega \times v+\dot{\omega} \times r_{g}+\omega \times\left(\omega \times r_{g}\right)\right]=f \\
& \left.J \dot{\omega}+\omega \times(J \omega)+m r_{g} \times(\dot{(} v)+\omega \times v\right)=\tau
\end{aligned}
$$

with the form of the hydrodynamic forcing to find dynamic equations of the form:

$$
M \dot{v}+C(v) x+D(v) v+g(x)=u
$$

where $M$ is a positive definite matrix representing inertia of the vehicle mass and added fluid mass, $C$ is a skew symmetric matrix of Coriolis and centripetal terms, and $D$ is a damping matrix representing fluid damping. $g(x)$ is the hydrostatic wrench entirely dependent on attitude, while $u$ is the external linear and angular forcing from 


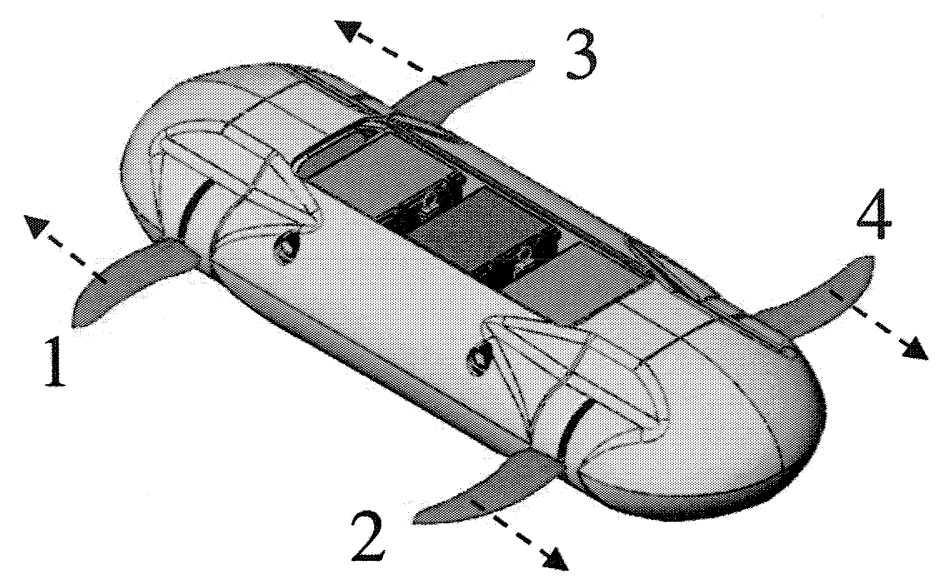

Figure 3-6: Foil mean position for hovering tasks. If $\phi_{0}$ and $\theta_{0}$ are identical for all four foils, with $\theta_{\beta}=0$, the mean forces from the fore and aft foils are directly opposed, resulting in zero nominal force and torque on the body.

disturbances and acutation. The stability of several controllers that are non-linear in the Cayley-Rodrigues and modified Rodrigues parameters is proven, and Boscovic [8] proves the stability of the linear controller given in (3.3). These stability results rely only on the form of the equation, in particular the positive definiteness of mass matrix and the skew symmetry of the Coriolis and centipetal terms, and as a results are independent of vehicle parameters.

\subsubsection{Foil Force Vector Command}

The magnitude and direction of the total torque and force requirements are generated by the control algorithm as a function of the vehicle state and the desired vehicle state. The resulting force desired from each foil is determined by separately calculating a vector of lift forces and a vector of thrust forces. By exploiting symmetry and using of differential actuation, we can do the calculations separately, since only lift is used to generate heave force, and roll and pitch torque, while only thrust is used to generate surge force and yaw torque.

During both hovering and cruising, the left and right foil pairs are used differentially to generate roll torque through lift, while the fore and aft foil pairs are used 


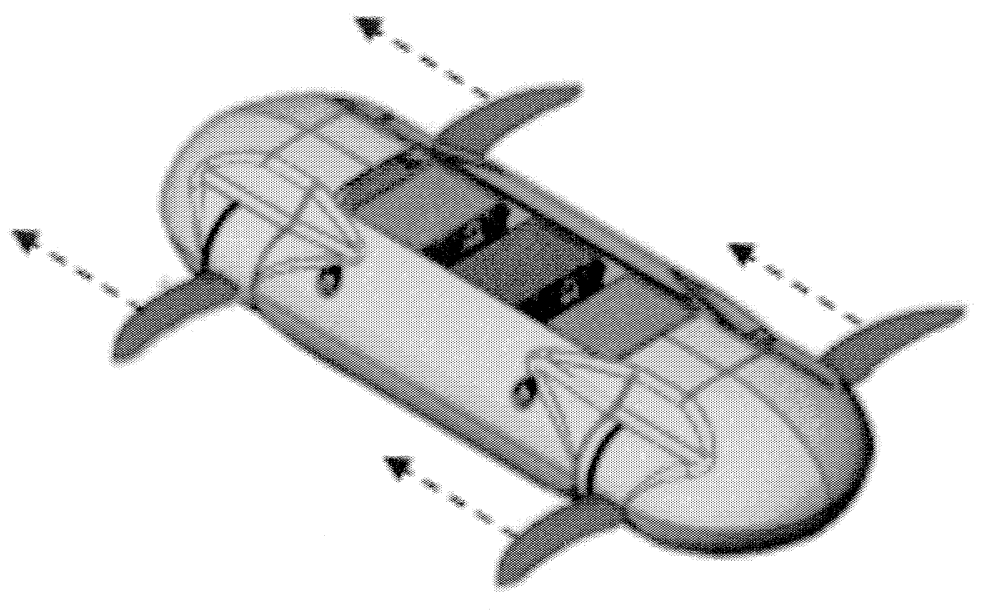

Figure 3-7: Foil mean position for cruising. If $\phi_{0}$ and $\theta_{0}$ are identical for all four foils, with $\theta_{\beta}=0$, the mean forces from the fore and aft foils all contribute to drive the vehicle in, with zero nominal heave and sway force, and zero torque on the body.

to differentially generate pitch torque through lift. During cruising, all four foils are oriented in the same direction and generate a positive baseline surge force. The total surge force is controlled by adding or subtracting thrust on all foils, while the yaw moment is generated by differentially changing the thrust on the left and right foil pairs. The foil position in hover and in cruise are shown in Figures 3-6 and 3-7 with the baseline forces (i.e the foil force vector with all control inputs zero) represented by the dotted arrows.

During hovering, the aft foils are reversed by adding a $180^{\circ}$ offset to the twist position. As a result, the fore and aft foil pairs are always thrusting against each other, such that an increase in thrust from the forward pair and/or a decrease in thrust from the aft pair results in forward motion, and the opposite results in motion aft. Foils on opposite corners are generate yaw moment of the same sign. Hence the fore-left and aft-right foils are paired against the fore-right and aft-left foils to differentially generate yaw torque through thrust, and the fore and aft foil pairs are 
used to differentially generate surge force through thrust. Finally, all four foils are recruited to generate force in the heave direction when the vehicle is at or near level.

The total lift and thrust demanded from each foil during cruising can be summarized as:

$$
\begin{array}{ll}
L_{1}=\frac{\tau_{1}}{4}+\frac{\tau_{2}}{4} & T_{1}=T-\frac{\tau_{3}}{4}+\frac{u_{1}}{4} \\
L_{2}=-\frac{\tau_{1}}{4}+\frac{\tau_{2}}{4} & T_{2}=T+\frac{\tau_{3}}{4}+\frac{u_{1}}{4} \\
L_{3}=+\frac{\tau_{1}}{4}-\frac{\tau_{2}}{4} & T_{3}=T-\frac{\tau_{3}}{4}+\frac{u_{1}}{4} \\
L_{4}=-\frac{\tau_{1}}{4}-\frac{\tau_{2}}{4} & T_{4}=T+\frac{\tau_{3}}{4}+\frac{u_{1}}{4}
\end{array}
$$

where $\tau_{1}, \tau_{2}, \tau_{3}$ are the components of the desired torque vector $\vec{\tau}$, and $u_{1}, u_{2}, u_{3}$ are the components of the desired linear force. Subscripts refer to foils as enumerated in Figure 3-6. $T$ with no subscript represents the mean thrust generated by a foil following the unadulterated baseline kinematics. Positive lift is always in the positive z-direction with respect to the body. Positive thrust direction is determined by the actuator orientation (i.e. with forward orientation, positive thrust is in the positive $\mathrm{x}$-direction with respect to the body, with reverse orientation, positive thrust is in the negative $\mathrm{x}$-direction.)

The total lift and thrust demanded from each foil during hovering can be summarized as:

$$
\begin{array}{ll}
L_{1}=\frac{\tau_{1}}{4}+\frac{\tau_{2}}{4}+\frac{u_{3}}{4} & T_{1}=T-\frac{\tau_{3}}{4}+\frac{u_{1}}{4} \\
L_{2}=-\frac{\tau_{1}}{4}+\frac{\tau_{2}}{4}+\frac{u_{3}}{4} & T_{2}=T+\frac{\tau_{3}}{4}+\frac{u_{1}}{4} \\
L_{3}=+\frac{\tau_{1}}{4}-\frac{\tau_{2}}{4}+\frac{u_{3}}{4} & T_{3}=T+\frac{\tau_{3}}{4}-\frac{u_{1}}{4} \\
L_{4}=-\frac{\tau_{1}}{4}-\frac{\tau_{2}}{4}+\frac{u_{3}}{4} & T_{4}=T-\frac{\tau_{3}}{4}-\frac{u_{1}}{4}
\end{array}
$$

Control of the vehicle was accomplished using force vectoring from the four foils 
to produce the moments dictated by (3.3) to drive the vehicle to attitude and depth set points. The set points as a function of time were defined through direct user input from a joystick control system, or from timed execution of scripted mission, or both, as detailed below after an explanation of the thrust vectoring algorithm for body torque production

\subsection{Foil Force Vectoring}

Vehicle closed loop control as described above is predicated on the ability to produce lift and thrust changes on each foil through perturbations about a set of baseline kinematics. For open loop testing, a distinction was made between lift based and drag based kinematics, where the roll and twist bias angles, $\phi_{\beta}$ and $\theta_{\beta}$, were zero for lift based kinematics, and $\theta_{\beta}$ of $\pi / 4$ or $-\pi / 4$ was used for drag based kinematics. These "pure" lift and drag based kinematics generate mean force vectors approximately perpendicular to one another.

To achieve the force vectoring capabilities required for closed loop control of the vehicle, pure lift and drag based kinematics should be thought of as extremes on a continuum of possible foil kinematics, regulated by the twist bias, $\theta_{\beta}$. The harmonic foil kinematics used for all of the open loop tasks in Chapter 2 need only a slight modification to vector foil thrust for use in closed loop control. The basic motion of the foils is again sinusoidal in both roll $(\phi)$ and twist $(\theta)$, with twist motion leading roll motion by 90 degrees.

$$
\begin{gathered}
\phi(t)=\phi_{0} \sin (\omega t)+\phi_{\beta} \\
\theta(t)=\theta_{0} \cos (\omega t)+\theta_{\beta}
\end{gathered}
$$

where $\phi_{0}$ and $\theta_{0}$ are angular amplitudes, and $\phi_{\beta}$ and $\theta_{\beta}$ are constant angular biases.

As described below, for both hovering and cruising tasks, perturbations in $\phi_{0}, \theta_{0}$ and $\theta_{\beta}$ about well chosen baseline kinematics are the only inputs needed to vector foil thrust. Assuming these perturbations in the kinematics are small, the average 
lift, $\bar{L}$, and average thrust, $\bar{T}$, over a full cycle can be linearized about the baseline operating point as:

$$
\begin{aligned}
\bar{L} & =f\left(\phi_{0}, \theta_{0}, \theta_{\beta}\right) \\
& =L_{0}+\left.\Delta \phi_{0} \frac{\partial \bar{L}}{\partial \phi_{0}}\right|_{\theta_{0}, \theta_{\beta}}+\left.\Delta \theta_{0} \frac{\partial \bar{L}}{\partial \theta_{0}}\right|_{\phi_{0}, \theta_{\beta}}+\left.\Delta \theta_{\beta} \frac{\partial \bar{L}}{\partial \theta_{\beta}}\right|_{\phi_{0}, \theta_{0}} \\
\bar{T} & =f\left(\phi_{0}, \theta_{0}, \theta_{\beta}\right) \\
& =T_{0}+\left.\Delta \phi_{0} \frac{\partial \bar{T}}{\partial \phi_{0}}\right|_{\theta_{0}, \theta_{\beta}}+\left.\Delta \theta_{0} \frac{\partial \bar{T}}{\partial \theta_{0}}\right|_{\phi_{0}, \theta_{\beta}}+\left.\Delta \theta_{\beta} \frac{\partial \bar{T}}{\partial \theta_{\beta}}\right|_{\phi_{0}, \theta_{0}}
\end{aligned}
$$

\section{Force Vectoring at Cruising Speed}

Haugsdal [27] demonstrated a linear relationship between pitch bias for harmonically heaving and pitching foils and maneuvering force, i.e. mean force perpendicular to the incoming flow. To test whether such a relationship holds for rolling and twisting foils, Flores [20] mounted the prototype of the initial foil module design module under a six-axis dynamometer in the MIT water tunnel. The design, illustrated in Figure 38 , was deemed unlikely to succeed on the vehicle itself, primarily due to the limited range of motion on the roll axis $\left( \pm 15^{\circ}\right)$ However, the size of the tunnel cross-section imposed an even greater restriction on foil motion for any foil of reasonable size, and so the module design problems did not limit the usefulness in testing. Details of the tunnel actuator construction and experimental apparatus can be found in Flores [20].

Along with extensive testing of pure thrust producing modes across a range of $S t$ number and $\alpha_{\max }$ with flow speeds between 0.4 and $1.0 \mathrm{~m} / \mathrm{s}$, the production of maneuvering forces through the addition of twist bias, $\theta_{\beta}$, to the foil twist angle throughout the entire propulsive stroke.

The experiments were performed at 5 points in the sample space of Strouhal number and $\alpha_{\max }$ where relatively high thrust coefficients were observed. Bias angles from $-10^{\circ}$ up to $40^{\circ}$ in $5^{\circ}$ increments were tested at $S t$ of 0.4 and 0.7 and $\frac{h_{0.7}}{c}$ values

of 1.0 and 1.5. Bias angles from $-10^{\circ}$ up to $30^{\circ}$ were tested at $S t=0.4$ and $\frac{h_{0.7}}{c}=0.5$. $\alpha_{\text {max }}$ for all tests was held at $40^{\circ}$.

In each case, as shown in Figure 3-9, this relatively simple twist bias strategy 


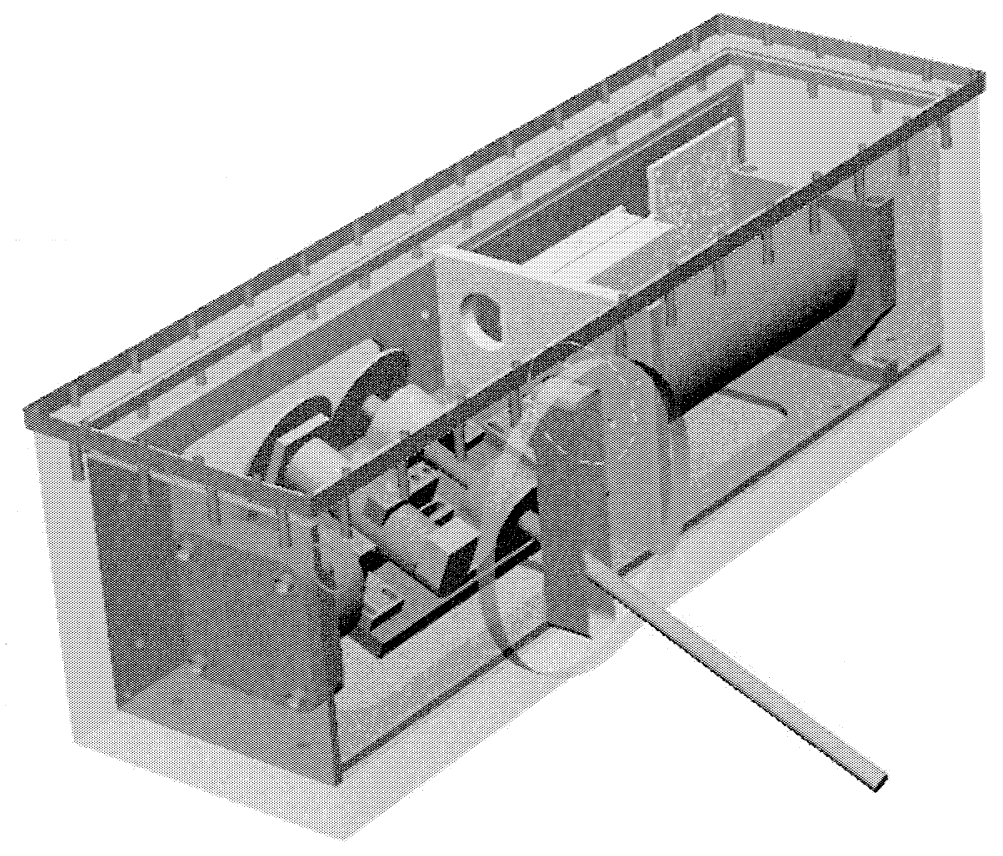

Figure 3-8: Single housing actuator design. Foil shaft protrudes through a slot in the Lexan box housing all components. Seal was accomplished with a flexible bellows. This design was used for testing in the MIT water tunnel where foil motion was restricted by flow section, but the range of motion in roll was deemed to be too limited for use on the vehicle.

resulted in a near linear relationship between lift coefficient and bias angle. A similar relationship was also found in experiments with two dimensional (heaving and twisting) foils in the MIT testing tank by Haugsdal [27]. Much higher lift coefficients were available in this manner than with the foil acting as a traditional, non-flapping control surface, with mean lift coefficients over 3 recorded in two cases, at $\theta_{\text {bias }}$ of $25^{\circ}$ and $30^{\circ}$, in contrast to a maximum lift coefficient of 0.5 before stall for the static foil.

Figure 3-10 plots the lift coefficient against the drag coefficient for each of the five sets of kinematics. The effect of higher amplitudes is seen to be much less significant than the Strouhal number in determining the location and shape of the curve. Note that maximum angle of attack is held constant throughout. The region encompassed by the far right hand curve in Figure 3-10 can be interpreted as the zone of (normalized) force vectors that are known to be available to the given actuator 


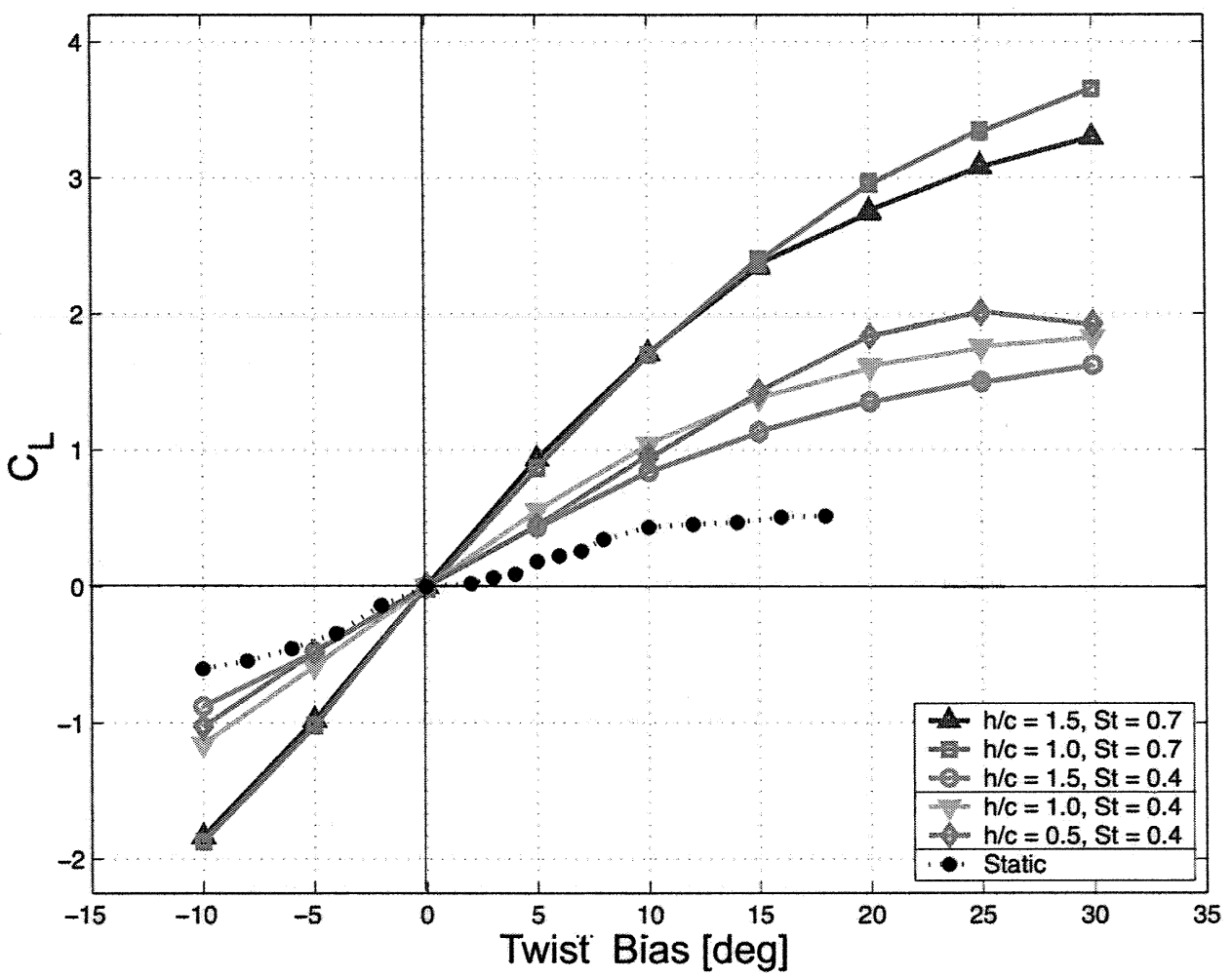

Figure 3-9: Mean $C_{L}$ v. twist bias $\theta_{\text {beta }}$ Data from experiments performed by Flores [20] and previously published in [43].

and foil pair while the vehicle is cruising at the test speed. Even without testing the actuator to its absolute limit, it is clear that powerful turning forces can be generated while braking or thrusting. (Testing with 2-D foils suggests that pure braking forces are also available simply by changing the phasing of the pitching and heaving motions [55].)

In crusing, thrust and required torque increase in tandem as the maximum angle of attack is increased. The maximum thrust available and the corresponding twist amplitude is a strong function of the vehicle forward speed, foil frequency and roll amplitude. For a given foil frequency, as the vehicle speed increases the twist amplitude can be decreased to avoid a decrease in the maximum angle of attack, thus maintaining thrust. With Finnegan, a typical acceleration to speeds of greater than $0.5 \mathrm{BL} / \mathrm{s}$ with constant $\phi_{0}$ and constant $f$ requires that the value of $\theta_{0}$ when accelerating from rest must be greater than $\theta_{0}$ used for maximum speed. Using the twist amplitude required to travel at speed, but with no vehicle velocity, would result in 


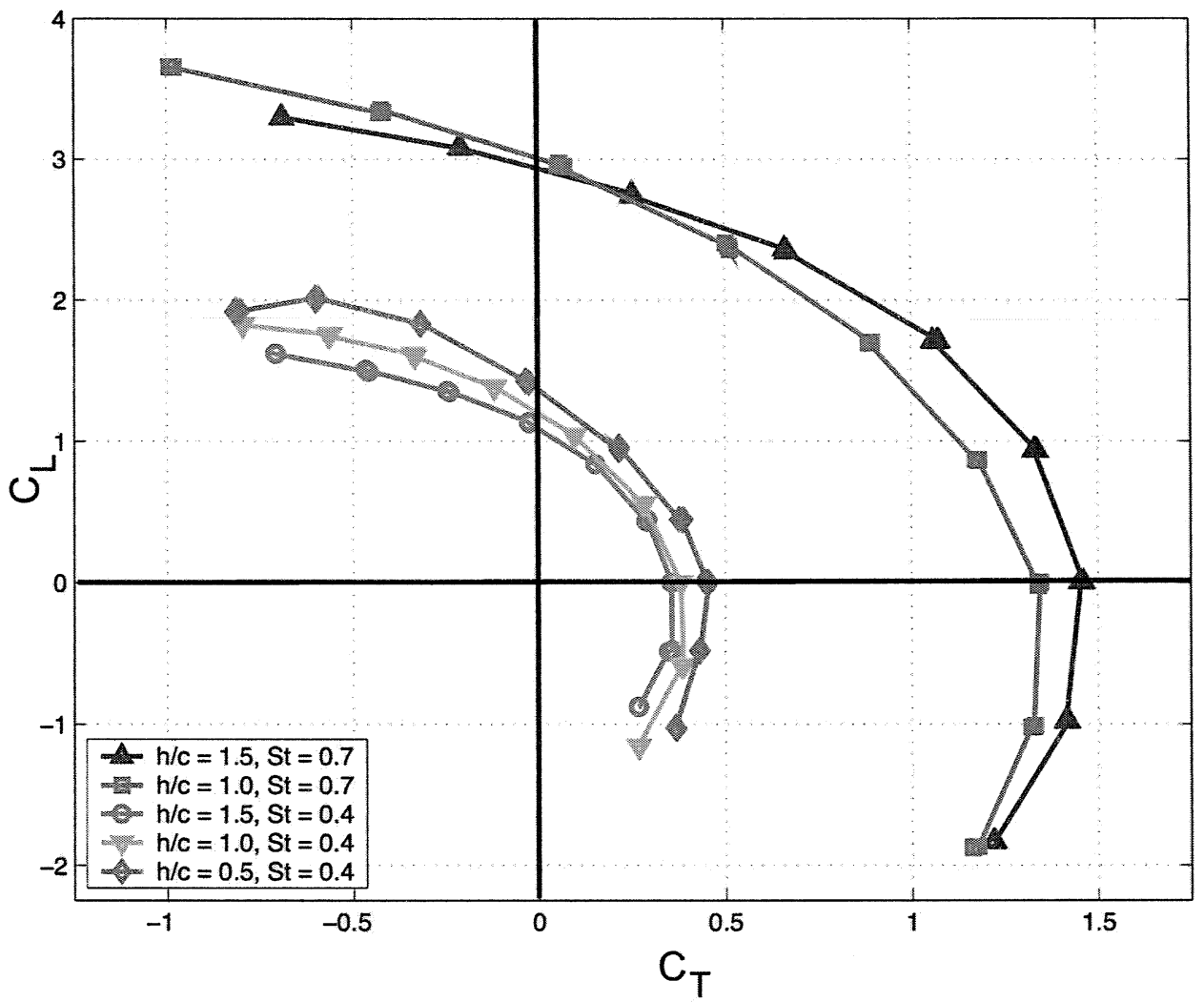

Figure 3-10: Effect of twist bias $\theta_{\beta}$ on $C_{L}$ and $C_{T}$. Data from experiments performed by Flores [20] and previously published in [43].

extreme angles of attack, generating little thrust while exceeding the actuator torque capacity due to excessive lift force on the foil.

Once a steady cruising speed has been reached for a given set of cruising foil kinematics, lift and thrust from all foils can be controlled through perturbations in $\phi_{0}$ and $\theta_{\beta}$. With $\Delta \theta_{0}=0$, the foil lift and thrust are of the form:

$$
\begin{aligned}
& \bar{L}=L_{0}+\Delta \theta_{\beta}\left|\frac{\partial \bar{L}}{\partial \theta_{\beta}}\right|_{\phi_{0}, \theta_{0}} \mid \\
& \bar{T}=T_{0}+\left.\Delta \phi_{0}\left|\frac{\partial \bar{T}}{\partial \phi_{0}}\right|_{\theta_{0}, \theta_{\beta}}\left|-\Delta \theta_{0}\right| \frac{\partial \bar{T}}{\partial \theta_{0}}\right|_{\phi_{0}, \theta_{\beta}} \mid
\end{aligned}
$$


given that;

$$
\begin{aligned}
& \left.\frac{\partial \bar{L}}{\partial \theta_{0}}\right|_{\phi_{0}, \theta_{\beta}} \simeq 0 \\
& \left.\frac{\partial \bar{L}}{\partial \theta_{\beta}}\right|_{\phi_{0}, \theta_{0}}>0 \\
& \left.\frac{\partial \bar{T}}{\partial \phi_{0}}\right|_{\theta_{0}, \theta_{\beta}}>0 \\
& \left.\frac{\partial \bar{T}}{\partial \theta_{\beta}}\right|_{\phi_{0}, \theta_{0}} \simeq 0
\end{aligned}
$$

Vehicle yaw is actuated through differential changes in foil thrust, hence yaw is actuated entirely through changes in $\phi_{0}$. For each foil:

$$
\phi_{0_{i}}=\phi_{0}+\left(T_{i}-T\right) k_{T}^{c}
$$

where the scalar $k_{T}^{h}>0$ is a heuristically tuned feedback gain,and the subscript $i$ corresponds to the foil as in (3.9). Twist bias is perturbed from a baseline of $0^{\circ}$ to control foil lift according to:

$$
\theta_{\beta, i}=L_{i} k_{L}^{c}
$$

where the scalar $k_{L}^{c}$ is a heuritically tuned feedback gain. Combining (3.9), (3.15), and (3.16), and holding all other parameters constant, the desired foil kinematics for cruising are: 


$$
\begin{array}{cr}
f_{1-4}=f & \\
\theta_{0_{1-4}}=\theta_{0} & \\
\phi_{\beta_{1-4}}=0 & \theta_{\beta_{1}}=k_{L}^{c}\left(\frac{\tau_{1}}{4}+\frac{\tau_{2}}{4}\right) \\
\phi_{0_{1}}=\phi_{0}+k_{T}^{c}\left(-\frac{\tau_{3}}{4}+\frac{u_{1}}{4}\right) & \theta_{\beta_{2}}=k_{L}^{c}\left(-\frac{\tau_{1}}{4}+\frac{\tau_{2}}{4}\right) \\
\phi_{0_{2}}=\phi_{0}+k_{T}^{c}\left(\frac{\tau_{3}}{4}+\frac{u_{1}}{4}\right) & \theta_{\beta_{3}}=k_{L}^{c}\left(\frac{\tau_{1}}{4}-\frac{\tau_{2}}{4}\right) \\
\phi_{0_{3}}=\phi_{0}+k_{T}^{c}\left(-\frac{\tau_{3}}{4}+\frac{u_{1}}{4}\right) & \theta_{\beta_{4}}=k_{L}^{c}\left(-\frac{\tau_{1}}{4}-\frac{\tau_{2}}{4}\right) \\
\phi_{0_{4}}=\phi_{0}+k_{T}^{c}\left(\frac{\tau_{3}}{4}+\frac{u_{1}}{4}\right) &
\end{array}
$$

\subsubsection{Hovering}

For the hovering case, the baseline value of twist amplitude, $\theta_{0}^{H}$, should be set to produce thrust with low mean lift, and hence low actuator torque demand, to avoid unnecessary energy expenditure and oscillating disturbance on the vehicle. For differential thrust to effectively actuate yaw, it must be possible both to increase and decrease thrust, so the baseline thrust value be at some midrange between zero thrust and maximum thrust.

In addition to testing the vehicle actuator thrust production at cruising speed as described in the previous chapter, Polidoro [53] also performed tests of foil lift and thrust force with no incoming flow, to test actuator capabilities during vehicle hovering tasks, finding that for a given $f$ and $\phi_{0}$, hence constant $\dot{\phi}_{\text {max }}$, the mean hovering thrust peaks at a value of $\theta_{0}$ near $\pi / 4$.

Given that the baseline kinematics must not produce maximum thrust, the value of $\theta_{0}$ must either be in the range $0<\theta_{0}<\pi / 4$ or $\pi / 4<\theta_{0}<\pi / 2$. Polidoro [?] also found that the lift produced during a single half stroke monotonically increases with decreasing $\theta_{0}$ for $0<\theta_{0}<\pi / 2$. Based on the desire to limit the amplitude of the oscillating lift force for the baseline kinematics, $\theta_{0}^{H}$ should be between $\pi / 4$ (maximum thrust) and $\pi / 2$ (fully feathered foil). 
Lift and thrust can then be independently controlled with perturbations in $\theta_{0}$ and $\theta_{\beta}$. With $\Delta \phi_{0}=0$, lift and thrust are of the form:

$$
\begin{aligned}
& \bar{L}^{H}=L_{0}^{H}+\Delta \theta_{\beta}\left|\frac{\partial \bar{L}}{\partial \theta_{\beta}}\right|_{\phi_{0}, \theta_{0}} \mid \\
& \bar{T}^{H}=T_{0}^{H}-\Delta \theta_{0}\left|\frac{\partial \bar{T}}{\partial \theta_{0}}\right|_{\phi_{0}, \theta_{\beta}} \mid
\end{aligned}
$$

given that:

$$
\begin{aligned}
& \left.\frac{\partial \bar{L}}{\partial \theta_{0}}\right|_{\phi_{0}, \theta_{\beta}} \simeq 0 \\
& \left.\frac{\partial \bar{L}}{\partial \theta_{\beta}}\right|_{\phi_{0}, \theta_{0}}>0 \\
& \left.\frac{\partial \bar{T}}{\partial \theta_{0}}\right|_{\phi_{0}, \theta_{\beta}}<0 \\
& \left.\frac{\partial \bar{T}}{\partial \theta_{\beta}}\right|_{\phi_{0}, \theta_{0}} \simeq 0
\end{aligned}
$$

for $\pi / 4<\theta_{0}^{H}<\pi / 2, \theta_{\beta}^{H}=0^{\circ}$.

Vehicle yaw is actuated through differential changes in foil thrust, just as during cruising, however now it is actuated entirely through changes in foil twist amplitudes, $\theta_{0}$. For each foil:

$$
\theta_{0_{i}}=\theta_{0}-T_{i} k_{T}^{h}
$$

where the scalar $k_{T}^{h}>0$ is a heuristically tuned feedback gain and the subscript $i$ corresponds to the foil as in (3.10). Twist bias is perturbed from a baseline of $0^{\circ}$, just as for the cruising case, to control foil lift according to:

$$
\theta_{\text {bias }, i}=L_{i} k_{L}^{h}
$$

where the scalar $k_{L}^{h}$ is a heuritically tuned feedback gain. As a result, vehi- 
cle roll and pitch actuation are generated entirely through changes in $\theta_{\beta}$. Combining (3.10), (3.20), and (3.21) the desired foil kinematics for hovering are:

$$
\begin{aligned}
& f_{1-4}=f \\
& \phi_{0_{1-4}}=\phi_{0} \\
& \phi_{\beta_{1-4}}=0 \\
& \theta_{0_{1}}=\theta_{0}+k_{T}^{h}\left(-\frac{\tau_{3}}{4}+\frac{u_{1}}{4}\right) \quad \theta_{\beta_{1}}=k_{L}^{h}\left(\frac{\tau_{1}}{4}+\frac{\tau_{2}}{4}+\frac{u_{3}}{4}\right) \\
& \theta_{0_{2}}=\theta_{0}+k_{T}^{h}\left(\frac{\tau_{3}}{4}+\frac{u_{1}}{4}\right) \quad \theta_{\beta_{2}}=k_{L}^{h}\left(-\frac{\tau_{1}}{4}+\frac{\tau_{2}}{4}+\frac{u_{3}}{4}\right) \\
& \theta_{03}=\theta_{0}+k_{T}^{h}\left(\frac{\tau_{3}}{4}-\frac{u_{1}}{4}\right) \quad \theta_{\beta_{3}}=k_{L}^{h}\left(\frac{\tau_{1}}{4}-\frac{\tau_{2}}{4}+\frac{u_{3}}{4}\right) \\
& \theta_{0_{4}}=\theta_{0}+k_{T}^{h}\left(-\frac{\tau_{3}}{4}-\frac{u_{1}}{4}\right) \quad \phi_{\beta_{4}}=k_{L}^{h}\left(-\frac{\tau_{1}}{4}-\frac{\tau_{2}}{4}+\frac{u_{3}}{4}\right)
\end{aligned}
$$

The authority available to the foils in actuating all degrees of freedom can be changed simply by increasing either frequency or roll amplitude of all four foils. In practice, the minimum bound for $\left(\omega, \phi_{0}\right)$ is the least energetic stroke that is capable of righting the vehicle and overcoming positive buoyancy to drive the vehicle to depth. Furthermore, $\theta_{\beta}$ must not saturate solely to maintain depth: saturation on all foils renders the vehicle unable to apply any actuation to attitude control, and saturation on one or more foils causes unpredictable coupling between degrees of freedom as assumptions about symmetry and superposition are broken.

\subsubsection{Tuning and Initial Maneuvering Experiments Under Joystick Control}

During operation with the vehicle in a stable trim configuration, it was possible for the operator to control vehicle attitude, speed, depth and heading, using the joystick

system described in Chapter 1, with an open loop mapping of joystick positions to foil flapping parameters. When the vehicle was first changed to the unstable trim configuration, it immediately became clear that any successful joystick operation 


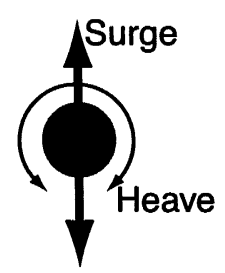

Left Joystick

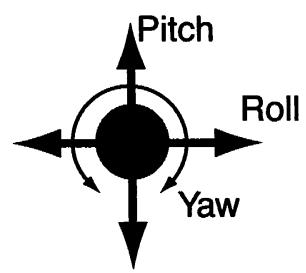

Right Joystick

Figure 3-11: Joystick Mapping

would need to incorporate automatic attitude regulation. Attitude instability required that the joystick inputs be defined in terms of the desired vehicle behavior rather than foil kinematics; and the burden of direct attitude regulation was removed from the user by overlaying automatic control of roll, pitch and depth on to the set points specified by manipulating the joysticks.

Figure 3-11 illustrates the general mapping between joysticks and vehicle behavior for both hovering operation and cruising operation. The right joystick controls the rotational degrees of freedom, the left joystick controls the linear degrees of freedom, and the rocker is a "throttle" controlling the frequency of the baseline flapping motion. The joystick positions are mapped either to actuation levels or to reference values. As shown in Figure 3-11, left-stick forward/back evokes thrust from all four foils; right-stick forward/back sets desired pitch angle; right-stick left/right sets desired roll angle; right stick twist generates a yaw moment. Less intuitively, left-stick twist controls heave set point.

Initial large angle maneuvering experiments were performed under joystick control in order to ease troubleshooting of both navigation and control systems. Using the joystick allowed extended runs in the pool for initial tuning of control loops by removing any concerns about position errors in navigation and wall collision, and provided an immediate intuitive grasp of the vehicle capabilities and foil dynamic range. Through trial and error, it was found that baseline kinematics defined by a flapping frequency of $1.25 \mathrm{~Hz}$, roll amplitude of $30^{\circ}$, and twist amplitude of $65^{\circ}$ provided effective and responsive hovering operation. For a typical test, the vehicle righted itself at the surface by either rolling or pitching $180^{\circ}$, and descended to the 


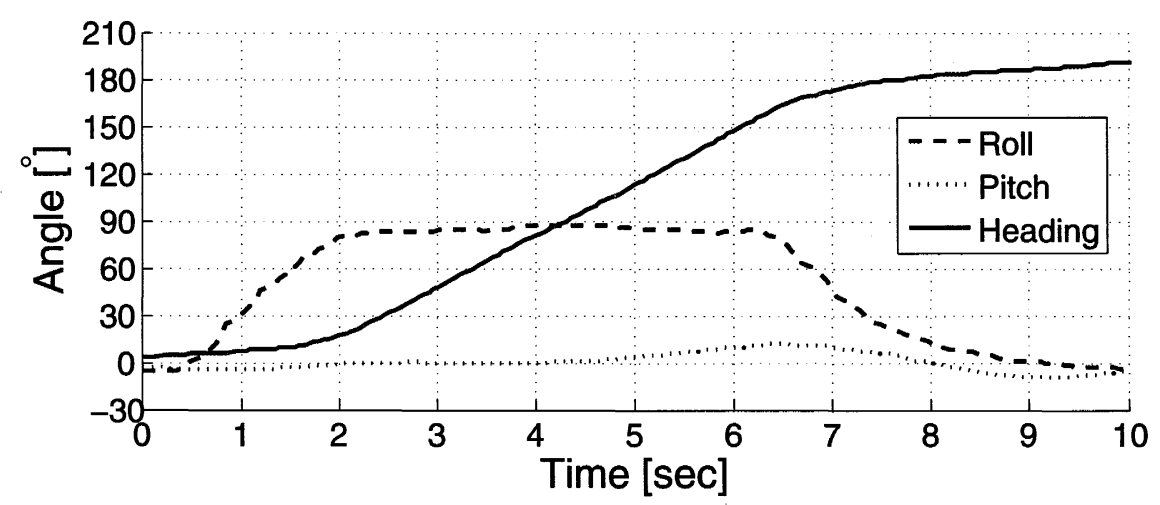

Figure 3-12: Vehicle attitude during joystick controlled banked turn.

desired depth. The vehicle was yawed about to the desired start direction for the test, and then switched into cruising control mode via a switch on the joystick box. During cruising, the baseline foil kinematics were held at a flapping frequency of $1 \mathrm{~Hz}$, roll amplitude of $35^{\circ}$, and twist amplitude of $45^{\circ}$. The twist amplitude is reduced from $65^{\circ}$ to $45^{\circ}$ in cruising to increase the baseline thrust from all actuators, as they are no longer acting in opposition to one another but rather working together to drive the vehicle forward. The combination of parameters, particularly the choice of $\theta_{0}$, was a compromise between two competing requirements; maximum forward speed and the acceleration from from rest. Halting of forward speed and resumption of hovering was accomplished by flipping the switch on the joystick box back to the hovering position.

Once the control gains were properly tuned, the vehicle was successfully steered in approximately $180^{\circ}$ heading changes both while level, and while rolled by $90^{\circ}$ into the turn. Vehicle attitude for two reprsentative trials are plotted in Figures 3-12 and Figure 3-13. Figure 3-12 shows the results from a banked turn (as captured in a frame from underwater video camear in Figure 3-1). The vehicle is first rolled to $90^{\circ}$ in 1.5 seconds, after which the turn is initiated. The vehicle holds the roll angle throughout the turn, and at 6.5 seconds is commanded back to level. Maximum rate of heading 


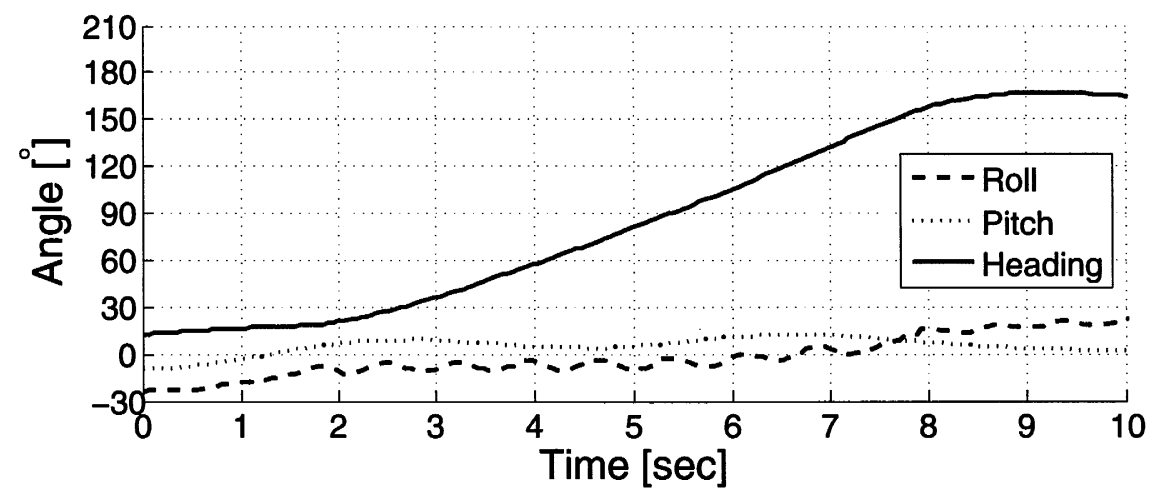

Figure 3-13: Vehicle attitude during joystick controlled level turn.

change is $34^{\circ} / \mathrm{s}$, and a $180^{\circ}$ heading change is achieved in less than 5.5 seconds, if entry and exit roll time is not included. With entry and exit roll time, the turn takes approximately 7.5 seconds.

Figure 3-13 shows the results of the level turn. The roll angle oscillates with a peak to peak excursion of approximately $7^{\circ}$ during the turn, with a frequency of $1.25 \mathrm{~Hz}$, which matches the flapping frequency of the foils. This rocking motion is a symptom of the larger roll amplitude of the foils on the outside of the turn, which powers the turn but also results in larger amplitude lift oscillation from the outside foils. Maximum rate of heading change is $23^{\circ} / \mathrm{s}$, and a 150 degree heading change is achieved in approximately 6 seconds.

\subsection{Maneuvering Experiments}

A commonly reported value for both AUV and animal maneuvering performance is the turning radius, typically as a function of, or as a single data point with, the turn entry speed. For comparison across widely varying length scales, turning radius is reported as normalized by body length (BL). By this metric, the maneuvering abilities 


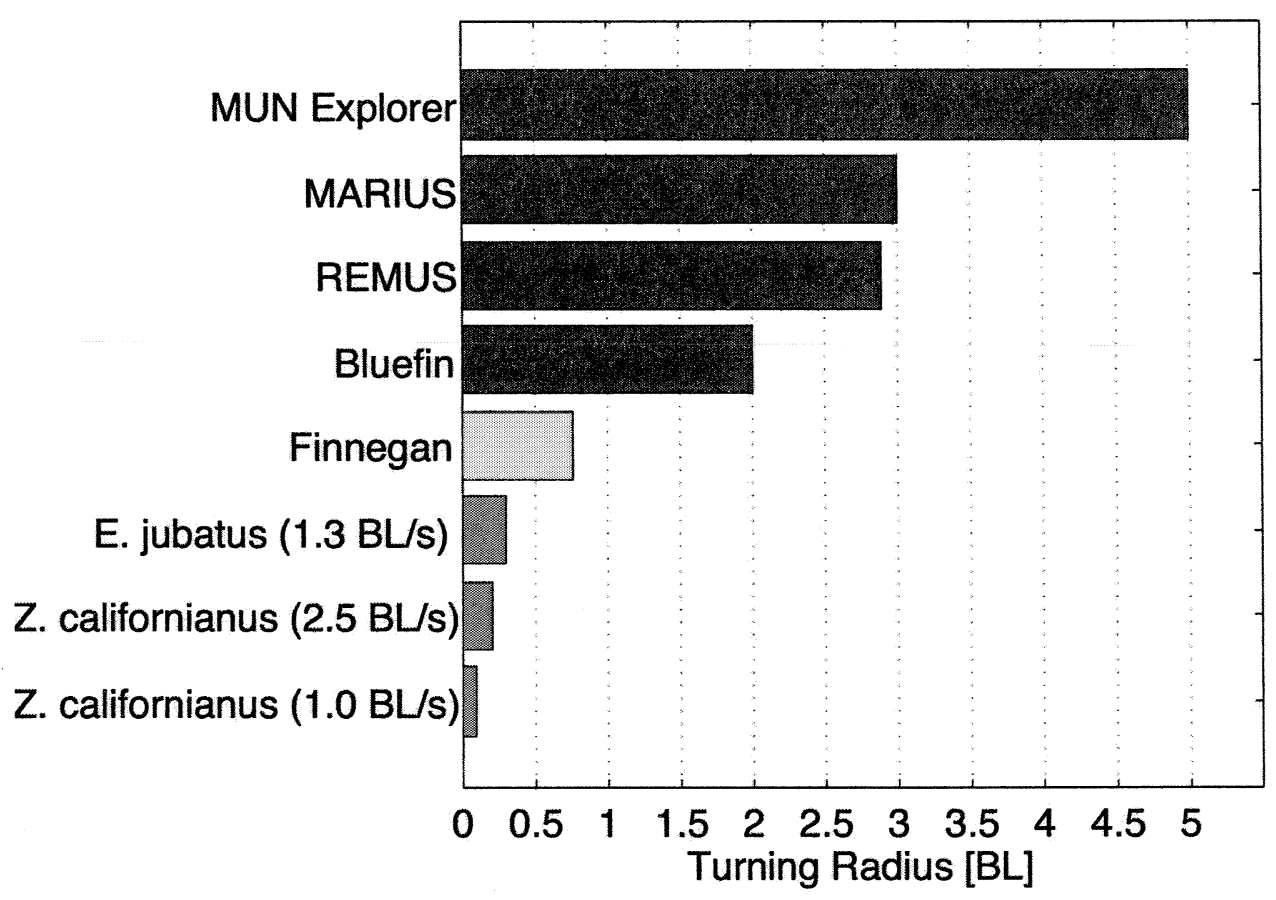

Figure 3-14: Finnegan best turning performance at speed as compared to turning radii of vehicles with various conventional thruster/control surface combinations, as well as sealion (Zalophus californianus and Eumatopias jubatus) turning rates (see Table 3.1 for values and sources).

of marine animals exceed those of the nimblest AUVs by an order of magnitude. The reported values for a number of AUVs of varying length scales, with a variety of traditional thruster configurations, are shown in Table 3.1, and graphically compared for emphasis in Figure 3-14. Note that the turning radii radii for sealions, (Zalophus californianus and Eumatopias jubatus ) taken from Fish [18] and Cheneval [9] are given for speeds of 1 and $2 \mathrm{BL} / \mathrm{s}$, in comparison to the $0.5 \mathrm{BL} / \mathrm{s}$ typically reported for AUVs. The turning radius of $0.77 \mathrm{BL} @ 0.72 \mathrm{BL} / \mathrm{s}$ for Finnegan is the best value recorded for a $180^{\circ}$ turn during the testing described in the following sections.

Three basic maneuvers were chosen for extensive testing to determine the effect of foil actuation on AUV maneuvering performance: level turns, banked turns, and "twisting" turns. All three turns were used to generate $90^{\circ}$ heading changes starting from a level cruising state. The banked turn was also used to generate $180^{\circ}$ and $360^{\circ}$ turns from a level cruise. No attempt was made to formally optimize the turning

\footnotetext{
${ }^{1}$ Personal communication, T. Stefanov-Wagner, 21 November 2007
} 


\begin{tabular}{|c|c|c|}
\hline Vehicle & Turning & Thruster Configuration \\
Name & Radius [BL] & \\
\hline MUN Explorer [32] & 5 @ $0.25 \mathrm{BL} / \mathrm{s}$ & 1 rear mounted thruster, 6 planes \\
MARIUS [52] & 3 @ 0.5 & 2 forward thrusters, 4 tunnel thrusters, 3 planes \\
REMUS [1] & $2.9 @ 0.5$ & 2 forward thrusters, 2 pitch and 2 yaw planes \\
Bluefin-21 & 2 @ 0.5 & 1 2-DOF rearmounted azimuthing thruster \\
Finnegan & $0.77 @ 0.72$ & 4 oscillating foil actuators \\
\hline E. jubatus [9] & $0.3 @ 1.3 \mathrm{BL} / \mathrm{s}$ & \\
Z. californianus [18] & $0.22 @ 2 \mathrm{BL} /$ & \\
$"$ & $0.10 @ 1 \mathrm{BL} / \mathrm{s}$ & \\
\hline
\end{tabular}

Table 3.1: Reported turning radius of AUVs with comparison to the sealions Zalophus californianus and Eumatopias jubatus

performance. The sheer number of significant parameters involved in the turning maneuvers rendered any true optimization too costly for pool testing. The vehicle maneuvering performance was tested in the same pool described in the previous chapter, the the $25 \mathrm{~m}$ x $15 \mathrm{~m}$ MIT Alumni Swimming Pool. All tests were initiated at the surface with attitude inverted, as a result of the passively unstable trim condition and slight positive buoyancy. The vehicle was tethered throughout testing, both as a safety precaution to prevent wall collisions and to allow communication before, during and after individual experiments.

Joystick vehicle control was useful for tuning control loops, demonstrations and initial investigation of maneuvers. However, scripted mission following allowed for repeatable experimentation to determine vehicle performance in large angle maneuvers, through automatically timed sequences of precise attitude setpoints. In addition to allowing for greater accuracy in timing and setpoint values, scripted mission following allowed greater freedom to change the baseline foil kinematics during a maneuver. Different kinematics are naturally more effective for different forward swimming tasks, such as acceleration, high speed cruising and aggressive turning, but adding control of multiple baseline kinematic parameters would likely overwhelm a single operator already charged with controlling vehicle attitude, heading and depth, regardless of the limitations of the joystick system. 


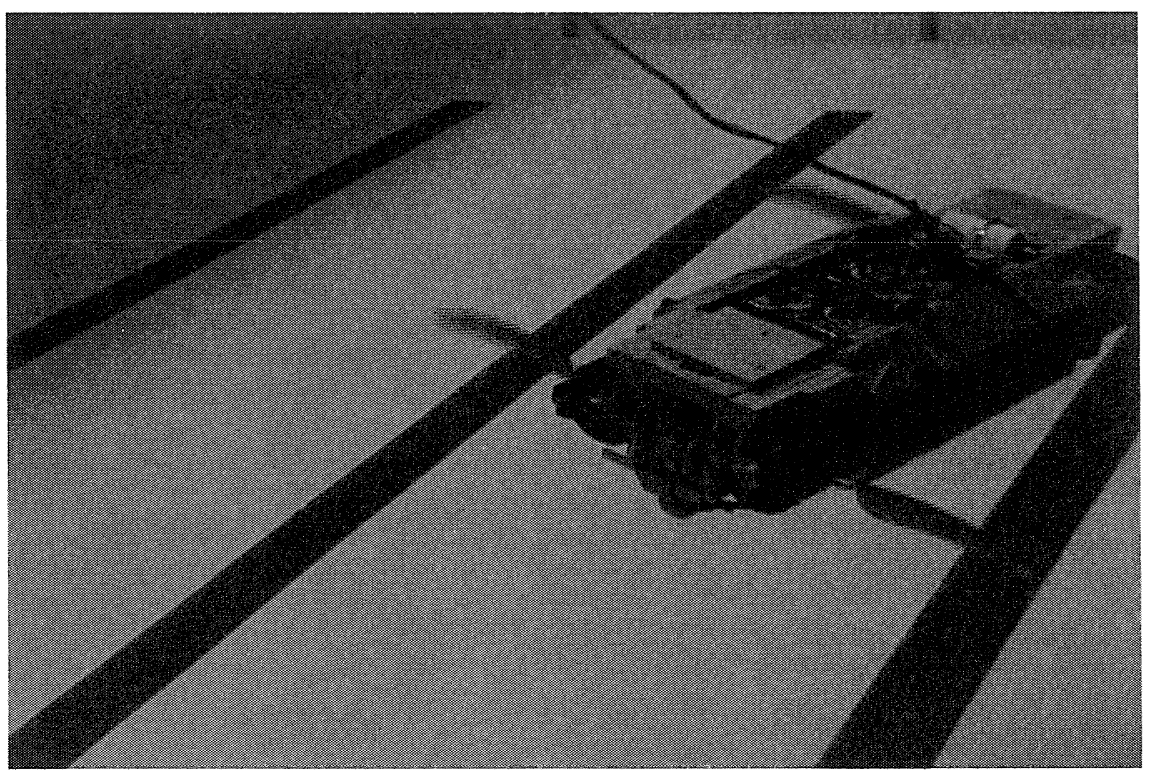

Figure 3-15: Screen capture from underwater video footage showing vehicle during test at the MIT Alumni Pool. As shown, the vehicle is in the unstable trim configuration, with no fairing.

\subsubsection{Level Turning}

The first type of turn tested, the level turn, was the least effective both for rapid heading change and aggressive obstacle avoidance. In a level turn, the vehicle maintains zero roll and zero pitch reference, while changing heading through body fixed yaw actuation. A level turn with an initial forward speed of $0.72 \mathrm{~m} / \mathrm{s}[0.55 \mathrm{BL} / \mathrm{s}]$, and baseline foil kinematics defined by $f=1 \mathrm{~Hz}, \phi_{\max }=30^{\circ}, \theta_{\max }=45^{\circ}$ (resulting $S t=0.72, \alpha_{\max }=26^{\circ}$ ) is illustrated with vehicle position and orientation shown at 1 second intervals, from two different views in Figures 3-17 and 3-18. This turn is denoted as case $\mathbf{A}$ in Table 3.2. The attitude and position are plotted for this turn as a function of time in Figure 3-19, and the velocity and angular rates in Figure 3-20. The vehicle velocity is perpendicular to the initial velocity within 8.75 seconds of the initiation of the turn, having travelled 5 meters in the initial vehicle direction, and exits the turn with forward velocity of $0.75 \mathrm{~m} / \mathrm{s}[0.58 \mathrm{BL} / \mathrm{s}]$. Maximum heading rate during the turn is $27^{\circ} / \mathrm{s}$. 


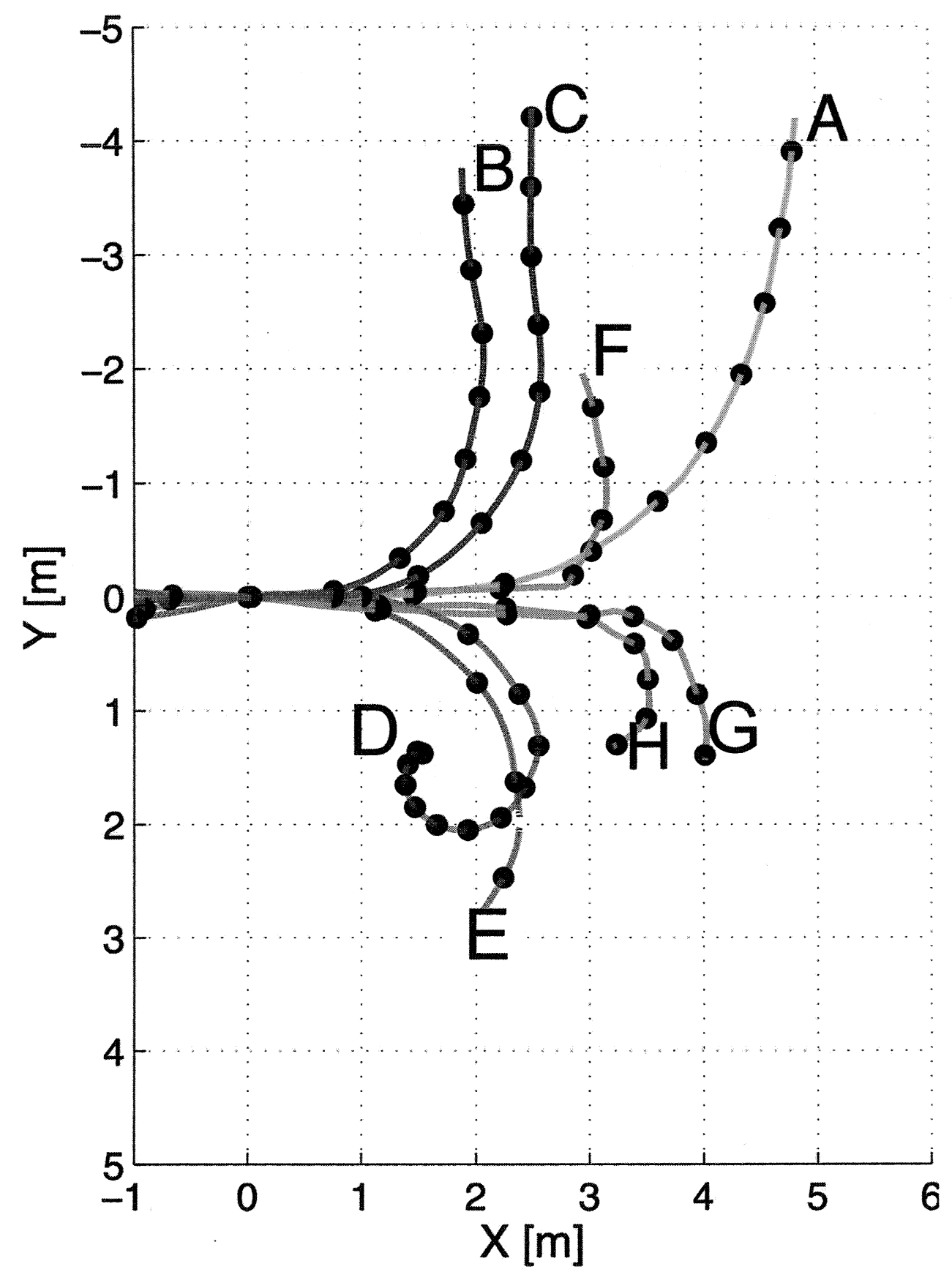

Figure 3-16: Top view of tracklines for all turns discussed above. All tracklines are translated and rotated such that the track passes through the origin at the point where the turn is initiated, with velocity in the $+\mathrm{X}$ direction. The level turn $(\mathbf{A})$ is in green, banked turns $(\mathbf{B}, \mathbf{C}, \mathbf{D}, \mathbf{E})$ are in blue, and twisting turns $(\mathbf{F}, \mathbf{G}, \mathbf{H})$ in red. 


\begin{tabular}{|c|c|c|c|c|c|c|c|c|c|c|c|c|}
\hline & & \multicolumn{5}{|c|}{ Entry Kinematics } & \multicolumn{4}{|c|}{ Turn Kinematics } & \multicolumn{2}{|c|}{ Results } \\
\hline & & $\begin{array}{c}f \\
{[\mathrm{~Hz}]} \\
\end{array}$ & $\begin{array}{l}\phi_{0} \\
{[\circ]} \\
\end{array}$ & $\begin{array}{l}\theta_{0} \\
{\left[{ }^{\circ}\right]} \\
\end{array}$ & $S t$ & $\begin{array}{c}\alpha_{\max } \\
\left.{ }^{\circ}\right] \\
\end{array}$ & $\begin{array}{c}f \\
{[\mathrm{~Hz}]} \\
\end{array}$ & $\begin{array}{l}\phi_{0} \\
{\left[{ }^{\circ}\right]}\end{array}$ & $\begin{array}{l}\theta_{0} \\
{\left[{ }^{\circ}\right]}\end{array}$ & $\begin{array}{c}\theta_{\beta}^{S A T} \\
{\left[{ }^{\circ}\right]} \\
\end{array}$ & $\begin{array}{l}\text { Entry } \\
\text { Speed } \\
{[\mathrm{BL} / \mathrm{s}]} \\
\end{array}$ & $\begin{array}{c}\text { Obstacle } \\
\text { Avoidance } \\
\text { BL }\end{array}$ \\
\hline Level & $\mathbf{A}$ & $\overline{11}$ & 30 & 45 & 0.72 & 26 & 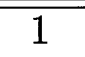 & 30 & 45 & - & 0.55 & 3.8 \\
\hline Banked & $\begin{array}{l}\text { B } \\
\text { C } \\
\text { D } \\
\text { E }\end{array}$ & $\begin{array}{c}1 \\
1 \\
0.75 \\
1\end{array}$ & $\begin{array}{l}30 \\
30 \\
60 \\
60\end{array}$ & $\begin{array}{l}30 \\
30 \\
45 \\
45\end{array}$ & $\begin{array}{c}0.67 \\
0.64 \\
0.8 \\
1.1\end{array}$ & $\begin{array}{l}35 \\
34 \\
31 \\
37\end{array}$ & $\begin{array}{l}0.5 \\
" \\
" \\
"\end{array}$ & $\begin{array}{l}60 \\
" \\
" \\
"\end{array}$ & $\begin{array}{l}45 \\
" \\
" \\
"\end{array}$ & $\begin{array}{l}45 \\
25 \\
45 \\
45\end{array}$ & $\begin{array}{l}0.54 \\
0.55 \\
0.72 \\
0.75\end{array}$ & $\begin{array}{c}1.5 \\
1.9 \\
1.9(0.8)^{*} \\
1.8\end{array}$ \\
\hline Twisting & $\begin{array}{l}\mathbf{F} \\
\mathbf{G} \\
\mathbf{H}\end{array}$ & $\begin{array}{l}1 \\
1 \\
1\end{array}$ & $\begin{array}{l}30 \\
60 \\
60\end{array}$ & $\begin{array}{l}30 \\
45 \\
45\end{array}$ & $\begin{array}{c}0.64 \\
1.1 \\
1.1\end{array}$ & $\begin{array}{l}34 \\
37 \\
37\end{array}$ & " & $\begin{array}{l}" \\
"\end{array}$ & $\begin{array}{l}" \\
" \\
"\end{array}$ & $\begin{array}{l}45 \\
25 \\
45\end{array}$ & $\begin{array}{l}0.55 \\
0.75 \\
0.75\end{array}$ & $\begin{array}{l}2.5 \\
3.2 \\
2.7\end{array}$ \\
\hline
\end{tabular}

Table 3.2: Baseline Kinematics for Turns Depicted in Figure 3-16. *Value in parenthesis is turning radius based on $180^{\circ}$ turn, for comparison to reported AUV operational turning radii.

Figure 3-21 shows the pitch and roll trajectories of all four foils during the turn, along with the estimated nominal angles of attack at the foil 0.7 span. The controller gains were such that the roll amplitude of the foils on the outside of the turn $(3,4)$ reached $\phi_{\max }=50^{\circ}$, while the the roll amplitude of the foils to the inside of the turn $(1,2)$ was reduced to as little as $\phi_{\max }=15^{\circ}$. Note that the angle of attack estimates for the inside foils are generally higher than those of the outside foils, despite the higher roll amplitude of the outside foils; the rigid body rotation increases the incoming flow velocity experienced by the outside foils, and decreases that experienced by the inside foils, changing the nominal angle of attack profiles accordingly.

Two details of the turn are immediately apparent. First, the vehicle sideslips significantly during the turn, reaching a body fixed sway velocity of $0.37 \mathrm{~m} / \mathrm{s}[0.28$ $\mathrm{BL} / \mathrm{s}]$, comparable to the entry velocity of $0.72 \mathrm{~m} / \mathrm{s}[0.55 \mathrm{BL} / \mathrm{s}]$. The sideslip increases the obstacle avoidance distance, as measured from the turn entry to the point where the vehicle velocity is perpendicular to the entry velocity. Note that with sideslip, the vehicle heading may be perpendicular to the initial heading before the vehicle velocity is perpendicular to the entry velocity. Indeed, from Figure 3-19, a heading change of $180^{\circ}$ is reached within 8 seconds, but the motion in the $\mathrm{X}$ direction (initial surge direction) is not halted until 8.75 secs in to the turn. 


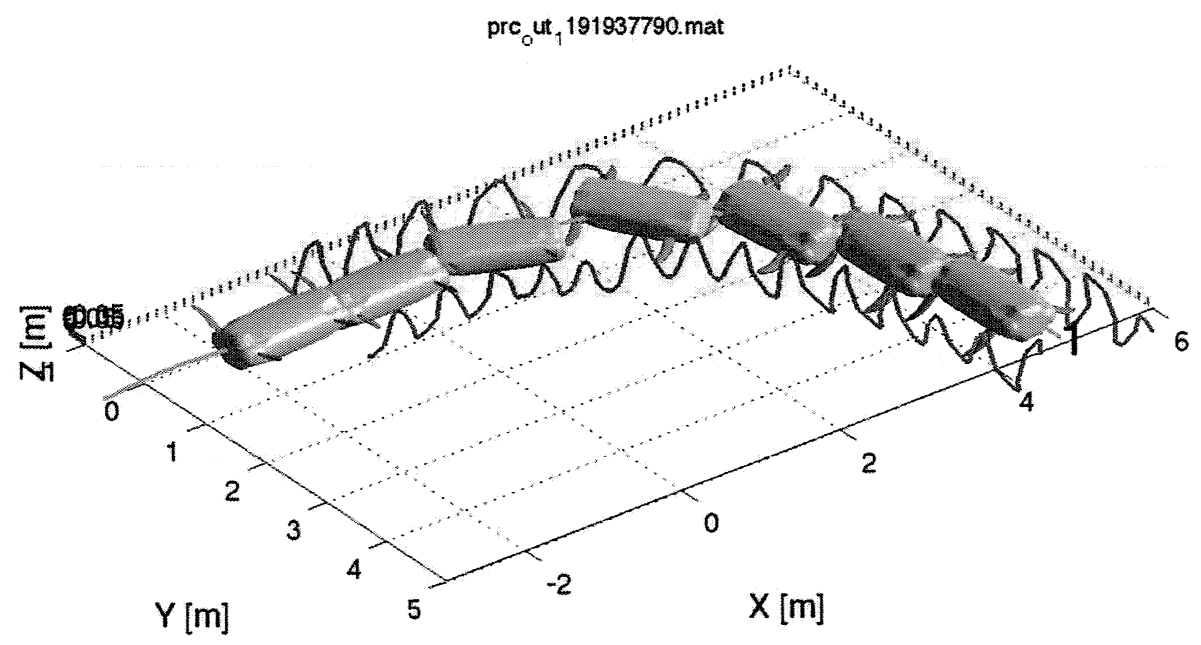

Figure 3-17: Visualization of a level turn. (Case A) Vehicle and foil positions and orientations are shown at one second intervals, with lines tracing the path of the foil tips through space.

Second, the vehicle roll angle oscillates with an amplitude of approximately $15^{\circ}$ (and a maximum roll rate of $75^{\circ} / \mathrm{s}$ ) during the first part of the turn where large yaw torque is demanded. Larger amplitude motion with the same frequency and twist amplitude on the outside foil results in larger amplitude oscillating lift force from the outside foils than from the inside foils, resulting in unbalanced roll torque. The period of the vehicle oscillation is thus identical to the period of the foil motion. It may be possible to restore the torque balance by decreasing the feathering of the inside foils, but this was not attempted and it is not clear whether this would reduce the turning speed by increasing the thrust generated by the inside foils.

Figure 3-16 shows a top view of the tracklines of all turns described in detail in this section. Compare curve A corresponding to this case with curves B-E to see the difference in turning performance between the level turn and several banked and twisting turns with similar baseline kinematics. It is immediately clear that the level 


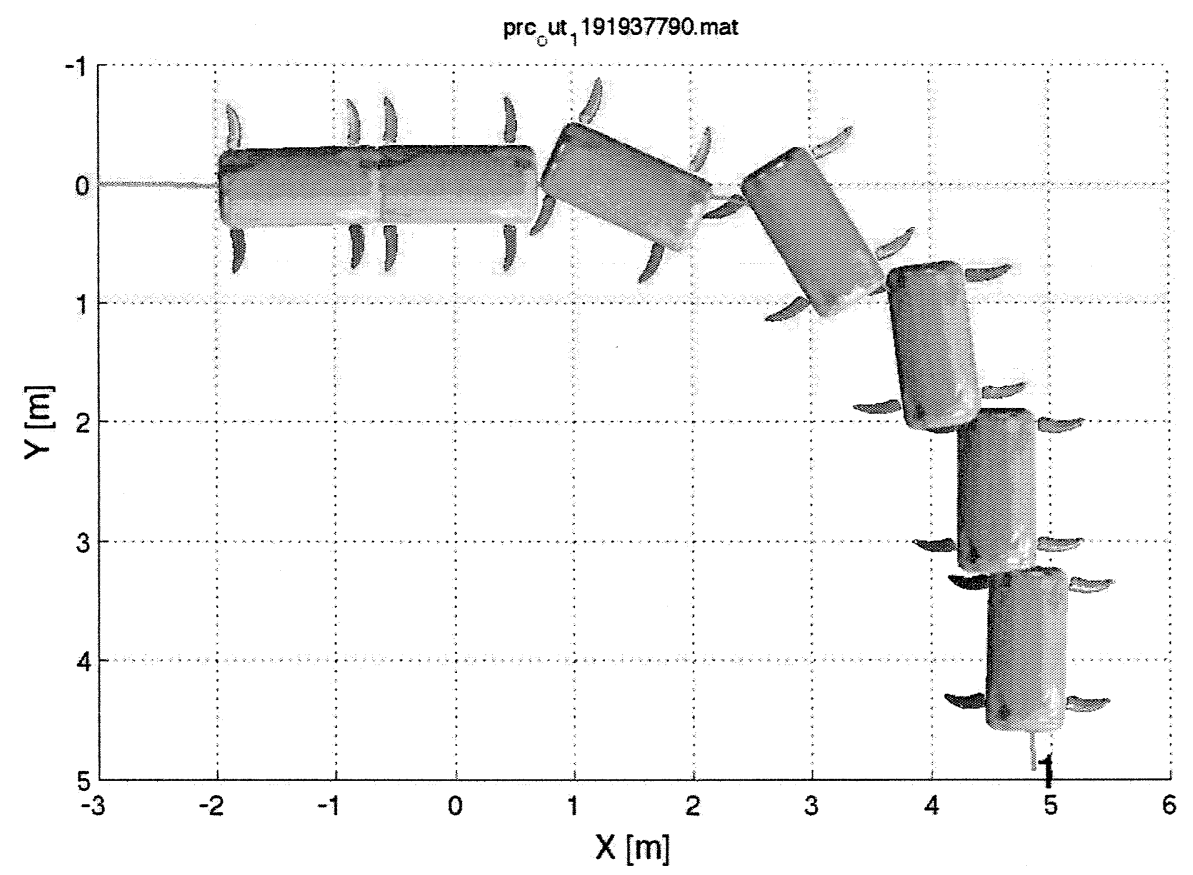

Figure 3-18: Top view of a level turn. (Case A) Vehicle and foil positions and orientations are shown at one second intervals.

turn takes significantly more time and space before the vehicle achieves a velocity perpendicular to the initial cruising direction. If we define the obstacle avoidance distance as the maximum excursion in the initial direction of travel from the point at which the turn was initiated, the obstacle avoidance distance of $5 \mathrm{~m}$ for the level turn is more than twice that of the best of the turns described below.

\subsubsection{Banked Turns}

During the banked turn, the vehicle changes heading by rotating about the body pitch axis, rather than the body yaw axis, as it does during the level turn. To make the turn without depth change, the vehicle must first be rolled $90^{\circ}$ to one side. For the sake of calculating obstacle avoidance distance, the beginning of the turn can be reasonably defined as either the beginning of the roll actuation needed to bring the vehicle to a $90^{\circ}$ roll angle, or the beginning of the yaw actuation which initiates the heading change. The second definition was chosen on the assumption that, without 

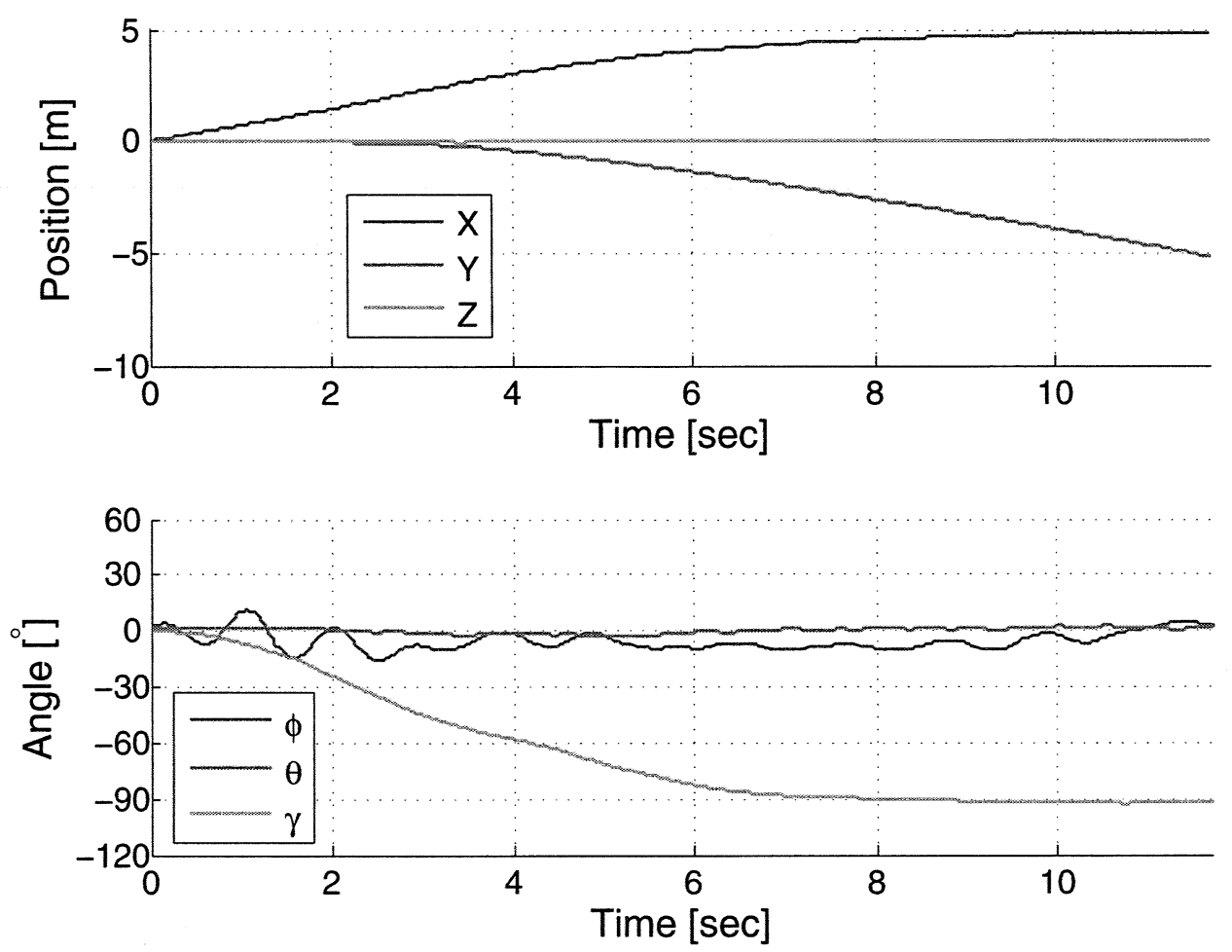

Figure 3-19: Vehicle position and attitude throughout level turn. (Case A)

the depth constraint imposed by the pool, a rapid pitch change without the initial rolling motion could be used as easily for initial evasion.

\section{Kinematics for Selected Banked Turns}

The tracklines for four different banked turns with two different entry speeds are included as Cases $\mathbf{B}, \mathbf{C}, \mathbf{D}$, and $\mathbf{E}$ in Figure 3-16. The tracklines cross the origin at $t=0$ according to the definition above, when the vehicle first begins attempting to actuate the heading change. All turns were performed to port to eliminate the effect of unknown asymmetries in vehicle mass distribution or geometry. For clarity, however, turns with a nominal entry speed of $0.5 \mathrm{BL} / \mathrm{s}$ are shown turning to port, while turns with a nominal entry speed of $0.75 \mathrm{BL} / \mathrm{s}$ are shown turning to starboard. (Entry speed was repeatable to within $0.05 \mathrm{BL} / \mathrm{s}$ from experiment to experiment 

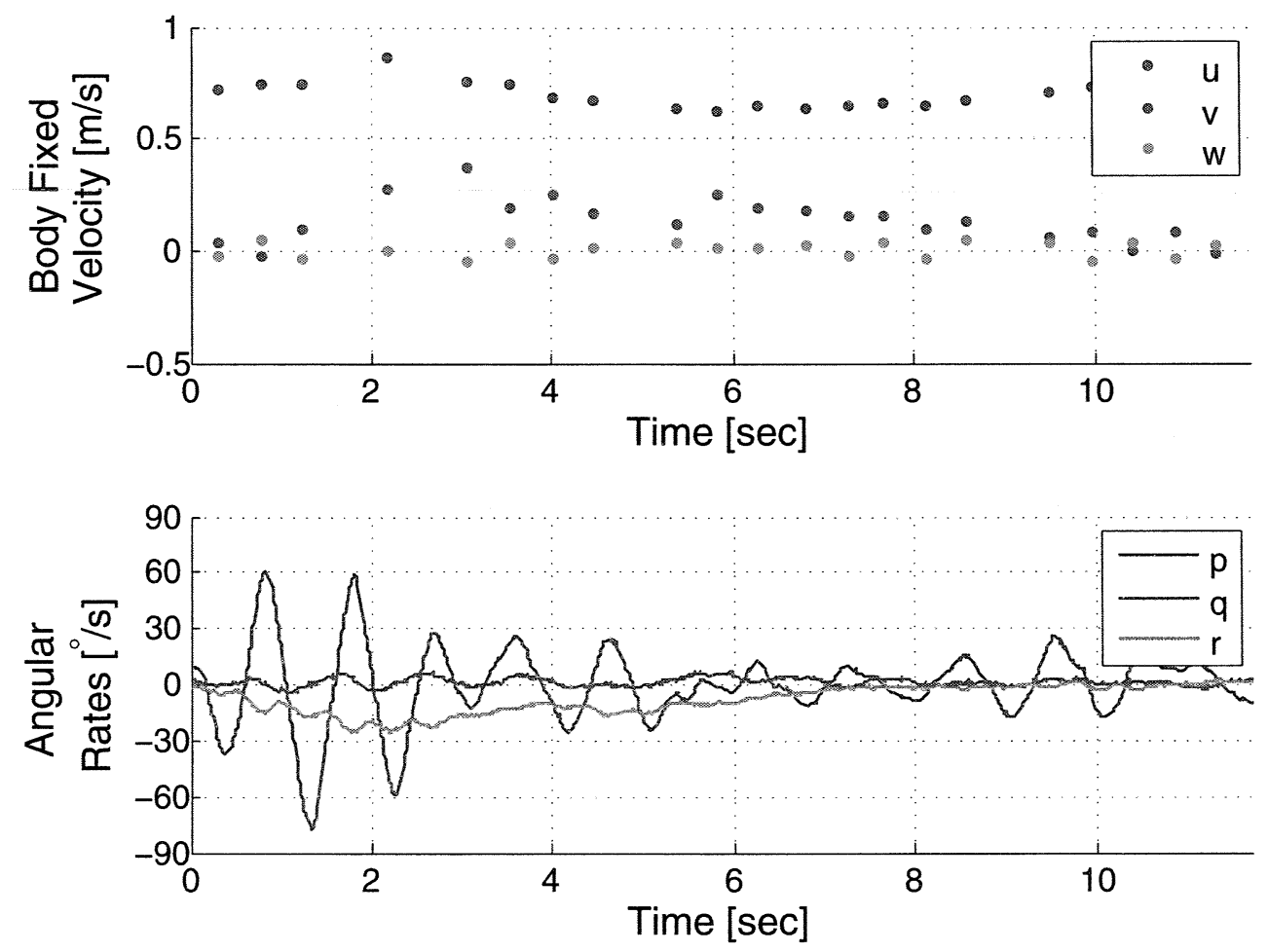

Figure 3-20: Vehicle velocities and angular rates throughout level turn. (Case A)

with the same kinematics.)

The kinematics used during the rolling phases and during the pitching phase of the four banked turns are given in Table 3.2. The initial velocities for Cases $\mathbf{B}$ and $\mathbf{C}$ are $0.78 \mathrm{~m} / \mathrm{s}[0.54 \mathrm{BL} / \mathrm{s}]$ and $0.82 \mathrm{~m} / \mathrm{s}[0.55 \mathrm{BL} / \mathrm{s}]$. The baseline kinematics are identical $\left(f=0.5 \mathrm{~Hz}, \phi_{0}=60^{\circ}, \theta_{0}=40^{\circ}\right.$ during the pitching phase; $f=1.0 \mathrm{~Hz}, \phi_{0}=30^{\circ}, \theta_{0}=$ $30^{\circ}$ before the turn and during rolling phases) resulting in $\left(S t, \alpha_{\max }\right)=\left(0.67,35^{\circ}\right)$ for B and $\left(S t, \alpha_{\max }\right)=\left(0.64,34^{\circ}\right)$ for $\mathbf{C}$ when the vehicle enters the turn. The two cases differ only in the saturation limits imposed for foil twist bias for pitch actuation, with $\theta_{\beta}^{S A T}=45^{\circ}$ for $\mathbf{B}$ and $25^{\circ}$ for $\mathbf{C}$.

The the initial velocities for Cases $\mathbf{D}$ and $\mathbf{E}$ are $0.93 \mathrm{~m} / \mathrm{s}[0.72 \mathrm{BL} / \mathrm{s}]$ and 0.98 $\mathrm{m} / \mathrm{s}[0.75 \mathrm{BL} / \mathrm{s}]$, respectively. Baseline kinematics before the turn and during the rolling phases are more aggressive than for $\mathbf{B}$ and $\mathbf{C}(f=0.75 \mathrm{~Hz}$ for $\mathbf{D}, f=1.0 \mathrm{~Hz}$ 


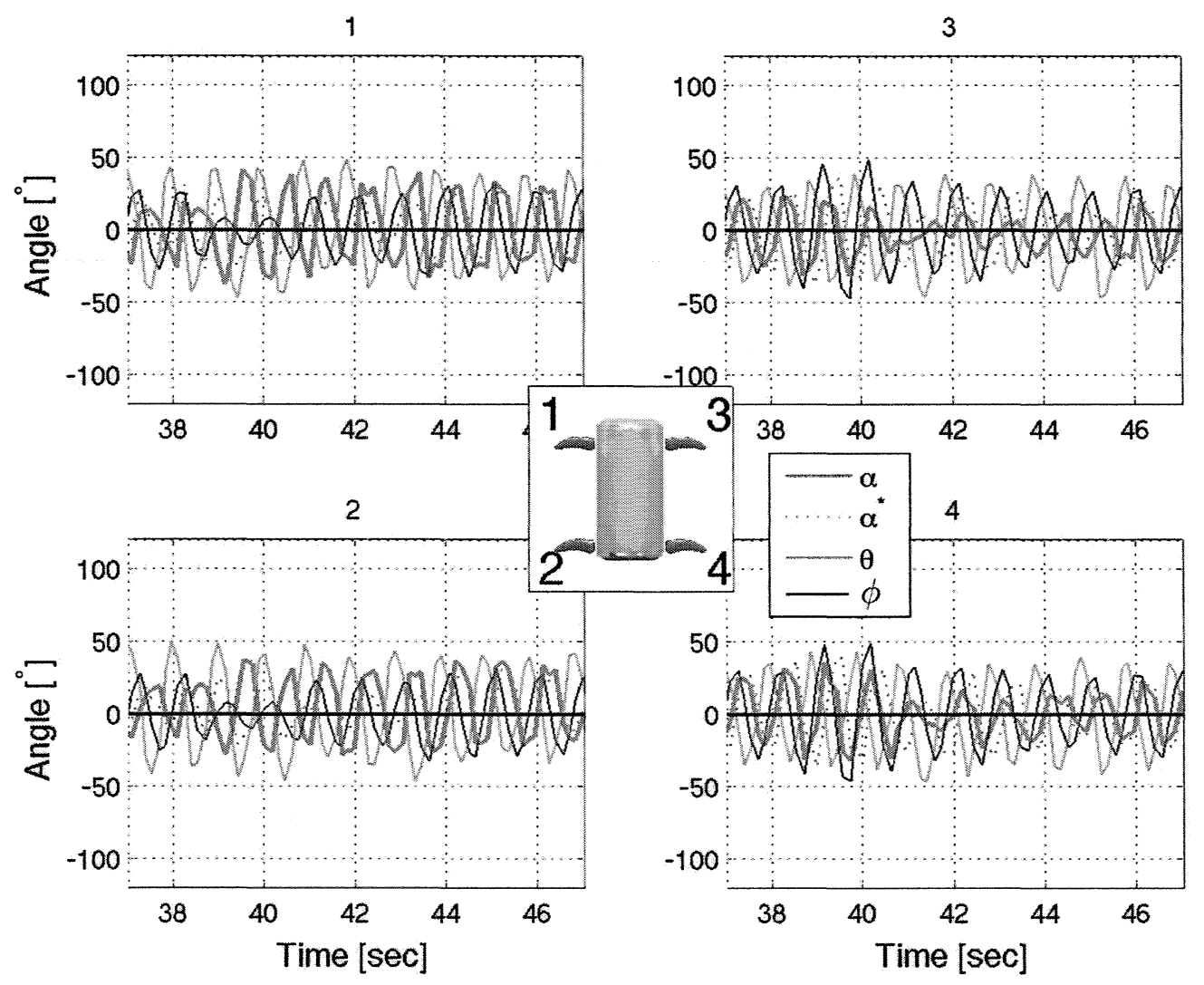

Figure 3-21: Roll angle $\phi(t)$ (blue), pitch angle $\theta(t)$ (green), and nominal angle of attack $\alpha(t)$ (red) resulting from foil motion, vehicle velocity, and vehicle rotation, throughout level turn. (Case $\mathbf{A}$ ).

for $\left.\mathbf{E} \phi_{0}=60^{\circ}, \theta_{0}=45^{\circ}\right)$ resulting in $\left(S t, \alpha_{\max }\right)=\left(0.8,31^{\circ}\right)$ for $\mathbf{D}$ and $\left(S t, \alpha_{\max }\right)=$ $\left(1.1,37^{\circ}\right)$ for $\mathbf{D}$. The baseline kinematics during the pitching phase, however, are identical across all four cases. Case $\mathbf{D}$ and $\mathbf{E}$ differ also in that for Case $\mathbf{D}$ the heading reference is set to $360^{\circ}$ to test the minimum vehicle turning radius.

\section{Comparison and Discussion of Results from Selected Banked Turns}

Case B, as shown by the trackline in Figure 3-16 has a forward obstacle avoidance distance of just $2.0 \mathrm{~m}[1.54 \mathrm{BL}]$ the shortest recorded across all tests. Case $\mathbf{B}$ is depicted in Figures 3-22 and 3-23, with the vehicle position and orientation shown from two different views at 1 second intervals. The attitude and position are plotted for this turn as a function of time in Figure 3-24, showing that the initial rolling 


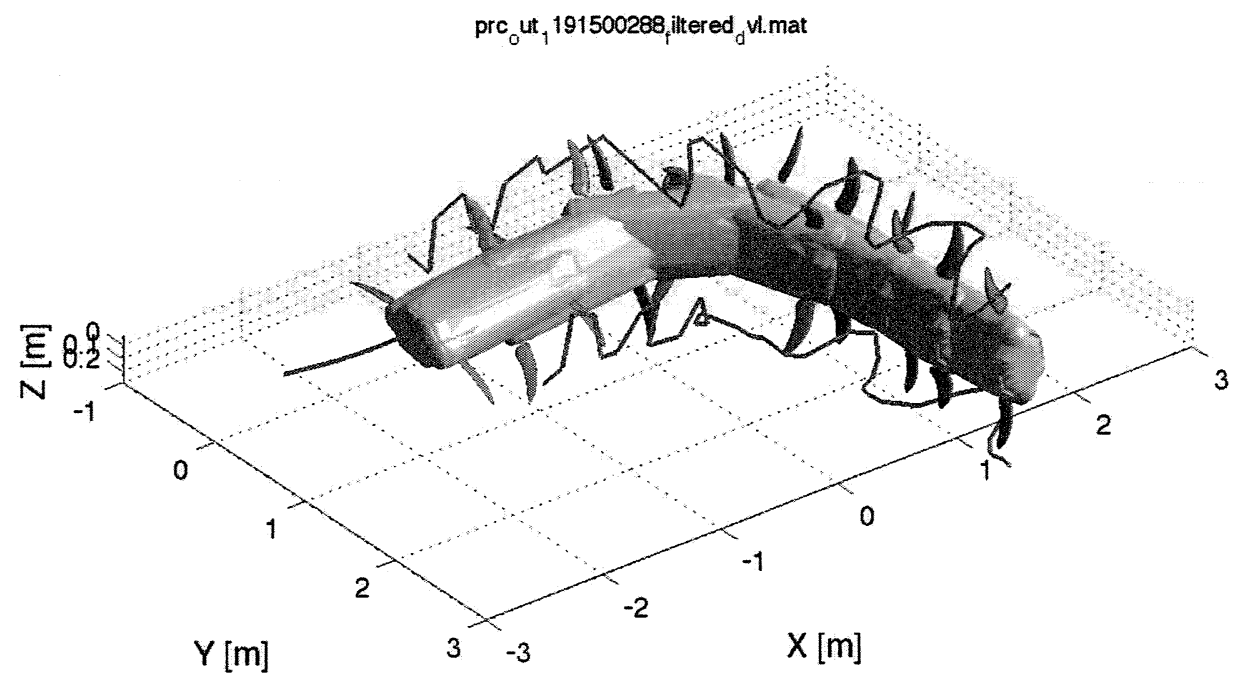

Figure 3-22: Visualization of a banked turn. (Case B) Vehicle and foil positions and orientations are shown at one second intervals, with lines tracing the path of the foil tips through space.

motion in this case is accomplished in approximately 1 second, over the course a single full stroke, with a maximum roll rate of $142^{\circ} / s$ recorded. The vehicle heading reference is changed from $0^{\circ}$ to $-90^{\circ}$ when the vehicle roll exceeds $60^{\circ}$ degrees, and the vehicle achieves a heading change of $85^{\circ}$ in less than 4 seconds by rapidly pitching with a maximum resulting heading rate of $40^{\circ} / \mathrm{s}$. As noted above, the forward obstacle avoidance is less than half of that of the level turn of case $\mathbf{A}$.

The control gains and saturation limits imposed were such that the saturation limit in pitch actuation was reached at the start of every banked turn tested. The resulting sensitivity to the saturation limit in pitch can be seen through comparison of curves $\mathbf{C}$ and $\mathbf{B}$ in Figure 3-16. With the higher saturation limit for $\mathbf{B}$, the initial heading rate is significantly faster, but this faster heading change is accompanied by a greater drop in speed during the turn, because the foil thrust is reduced as the pitch bias is increased, as predicted from Figure 3-9. 


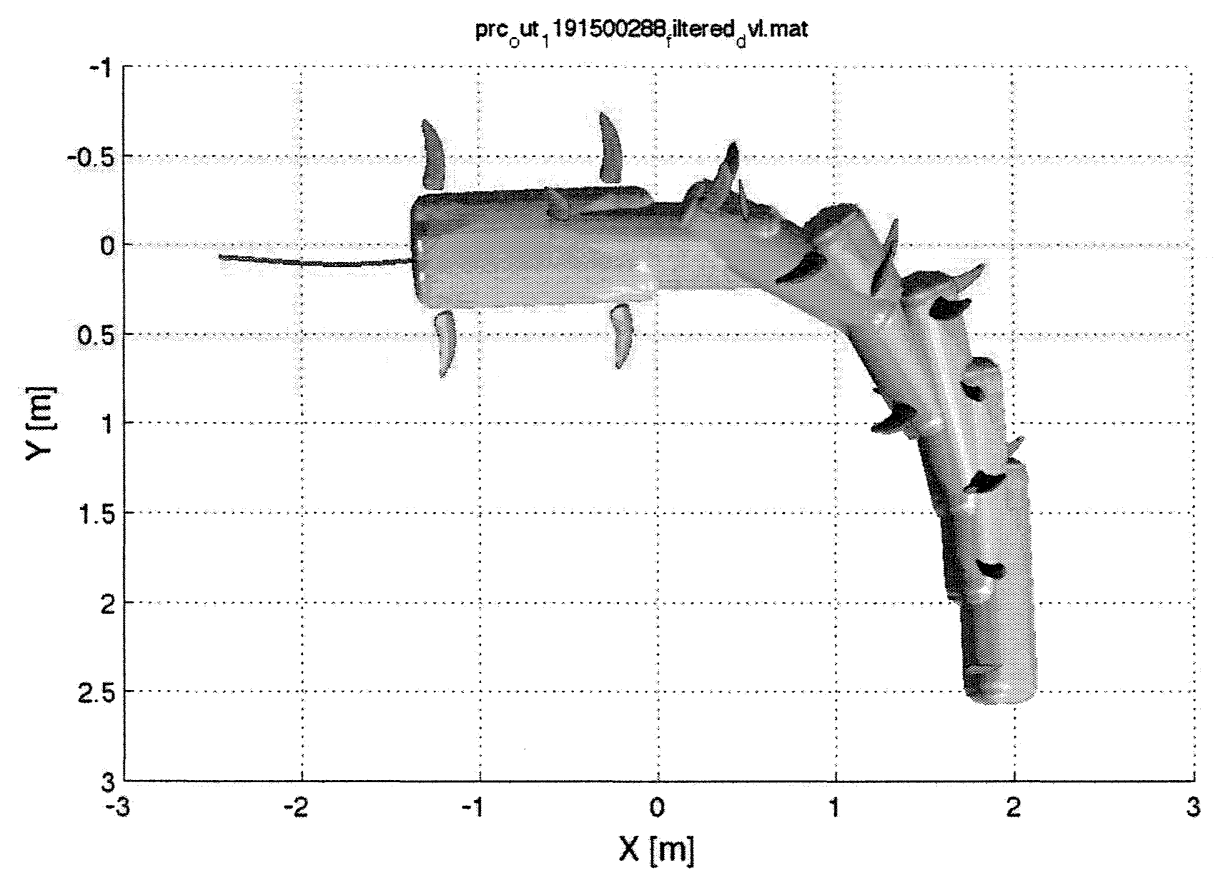

Figure 3-23: Top view of a banked turn. (Case B) Vehicle and foil positions and orientations are shown at one second intervals.

Curve $\mathbf{E}$ in Figure 3-16 shows the trackline of a turn with an initial velocity of $0.98 \mathrm{~m} / \mathrm{s}[0.75 \mathrm{BL} / \mathrm{s}]$, and an obstacle avoidance distance of $2.4 \mathrm{~m}$ [1.85 BL], which is the shortest recorded for tests with a nominal entry speed of $0.75 \mathrm{BL} / \mathrm{s}$. Even with the higher velocity, the obstacle avoidance distance is less than half that of the level turn with entry velocity of $0.5 \mathrm{BL} / \mathrm{s}$. Given these results, upper bounds for the best observed turning radius for Finnegan in a $90^{\circ}$ are given by $1.54 \mathrm{BL}$ at $0.5 \mathrm{BL} / \mathrm{s}$ and $1.85 \mathrm{BL}$ at $0.75 \mathrm{BL} / \mathrm{s}$.

The trackline for case $\mathbf{D}$ in Figure 3-16 shows the result when the vehicle pitch actuation is saturated (with $\theta_{\beta}=45^{\circ}$ ) for all time after the roll angle reaches $90^{\circ}$. Case $\mathbf{D}$ demonstrates the turning radius for a $180^{\circ}$ or greater heading change, defined as the minimum distance needed in the direction perpendicular to the initial velocity, The position and orientation of the vehicle is shown at 0.5 second intervals, up to a heading change of $180^{\circ}$, in Figures 3-25 and 3-26. Position and orientation are plotted up until the vehicle reaches a heading change of $360^{\circ}$ in Figure 3-27, and velocity and 

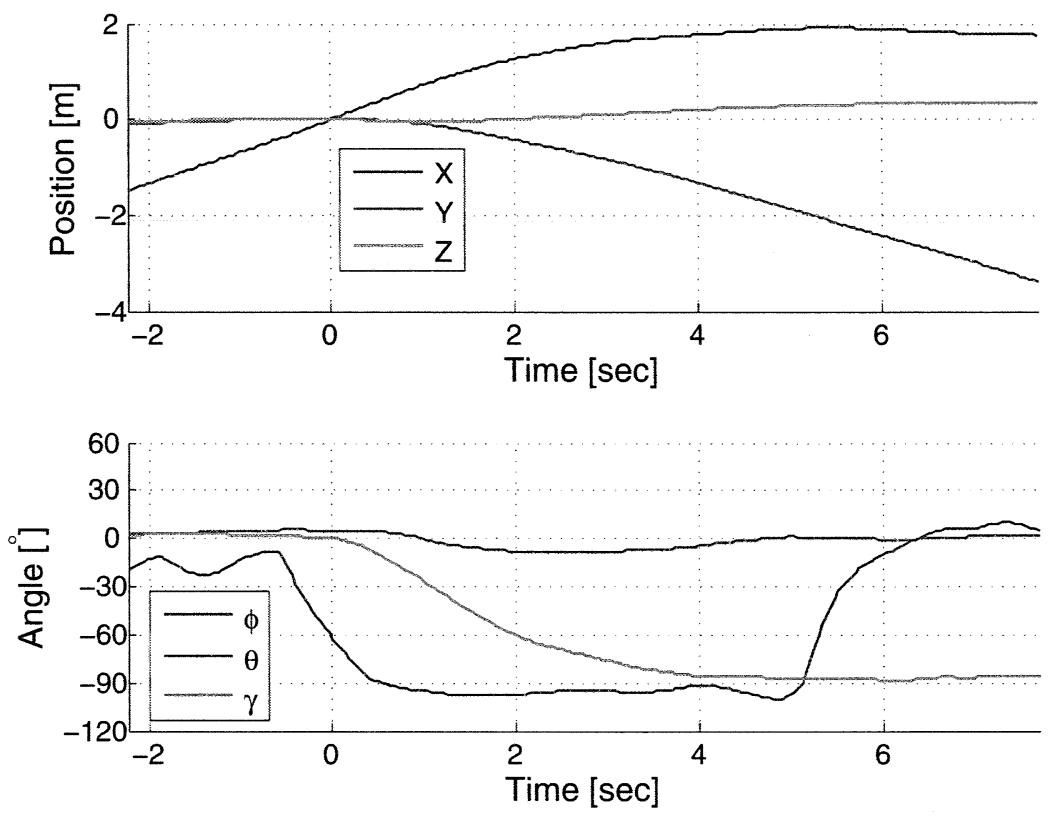

Figure 3-24: Vehicle position and attitude throughout banked turn. (Case B)

angular rates are plotted in Figure 3-28. $t=0$ where the roll angle initially exceeds $60^{\circ}$ and the heading reference is changed.

As shown in Figure 3-27, the vehicle reverses direction within 6 seconds, having translated on $2.0 \mathrm{~m}[1.54 \mathrm{BL}]$ in the $\mathrm{Y}$ direction, a turning radius of $0.77 \mathrm{BL}$. The foil positions and nominal angles of attack at the beginning of the turn are shown in Figure 3-29. The saturation in foil twist actuation during the turn is evident after $\mathrm{t}=2$ seconds in the $45^{\circ}$ postive twist bias on the forward foils (Foil 1 and Foil 3 as indicated in the figure inset) and the $45^{\circ}$ negative twist bias on the aft foils (Foil 2 and Foil 4.) The nominal angle of attack traces for the four foils clearly indicate the effect of a $45^{\circ}$ twist amplitude combined with $\pm 45^{\circ}$ twist bias - the forward foils achieve a maximum angle of attack of nearly $90^{\circ}$ on the downstroke and close to zero on the upstroke, while the aft foils achieve the high angle of attack on the upstroke and are highly feathered on the downstroke.

The ability to generate maneuvering forces with these high angles of attack, re- 


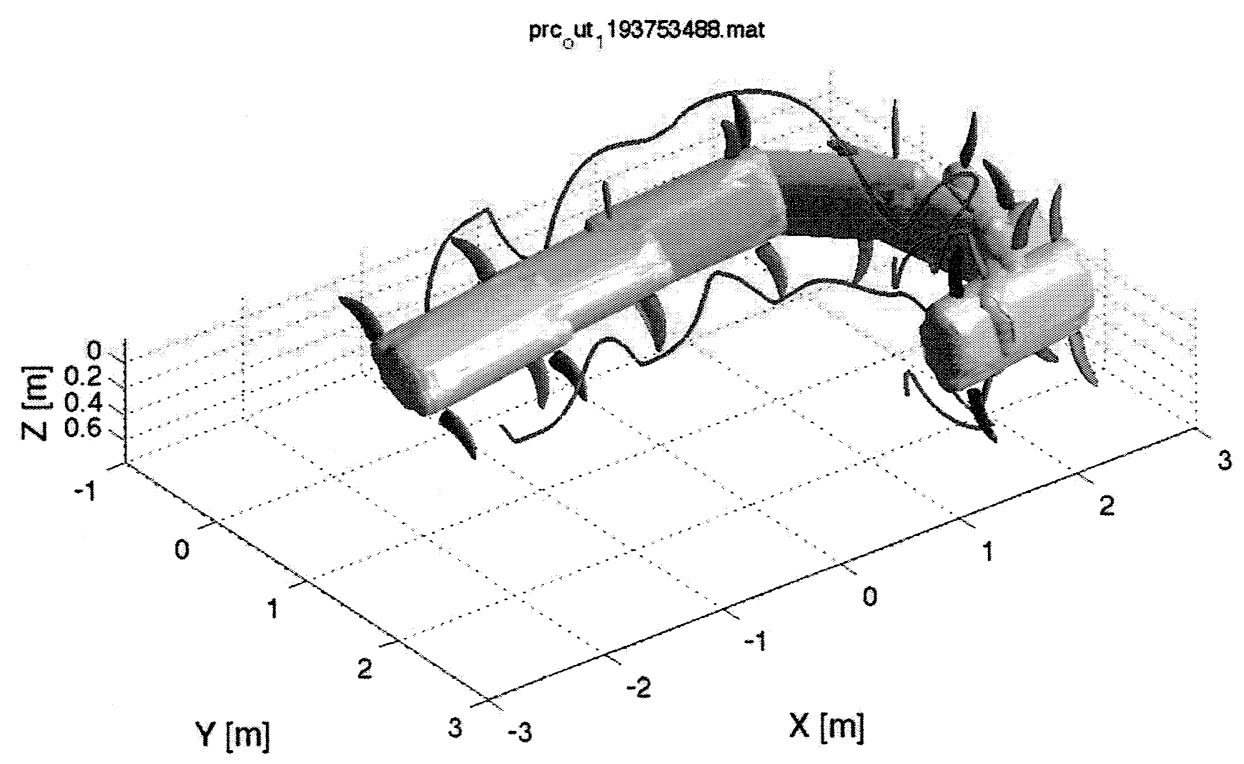

Figure 3-25: Visualization of a banked turn . (Case D) Vehicle and foil positions and orientations are shown at one second intervals, with lines tracing the path of the foil tips through space.

gardless of incoming flow velocity, is a key advantage for oscillating foils over conventional control planes. The vehicle forward speed drops from $0.98 \mathrm{~m} / \mathrm{s}[0.75 \mathrm{BL} / \mathrm{s}]$ to $0.25 \mathrm{~m} / \mathrm{s}[0.19 \mathrm{BL} / \mathrm{s}]$ in achieving a heading change of $180^{\circ}$ (see $u$ at $t=6 \mathrm{~s}$ in Figure 3-28) and approaches zero as the vehicle completes the $360^{\circ}$ turn. Throughout the entire turn, as the surge speed ranges from near maximum to zero, the vehicle is successfully stabilized about the desired roll and pitch angles, and the heading rate stays nearly constant, oscillating around $30^{\circ} / \mathrm{s}$.

For each speed tested, the variables during the turn expected to have significant, nonlinearly coupled effects on exit speed, turning radius, and maximum turning rate included:

- Roll, pitch and yaw gains during rolling phase

- Roll, pitch and yaw gains during pitching phase 


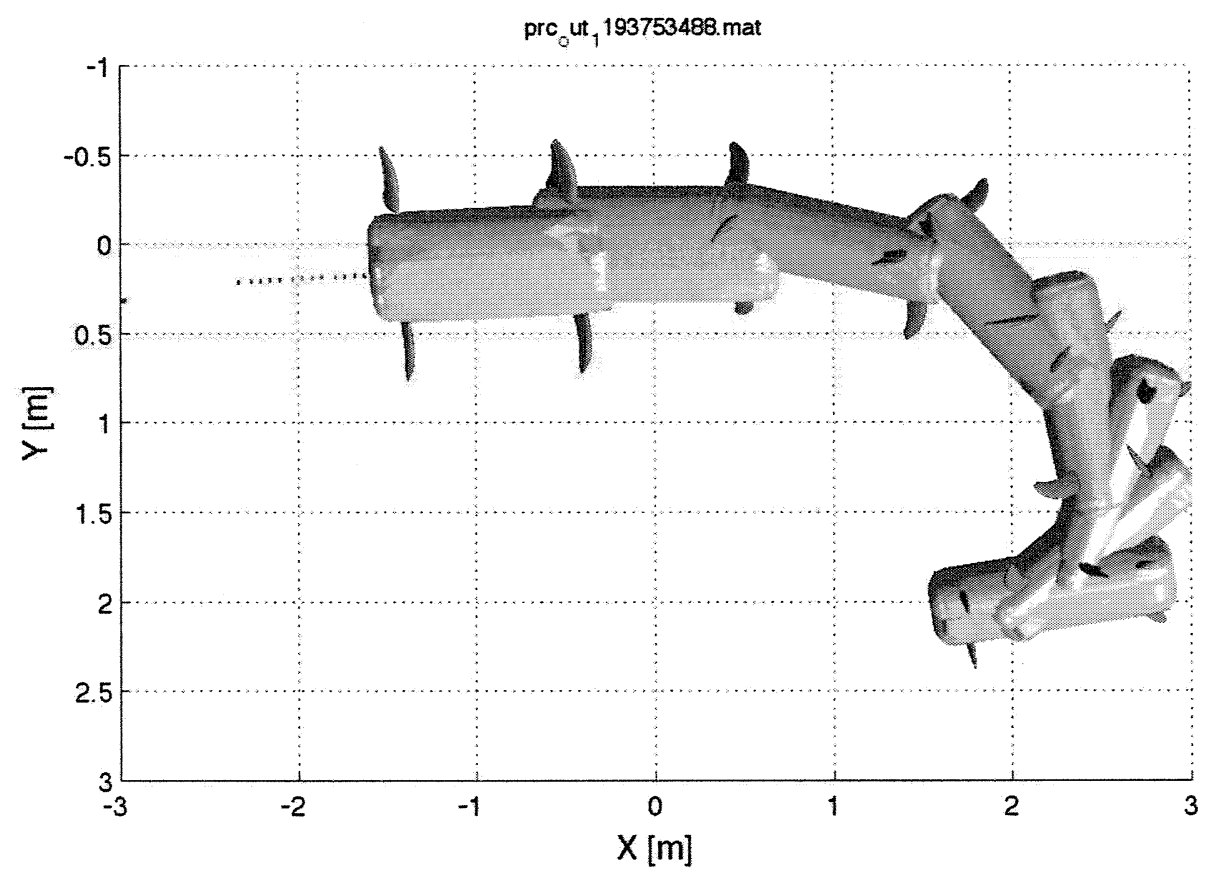

Figure 3-26: Top view of continuous banked turn with saturated body pitch actuation - (Case D) Vehicle and foil positions and orientations are shown at one second intervals.

- Roll, pitch and yaw saturation limits during rolling phase

- Roll, pitch and yaw saturation limits during pitching phase

- Oscillating frequency and baseline roll amplitude during rolling phase

- Oscillating frequency and baseline roll amplitude during pitching phase

Pool resources were limited to the extent that formal optimization across even two or three parameters was too costly, and with sixteen significant parameters there was no realistic option to pursue a comprehensive strategy. Furthermore, maximum performance as a function of a single parameter was often dictated by the actuation limits, but finding actuation limits for given set of constant parameters necessarily required operation to failure which was especially time intensive. The results highlighted here were chosen to illustrate the most successful turning performance. 

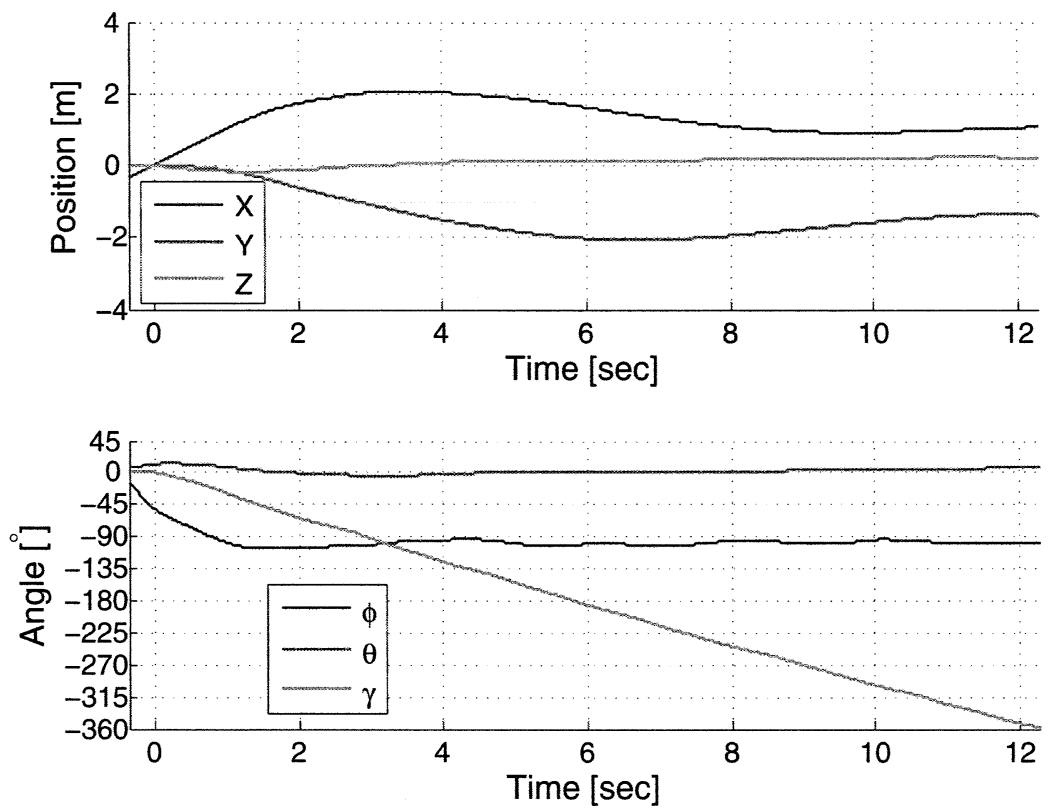

Figure 3-27: Vehicle position and attitude throughout continuous banked turn with saturated body pitch actuation. (Case $\mathbf{D}$ )

\subsection{3 "Twisting" Turns}

In addition to the 'one-at-a-time' style level and banked turns, where one angular reference value is changed at a time, a turning style where heading and attitude reference are changed simultaneously was tested with entry speeds of 0.5 and 0.75 $\mathrm{BL} / \mathrm{s}$. The body motion that results is not within the horizontal plane (heave control was not attempted during the course of the turn) and the vehicle gains altitude throughout the turn. The altitude gain occurs because of the manner in which the vehicle attempts to achieve the reference heading and attitude using the MRP based control algorithm. The controller approximately drives the vehicle to rotate about the constant axis which will take it from level to swimming on the side with heading perpendicular to the original track.

If the vehicle were hovering, pure torque would (ideally) be applied and the intermediate attitudes between the the starting and ending points could be ignored (as 

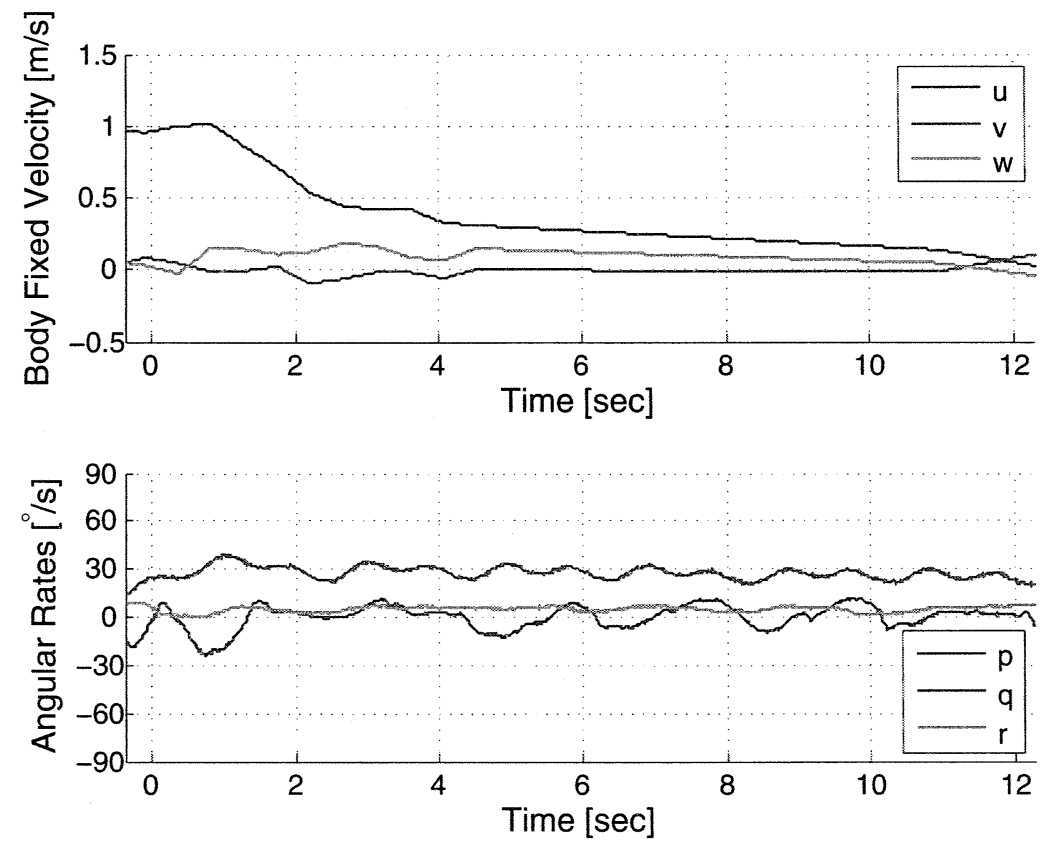

Figure 3-28: Vehicle position and attitude throughout continuous banked turn with saturated body pitch actuation. (Case D)

long as they do not pass through vehicle states that are not allowed.) However, in cruising mode the torque driving the vehicle body rotation is imposed on top of a steady forward driving force, and the intermediate attitudes have a direct effect on the direction of the self-propelled vehicle. With the axis of rotation as shown for a reference angle change of $\Delta \theta=\left[90^{\circ}, 0^{\circ}, 90^{\circ}\right]$ the attitude trajectory pitches the vehicle up as it rolls and turns to starboard, resulting in altitude gain.

Each test of the twisting style turn was halted after the vehicle achieved $90^{\circ}$ roll and $\Delta 90^{\circ}$ heading, but before the vehicle achieved zero pitch, due to pool depth limits. That the vehicle achieves $90^{\circ}$ roll and $\Delta 90^{\circ}$ heading before the pitch returns to zero is evidence that the axis of rotation was not maintained exactly throughout; while the controller guarantees stability for known torque input and low speed hydrodynamics, even under those conditions there is no expectation that the axis of rotation will be exactly maintained (recall that the vehicle dynamic and hydrodynamic parameters are not known, and that stability relies on the form of the equations of motion, rather 


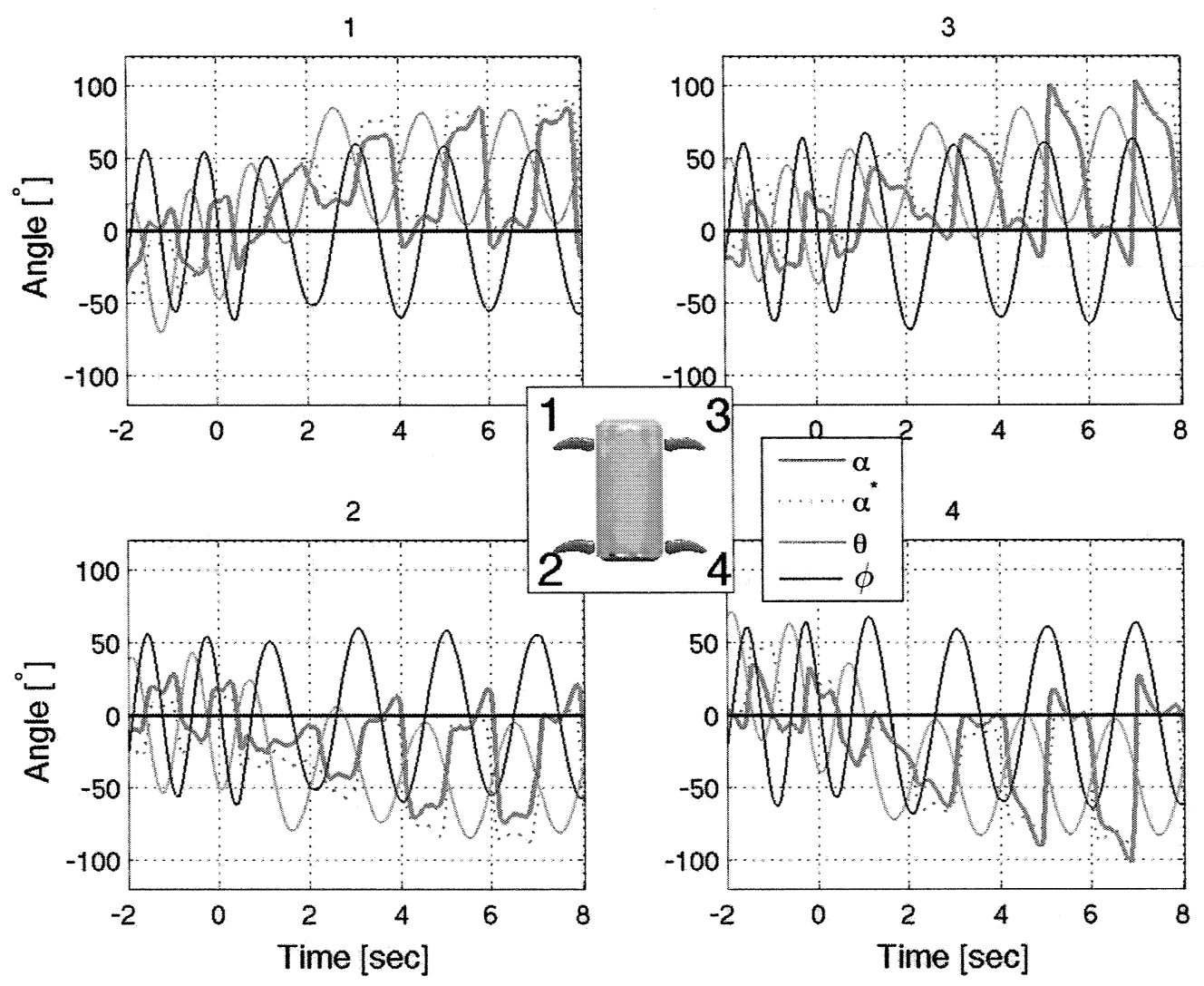

Figure 3-29: Roll angle $\phi(t)$ (blue), pitch angle $\theta(t)$ (green), and nominal angle of attack $\alpha(t)$ (red) resulting from foil motion, vehicle velocity, and vehicle rotation, at the beginning of a continuous banked turn with saturated body pitch actuation. (Case D).

than the exact values.)

\section{Kinematics for Selected Twisting Turns}

The tracklines for three different turns with two different entry speeds are included as Cases $\mathbf{F}, \mathbf{G}$, and $\mathbf{H}$ in Figure 3-16. Once again, the tracklines cross the origin at $t=0$ when the vehicle first begins attempting to actuate the heading change. All turns were performed to port to eliminate the effect of unknown asymmetries in vehicle mass distribution or geometry. For clarity $\mathbf{F}$, the turn with a nominal entry speed of $0.5 \mathrm{BL} / \mathrm{s}$ is shown turning to port, while $\mathbf{H}$ and $\mathbf{G}$, the turns with nominal entry speed of $0.75 \mathrm{BL} / \mathrm{s}$, are shown turning to starboard.

The baseline kinematics during all turns are identical in everything except sat- 


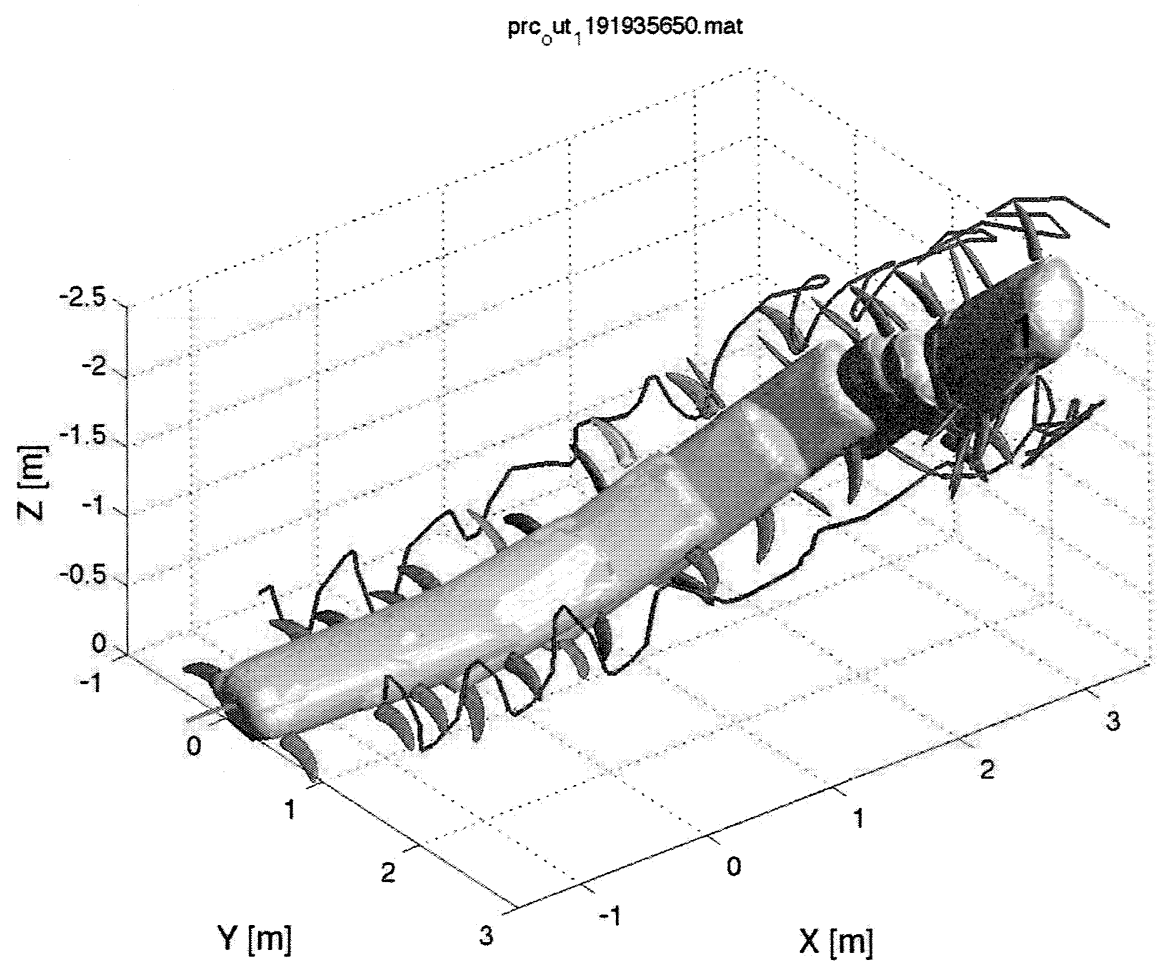

Figure 3-30: Visualization of a twisting turn . (Case F). Vehicle and foil positions and orientations are shown at one second intervals, with lines tracing the path of the foil tips through space.

uration limit: $f=0.5 \mathrm{~Hz}, \phi_{0}=60^{\circ}, \theta_{0}=40^{\circ}$. For case $\mathbf{F}$ and $\mathbf{H}$, the twist bias saturation limits are $45^{\circ}$, while for $\mathbf{G}$, the limit is $25^{\circ}$. The entry velocity for case $\mathbf{F}, 0.55 \mathrm{~m} / \mathrm{s}[\mathrm{BL} / \mathrm{s}]$, was achieved with cruising kinematics $f=1.0 \mathrm{~Hz}, \phi_{0}=30^{\circ}$, $\theta_{0}=30^{\circ}\left(S t=0.64\right.$ and $\left.\alpha_{\max }=34^{\circ}\right)$. The entry velocity of $0.75 \mathrm{~m} / \mathrm{s}[\mathrm{BL} / \mathrm{s}]$ for $\mathbf{H}$ and $\mathbf{G}$ is achieved with $f=1.0 \mathrm{~Hz}, \phi_{0}=60^{\circ}, \theta_{0}=45^{\circ}$ hence $\left(S t=1.1, \alpha_{\max }=37^{\circ}\right.$.)

\section{Comparison and Discussion of Results from Selected Twisting Turns}

The three banked turns shown achieved good performance in terms of forward obstacle avoidance distance of the dozen sets of kinematics tested, at $3.1 \mathrm{~m}$ [2.4 BL] for the entry velocity of $0.55 \mathrm{BL} / \mathrm{s}(\mathbf{F})$, and $3.5 \mathrm{~m}$ [2.7 BL] for an entry velocity of $0.75 \mathrm{BL} / \mathrm{s}$ $(\mathbf{H})$. To illustrate the typical behavior of the vehicle during a twisting turn, the vehicle position and orientation shown from two different views at 1 second intervals are depicted for case $\mathbf{F}$ in Figures 3-30 and 3-31. 


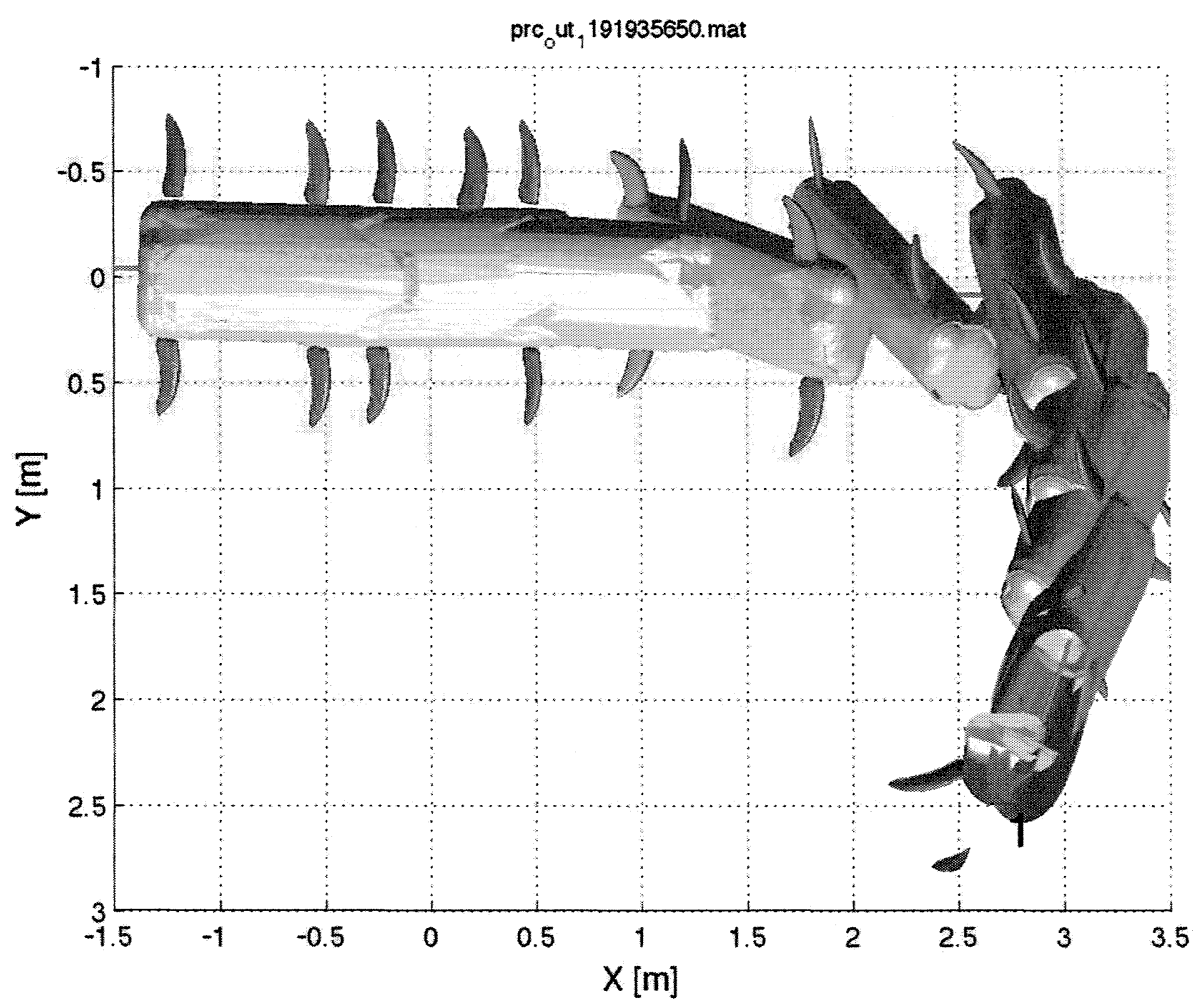

Figure 3-31: Top view of a banked turn . (Case F). Vehicle and foil positions and orientations are shown at one second intervals.

At first glance, using obstacle avoidance as a performance metric, the twisting turns are not as effective as the banked turns. However, if the time and distance required for the rolling maneuver at the beginning of the banked turn is included, the twisting turn outperforms the banked turn. Which comparison is more appropriate depends on external factors; both the geometry of the space in which the turn must be executed and the immediate mission requirements.

\subsection{Conclusion}

When aggressive maneuvering is required of an underwater vehicle, there are significant benefits to operating with roll/pitch instability, provided that the vehicle actuators are capable of rapidly vectoring force in response to the overturning moment, disturbances and pilot commands. The high authority, high bandwidth force production of oscillating foils is particularly well suited to this role, and a passively 

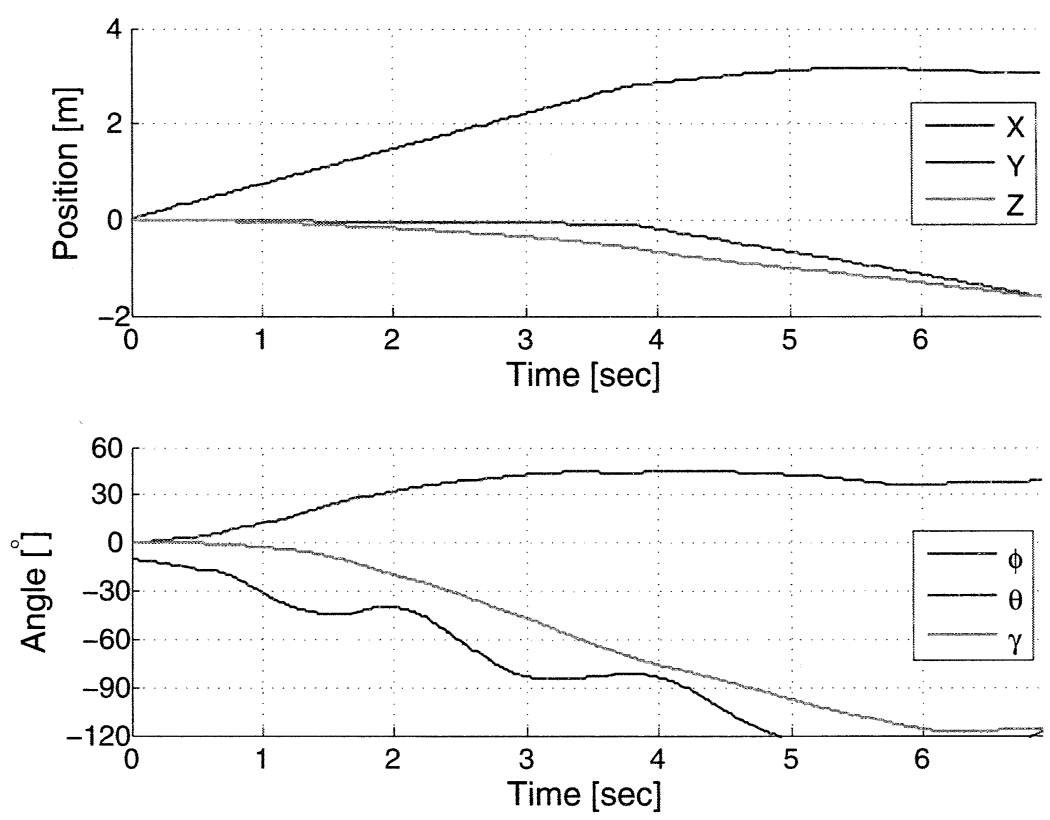

Figure 3-32: Vehicle position and attitude throughout twisting turn. (Case F)

unstable trim configuration was chosen to demonstrate the vehicle maneuverability gains that result from the enhanced force vectoring capabilities of oscillating foils.

Instability promotes maneuverability by making large angle maneuvers possible without requiring undue effort to travel away from a stable equilibrium point or points. Hence, in order to take advantage of the instability a control approach which can deal with large angles is required. The modified Rodrigues Parameters provide an excellent basis for formulating controllers for underwater vehicles rotating without restriction in roll, pitch, and yaw; straightforward controllers that are provably stable, despite parameter uncertainty, have been studied for underwater vehicles, although with no known practical application to date. Foils have been extensively studied experimentally, allowing the formulation of algorithms for force vectoring for an individual foil. Under assumptions about the applicability of data collected with unchanging foil kinematics to kinematics varying from stroke stroke, changes in lift and and thrust can be demanded from individual foils. These force vectoring strate- 


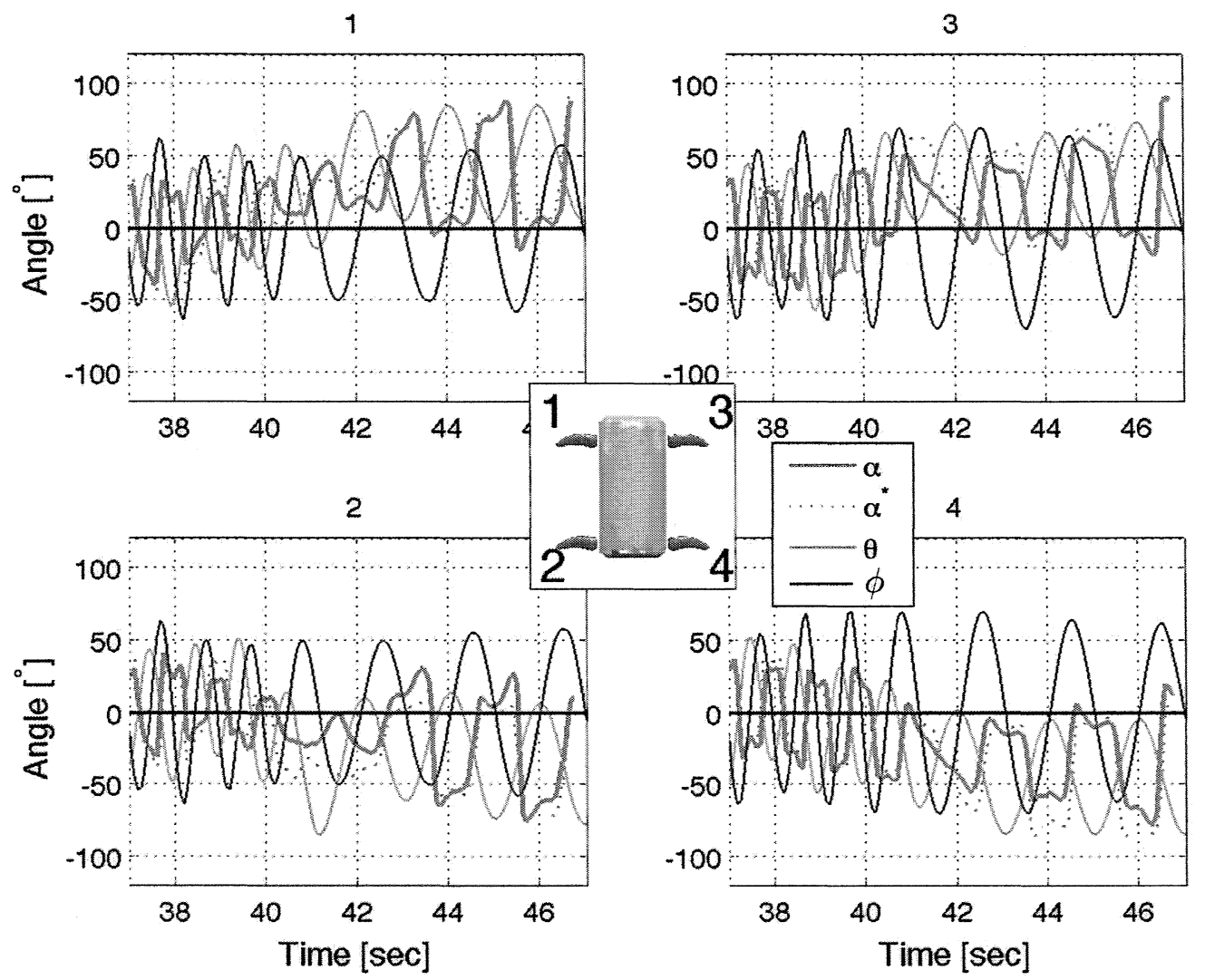

Figure 3-33: Roll angle $\phi(t)$ (blue), pitch angle $\theta(t)$ (green), and nominal angle of attack $\alpha(t)$ (red) resulting from foil motion, vehicle velocity, and vehicle rotation, throughout twisting turn. (Case F).

gies were combined with the torque demands of the controller based on the modified Rodrigues Parameters, and the resulting system was shown to be effective for basic maneuvering tasks in both hovering and cruising operation.

The maneuvering performance achieved during banked and twisting turns, which are particularly appropriate for an attitude unstable foil powered vehicle such as Finnegan, exceeds the best reported performance of existing AUV's by a factor of two. When the vehicle is rolled into the turn, the foils are oriented such that foil lift directly generates the steering moment. Significant lift forces can be generated with minimal reduction in thrust, and very large lift forces can be generated if thrust may be sacrificed for greater steering moment. During the level turn, steering moment can only be generated by changes in foil thrust, and the maximum available thrust 
is significantly lower than the maximum available lift. In Finnegan's case, the body shape is also more favorable to the turn when the vehicle is rolled to the side. The overall result is the the banked turn has a smaller turning radius, takes less time, and loses less forward speed than the level turn, supporting the observation that maneuverability capability can be improved through reduction in vehicle stability. 


\section{Chapter 4}

\section{Sea Turtle Maneuvering}

\subsection{Introduction}

While flexible bodies and conformable propulsive structures are nearly ubiquitous in marine animal locomotion, body flexibility dramatically reduces underwater vehicle payload space, and the advent of compact actuation for conformable fins awaits dramatic improvement in artificial muscle technology. The hard shelled Green turtle, Chelonia mydas, swims and maneuvers with a pair of high aspect ratio forelimbs in combination with a pair of low aspect ratio hind limbs. The hard shell and limited conformability of the limbs makes the Green turtle an excellent candidate to inspire vehicle design and control.

To determine how sea turtles use their limbs to control attitude and direction in confined spaces, I recorded and analyzed the limb kinematics and associated body motions of Myrtle, a green sea turtle Chelonia Mydas, residing in the Giant Ocean Tank of the New England Aquarium. Through the use of multiple cameras, I was able to capture her behavior while she was encouraged to maneuver in pursuit of food. A number of studies exist detailing the kinematics of steady swimming in juvenile and hatching sea turtles, and researchers have extensively studied the limb beat frequencies of sea turtle swimming during diving and foraging tasks, but no published studies of limb kinematics during transient maneuvers exist.

Myrtle was repeatedly filmed turning to locate and swim towards the diver, then 
pitching to bring her head close enough to the food to allow a downward (with respect to the body) neck extension/lunging motion to retrieve it. Periods of level swimming as well as the transition from level swimming or gliding to ascending for breath were also captured. These behaviors were observed both in the open water volume between the artificial reef and the tank walls, as well as in close proximity to, and in contact with, a diver or the reef.

Based on analysis of the video, the forelimbs were used in both drag and lift modes, as passive control surfaces, and as fenders against solid objects, sometimes all within the space of a few seconds during a single maneuver. Significant anterior and posterior components were observed during the all maneuvers, with the direction and magnitude of this inline motion depending on the type of maneuver being performed. The hind limbs, in addition to being used as control surfaces at times, were also recruited as propulsors to initiate rapid yawing turns, and to accomplish fine positioning tasks, during which they executed highly 3D motions.

\subsection{Experimental Procedure}

Digital video cameras (standard low- to mid-range consumer products of a variety of makes) were mounted on tripods on the public walkway which spirals up and around the Giant Ocean Tank. The cameras faced directly into the tank, perpendicular to the glass walls with the lenses as close as possible to the glass in order to eliminate glare from external light sources and to simplify the geometry for later self-calibration of the camera positions. Depending on the date of the experiment, two, three or four cameras were deployed.

In addition to the cameras outside the tank, either one or two video cameras with waterproof housings were placed inside the tank, whether mounted at an angle to the glass with suction cups to provide a view nearly perpendicular to the central external camera, or resting on the fiberglass faux reef structure in the center of the tank to

provide a view directly opposing the external cameras. The camera position on the fiberglass reef required the full time attention of a diver to keep animals, including 
Myrtle herself, from dislodging it, while the camera positions on the external walkway required the full time attention of an adult to ward off small children. The cameras were time-synced using a sharp metal on metal tapping noise at the beginning and end of the experiment.

The logistical restrictions on external camera placement (care was taken not to impede the walkway surrounding the tank,) and the limited number of usable internal camera placements, combined to produce a relatively small volume in which Myrtle could be observed from multiple viewpoints. To encourage her to swim and maneuver within this volume, a diver from the Aquarium research staff lured Myrtle by presenting food by hand, which she was obliged to retrieve by swimming in the camera views. Once she was within the camera volume, the divers would rapidly move the food above, below or behind Myrtle, causing her to maneuver reach them. Myrtle was typically willing to actively engage with the diver providing food for as long as an hour, provided that type of the food was varied whenever she began to lose interest. Several swimming events, particularly steady forward swimming against the slight prevailing current in the tank, and transition from level swimming to climbing to the surface for breath, were capture without the participation of the diver, as well.

Myrtle's mass, measured out of water on a scale within 3 months of the experiments, was $255 \mathrm{~kg}$. Her (curved) carapace length and width (measured at the same time as the weighing) are $1.12 \mathrm{~m}$ and $1.07 \mathrm{~m}$, respectively, with a circumference of $1.99 \mathrm{~m}$. The forelimbs have a span of $52 \mathrm{~cm}$ and maximum chord of $13 \mathrm{~cm}$, while the hind limbs are slightly wider, averaging $15 \mathrm{~cm}$ and approximately half the length, between $20 \mathrm{~cm}$ and $25 \mathrm{~cm}$. Flipper measurements were made in the water, and are approximate to within $3-4 \mathrm{~cm}$ 


\subsection{General Observations on Behavior and Limb Kinematics}

A number of useful general observations are possible before proceeding to analysis of selected maneuvering behaviors. Myrtle appeared to be negatively buoyant at all points within the $10 \mathrm{~m}$ deep tank. Myrtle controlled her position in the tank almost exclusively through actuation of yaw and pitch, whether during active maneuvering or steady swimming; Myrtle did not translate directly in sway or in heave, and rolled no more than 15 to 20 degrees from level during the experiments.

Body pitch ranged from -10 to +90 degrees during experiments. Myrtle preferentially swam with body pitch ranging from approximately 10-60 degrees when positioning herself for feeding or swimming steadily against the prevalent current within the cylindrical tank. She appeared to be stable in pitch and roll, but not so stable that her attitude was unaffected by the large roll and pitch moments generated during routine swimming or maneuvering.

When presented with food above her head, Myrtle pitched up as far as 90 degrees, using large amplitude forelimb motion, however, when presented with food below her head, Myrtle spiraled down with level body (i.e. body pitch $=0$ ) if unable to reach by extending her head downwards. When attempting to retrieve food or search for

food behind her, Myrtle changed her heading through yawing turns, rather than large angle maneuvering in roll or pitch.

\subsection{Detailed Analysis of Selected Behaviors}

The behaviors that were repeatedly observed during the experiments and chosen for further analysis were,

1. Rapid Pitching

2. Shallow Descent

3. Level Turning 


\section{Steady Swimming}

Strategies for rapid pitching and level turning are particularly germane to any discussion of large angle maneuvers for underwater vehicles, as many useful maneuvers can be be described at some level as a combination of yawing (turning), pitching and rolling within the body reference frame. The two maneuvers are adapted for execution on Finnegan the RoboTurtle in Chapter 5. Myrtle's behavior during shallow descent and steady swimming are particularly relevant to the discussion of the cost and benefits of asymmetric foil action, also discussed in more detail in Chapter 6 .

\subsubsection{Rapid Pitching}

Myrtle was observed rapidly pitching upwards and ascending, both prompted and unprompted by the diver offering food. When prompted by food presented above her head, Myrtle typically pitched upwards rapidly and then allowed both her forward and her upward motion to stall while craning her neck to reach the food. During unprompted ascents to breathe at the surface Myrtle typically continued to use both forelimbs to swim up and out of the camera viewing area.

Pitch was initiated with large amplitude synchronous forelimb downstroke, with a moderate anterior component, and a high induced angle of attack during the fastest portion of the stroke. The initial downstroke was followed by a highly feathered upstroke with approximately the same duration as the downstroke. The hind limbs were stretched out to the side and held nearly horizontal (i.e. parallel to the ground) throughout the motion, presumably either acting as passive control surfaces or simply reducing the opposing pitch moment created by drag (both reducing drag directly by presenting a lower angle of attack, and by bringing them closer to the center of gravity)

Through this combination of limb action, Myrtle was able to achieve pitch angles of up to 80 degrees within a single cycle of forelimb motion. In Figure 4-1, Myrtle is motivated to pitch by the diver offering food above here head, hence she uses just a single stroke in order to put her head in range of lunge for the foodl. From $t=0$ to 


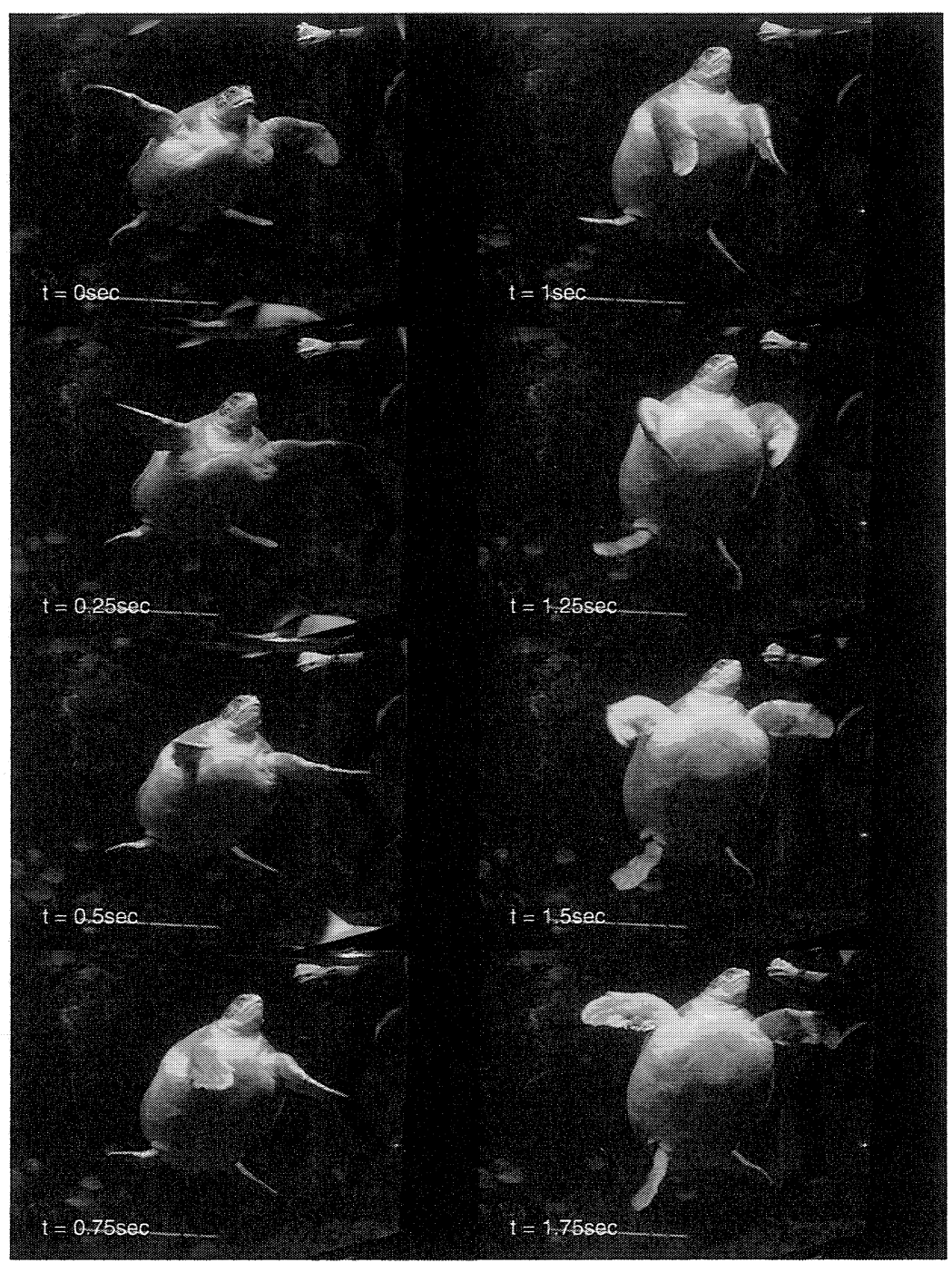

Figure 4-1: Front view of rapid pitching maneuver to retrieve food. Myrtle achieved a high pitch angle through a single rapid down- and forward- stroke of her pectoral fins with a high angle of attack to the flow. 


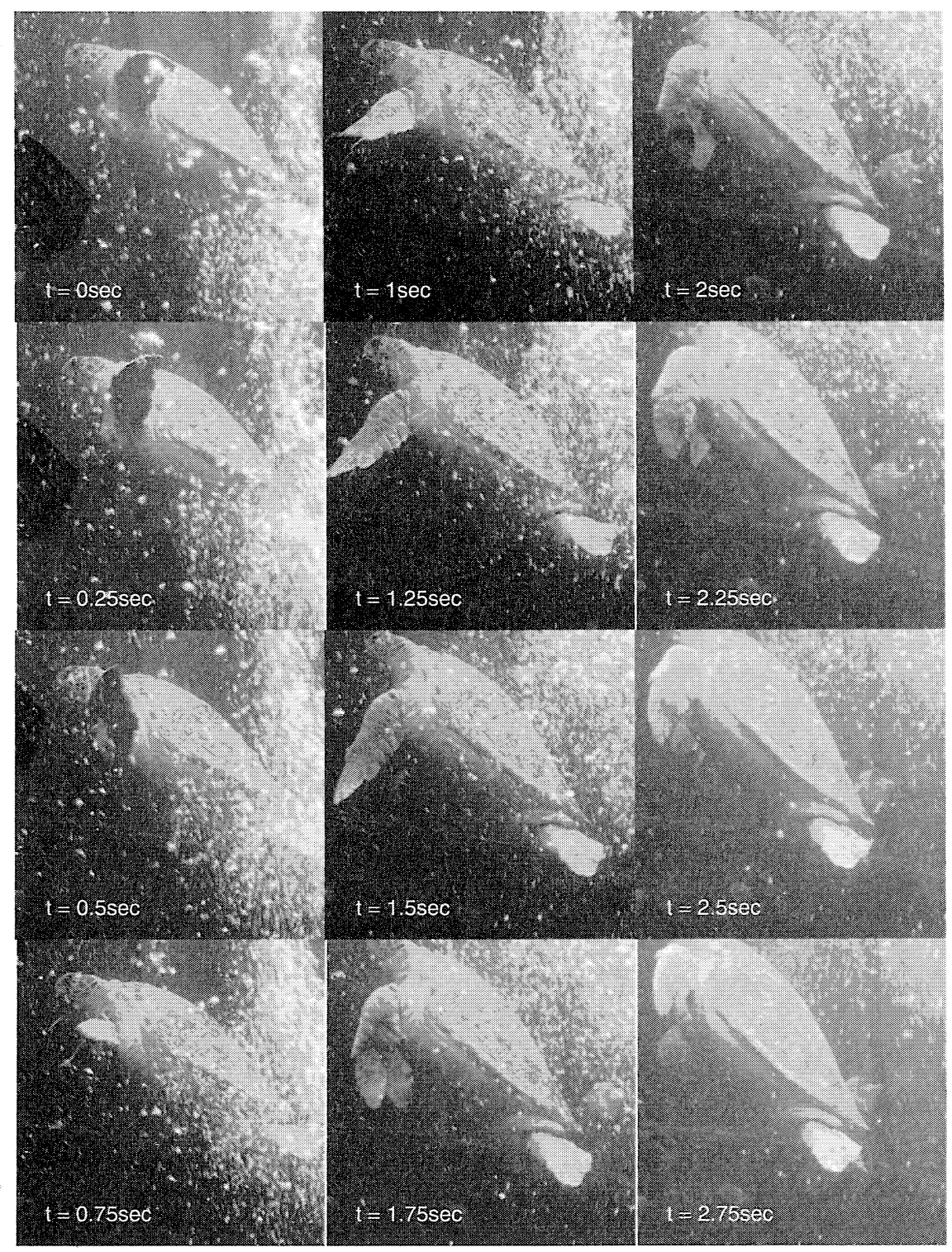

Figure 4-2: Side view of rapid pitching maneuver in preparation for swim to surface. Myrtle maintained forward speed while pitching upwards, utilizing less foil motion inline with the flow than in Figure 4-1, where the forward motion was halted during a more rapid pitching maneuver. 
$\mathrm{t}=0.75$ seconds, the synchronous downstroke which initiates the pitching maneuver is accompanied with a significant anterior component, the forelimbs sweeping forward to arrest forward motion and generate a pitching moment. Between $t=0.75$ and $t=1.0$ seconds, the flippers recover from the downward and forward position through primarily posterior motion. From $\mathrm{t}=1.25$ to the last frame at $\mathrm{t}=1.75$ seconds, Myrtle performs a highly feathered upstroke as her ascent stalls at the desired depth, and finally she cranes her neck to reached the proffered food. From this angle, it is clear that the hind limbs are held at a low angle of attack to the flow until $t=1$ second. From $\mathrm{t}=1.25 \mathrm{t}=1.75$ seconds, the hind limbs may be used to maintain depth and tilt the body towards the diver with drag based thrust generation.

In contrast to the previous example, in Figure 4-2, Myrtle is pitching up with the intention of swimming to the surface, and continues to execute swimming strokes after she exits the last frame. As a result, she is motivated to maintain surge speed even as she pitches up to a significant angle. In this case, the very beginning of the synchronous downstroke which initiates the pitching maneuver, from $\mathrm{t}=0$ to $\mathrm{t}=0.25 \mathrm{~s}$, is accompanied with some forward motion. However, rather than arresting forward motion of the body, this foil motion appears to be intended to set up the rest of the downstroke, during which the forelimbs are swept backwards with respect to the pitching and surging body, most importantly during the highest velocity portion of the downstroke from $t=0.5$ to $t=1.0 \mathrm{~s}$. The portion of the upstroke which is visible before Myrtle swim out of frame (from $t=1.75$ on) is a highly feathered recovery stroke which brings the forelimbs back forward level in preparation for the next downstroke. The period of the total stroke here is greater than 2.75 seconds, in comparison to 1.75 seconds in the prior example.

\subsubsection{Shallow descent}

Myrtle nearly uniformly responded to the presentation of food above her head by pitching her body upwards radically. However, when presented with food below and in front of her, Myrtle appeared to prefer to allow herself to passively sink slowly as a result of her overall negative buoyancy. In general, Myrtle appeared to be reluctant to 


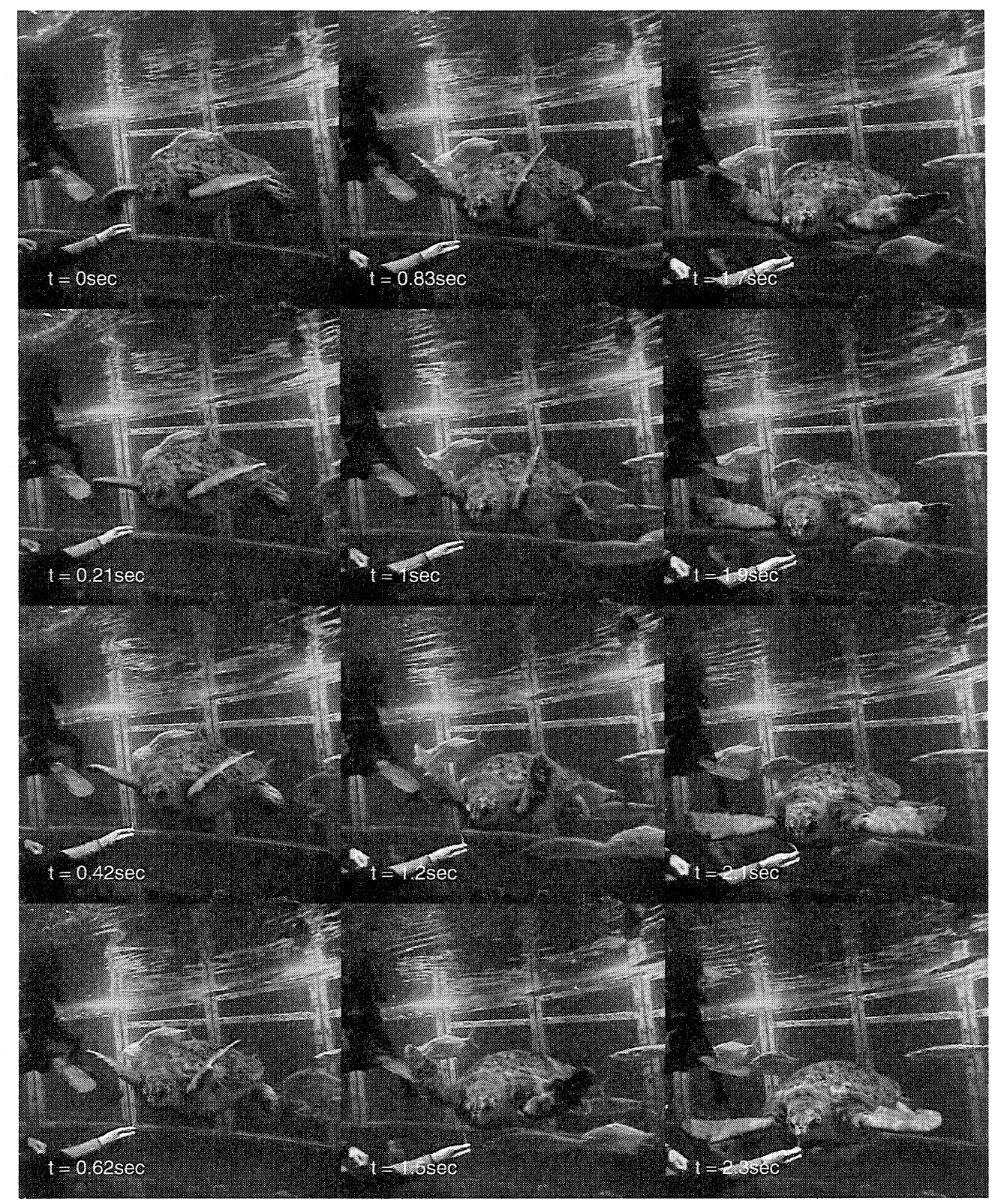

Figure 4-3: Front view, swimming to target ahead and slightly below. Myrtle utilizes a fore-aft rowing stroke with her forelimbs rather than using a lift based stroke which would tend to pitch her up and away from the proffered food. 
swim with extreme negative body pitch angles, possibly because of her limited ability to raise her head (limited in comparison to the large range of motion for her neck and head below her shell.) Faced with the need to propel herself forward in surge in order to reach the food, Myrtle would utilize a fore-aft rowing stroke with her forelimbs as she sank, rather than using a lift based stroke which would counterproductively tend to pitch her up and counteract the sinking motion. This is a strong indication that Myrtle has limited capacity to generate thrust on the upstroke, as expected from her observed preference for a fast downstroke and slower, feathered upstroke in almost all swimming tasks.

In general, when accelerating from a gliding state (i.e. with forelimbs extended to the sides, and flippers nearly horizontal) towards food presented ahead and below, Myrtle would start with a low speed upstroke, dominated by anterior motion with minimal transverse motion, highly feather. Forward thrust was then generated by a rowing power stroke dominated by posterior motion with the flippers held nearly vertical, i.e. perpendicular to the limb motion through the water. Body pitch angle throughout was maintained near level, or slightly negative.

In Figure 4-3, Myrtle is seen from a front view swimming to a target ahead and slightly below. From $\mathrm{t}=0$ to $\mathrm{t}=0.83$ seconds the forelimbs perform a low amplitude, low speed upstroke with some anterior component. This is followed by a power stroke dominated by posterior motion, with the flippers held nearly vertical during the fastest section of the stroke (around $t=1.7 \mathrm{sec}$ ) resulting in a high angle of attack rowing mode of thrust generation. The power stroke ends with the fore flippers once again extended to the sides with the flippers horizontal, minimizing drag $(t=2.3 \mathrm{sec}$.) Body angle with respect to horizontal is maintained near zero throughout. Figure 4-4 shows a side-rear view of the same maneuver, with the frames corresponding in time between the two views.

\subsubsection{Level Turning at Low Speed}

Myrtle accomplished level turns out of a glide motion by recruiting one, two or four limbs for thrust generation. The forelimb on the inside of the turn was used both as 


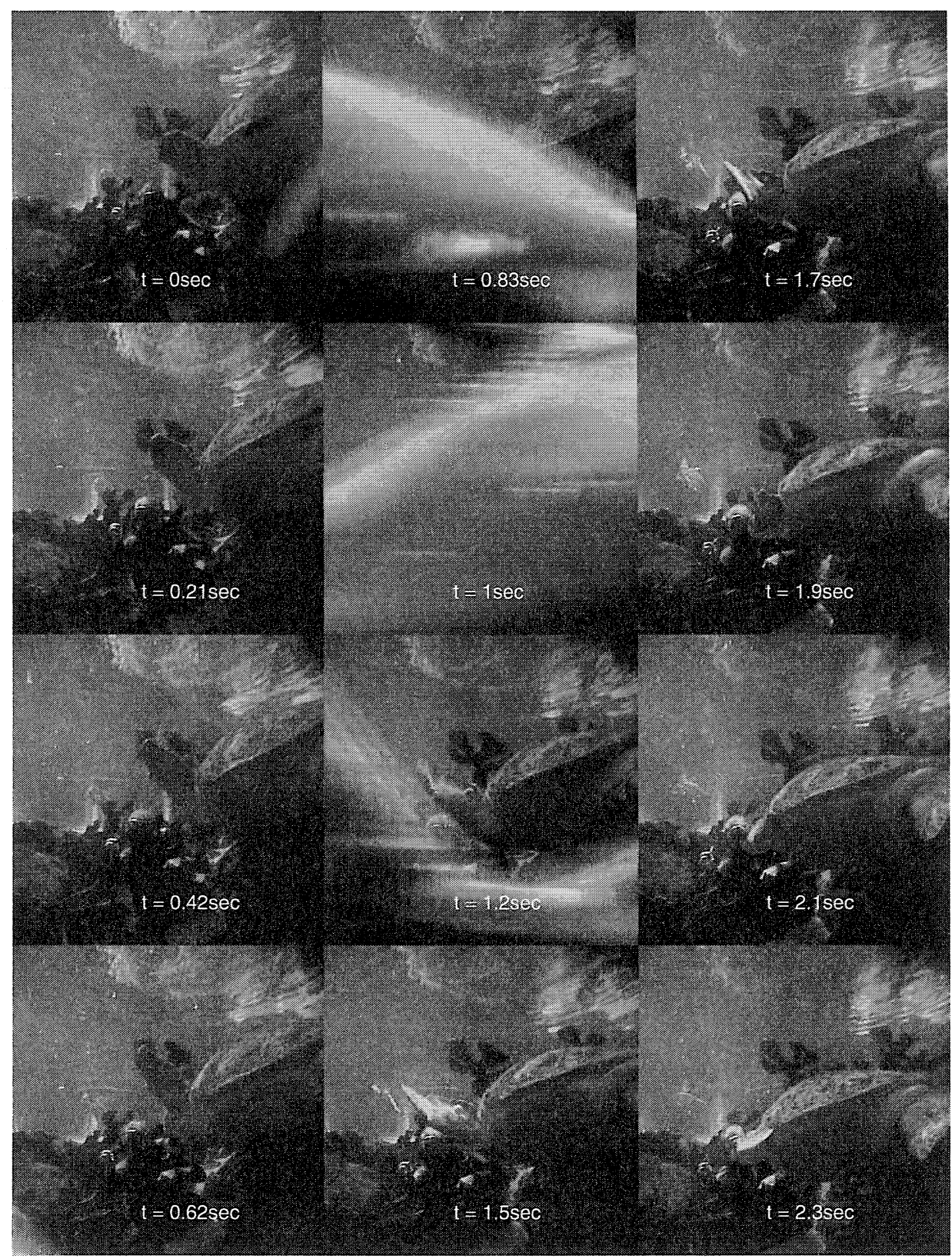

Figure 4-4: Side/rear view of swimming to target ahead and slightly below (simultaneous with view shown in Figure 4-4. The nearly vertical state of the pectoral fin surface is particularly evident from this view. (The frames at $t=0.83$ and $t=1$ second were obscured by a fish swimming in front of the camera.) 
a control surface, whether a rudder or a brake, as well as in propulsion/roll control through a drag dominated rowing stroke, or it was simply swept back to the shell and held out of the flow. The forelimb on the outside of the turn was uniformly used in a lift based stroke, resulting in an unbalanced forward thrust which created a turning moment. The outside limb was swept forward with respect to the body and with respect to vertical at to the top of the stroke, and brought down vertically on the downstroke, resulting in posterior motion with respect to the body. When both forelimbs were active, the motion of the inside forelimb led that of the outside forelimb by as much as $0.17 T$, where $T$ is the total period of the a completed stroke. (Throughout this section, on a turn to starboard, the starboard limb is the inside limb and the port limb the outside limb. On a turn to port, the port limb is the inside limb and the starboard limb is the outside limb. Myrtle was observed turning in both directions without apparent preference.)

Turning was generally initiated from a glide position by the hind limbs, either with a vigorous simultaneous rowing sweep with the ends of the flippers moving in the opposite direction from the turning direction or with a less dramatic use of the inside hind limb in a rudder like fashion. Both approaches end with the hind limbs in the same position.

As the body begins to turn the outside forelimb is brought up and then forward with respect to the body, with the blade feathered. While the outside forelimb is moving forward, the inside forelimb is either

1. swept downwards from the horizontal to vertical or past vertical in a drag based rowing motion which generates both a sway force and yaw moment

2. brought downwards more slowly and held with the blade perpendicular to the surge direction, acting as a brake which retards forward motion while generating a turning moment

3. held close in towards the body

4. or held close to horizontal with the blade near level. 


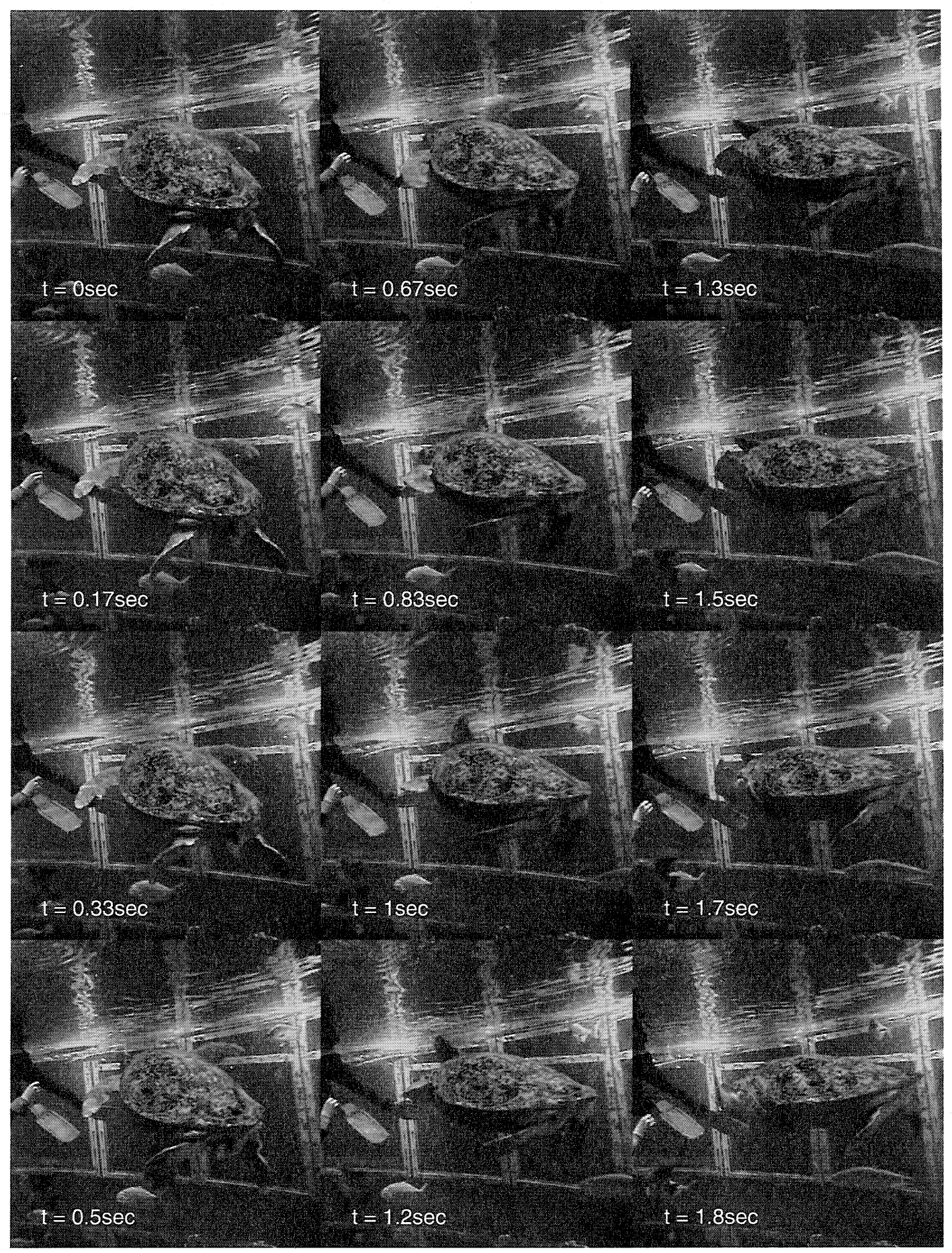

Figure 4-5: Part of a representative level turn using rear limb rapid paddling stroke and inline motion of outside forelimb. The inside forelimb is held level at first, then joins the outside forelimb in a fore-act rowing motion to slow the turn. 


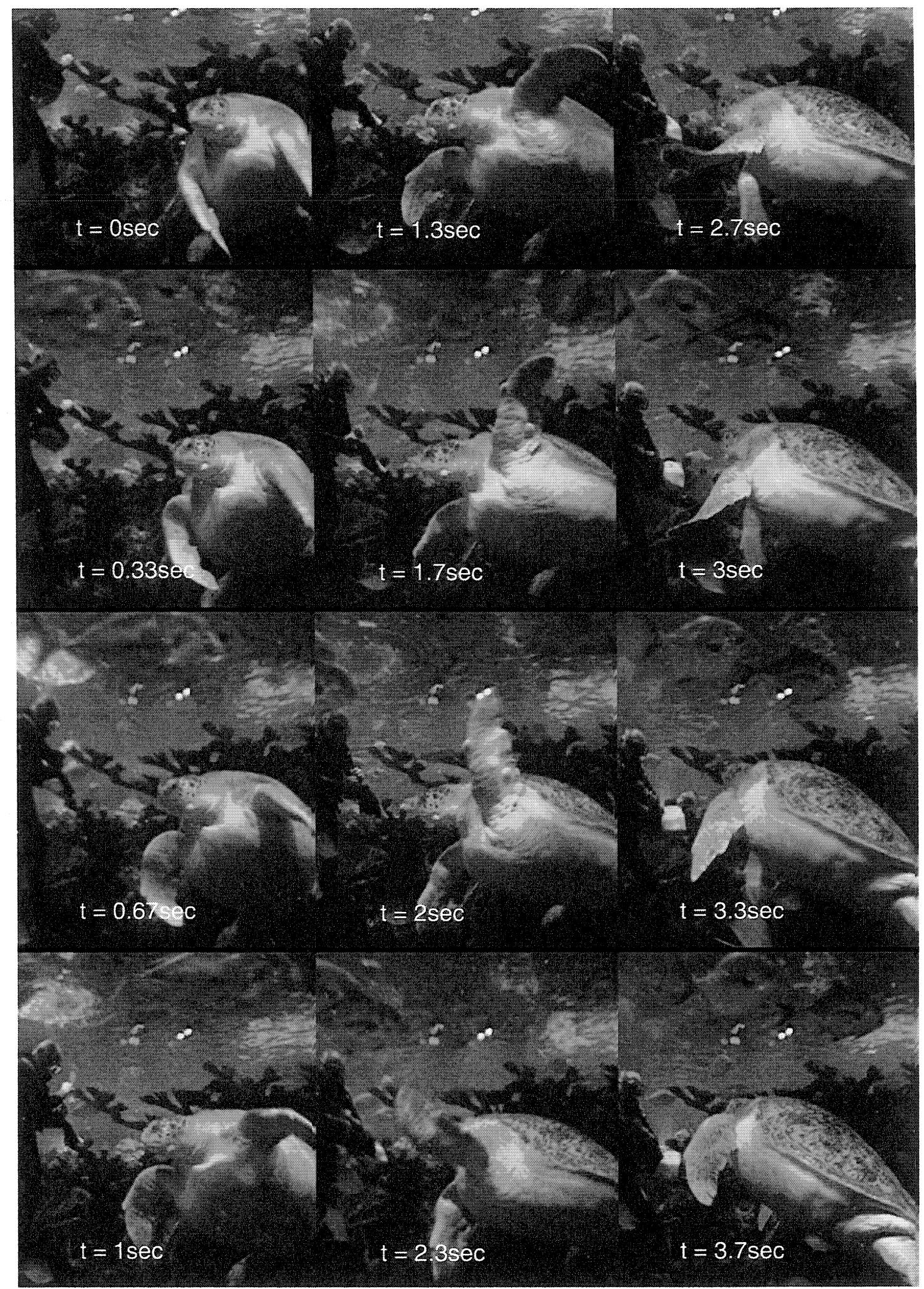

Figure 4-6: Front view of part of representative level turn with active participation of both forelimbs. Frames start midway through the turn: $t=0$ sec corresponds to the end of the first downstroke of the outside forelimb during this turn. 


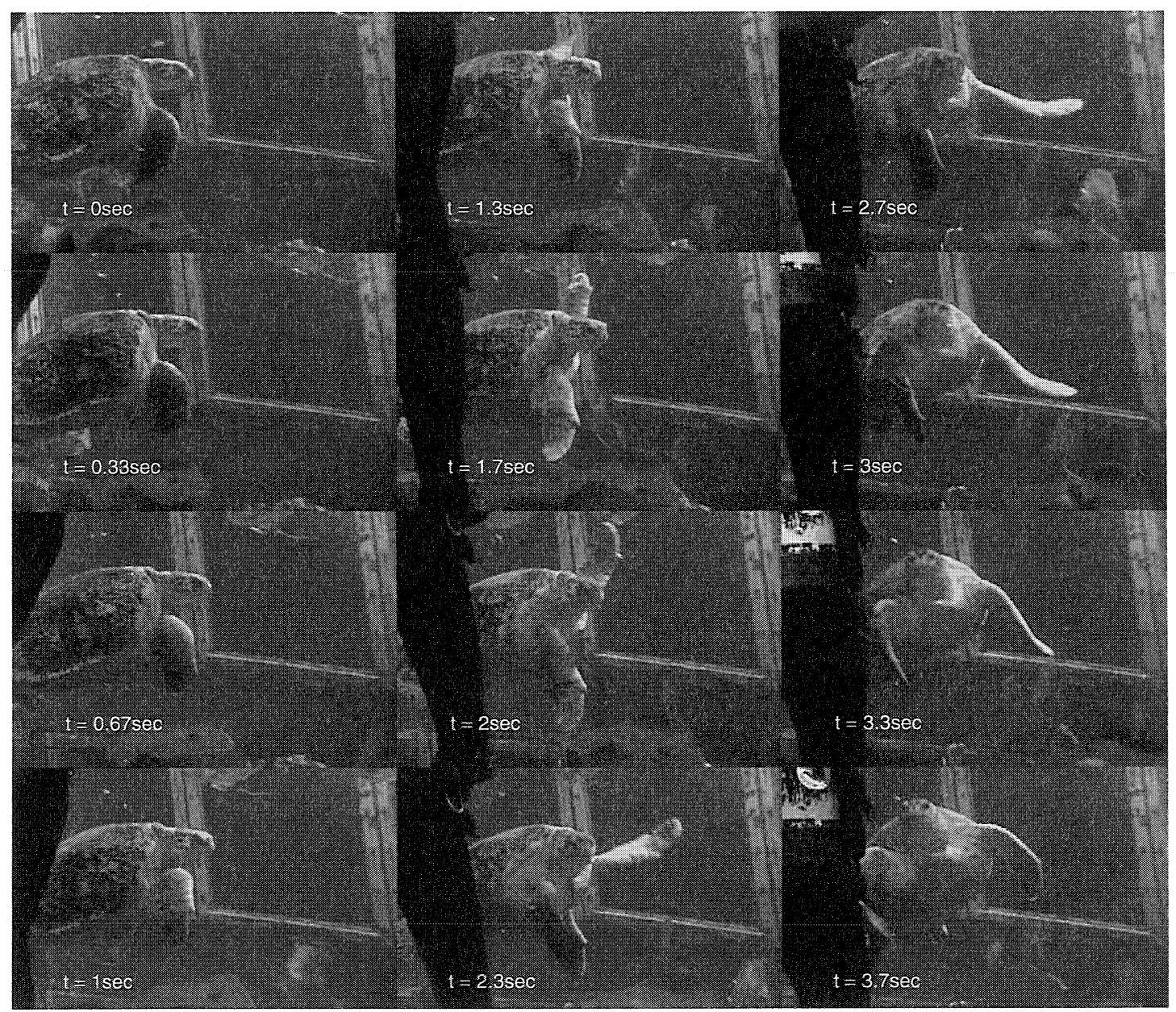

Figure 4-7: Side view of representative level turn with active participation of both forelimbs (simultaneous with Figure 4-6. This view highlight the extension of the outside forelimb as it is thrown forward just as the downstroke is initiated.

The outside forelimb begins a lift based downstroke which generates thrust from the right side of the body, resulting in a turning moment. If the inside forelimb is actively stroking, the downstroke of the outside forelimb lags the downstroke of the inside forelimb. The accompanying posterior motion of the flipper with respect to the turning body results in a near vertical motion of the flipper tip with respect to the fluid. If the turn is to continue, the outside forelimb is raised back to the starting position of the first downstroke with a highly feathered recovery upstroke, while the inside forelimb, if stroking, is brought back to some angle well short of horizontal before a second downstroke commences with the same lag time between the two limbs. 
Screen captures at regular time intervals are shown for two turns. The first, in Figure 4-5 shows the start of a turn where the inside forelimb is held horizontally out from the body, illustrating the use of a rapid leg kick. The second turn, from different viewpoints in Figure 4-6 and Figure 4-7 details a turn with active participation of both forelimbs, shown from the end of the first downstroke to the end of the second downstroke.

In Figure 4-5, turning is initiated from a glide position by the hind limbs, with a vigorous simultaneous rowing sweep between from $t=0.33$ and $t=0.67$ seconds. The hind limb motion alone results in a nearly 60 degree heading change between $t=0.33$ and $t=1.33$ seconds, during which time the outside forelimb has been raised up and pushed forward to prepare for the lift based downstroke to follow. The inside forelimb is held straight out from the body, with surface horizontal.

In Figure 4-6, both forelimbs are near the maximum possible downward excursion in the first frame at $t=0$ seconds. From $t=0$ to between $t=1$ seconds, both limbs are in the recovery stroke. While the recovery stroke of the outside limb continues until between $t=1.7$ and $t=2$ seconds, the upward stroke of the inside limb ends around $\mathrm{t}=1.3 \mathrm{sec}$, after which it starts a downward sweep with the blade perpendicular to the resulting flow. The downward stroke of the inside limb continues through to $\mathrm{t}=2.7$ seconds, during which time the outside limb has completed significant anterior motion with the blade feathered and begun the lift based power downstroke, which continuing to the last frame at $t=3.7$ seconds. From this view it is apparent that while the motion of outside limb contains a significant posterior component with respect to the body, is brought down nearly vertical with respect to the world frame as a result of the turning motion of the body. Figure 4-7 shows the same turn from a different angle, where it is clear the that inside forelimb downstroke ends with the limb well past vertical underneath the body. This view also illustrates the effect of the forward motion of the outside forelimb - contrast the forelimb position in frames at $t=1.3$ and 1.7 seconds to the forelimb extension from the frames from $t=2.3$ to $t=3.7$ seconds. Myrtle achieves a heading change of between $80^{\circ}$ and $90^{\circ}$ during the period pictured here, for an average heading rate of between $21^{\circ} / s$ and $24^{\circ} / s$. 


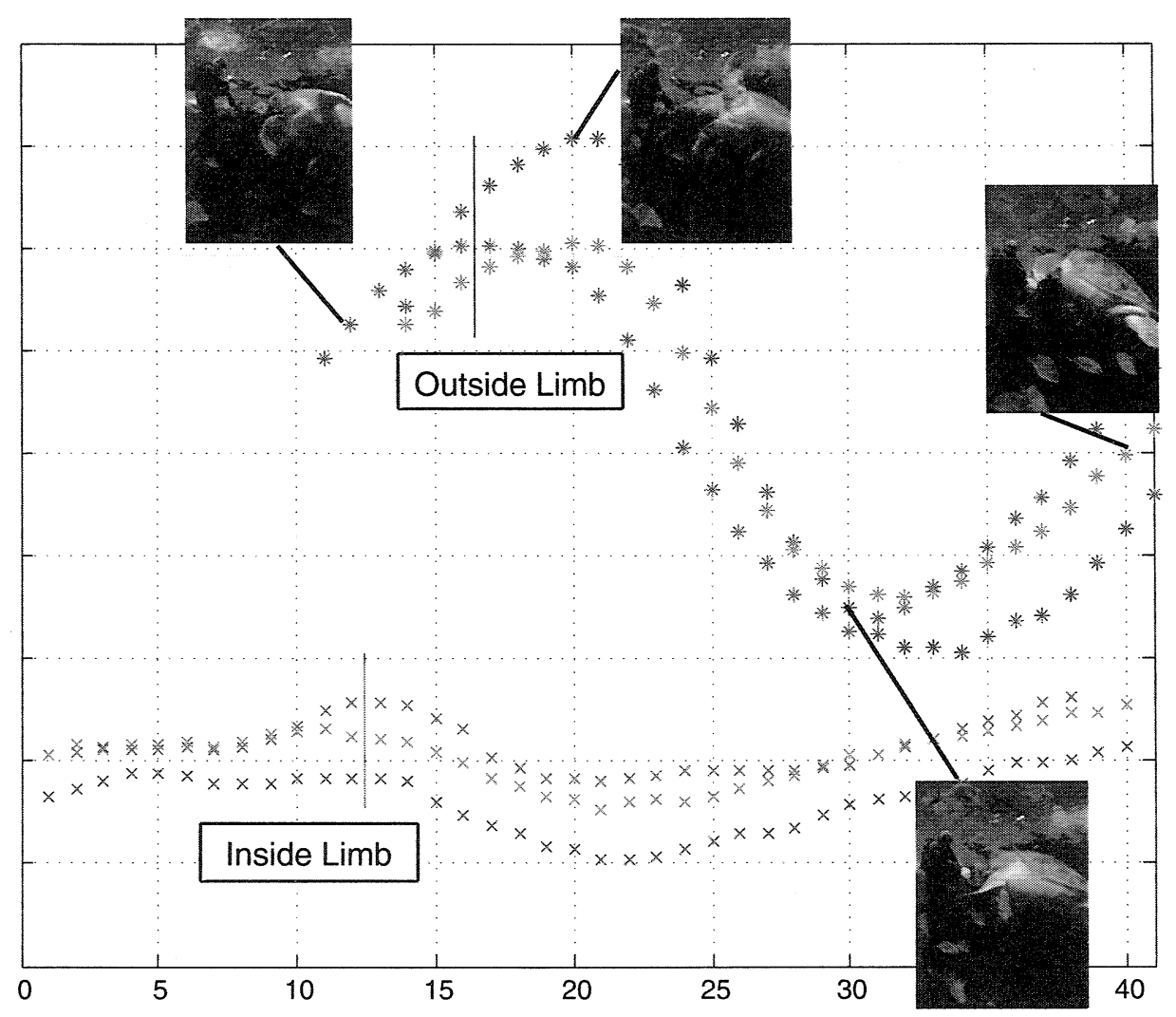

Figure 4-8: Limb tracking data for turn with active participation of both forelimbs shown in Figure 4-6 and Figure 4-7. Blue, green and red correspond to the foil tip, leading edge and trailing edge at join between upper and lower limb sections, respectively. Y-scale is normalized pixel location on the side camera view, shown in inset pictures.

To clarify the lag between the inside an outside forelimb strokes during turns where both limbs are actively stroking, the position of the limb tips and of the break between upper and lower limbs segments at the leading and trailing edges were tracked at a frame rate of $10 \mathrm{~Hz}$. Red, white and yellow dots in selected frames in Figures 4-7 and the squares in selected frames in Figure 4-6 indicate the points that were tracked whenever visible in either camera. The y-locations of each point through time are plotted (with identical color/shape scheme) in Figure 4-8, normalized by maximum and minimum pixel location for appropriate camera. Using the leading edge joint position, the foil upstroke and downstroke period for the outside limb are nearly identical, at $T_{d}=1.4 \pm 0.1 \mathrm{~s}$ and $T_{u}=1.6 \pm 0.1 \mathrm{~s}$ respectively. The time lag between 
the start of the downstroke for the two limbs is approximately $0.5 \mathrm{~s}$, or $\left(T_{d}+T_{u}\right) / 6$.

\subsubsection{Straight Line Swimming}

Myrtle was observed during multiple instances of steady swimming as she circled around the tank against the slight prevailing current. During all the instances recorded in detail below, she swam within a body length of the tank window, which forced her to adjust her heading slightly throughout. Her depth and body pitch angle vary within a few meters and approximately 30 degrees within each recorded instance of steady swimming. Myrtle was also observed accelerating straight and level from a nearly stationary state.

- The forelimbs remained in phase during steady swimming.

- A limited anterior/posterior component to the forelimb motion was observed during steady swimming.

- The constant change in heading required to follow along the tank wall was accomplished by periodically increasing the maximum excursion of the outside forelimb, which ranged from 30 to 70 degrees during observations of steady swimming

- The downward excursion of the forelimbs was nearly constant, at approximately 90 degrees from horizontal.

- Depth and pitch were controlled using the hind limbs as elevators.

- The hind limbs were held back in a streamlined position when not controlling pitch.

- Speed was controlled using with forelimb frequency - to accelerate from a standing start, forelimb frequency was double that of the steady swimming case.

- The duration of the forelimb down stroke during throughout observed level forward swimming ranged from 1.0 seconds (recorded during acceleration) to 1.7 seconds. 


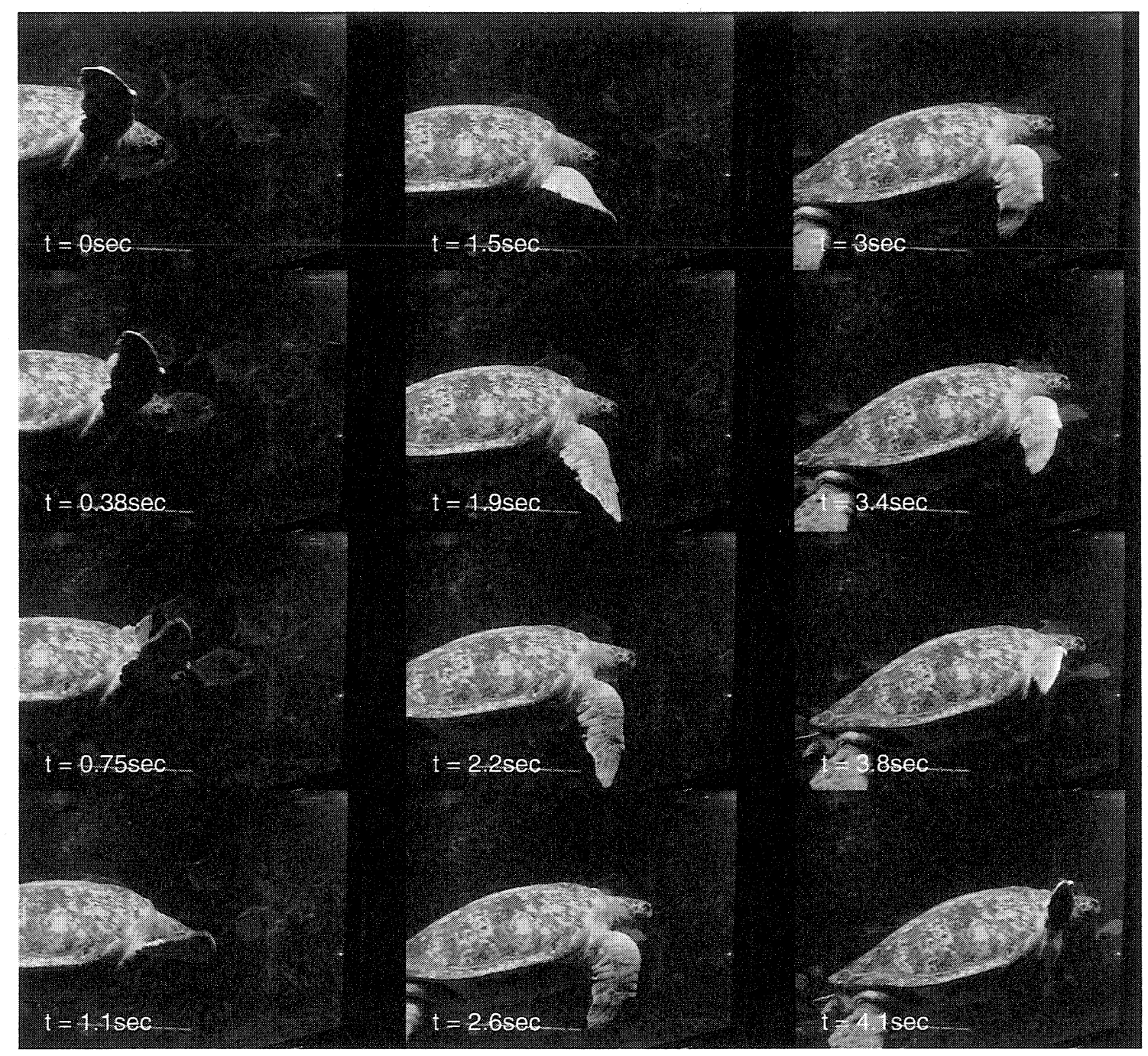

Figure 4-9: Side view of representative steady swimming stroke.

- The duration of forelimb up stroke ranged from 1.5 seconds (recorded during acceleration) to 2.7 seconds.

- The ratio of down stroke to following upstroke period ranged from 0.6-0.7.

Figure 4-9 shows a side view of Myrtle swimming past a single camera, which is set back from and at angle to the aquarium window. This view illustrates typical variation in body pitch angle on the time scale of a single stroke, as well as the lower twist angle of the downstroke as compared to that of upstroke, which results in a higher angle of attack, greater thrust force, and higher torque requirements. 


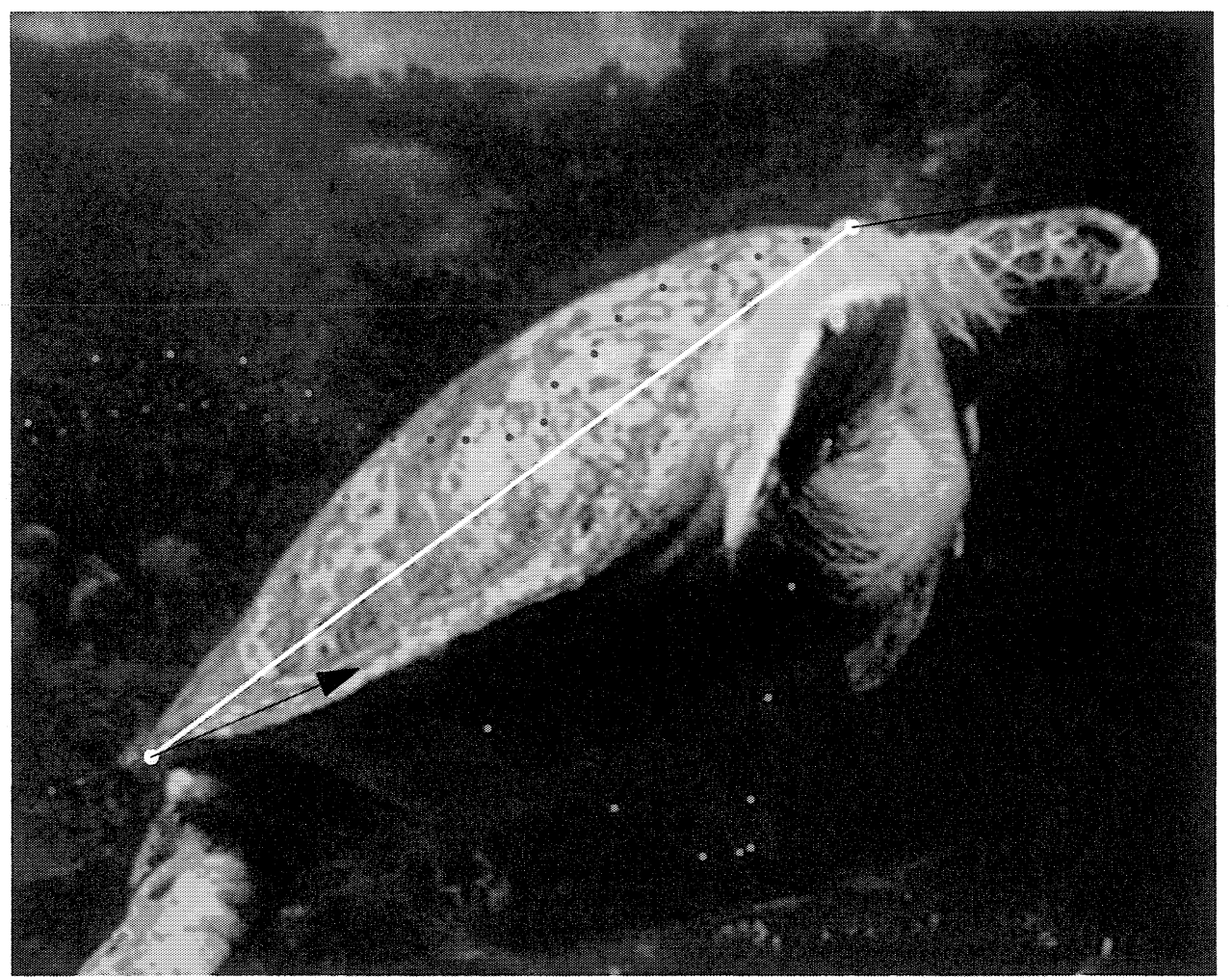

Figure 4-10: Representative frame with tracking data used to find steady swimming velocity. Velocity of extreme front and rear of carapace shown for comparison with body length indicated by white line.

To simultaneously record velocity and stroke frequency for three instances of forward swimming, frames were analyzed from two camera views of the events. A camera oriented perpendicular to an aquarium observation window was used to determine forward speed. As Myrtle moved into and out of the frame in less time than it took her to execute a full stroke, a camera in an underwater housing oriented perpendicular to the observation window was used to capture stroke frequency before, during and after the time period where her foils were visible from the side. The recorded limb downstroke and upstroke periods were found to be $1.6 \pm 0.5 \mathrm{~s}$, and $2.4 \pm 0.5 \mathrm{~s}$, with ratio of downstroke to upstroke period of $0.65 \pm 0.1$, for speeds of $0.25 \pm 0.05 \mathrm{BL} / \mathrm{s}$. A representative frame of the side view from from one instance of steady swimming is shown in Figure 4-10 with tracking dots shown for the extreme forward and extreme rear points on the shell, as well as the tip of the starboard foil. The distance between the two points on the shell is known, and is taken as the body length. The instan- 


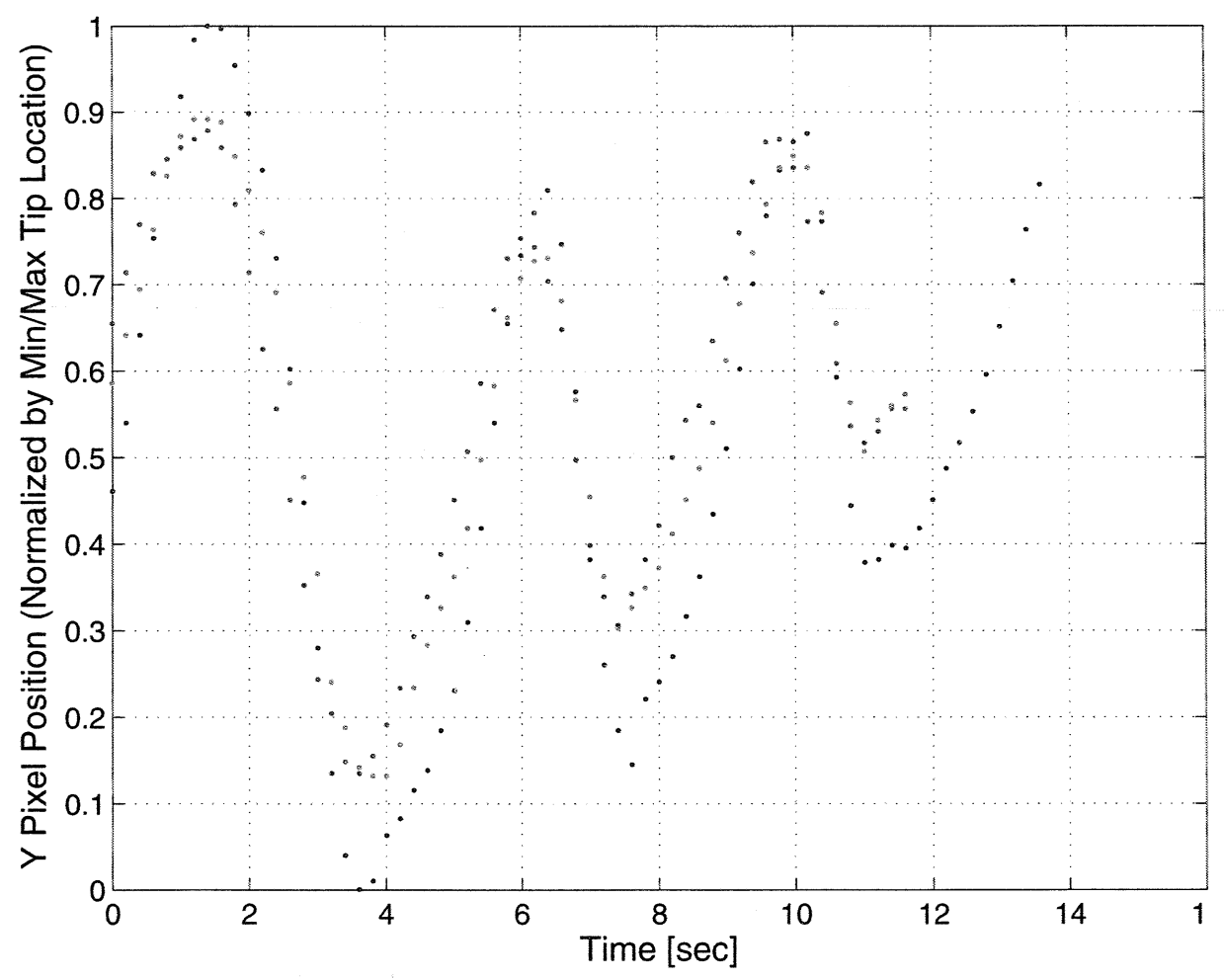

Figure 4-11: Tracking data for foil tip (blue), leading edge (green) and trailing edge (red) at join between upper and lower forelimb. Pixel data is used to determine downstroke and upstroke duration simultaneously with speed determination from Figure 4-10.

taneous velocities of the two points are depicted by the arrows in the figure. Mean velocity for this case is $0.24 \pm 0.05 B L / s$

While the speed is captured from the side view, the rear view (a frame of which is depicted in Figure 4-12) yields limb beat frequency over multiple cycles. In Figure 411, the y-pixel location of the foil tip, and of the intersection of the leading edge and trailing edge with the bend between the upper and lower limb sections, is plotted with respect to time, normalized such that the range extends from the maximum downward excursion of the tip at zero, to the maximum upward excursion at one. The downstroke and upstroke periods are taken to be the time between the extremes of the point on the leading edge of the foil. (Figure $4-12$ corresponds to $t=3.0$ seconds on Figure 4-11.) 


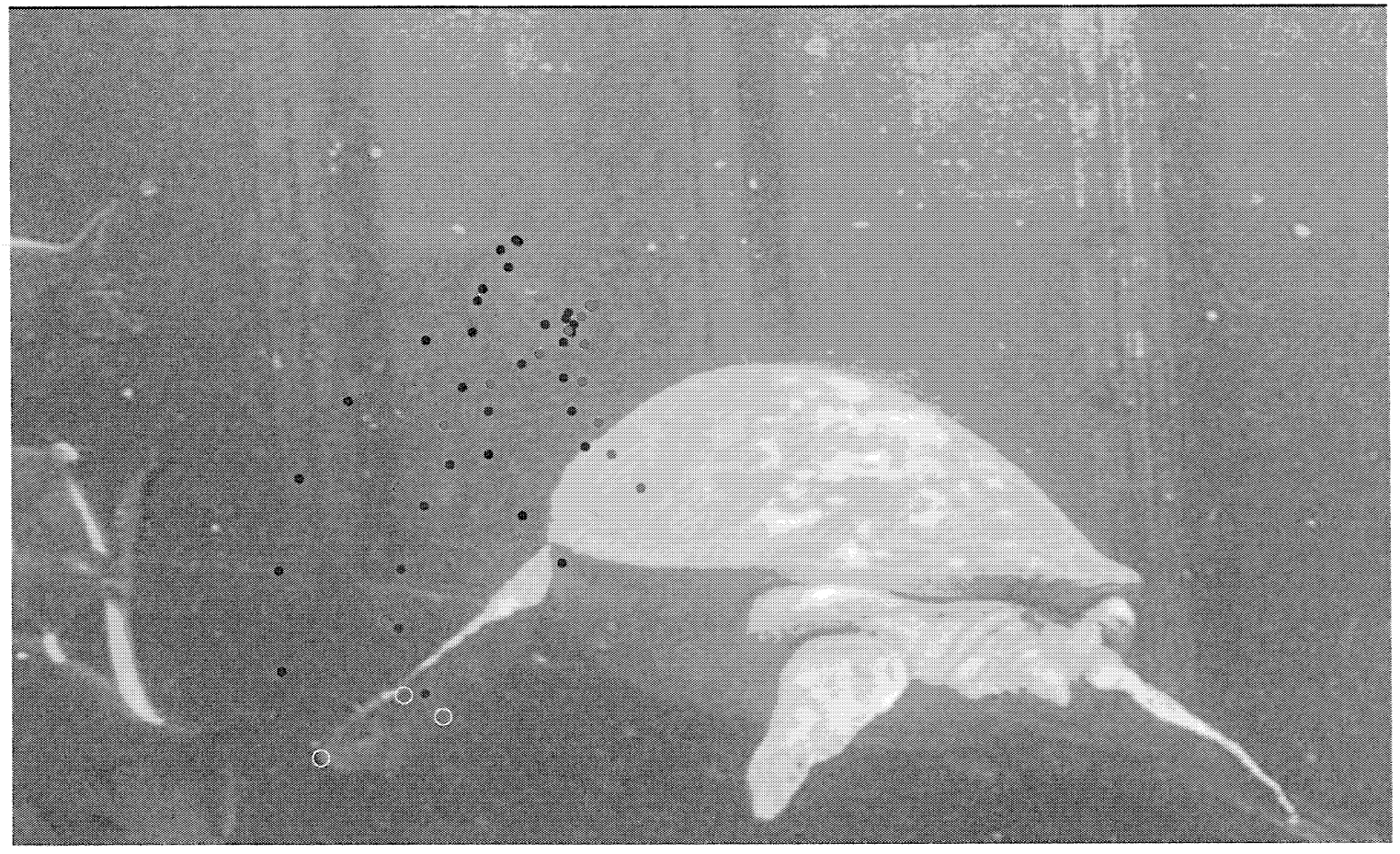

Figure 4-12: Representative frame with tracking data used to find steady swimming stroke durations. Points tracked are indicated on foil with white circles.

\subsection{Discussion of Results}

\subsubsection{Animal Motivation and Generalizability}

It is reasonable to assume that the maneuvers observed by Myrtle do not represent the outer limits of her performance. There was no immediate incentive to exert maximum effort in retrieving the food presented, as it was never withheld, regardless of the time taken for her to get to it. Indeed, from direct personal (and accidental) observation, sadly not captured on film, Myrtle's startle response appears to involve a much faster turn those discussed below. Certainly the maximum swimming speed of juvenile sea turtles is at least an order of magnitude higher than those observed in the Aquarium, with Davenport and Munks [33] recording speeds as high as 13BL/s, as compared to a maximum measured speed of $0.25 \mathrm{BL} / \mathrm{s}$.

All of the experiments involved a single specimen, one that has been in captivity for well over four decades, rendering it impossible to make statements about the 
generalizability of the results to even a single species, let alone sea turtles in general. However, for the purposes of informing engineering decisions about foil numbers, placement and capabilities, discussed at length in Chapter 5, even a single biological example of effective underactuated maneuvering strategies is very useful.

\subsubsection{Maneuvering Strokes}

While anterior-posterior motion was not seen in the steady swimming case, it played a significant role in all of the maneuvers observed. Where the primary power stroke mode was drag based, the anterior-posterior motion directly affected the direction of the force vector, i.e. the fore-aft motion during the power stroke directly opposed the desired surge force. The obvious advantage comes from the enhanced force vectoring capability. In the pitching up maneuver, where the forelimbs are pushed forward during the downstroke, the enhanced vectoring takes the form of greater braking power concurrent with the generation of the lift force creating the pitching moment. The result is a shorter turning radius for the pitching maneuver. Where the turtle needs to maintain more forward velocity, the extreme forward motion is curtailed, resulting is less braking motion with a more moderate pitching. In the case where Myrtle swims forward while attempting to increase depth, the freedom to move her limbs forward, rather than simply up and down, allows her to use a fore-aft rowing stroke to create surge impulse. The rowing stroke avoids the unwanted lift component that characterizes the asymmetric lift based stroke that she prefers during level swimming.

For the outside forelimb during the level turn, the power stroke thrust generation mode was lift based, with anterior motion preceding the downstroke, and posterior motion taking place during the downstroke. In other words, the limb was thrown forward in preparation for the downstroke, allowing the power stroke to start well in front of the head, and was then swept back well behind the median position by the end of the downstroke. Here, the fore-aft component not only had the effect of rotating the force vector within the horizontal plane, it also minimized the blade advance with respect to the water during the downstroke. This aspect of the outside forelimb stroke is covered in greater detail in Chapter 6, where the effect on force 
production is determined through experiments with a single foil, and the relationship between the use of the forward foil position and the limited upstroke torque capability of the turtle musculature is discussed.

\subsubsection{Steady Swimming}

Forelimb stroke frequency during dives for free swimming green turtles were reported by Hays [28] to range from $30-40$ beats per minute $(0.5-0.67 \mathrm{~Hz})$ at the beginning of a dive, changing to $10-14$ beats per minute $(0.17$ to $0.23 \mathrm{~Hz})$ during the latter part of the dive, ascribing the higher frequencies near the surface to the need to overcome initial positive buoyancy. During routine swimming, Myrtle typically swam at or slightly above the high range for the free swimming turtles during the negatively buoyant portion of their dives, tending to support the observation that Myrtle was negatively buoyant. [28] does not present data regarding the relative duration of upstroke and downstroke, although the role of the upstroke in generating thrust for subaqueous flight in birds and reptiles is an area of active research. Lovvorn [46] notes that estimates in the literature for upstroke thrust in swimming birds can range from zero to more than the downstroke thrust, and shows that swimming effort for a given mean speed increases as the variance of the speed increases, primarily because of the nonlinear increase in fuselage drag as a function of speed rather than increased inertial work.

The two available sources for detailed tracking of limb motion in sea turtles, Wyneken [75] and Davenport [33], use film analysis of hatchling and juvenile turtles, respectively, during routine forward swimming. The hatchlings are positively buoyant, while the juveniles are slightly negatively buoyant. All of the specimens massed at least three orders of magnitude less than Myrtle. In both cases, significant anterior/posterior motion was found during steady swimming, with the angle of motion relative to the body ranging from 20 to 30 degrees from the vertical. With the hatchlings the resulting motion of the fore limb tips with respect to the fluid is within 5 degrees of vertical, indicating that the blade advancement is minimized during the power stroke. In contrast, for Myrtle there was no significant anterior/posterior mo- 
tion found either during straight line acceleration up to, or regular swimming at, speeds of $0.25 \mathrm{BL} / \mathrm{s}$. There is great deal of room for further exploration into the effect of scaling and motivation in determining the preferred steady swimming stroke, as well as the range of individual and species variation.

\subsection{Conclusion}

Our observations of Myrtle the green sea turtle in Chapter 4 demonstrated that a rigid bodied animal using oscillating foils is capable of controlling attitude and heading at low speeds. In particular, detailed observations were made of the manner in which Myrtle executes level turns and controls her body pitch angle. Myrtle was capable of changing heading up to 180 degrees within three to four limb strokes, with turning rates up to $24^{\circ} / \mathrm{s}$ when swimming at speeds up to $0.25 \mathrm{BL} / \mathrm{s}$, primarily utilizing her forelimbs, and was able to pitch upwards in excess of 60 degrees within a single forelimb stroke, whether rising up to retrieve food or preparing to swim to the surface to breathe. While large angle roll motions were not observed, Myrtle was able to control her roll angle during these maneuvers, and during all other observed swimming behaviors. In other words, within the low speed regime where observations were possible, Myrtle admirably performed the basic set of maneuvers required by an agile underwater vehicle.

Based on analysis of the video, the forelimbs were used in both drag and lift modes, as passive control surfaces, and as fenders against solid objects, sometimes all within the space of a few seconds during a single maneuver. Significant anterior and posterior components were observed during the all maneuvers, with the direction and magnitude of this inline motion depending on the type of maneuver being performed. The hind limbs, in addition to being used as control surfaces at times, were also recruited as propulsors to initiate rapid yawing turns, and to accomplish fine positioning tasks, during which they executed highly 3D motions. In the following chapters, the use of the forelimbs during turning is replicated use the oscillating foil vehicle described in the previous chapters, and the effect of the inline (anterior-posterior) motion of the 
foils on force production is examined using a single foil in the laboratory. 


\section{Chapter 5}

\section{Biomimetic Foil Kinematics for Low Speed Maneuvering}

\subsection{Introduction}

The limb kinematics of swimming animals are constantly changing in response to external stimuli and animal state. In contrast, the maneuvering strategy employed to control Finnegan, described in Chapter 3, uses relatively small perturbations about constant harmonic oscillations to control attitude and heading, with no gross changes in the foil motion. While the constant motion simplifies the control algorithm, the one size fits all approach to actuation of vehicle maneuvers cannot be optimal for all maneuvers. Vehicle maneuvering performance can be improved by developing foil kinematics specifically for particular swimming actions; it is here that we can profitably look to biological examples for inspiriation. To this end, the turtle limb motions observed in Chapter 4 were adapted for use on the vehicle, with the goal of improving low speed maneuvering.

Our observations of Myrtle the green sea turtle in Chapter 4 demonstrated that a rigid bodied animal using oscillating foils is capable of controlling attitude and heading at low speeds. In particular, detailed observations were made of the manner in which Myrtle executes level turns and controls her body pitch angle. Myrtle was capable of changing heading up to 180 degrees within three to four limb strokes when 
swimming at speeds up to $0.25 \mathrm{BL} / \mathrm{s}$, primarily utilizing her forelimbs, and was able to pitch upwards in excess of 60 degrees within a single forelimb stroke, whether rising up to retrieve food or preparing to swim to the surface to breathe. While large angle roll motions were not observed, Myrtle was able to control her roll angle during these maneuvers, and during all other observed swimming behaviors. In other words, within the low speed regime where observations were possible, Myrtle admirably performed the basic set of maneuvers required by an agile underwater vehicle.

The limb kinematics from the turn pictured in Figures 4-6 and 4-7 were taken as a starting point for experimenting with level turns on the vehicle; a lift based stroke on the foil to the outside of the turn, using a highly feathered upstroke and thrust producing downstroke, was paired with a drag based stroke on the foil foil to the inside of the turn. To execute the drag based stroke as Myrtle does, with force vectored to produced a yaw moment, requires that the foil roll range extend well past vertical in the negative direction. In the actuator design used in the the testing described in Chatpers $2 \& 3$, foil roll action was limited to $\pm 85^{\circ}, 5^{\circ}$ shy of achieving vertical in either direction. The observations described in Chapter 4 led directly to a redesign the support bracket for the rotating cylinder which increased the foil roll range in the downward direction to $-120^{\circ}$. The extended roll range allows the tip of the foil to swing underneath the vehicle.

The limb kinematics were adapted as faithfully as possibly for Finnegan, and were successfully used to actuate $180^{\circ}$ heading changes in multiple trials. The sensitivity of the turning performance to perturbations in parameters such as roll and twist amplitude was shown to be low for those kinematics. We were then able to test the effect of two significant variations to the foil action. First, we executed the kinematics without using the inside foil to test the relative contribution to turning performance of the drag based inside forelimb stroke; the resulting reduction in turning rate and total turn angle allowed us to quantify the importance of the inside stroke for the turtle, and the benefit of the biomimetic actuator design changes for the the vehicle.

Second, we modified the roll and twist motions of the outside foil in order to quantify, for the first time on a free-swimming vehicle, the benefits of approximating . 
a sinusoidal nominal angle of attack for a lift-based thrust stroke. By following the biological example for the gross motions of the turn, and making the modifications on kinematics that already provided performance reasonably close to that of the turtle, we increase the confidence that this is a generalizable result, rather than a result specific to variations on a degenerate case.

\subsection{Adaptation of Turtle Level Turning Kinemat- ics}

The fidelity to the turtle limb kinematics of the foil motions performed by Finnegan was limited by three primary factors: no freedom to perform anterior-posterior motion, no joint along the foil span to replicate the (hand) joint of the turtle, and lack of quantitative measurements of limb twist angles. In adapting the forelimb motion to the forward foils, the anterior-posterior motion and spanwise joint were simply ignored. The roll angles and stroke frequencies were well characterized and the twist angles were selected based on a qualitative understanding of the limb twist for each half stroke, i.e. whether the limb was used in a drag based or lift based stroke, and whether the limb was highly feathered or presented to the flow with a high angle of attack.

In most case, Myrtle's use of her hind limbs during turns was limited to a single high amplitude drag based sweeping stroke to initiate the turn. This motion was generally completed before the fore limbs began stroking, after which the hind limbs were both held stationary, the outside paddle close to the body and the inside paddle straight out toward the inside of the turn. (See Figure 5-1 for a cartoon schematics of this motion.) The rear foils on Finnegan were incapable of reproducing this turn initiating stroke, but as the hind limbs were held stationary for the remainder of the turn, there is justification for bringing the rear foils of the vehicle to a fixed position, then holding them stationary, and treating the discrepancy in initial foil motion primarily as a difference in initial conditions. 


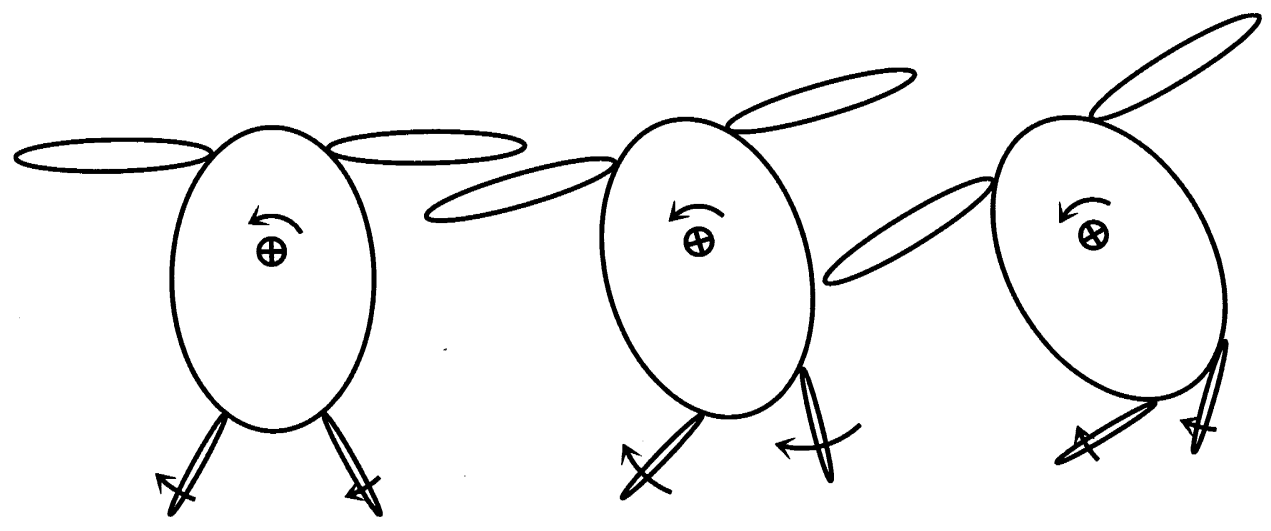

Figure 5-1: Cartoon showing top view of turtle hindlimb kinematics during kick start of level turning. The motion of the hindlimbs depicted here is not available to the vehicle foils, which are instead held stationary throughout the turn.

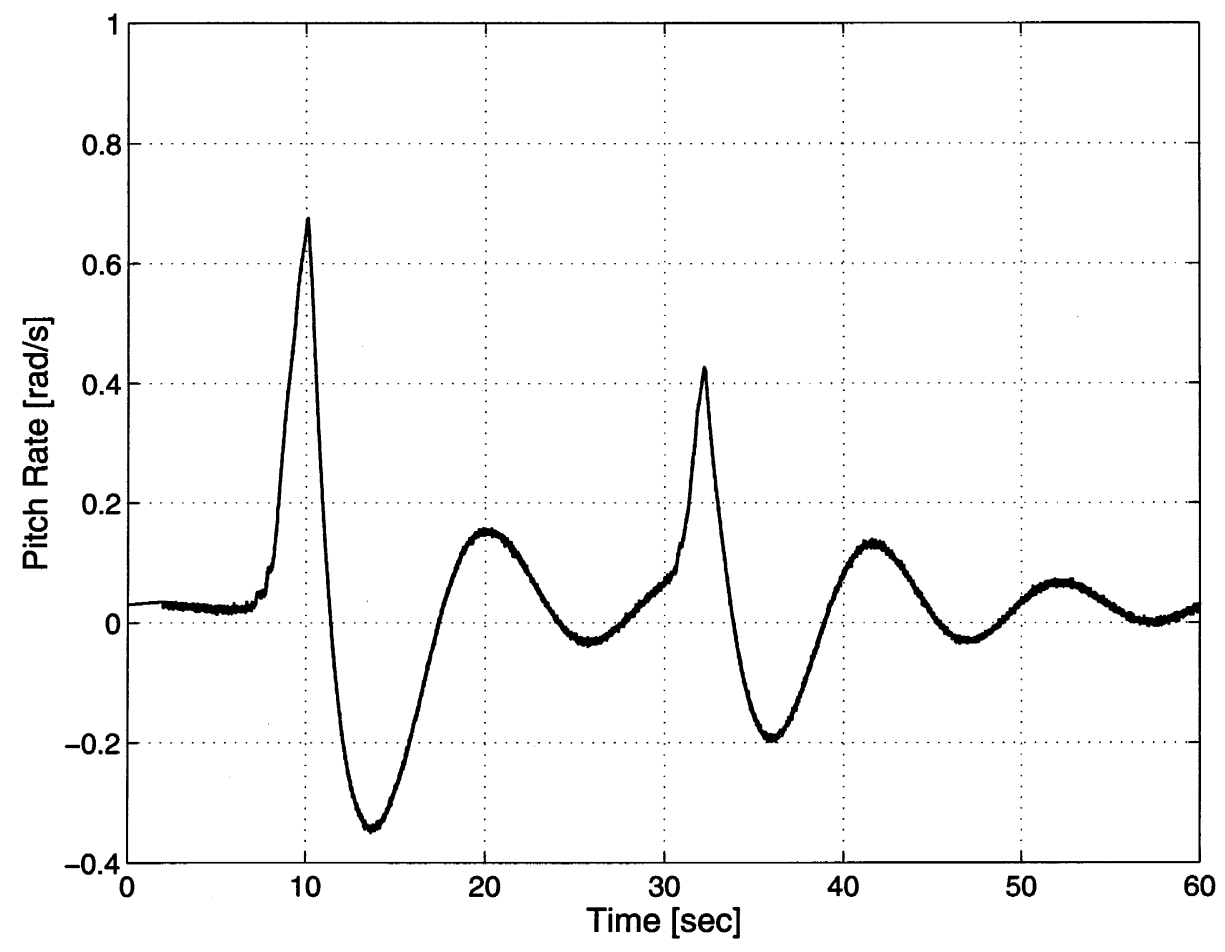

Figure 5-2: Vehicle unactuated roll rate response, in stable trim configuration, to repeated disturbance in roll. 


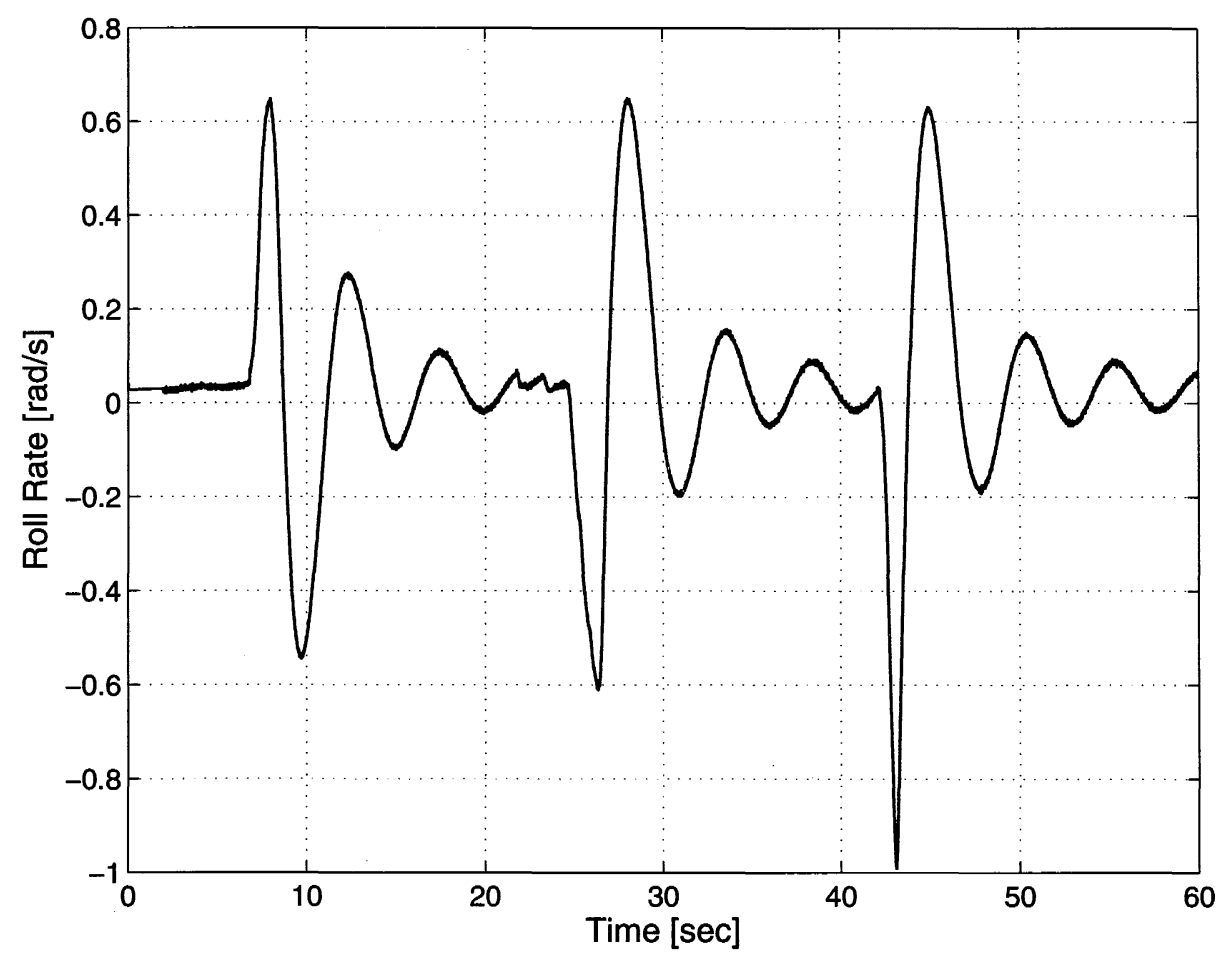

Figure 5-3: Vehicle unactuated pitch rate response, in stable trim configuration, to repeated disturbance in pitch.

Each trial was begun with the vehicle accelerating to a level cruising speed of $0.6 \pm 0.05 \mathrm{~m} / \mathrm{s}(0.46 \pm 0.04 \mathrm{BL} / \mathrm{S})$ while controlling body roll and pitch angle, using the control strategy detailed in Chapter 3. Once steady cruising velocity was achieved, the vehicle continued the foil motion until all four foils (simultaneously) reached the maximum roll excursion of 60 degrees, at which point the rear foils were smoothly brought down to 0 degrees roll and 0 degrees twist within $0.5 \mathrm{sec}$ and held for the remainder of the trial. Experiments were performed with the vehicle in stable trim configuration. The vehicle passive response to disturbances in body roll and pitch while in the stable trim configuration are damped oscillations with natural frequencies of $0.18 \mathrm{~Hz}$ and $0.14 \mathrm{~Hz}$ respectively, as calculated from the free response to pure roll (Figure 5-2) and pure pitch (Figure 5-2) disturbance. For these trials, the foils were stationary, held out horizontally from the body. 

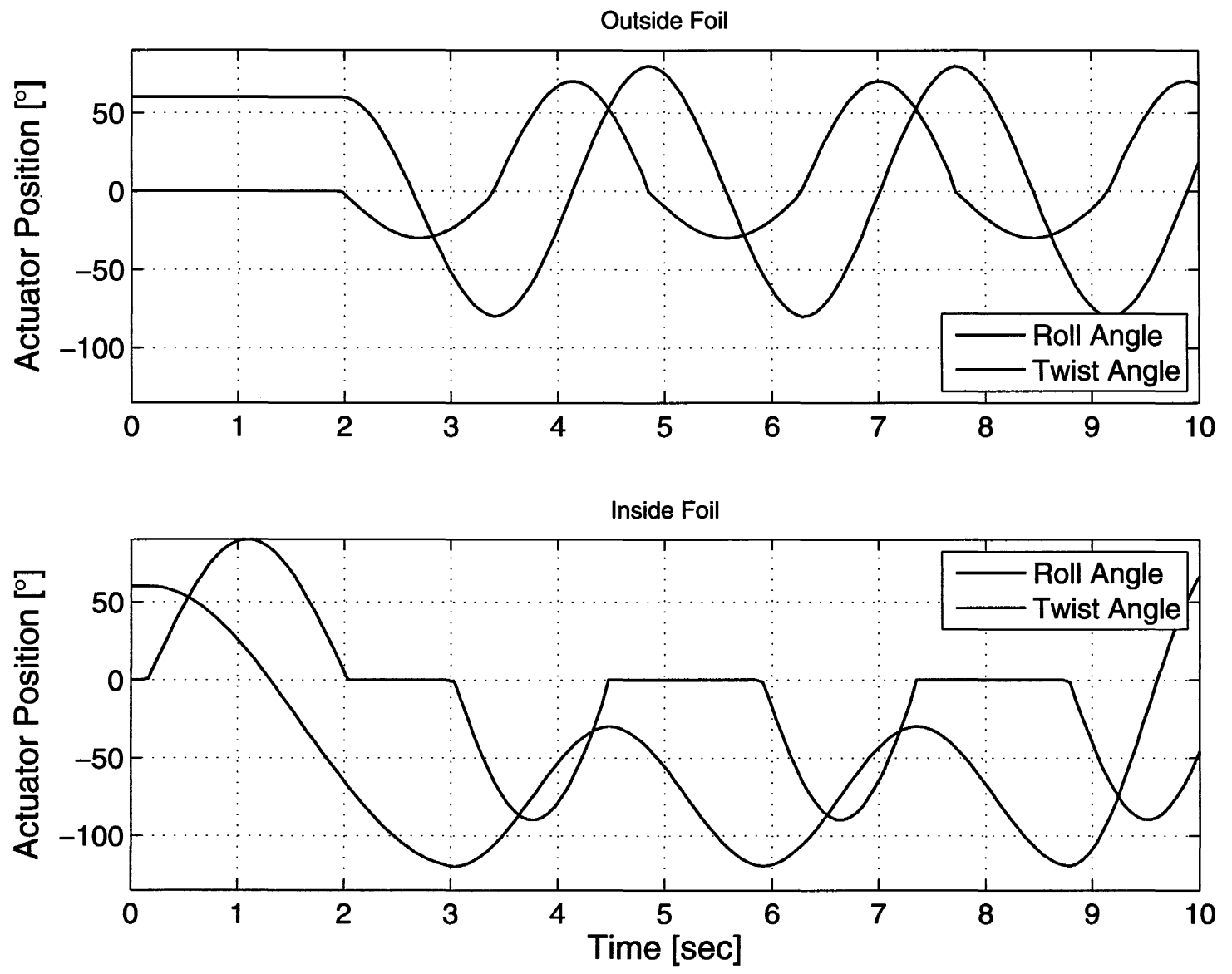

Figure 5-4: Foil kinematics during biomimetic level turn, for forward foils to the inside and outside of the turn, using combined forelimb motion with piecewise sinusoidal motion. Case $\mathbf{B}$ 

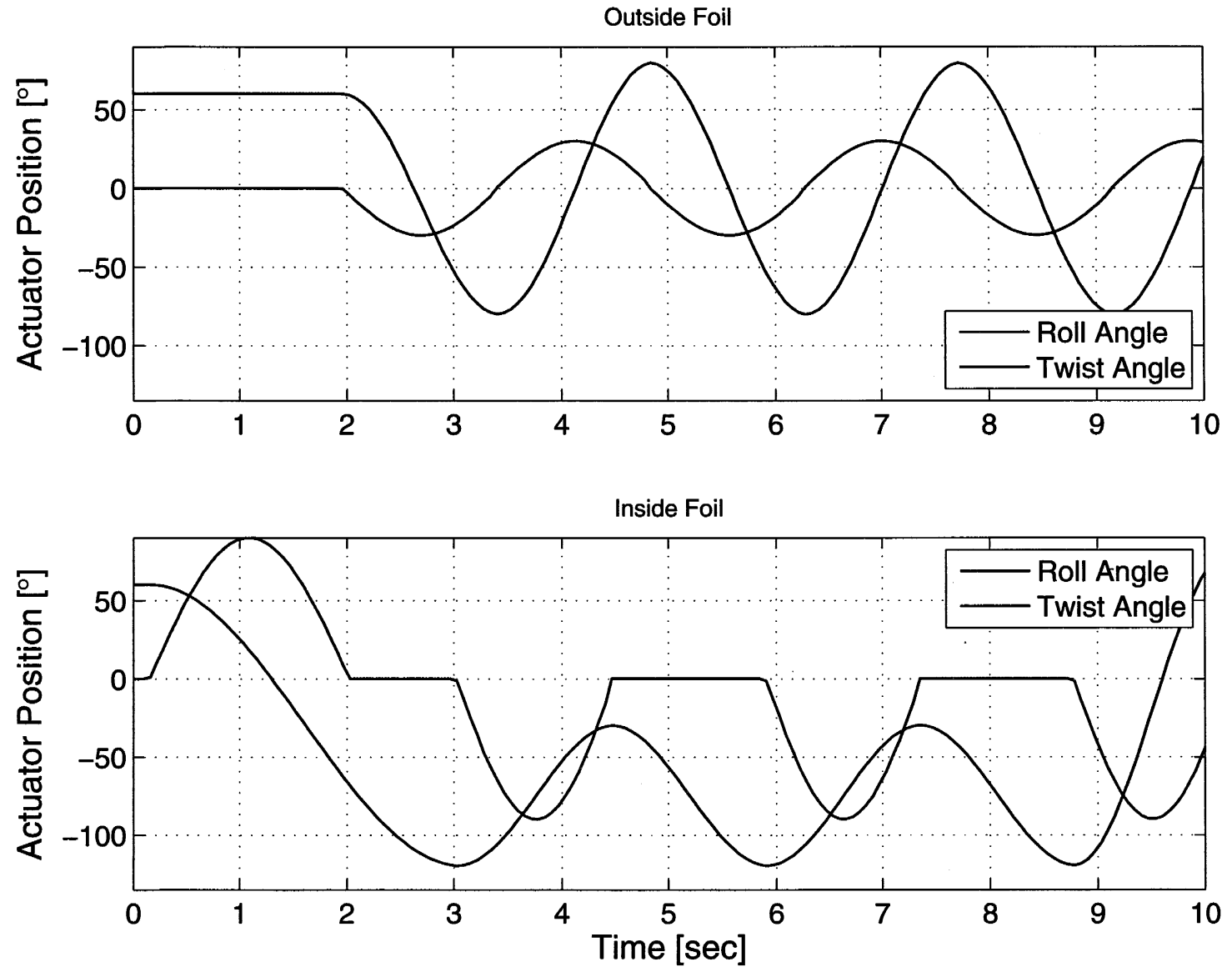

Figure 5-5: Foil kinematics during biomimetic level turn, for forward foils to the inside and outside of the turn, using combined forelimb motion. Outside forelimb motion altered for symmetric twist. Case B' 

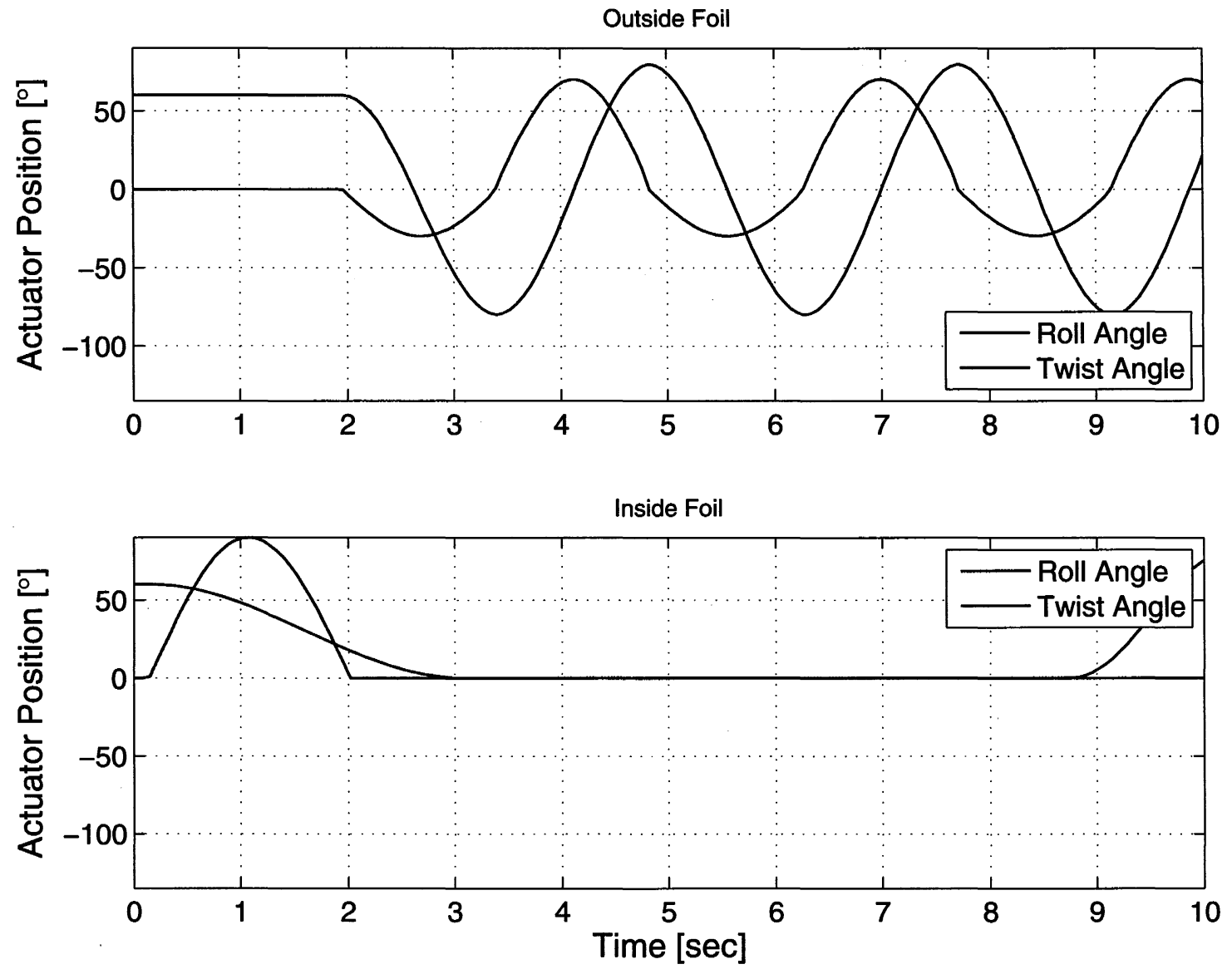

Figure 5-6: Foil kinematics during biomimetic level turn, for forward foils to the inside and outside of the turn, using outside forelimb action only, and piecewise sinusoidal motion. Case A 

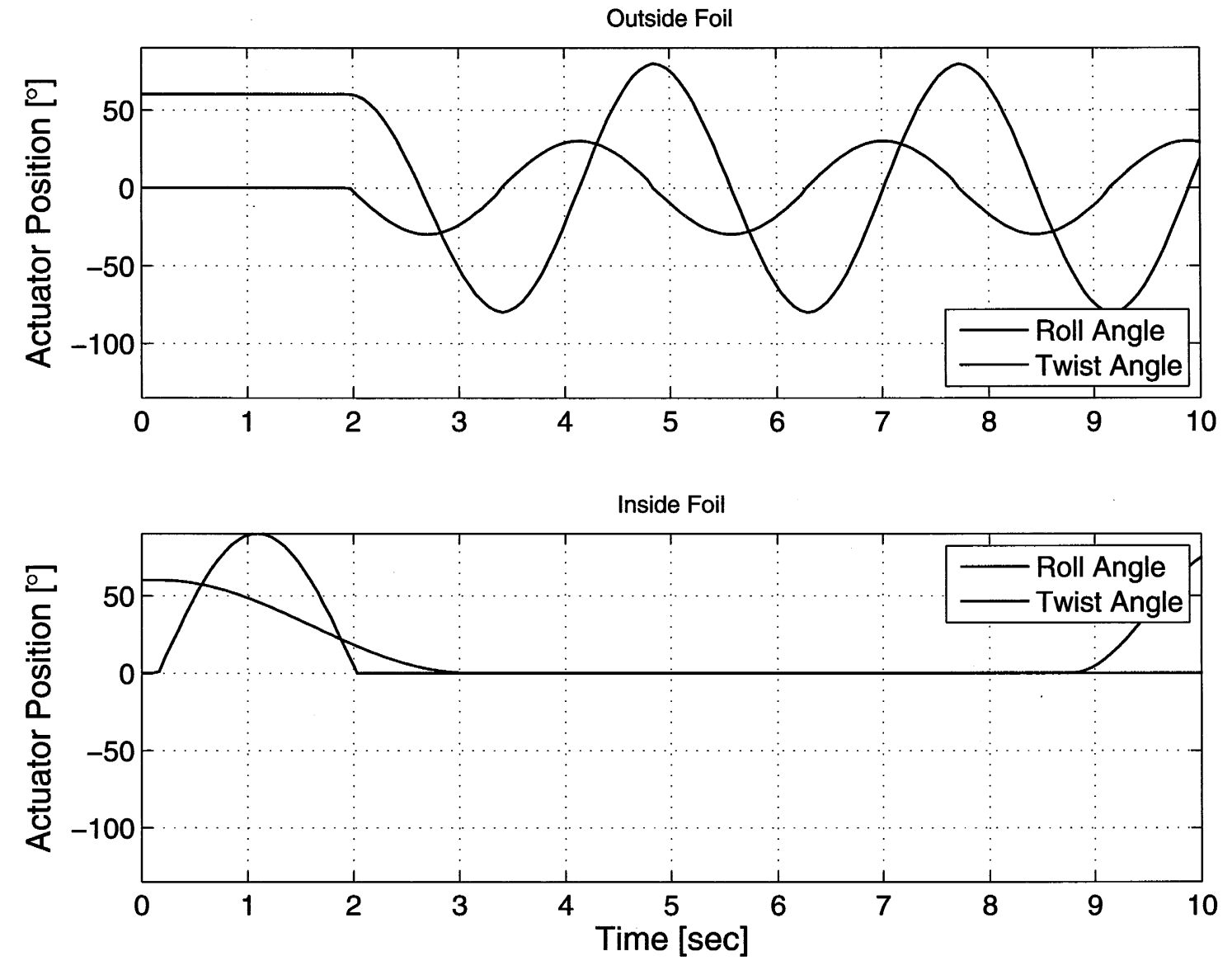

Figure 5-7: Foil kinematics during biomimetic level turn, for forward foils to the inside and outside of the turn, using outside forelimb action only. Outside forelimb motion altered for symmetric twist. Case $\mathbf{A}^{\prime}$ 

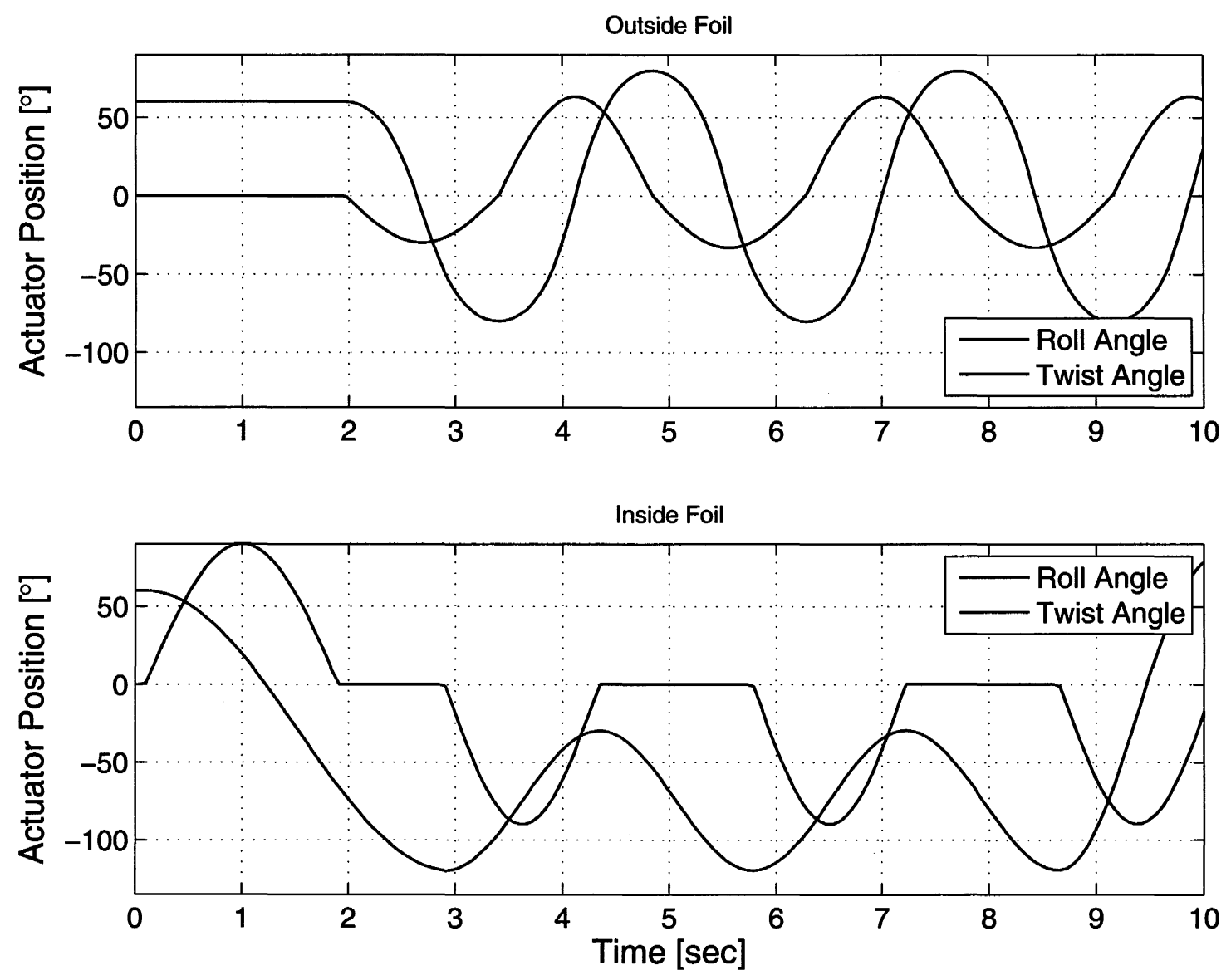

Figure 5-8: Foil kinematics during biomimetic level turn, for forward foils to the inside and outside of the turn, using combined forelimb action, with piecewise sinusoidal $\alpha(t)$ for the outside foil. Case $\mathbf{C}$ 


\subsubsection{Combined Lift and Drag Foil Actions.}

The baseline kinematics for the combined use of the two forward foils were composed of piecewise sinusoidal trajectories in the forward foil roll and twist angles, as shown in Figure 5-4. The foils start in a raised position with roll angle of $60^{\circ}$ and zero twist angle. After a transition period $(0 s<t<3.5 s)$, the kinematics are described by:

Inside Foil: Drag Based Kinematics

$$
\begin{aligned}
& \phi^{I}(t)=\phi_{\mathbf{0}}^{\mathbf{I}} \cos \left(2 \pi f t+\psi^{I}\right)-\left(\phi_{\text {max }}^{-}-\phi_{\mathbf{0}}^{\mathbf{I}}\right) \\
& \theta^{I}(t)=\left\{\begin{array}{cc}
\frac{\pi}{2} \sin \left(2 \pi f t+\psi^{I}\right), & \dot{\phi}(t)>0 \\
0, & \dot{\phi}(t)<0
\end{array}\right.
\end{aligned}
$$

Variables : $\phi_{0}^{\mathbf{I}}$

Outside Foil: Lift Based Kinematics

$$
\begin{aligned}
& \phi^{o}(t)=\phi_{0}^{o} \cos \left(2 \pi f t+\psi^{o}\right) \\
& \theta^{o}(t)= \begin{cases}\theta_{0}^{o, U} \sin \left(2 \pi f t+\psi^{o}\right), & \dot{\phi}(t)>0 \\
\theta_{0}^{o, D} \sin \left(2 \pi f t+\psi^{o}\right), & \dot{\phi}(t)<0\end{cases}
\end{aligned}
$$

Variables : $\theta_{\mathbf{0}}^{\mathbf{o}, \mathbf{U}}, \theta_{\mathbf{0}}^{\mathbf{o}, \mathbf{D}}$

$$
\begin{aligned}
\phi_{0}^{o} & =85^{\circ} \\
\text { Constants : } \phi_{\max }^{-} & =-120^{\circ} \\
\psi^{I}-\psi^{o} & =\pi / 3 \\
f & =1 / 3 \mathrm{~Hz}
\end{aligned}
$$

The inside foil begins the motion with a downstroke swinging from the positive extreme of $60^{\circ}$ to the negative extreme, $\phi_{\max }^{-}=120^{\circ}$, where the tip of the foil is underneath the vehicle. To avoid generating an excessive body roll moment before the foil gets below the horizontal, the foil is feathered during the first part of the downstroke (piecewise sinusoidal twist amplitude of $90^{\circ}$ for $t<2$ in Figure 5-4b). 
The twist angle is brought back to zero for the last part of the transition downstroke $(2<t<3)$ and the foil beings to generate a body yaw moment. For the remainder of the maneuver, the inside foil repeats a rowing stroke to generate a yaw moment, presenting the broad side of the foil during the downstroke (twist angle held at zero,) and feathering the foil completely during the upstroke (twist angle trajectory a half period of a sinusoid.) The roll amplitude, $\phi_{0}^{I}$ was varied during testing as described below, and affects the roll bias according to $\phi_{\max }^{-}-\phi_{0}^{I}$, so as to take full advantage of the foil roll range.

The outside foil remains stationary during the first part of the transition period to allow for a phase lag between the inside foil and the outside foil of $\psi_{I}-\psi_{o}=\pi / 6$, mimicking the typical lag between limbs for the turning turtle. Roll is sinusoidal with constant amplitude and period except for the first downstroke, which is slightly different to account for the difference between the desired amplitude, $\phi_{0}^{o}=85^{\circ}$, and initial position, $60^{\circ}$, as seen where $2.0 s<t<3.5 s$ in Figure 5-4a. Twist is piecewise sinusoidal after $t=2.0 \mathrm{~s}$, with the amplitude of the upstroke and downstroke twist independently varied during testing as described below.

Compare the stroke period and foil to foil lead time with the position tracking data for the referenced turn shown in Figure 4-8. The normalized vertical position of the inside and outside limb tips, and of the leading and trailing edge at the joint between upper and lower limb sections, were found using manual selection of pixel position on a sequence of digital video frames. The position of leading edge join between upper and lower limb was used to determine phase difference and period, rather than the position of the tip, which lags the rest of the limb more at the end of the downstroke than the end of the upstroke.

The vehicle performance against which all other results are compared in Table 5.1 was recorded ( $n=5)$ with a roll amplitude on the inside foil of $\phi_{0}^{I}=45^{\circ}$, a downstroke twist amplitude of $\theta_{0}^{o, D}=30^{\circ}$ on the outside foil, and upstroke twist amplitude of $\theta_{0}^{o, U}=70^{\circ}$. The resulting kinematics on the outside foil mimic the high angle of attack downstroke and highly feathered upstroke observed for the turtle outside forelimb in Chapter 4. These kinematics are referred to throughout as Case B. 
Sea turtles limbs at all development stages can produce much more torque in the down stroke than the upstroke, as discussed in more detail in Chapter 6. The vehicle foils, in contrast, are actuated in such a way that there is no difference between upstroke and downstroke output power capacity. While Myrtle consistently demonstrates a preference for highly feathered upstrokes, there is no inherent need to restrict the vehicle to the use of the upstroke as a recover stroke in anticipation of a downward power stroke. To take advantage of the ability to perform the upstroke with the same power as the downstroke, the yawing maneuver was repeated, this time with the same twist magnitude on the upstroke and downstroke. In this way the effect on turning performance of the turtle's need to use the upstroke as a recovery stroke, rather than an additional power stroke, was examined. Two amplitudes were tested, with $\theta_{0}^{o, U}=\theta_{0}^{o, D}=30^{\circ}$ for Case B' and $\theta_{0}^{o, U}=\theta_{0}^{o, D}=40^{\circ}$ for Case B"

The sensitivity to the roll amplitude of the inside foil was tested by setting $\phi_{0}^{I}=30^{\circ}$ (resulting in a roll bias of exactly $-90^{\circ}$ ) for Case $\mathbf{B}^{*} . \theta_{0}^{o, D}=30^{\circ}$ and $\theta_{0}^{o, U}=70^{\circ}$ were used on the outside foil. $n=5$ for all sets of kinematics described in this chapter.

\subsubsection{Lift Based (Outside) Foil Action Alone.}

To determine the relative contribution of the inside foil to the actuation of the turn, the kinematics of the inside foil were modified to hold the foil stationary in the horizontal position, i.e. $\phi=0$ and $\theta=0$, throughout the turn. The resulting roll and twist angles for both foils are shown in Figure 5-6, and described by: 
Stationary Inside Foil

$$
\begin{aligned}
\phi^{I}(t) & =0 \\
\theta^{I}(t) & =0
\end{aligned}
$$

Variables : none

Outside Foil: Lift Based Kinematics

$$
\begin{aligned}
& \phi^{o}(t)=\phi_{0}^{o} \cos \left(2 \pi f t+\psi^{o}\right) \\
& \theta^{o}(t)= \begin{cases}\theta_{0}^{o, U} \sin \left(2 \pi f t+\psi^{o}\right), & \dot{\phi}(t)>0 \\
\theta_{0}^{o, D} \sin \left(2 \pi f t+\psi^{o}\right), & \dot{\phi}(t)<0\end{cases} \\
& \text { Variables : } \theta_{\mathbf{0}}^{\mathbf{o}, \mathbf{U}}, \theta_{\mathbf{0}}^{\mathbf{o}, \mathbf{D}}
\end{aligned}
$$

The inside foil performs a single downstroke, highly feathered, over the first 3 seconds to bring the foil to the horizontal position, and stops. The outside foil kinematics are identical to those described for the baseline kinematics. The beginning of the outside foil motion coincides with end of the inside foil twist motion. For a visual comparison between the two sets of kinematics, see Figure 5-9 and Figure 5-10, showing the foil position with respect to the body for the combined foil action and the single foil action, respectively. The foil action through to the beginning of the first outside foil upstroke is depicted.

For direct comparison to the baseline kinematics of Case $\mathbf{B}$, the same twist amplitudes for the downstroke and upstroke, $\theta_{0}^{o, D}=30^{\circ}$ and $\theta_{0}^{o, U}=70^{\circ}$, were used in Case A. In addition, as in the combined foil case, the effect of using a high angle of attack stroke for both the upstroke and the downstroke was tested, with $\theta_{0}^{o, U}=\theta_{0}^{o, D}=30^{\circ}$ for Case $\mathbf{A}^{\prime}$ 


\subsubsection{Combined Foil Action with Sinusoidal $\alpha(t)$.}

[27] and [55] document the importance of the shape of the angle of attack trace in thrust production with 2D foils. Significant improvements in efficiency and mean thrust are documented throughout the parameter space tested when the foil kinematics are altered from simple sinusoidal motions to motions which produce angle of attack profiles more closely resembling sinusoids. The gains are particularly sharp in those parts of the parameter space with high Strouhal number and low $\alpha_{\max }$, where the angle of attack profiles are degenerate in the sense that there are multiple maxima and minima throughout a single period of motion. Where a degenerate angle of attack profile is "repaired" by replacing it with a single peaked angle of attack profle, there is a significant difference in the wake structure observed behind the foil as well.

An analog of this nominal angle of attack can be calculated for a three dimensional foil (as discussed in Chapter 2) through the relationship:

$$
\alpha(t)=-\arctan \left(\frac{\hat{\dot{\phi}}(t) * r_{0.7}}{U}\right)+\hat{\theta}(t)
$$

This relationship defines the angle of attack as it is calculated at $r_{0.7}$, a point $70 \%$ down the span from the root to the tip of the foil, following the convention for propellor calculations and previous work with rolling and twisting foils [20], [53]. To calculate angle of attack, knowledge of both the foil kinematics and the flow impinging on the foil is required.

The angle of the flow with respect to each foil on the vehicle can be approximated as a function of the vehicle velocity, $\dot{\bar{U}}$, the rigid rotation rate of the vehicle, $\bar{\omega}$, and the foil roll angular rate $\dot{\theta}$, as well as the vehicle and foil geometry. The angle of attack is then moderated by the twist position. An important caveat here is that this calculation of nominal angle of attack is based on the assumption of totally uniform fluid flow. As in all the single foil experimental work described, there is flow induced on the foil by the foil wake. Further, when the foil is attached to a vehicle, there are likely to be significant body wake effects, which do not enter the nominal angle of 
attack calculations.

To test the effectiveness of approximating a sinusoidal angle of attack for the lift based thrusting stroke of the outside foil, the kinematics of the outside foil were modified again. The initial downstroke of the outside foil was identical to that described for the baseline foil action, but the kinematics for the remaining foil strokes were calculated to satisfy:

Inside Foil: Drag Based Kinematics

$$
\begin{gathered}
\phi^{I}(t)=\phi_{\mathbf{0}}^{\mathbf{I}} \cos \left(2 \pi f t+\psi^{I}\right)-\left(\phi_{\max }^{-}-\phi_{\mathbf{0}}^{\mathbf{I}}\right) \\
\theta^{I}(t)=\left\{\begin{array}{cc}
\frac{\pi}{2} \sin \left(2 \pi f t+\psi^{I}\right), & \dot{\phi}(t)>0 \\
0, & \dot{\phi}(t)<0
\end{array}\right.
\end{gathered}
$$

Variables : $\phi_{0}^{\mathbf{I}}$

Outside Foil: Sinusoidal Angle of Attack

$$
\begin{array}{r}
\alpha(t)= \begin{cases}\alpha_{\text {max }}^{D} \sin \left(2 \pi f t+\psi^{o}\right), & \dot{\phi}<0 \\
\alpha_{\text {max }}^{U} \sin \left(2 \pi f t+\psi^{o}\right), & \dot{\phi}>0\end{cases} \\
\theta^{o}(t)= \begin{cases}\theta_{0}^{o, D} \sin \left(2 \pi f t+\psi^{o}\right), & \dot{\phi}<0 \\
\theta_{0}^{o, U} \sin \left(2 \pi f t+\psi^{o}\right), & \dot{\phi}>0\end{cases} \\
\phi_{\text {max }}=\phi_{0}^{o} \\
\text { Variables : } \alpha_{\text {max }}^{\mathbf{U}}, \alpha_{\text {max }}^{\mathbf{D}} \\
\text { Constants : } \phi_{\text {max }}^{-}=-120^{\circ} \\
\psi^{I}-\psi^{o}=\pi / 3
\end{array}
$$

Given some constant vehicle speed, $U$, the kinematics are fully determined by $\alpha_{\max }, \phi_{0}$, and the form (piecewise sinusoidal) of $\theta(t)$ and $\alpha(t)$ : 


$$
\begin{gathered}
\theta_{0}^{D}=f\left(\alpha_{\max }^{D}, \phi_{0}^{o}\right) \\
\theta_{0}^{U}=f\left(\alpha_{\max }^{U}, \phi_{0}^{o}\right) \\
\phi(t)=f(\alpha(t), \theta(t))
\end{gathered}
$$

An iterative process is used to calculate $\theta_{0}^{D}, \theta_{0}^{U}$, and $\phi(t)$. Begin by setting $\hat{\theta_{0}}=0$, and calculate the resulting $\hat{\dot{\phi}}(t)$ throughout the halfstroke from the geometric relationship defining $\alpha(t)$ given in 5.20 .

We can now numerically integrate to find the amplitude of the roll motion for the halfstroke:

$$
\hat{\phi}_{0}=\int_{0}^{\frac{T}{2}} \hat{\dot{\phi}}(t) d t
$$

$\hat{\phi}_{0}$ is a function of the only free variable, $\hat{\theta}_{0}$, hence we can iteratively solve for $\hat{\theta}_{0}=\theta_{0}$ by requiring $\hat{\phi}_{0}-\phi_{0}=0$. Roll as a function of time is generated from $U=$ $0.5 \mathrm{~m} / \mathrm{s}, \phi(t)=\int_{0}^{t} \dot{\phi(t)} d t$. The resulting $\theta(t)$ and $\phi(t)$ are illustrated for $\alpha_{\max }^{D}=40^{\circ}$ and $\alpha_{\max }^{U}=10^{\circ}$ in Figure 5-8. (The inside foil kinematics, also shown in Figure 5-8 are the same as described for the baseline combined foil test.)

Intuitively, the algorithm starts with a twist amplitude so low that the foil barely needs to be moving to reach the desired angle of attack through the downstroke. Since the foil is not moving very fast, by the end of the downstroke the roll position has only changed a few degrees. Increase the twist amplitude, and the foil needs to move faster to achieve the desired angle of attack, simply because the foil is more feathered. Eventually, as the twist amplitude is increased, the foil velocities are high enough that the foil travels the desired distance in roll during the downstroke. The iteration can be performed efficiently because the roll angle traversed by the foil increases monotonically as the twist amplitude increases from zero.

Using the results from Case B as described below, values of $\alpha_{\max }^{D}=40^{\circ}$ and 


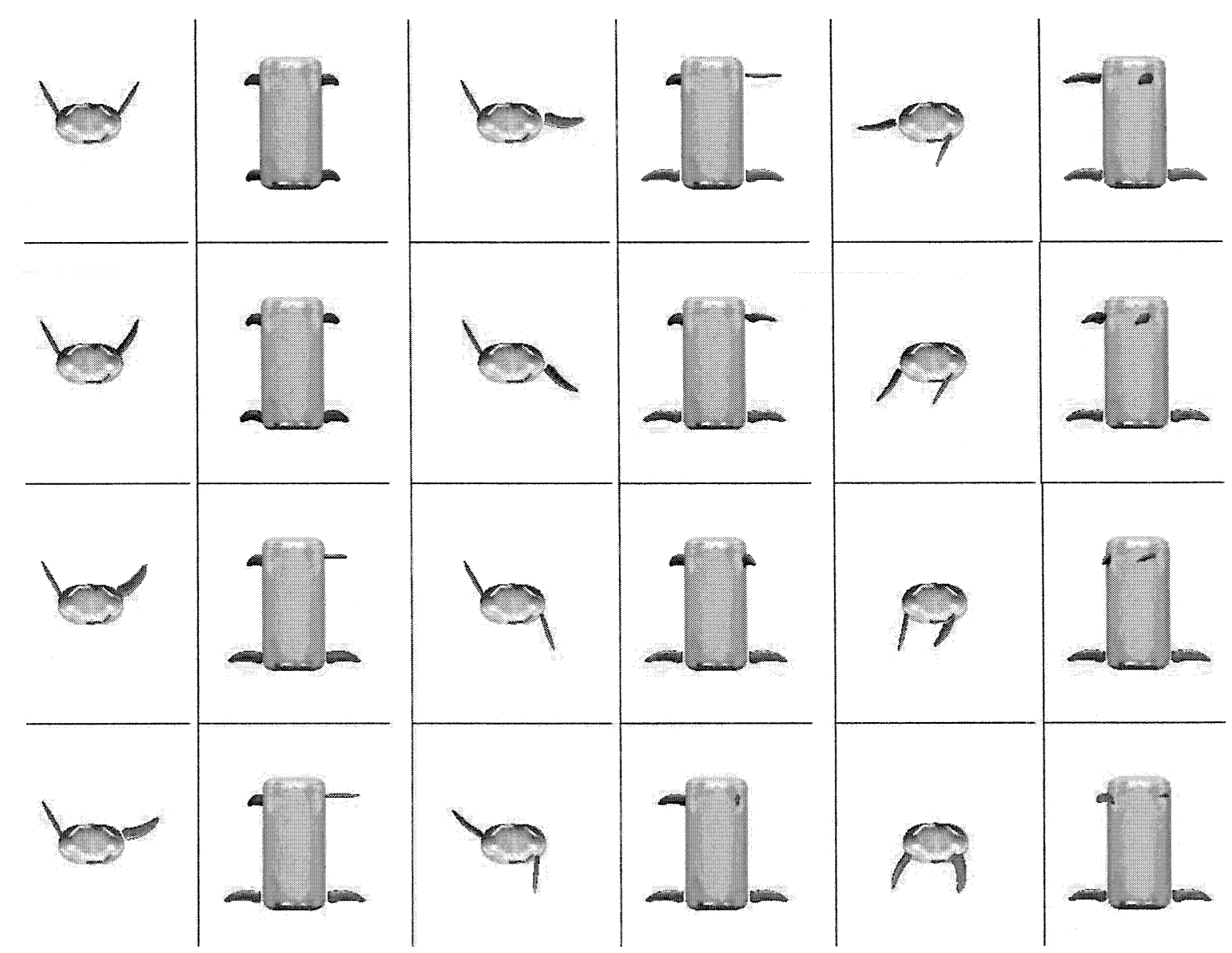

Figure 5-9: Visualization of foil kinematics from start of biomimetic level turn using combined forelimb action.. Top and bottom views are paired at 0.33 second intervals. Case $\mathbf{B}$

$\alpha_{m a x}^{U}=10^{\circ}$ where chosen for Case $\mathbf{C}$ (along with $\phi_{0}^{I}=45^{\circ}$ ) to allow direct comparison to Case B. In addition, sensitivity to the downstroke angle of attack was tested by setting $\alpha_{\max }^{D}=30^{\circ}$ in Case $\mathbf{C}^{*}$.

\subsection{Results}

\subsubsection{Combined Lift and Drag Foil Actions.}

A detailed analysis of the results for the baseline kinematics, Case $\mathbf{B}$, is presented here. Mean heading and mean heading rate for this case are presented in Figure 514 and Figure 5-15 respectively, and compared to those for Case $\mathbf{A}$ and Case $\mathbf{C .}$ Zero heading corresponds to the vehicle heading angle at $\mathrm{t}=0 \mathrm{sec}$ in order to allow 


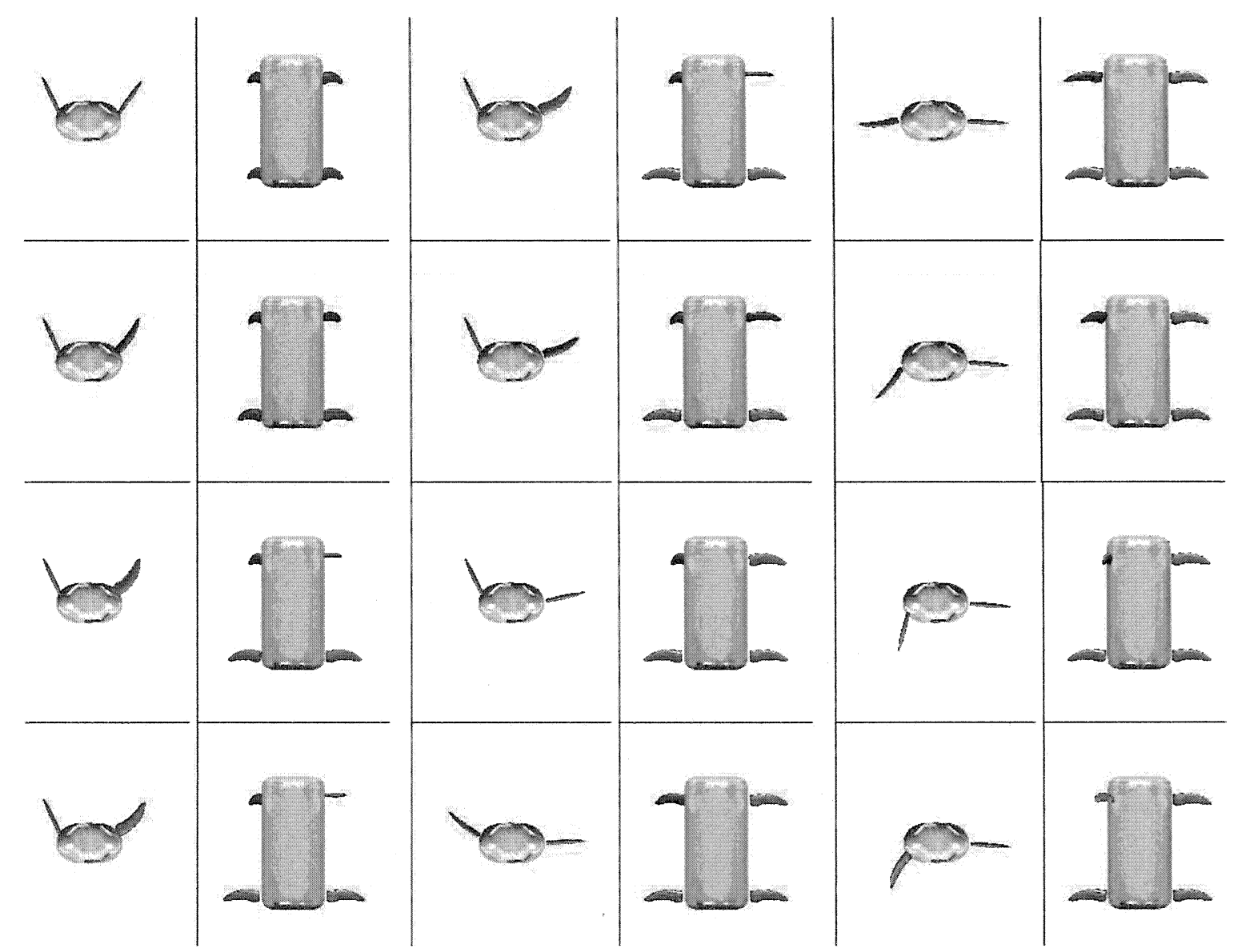

Figure 5-10: Visualization of foil kinematics from start of biomimetic level turn using outside forelimb action only. Top and bottom views are paired at 0.33 second intervals. Case $\mathbf{A}$

comparison between trials. For a direct visual comparison between the vehicle motion in this case and the model turn by Myrtle, see Figure 5-12 and Figure 4-7. The cartoon renderings in Figure 5-11 and in Figure 5-12 accurately reflect the vehicle body position and attitude. The virtual camera position for Figure 5-12 is fixed to approximate the relative camera position with respect to the turtle in Figure 4-7. The time step between frames is identical for both Figure 5-12 and Figure 4-7.

There is a transition period of two seconds from the end of steady swimming to the beginning in earnest of the maneuvering kinematics, during which the outside foil is stationary waiting for the inside foil to reach the correct position and phase. The large variance for body attitude during this part of the maneuver, for this and other kinematics, results from the relatively large variance in initial attitude conditions 


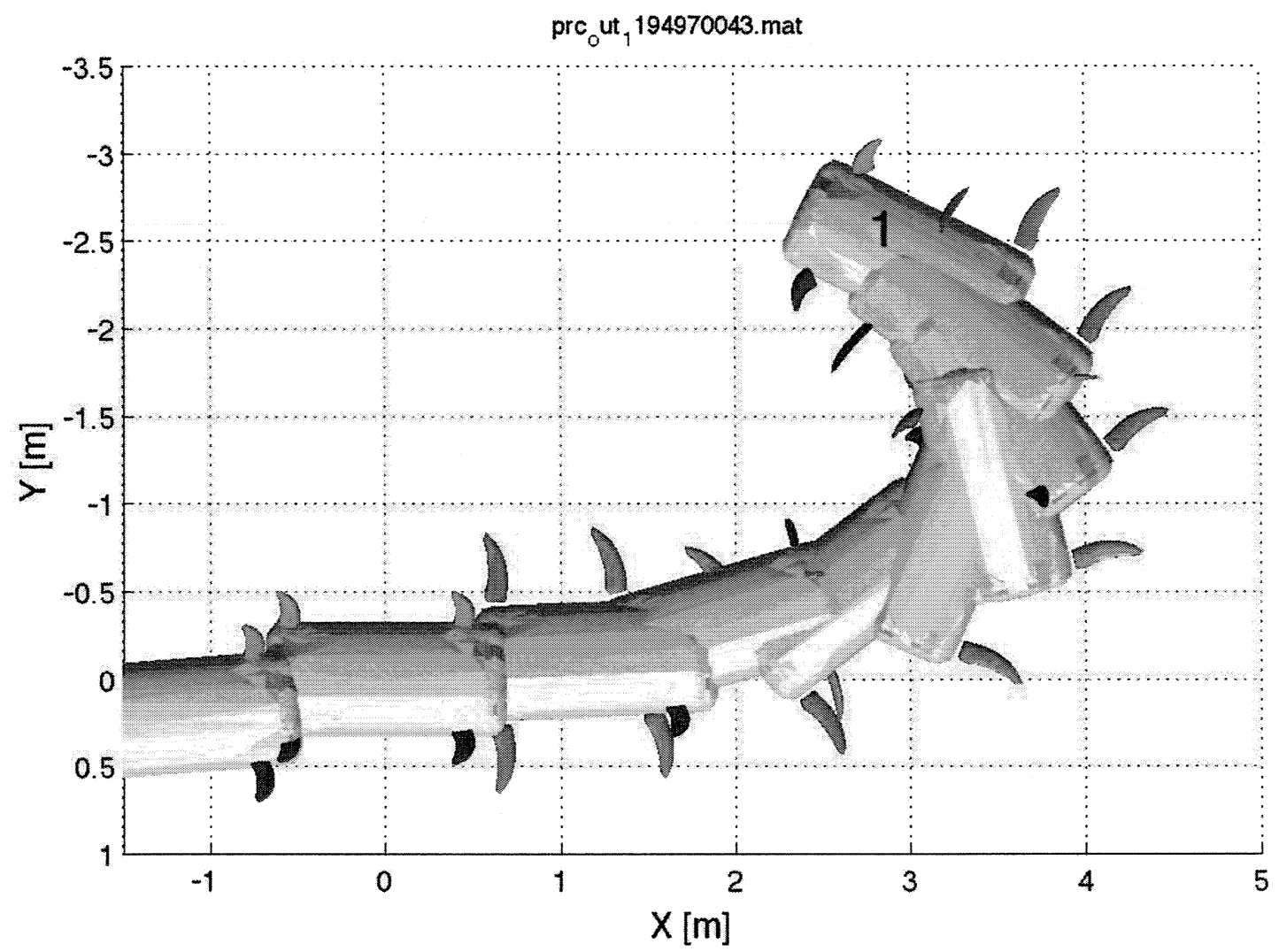

Figure 5-11: Top view of vehicle motion during representative biomimetic level turning trial, using both inside and outside forward foil for thrust. Case $\mathbf{B}$

as the vehicle exits steady forward swimming. While the lead up period to each maneuvering trial consists of the same number of steady swimming strokes, the vehicle is susceptible during this period to circulation patterns near the pool wall jets. In almost every case the vehicle enters the maneuver with slight positive roll and pitch angles $\left(<6^{\circ}\right)$.

The vehicle yaw rate closely tracks the foil motion, as shown in Figure 5-15 where yaw rate and yaw acceleration are plotted. The midpoint of each downstroke of the outside foil, marked with a solid vertical line in the figure, is immediately preceded by a peak in the vehicle yaw acceleration. The peak is followed by a smaller peak of the opposite magnitude, indicating deceleration in yaw during the second half of the outside foil downstroke. There is a another slight deceleration at the midpoint of 


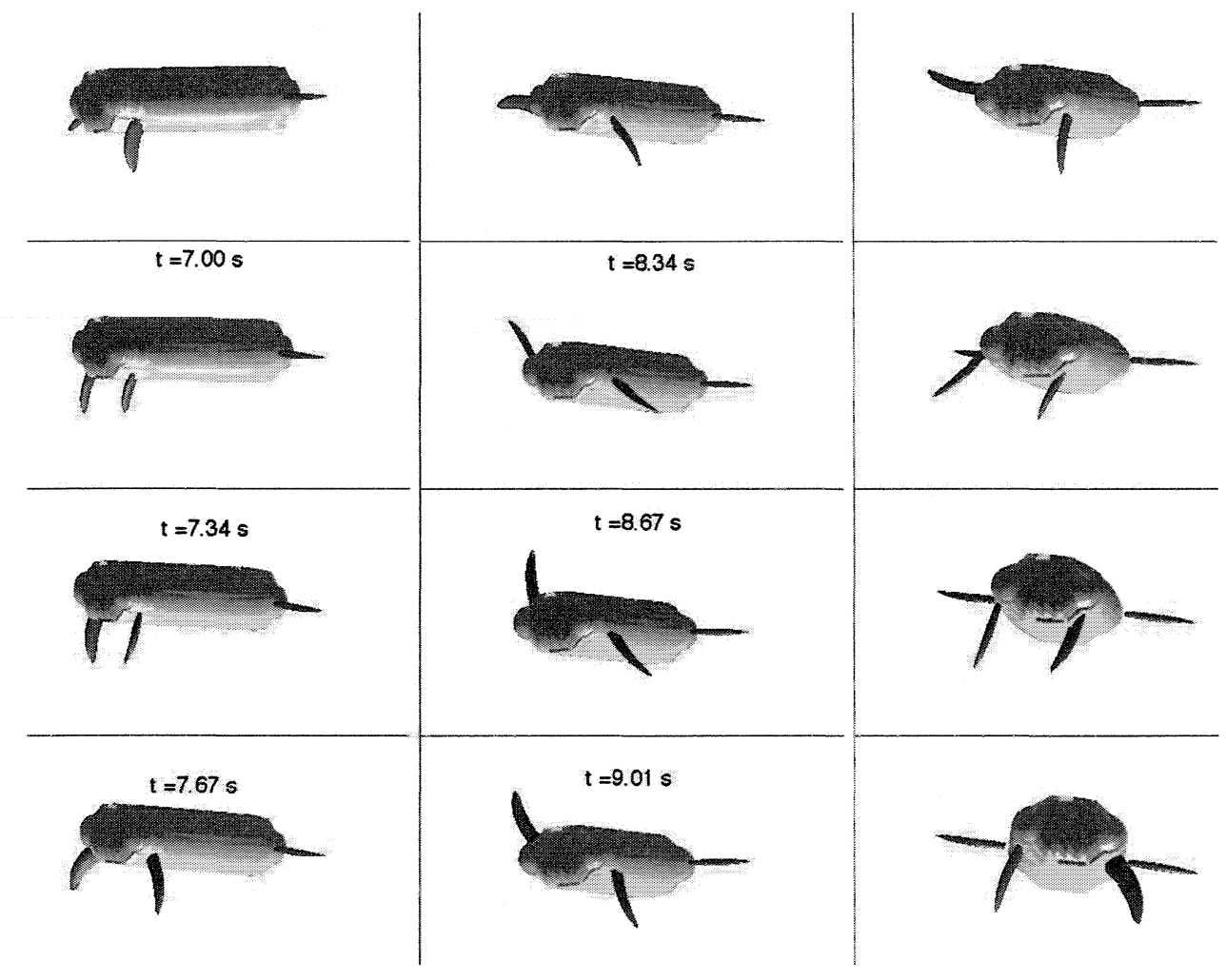

Figure 5-12: Cartoon for visualization of body and foil motion through a portion of a biomimetic level turn, with virtual camera angle and time interval designed to allow direct qualitative comparison to turtle behavior shown in Figure 5-13. Case $\mathbf{B}$

each outside foil upstroke, marked in the figure as dotted vertical lines. In Figure 514 the effect of the yaw acceleration that occurs with each downstroke is seen in corresponding knees in the yaw plot, representing almost discrete changes in yaw rate.

After $\mathrm{t}=2 \mathrm{~s}$, when the outside foil motion begins, the vehicle oscillates in roll with an amplitude of approximately $6^{\circ}$, generally in phase with the outside foil roll motion, but with a distorted positive peak occurring during the brief period when both foils are exerting positive roll moment (i.e. after the inside foil downstroke begins but before the outside foil upstroke has ended.) This distortion takes the form of inflection points in roll angle trace at $t=4.6 \mathrm{~s}$ and $\mathrm{t}=7.6 \mathrm{~s}$. At these points, the foil roll levels off but then begins to accelerate again at the start of the inside foil downstroke, driving the maximum roll higher before the outside foil downstroke reverses the roll direction. The pitch angle trends positive over the same time period, increasing by 


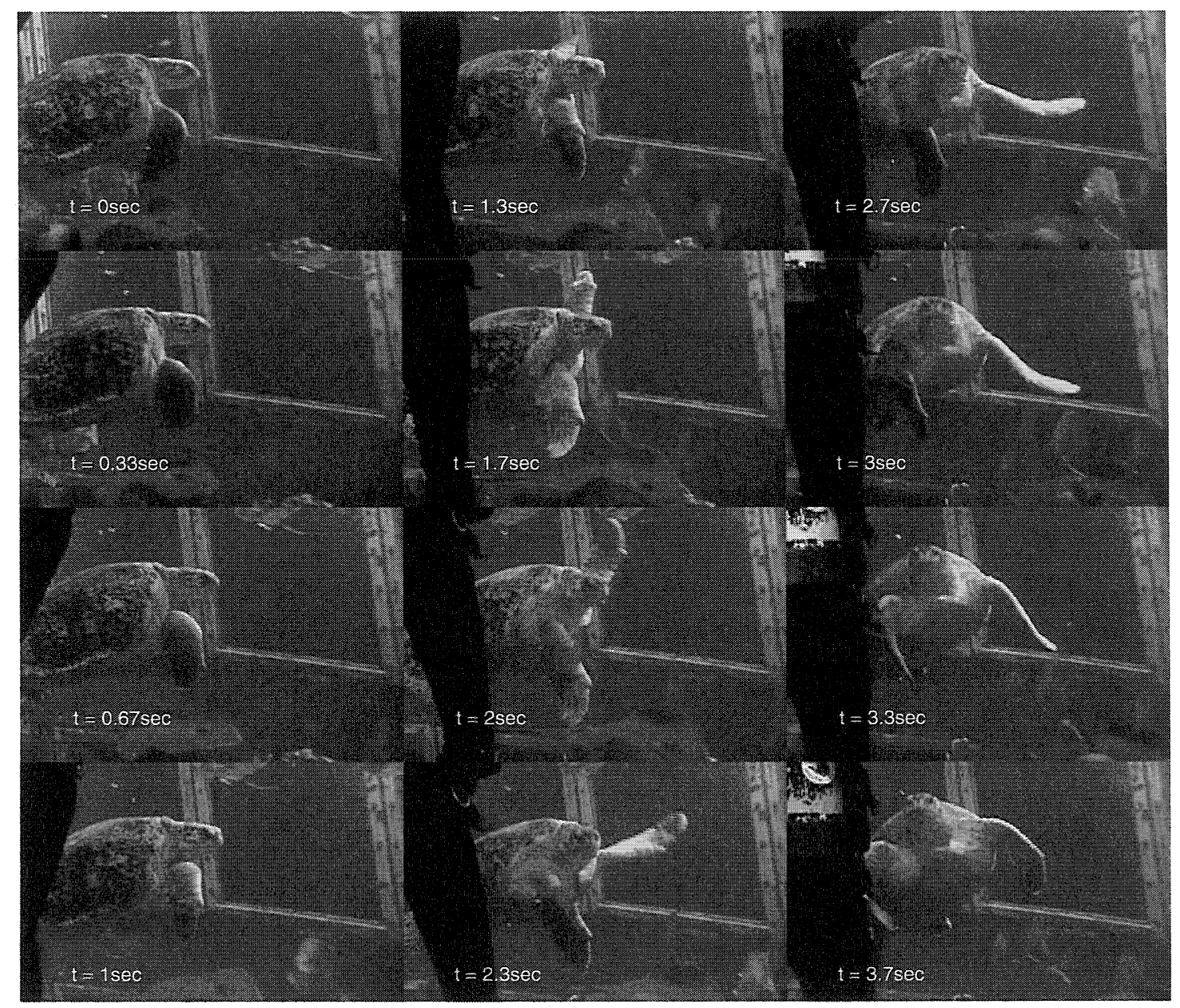

Figure 5-13: Screen captures from video of turtle performing a representative level turn with active participation of both forelimbs, repeated for clarity. This view highlights the extension of the outside forelimb as it is thrown forward just as the downstroke is initiated.

approximately $10^{\circ}$ from $\mathrm{t}=2$ to $\mathrm{t}=10 \mathrm{sec}$, as both foils are producing greater lift forces during the downstroke (where positive foil lift exerts a positive pitch moment on the body) than during the upstroke (when negative foil lifts exerts negative pitch moment.)

The results for cases $\mathbf{B}^{\prime}, \mathbf{B}^{\prime \prime}$, and $\mathbf{B}^{*}$ are qualitatively similar to those of case $\mathbf{B}$. The heading change and the maximum heading rate recorded over the course of each maneuver are compared to those of $\mathbf{B}$ in Table 5.1, given in terms of percent change.

Surprisingly, there is no significant increase in the heading change from Case $\mathbf{B}$, which utilizes a highly feathered recovery stroke, and Case $\mathbf{B}^{\prime}$, where the twist angle 


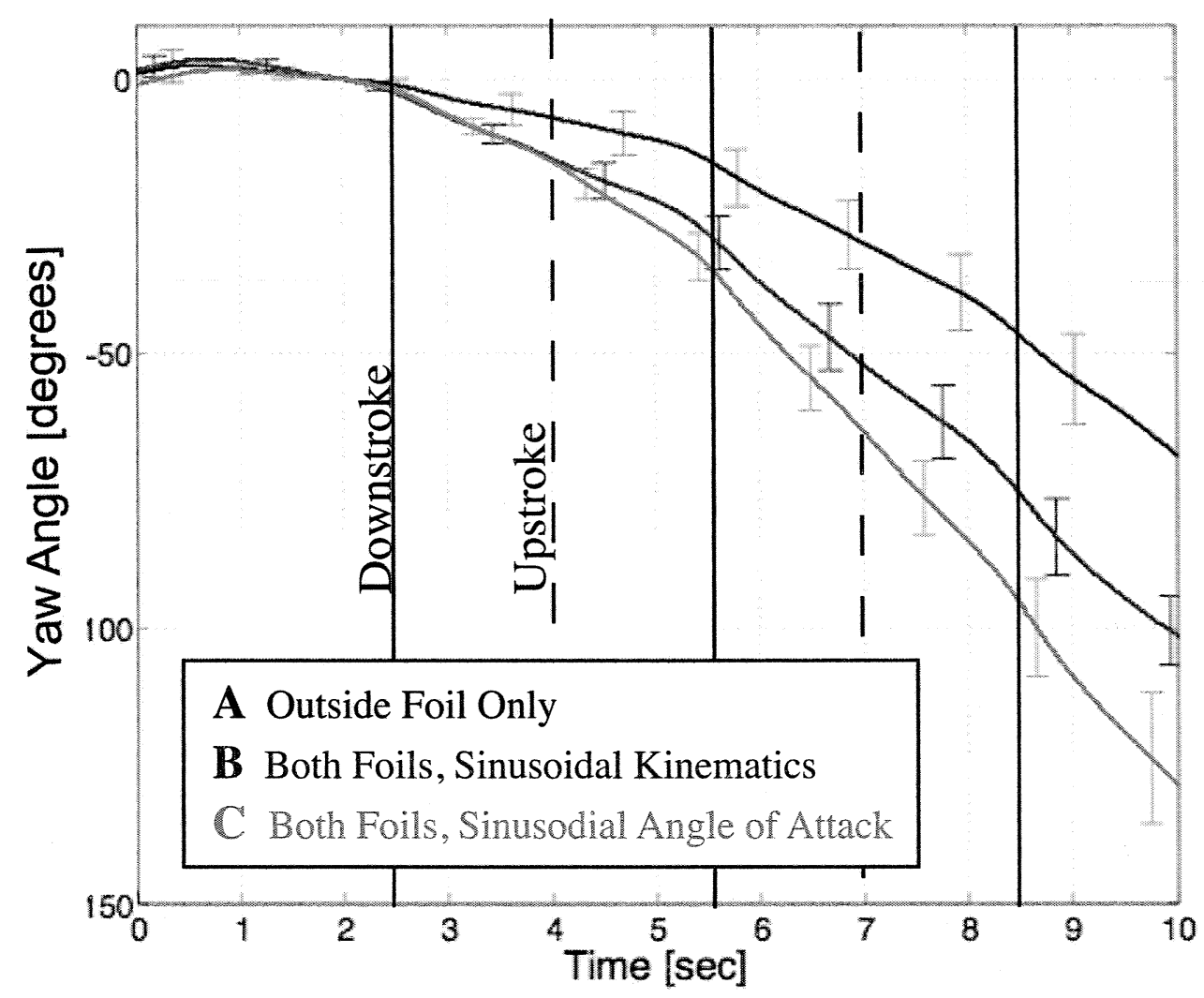

Figure 5-14: Heading and heading rate for three cases: with and without inside forelimb rowing motion, and with forelimb rowing motion using sinusoidal angle of attack profile instead of simple sinusoidal kinematics. Vertical lines indicate time at which foil crosses midpoint of roll for downstrokes and upstrokes.

on the upstroke is identical to the twist angle on the upstroke. The total range of the vehicle roll motion is increased, however, as the outside forelimb exerts a large roll moment on both the upstroke and the downstroke (see Figure 5-16 for roll angle through the turn for $\mathbf{B}, \mathbf{B}^{\prime}$ and $\mathbf{B}$ ".) The magnitude of the maximum roll excursion increases by $4^{\circ}(100 \%)$ and the minimum roll excursion by $3^{\circ}(50 \%)$

The roll excursion can be reduced for the symmetric case by increasing the twist amplitude to $45^{\circ}$ for both upstroke and downstroke, as in Case B", testing the possibility that the increased roll excursion hampers turning. However, while roll excursion is reduced for $\mathbf{B}$ " as shown in Figure 5-16, the effect is accompanied by a $15^{\circ}$ reduction in total heading change; if there is any direct benefit to thrust production in maintaining level, it is overwhelmed by the decrease in thrust expected when reducing the 

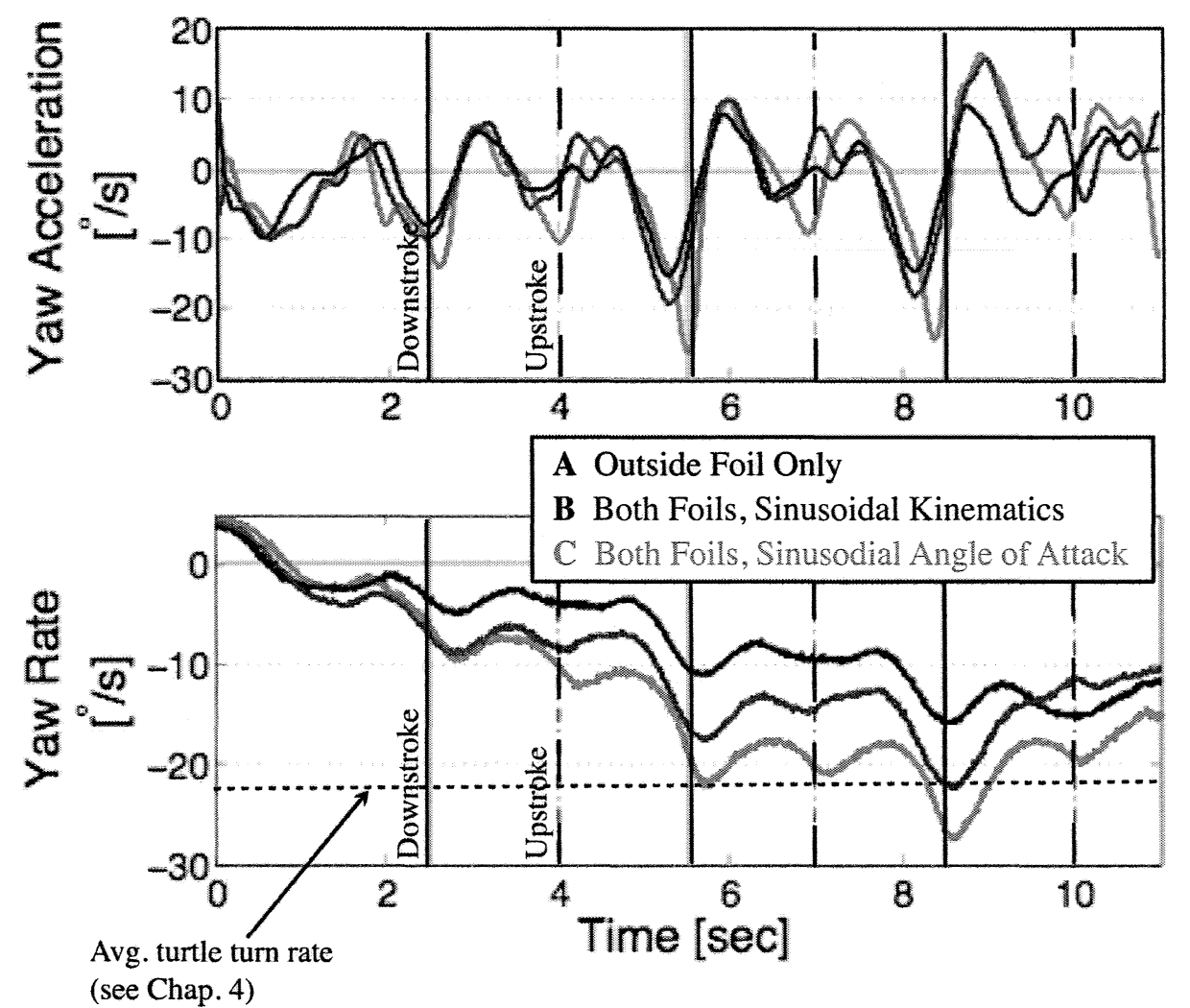

Figure 5-15: Heading and heading rate for three cases: with and without inside forelimb rowing motion, and with forelimb rowing motion using sinusoidal angle of attack profile instead of simple sinusoidal kinematics. Vertical lines indicate time at which foil crosses midpoint of roll for downstrokes and upstrokes.

maximum angle of attack on the foil from $30^{\circ}$ to $20^{\circ}$.

Even as roll excursion is increased, the vehicle pitch change over the course of the maneuver is reduced. As shown in Figure 5-17, the vehicle pitches up 50\% less, a possibly beneficial effect. During similar live animal maneuvers observed and recorded in Chapter 4, the turtle was pitched up significantly throughout; whether or not this is detrimental to the turtle, it could pose problems for a maneuvering vehicle attempting to remain level.

In $\mathbf{B}^{*}$ where the inside limb roll amplitude is decreased from $45^{\circ}$ to $35^{\circ}$ to allow for an increase in the roll bias from $75^{\circ}$ to $85^{\circ}$, the heading change achieved dropped slightly, by $2 \%$, as did the maximum heading rate, by $5 \%$. The small changes suggest 


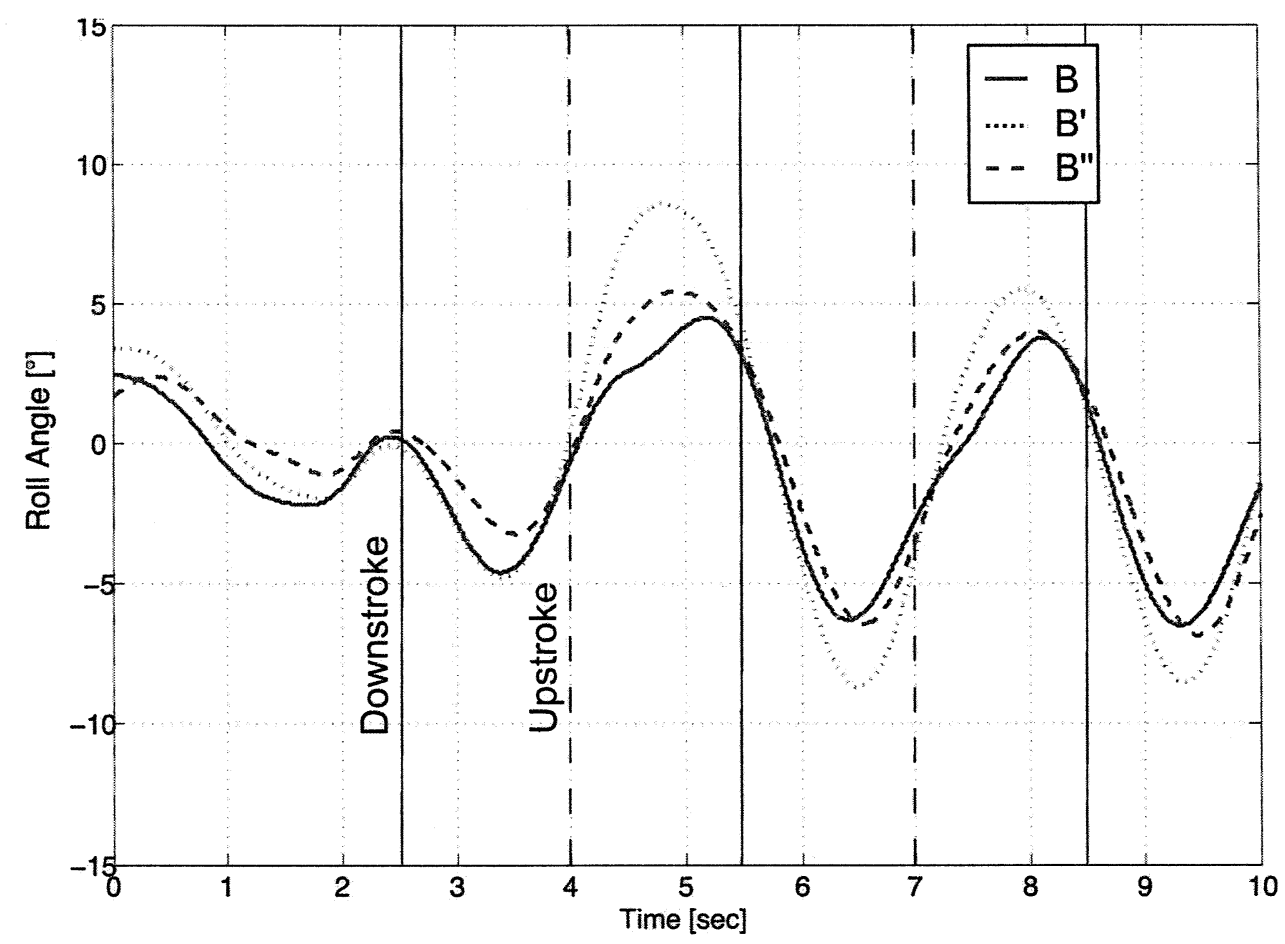

Figure 5-16: Body roll response to biomimetic level turning foil kinematics, for Cases B, B'. and B'. Attempting a power stroke on both the upstroke and the downstroke (B') leads to larger roll excursion with minimal turning performance difference than feathering the upstroke $(\mathbf{B})$. Using a power stroke for both, but increasing twist angle from $30^{\circ}$ to $40^{\circ}$ (B") does not increase roll excursion, but results in a drop in turning performance (see Table 5.1). Vertical lines indicate time at which foil crosses midpoint of roll for downstrokes and upstrokes.

low sensitivity to the kinematic parameters about the baseline kinematics.

\subsubsection{Lift Based (Outside) Foil Action Alone.}

The comparison between the turning performance with the inside foil, (B) and without the inside foil, (A), shows a significant reduction in both the total heading change and the maximum heading rate when the inside foil is stationary. The total heading change is reduced by $32 \%$, from $102^{\circ}$ to $68^{\circ}$, and the maximum heading rate by $27 \%$ from $22^{\circ} / \sec$ to $16^{\circ} / \mathrm{sec}$ (see Table 5.1). Heading and heading rate throughout the entire maneuver are compared to those of Case B in Figure 5-14 and Figure 5-15.

As with the combined foil kinematics, the yaw acceleration peaks just before the midpoint of the outside foil downstroke, but here, with just a single foil actuated, the 


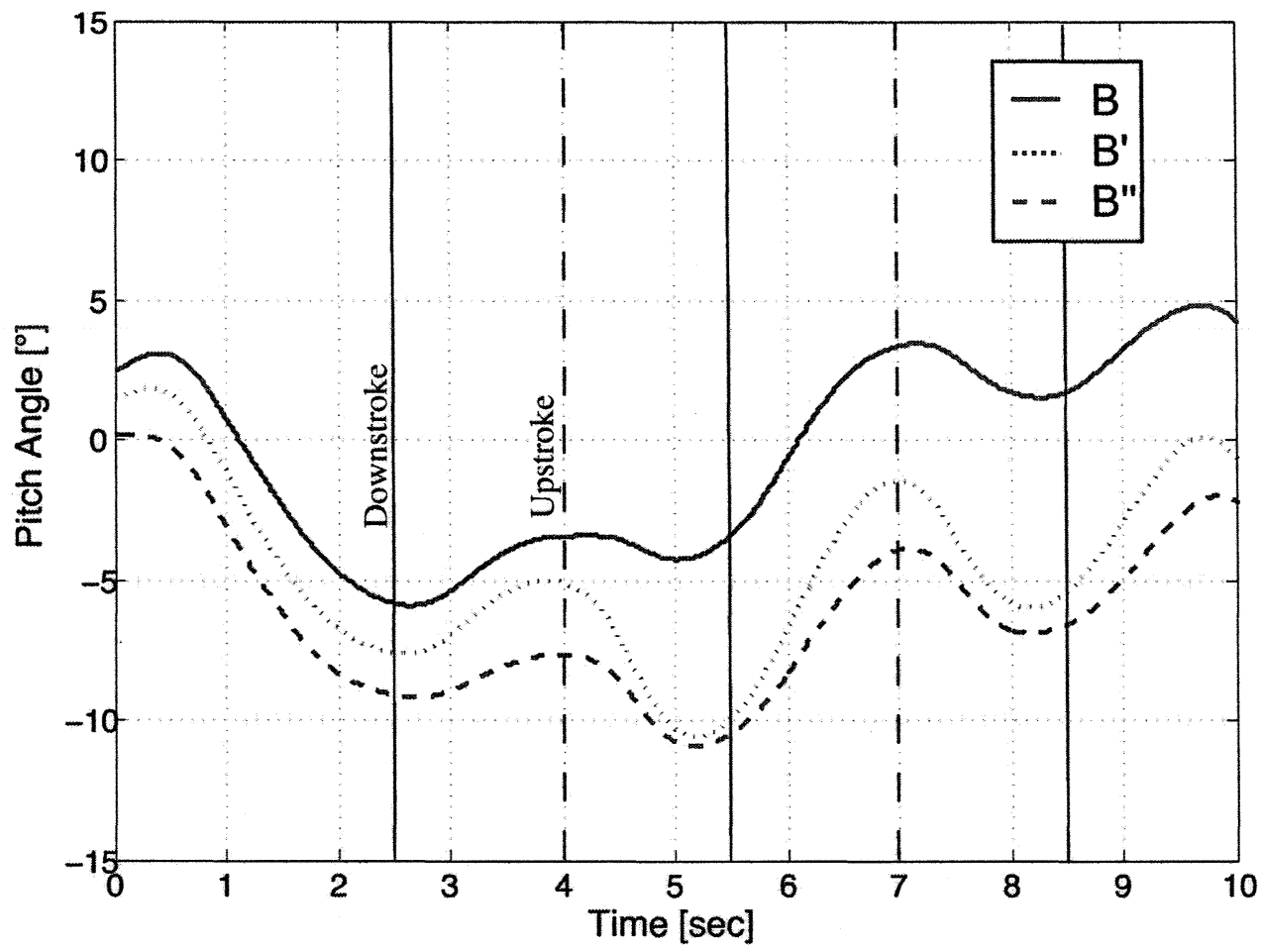

Figure 5-17: Body roll response to biomimetic level turning foil kinematics, for Cases B, B'. and B'. Attempting a power stroke on both the upstroke and the downstroke for the outside foil $\left(\mathbf{B}^{\prime}, \mathbf{B}^{\prime \prime}\right)$ reduces the tendency of the vehicle to pitch up during the level turning maneuver, in comparison to kinematics with a feathered upstroke (B). Vertical lines indicate time at which foil crosses midpoint of roll for downstrokes and upstrokes.

magnitude of the acceleration is smaller. In slight contrast, the small deceleration that occurs during the foil upstrokes in $\mathbf{B}$ is eliminated now that the inside foil recovery stroke is not required, but this does not compensate for the change in magnitude of the peak associated with the downstroke; the magnitude of the vehicle yaw rate is greater when both foils are actuated from $t=2$ seconds onward. The roll behavior also differs somewhat between the two cases. As might be expected, the small roll peak at $t=2.5$ s disappears with the removal of the inside limb downstroke preceding the first outside foil downstroke, and the distortion of the roll motion peak value around $t=5 \mathrm{~s}$ an $\mathrm{t}=8 \mathrm{~s}$ has been removed.

In Case $\mathbf{A}^{\prime}$ the kinematics of the outside foil were changed, as they were in Case $\mathbf{B}^{\prime}$, to use the same twist angle for the upstroke and the downstroke. Once again, this change has a surprisingly small effect on the final results, as shown in Table 5.1. 


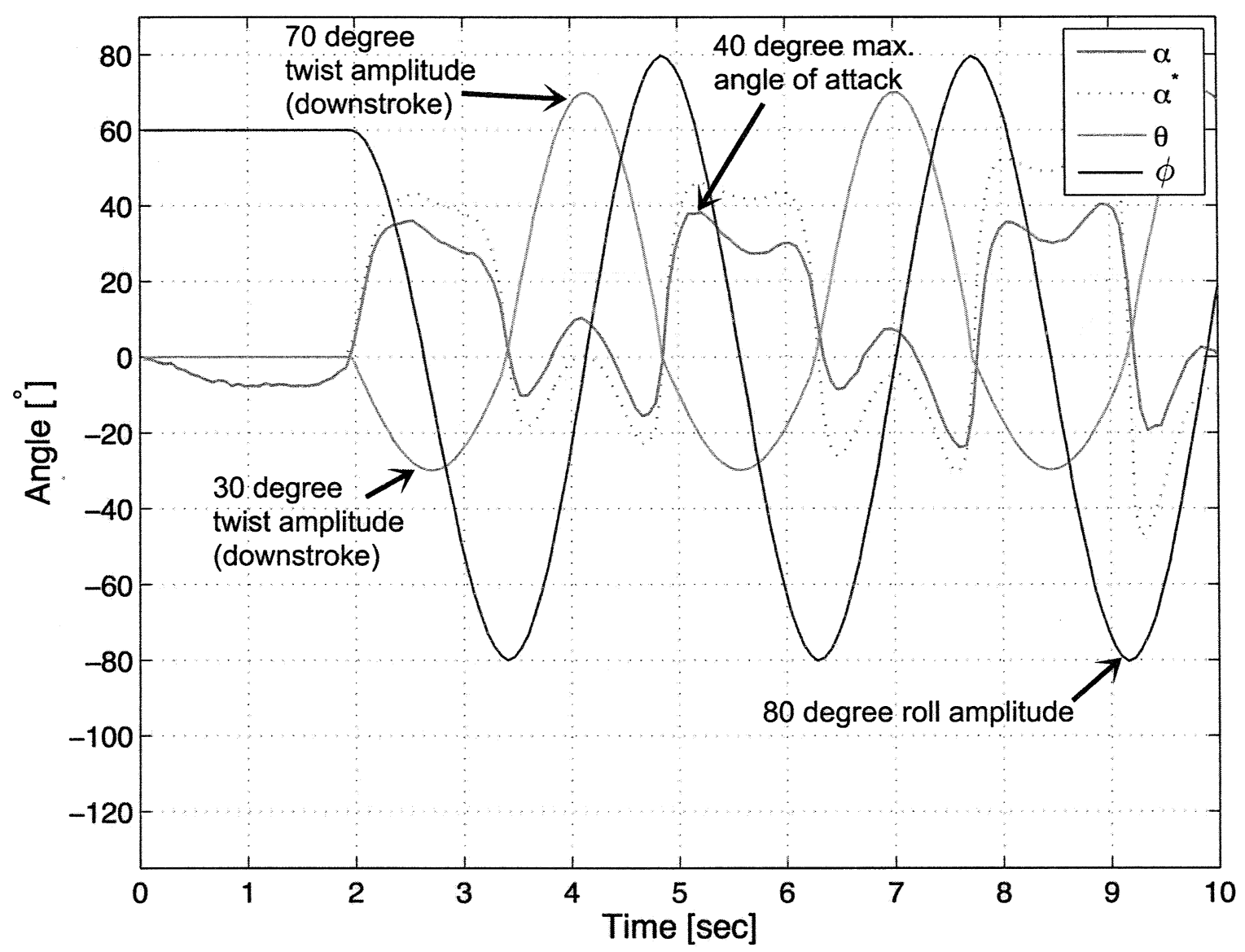

Figure 5-18: Sinusoidal foil kinematics and resulting nominal angle of attack at $70 \%$ span for outside forelimb during biomimetic level turn. The angle of attack profile that would obtain if the body had zero angular velocity $\left(\alpha^{*}\right)$ is indicated with a dotted line. Case $\mathbf{B}$.

\subsubsection{Combined Foil Action with Sinusoidal $\alpha(t)$.}

Figure 5-18 plots the outside foil angles for case $\mathbf{B}$ with the nominal angle of attack for the foil superimposed, calculated using the body motion recorded for that case. $\alpha$ is calculated at $r_{0.7}$ on the foil, as defined above. A degenerate angle of attack profile is clearly evident, with multiple maxima and minima for each stroke. Note that $\alpha_{\max }$, the maximum (positive) angle of attack for the downstroke, is much higher than the maximum (negative) angle of attack of the upstroke, $38^{\circ}$ vs. $10^{\circ}$ respectively, as a result of the greater twist amplitude on the upstroke.

Given the velocity of the vehicle at the beginning of the maneuver, and using the arc length at $70 \%$ span as the foil length scale, an effective Strouhal number of 


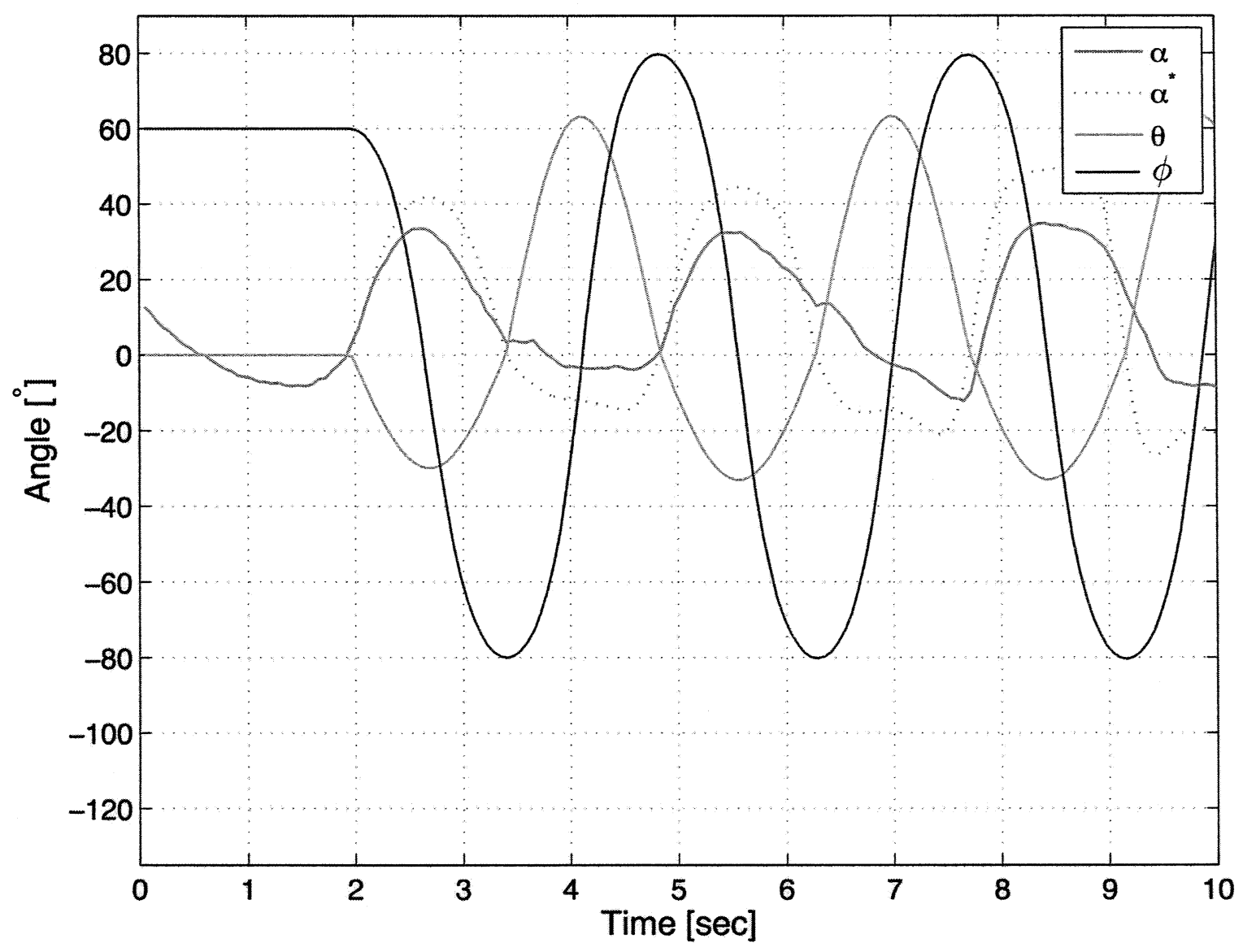

Figure 5-19: Foil kinematics calculated a priori to produce approximately sinusoidal nominal angle of attack profile, with $\alpha_{\max }=40^{\circ}$, at $70 \%$ span of outside forelimb during biomimetic level turn, with resulting nominal angle of attack from average vehicle response during experiments. The angle of attack profile that would obtain if the body had zero angular velocity is indicated as well. Case $\mathbf{C}$.

0.8 and $\alpha_{\max }^{D}$ of $35^{\circ}$ can be calculated for the first downstroke for Case B. With this relatively high Strouhal number and low $\alpha_{\max }$, it should be no surprise that a degenerate angle of attack profile obtains. (Note that the vehicle motion changes the shape of the angle of attack profile such that the two maxima during each downstroke are not identical, as they would in the idealized experimental case with steady foil translation. The same holds true for the minima during upstroke.) Through all three downstrokes strokes, the values of $\alpha_{\max }^{D}$ were $\left[35^{\circ}, 41^{\circ}, 38^{\circ}\right]$.

To allow for a direct comparison between Case $\mathbf{B}$, with sinusoidal $\theta$, and Case $\mathbf{C}$, with sinusoidal $\alpha$, the desired $\alpha_{\max }^{D}$ and $\alpha_{\max }^{U}$ for Case $\mathbf{C}$ were set to $40^{\circ}$ and $10^{\circ}$, respectively. A constant flow speed $U$ of $0.5 \mathrm{~m} / \mathrm{s}$ was assumed, lower than the 


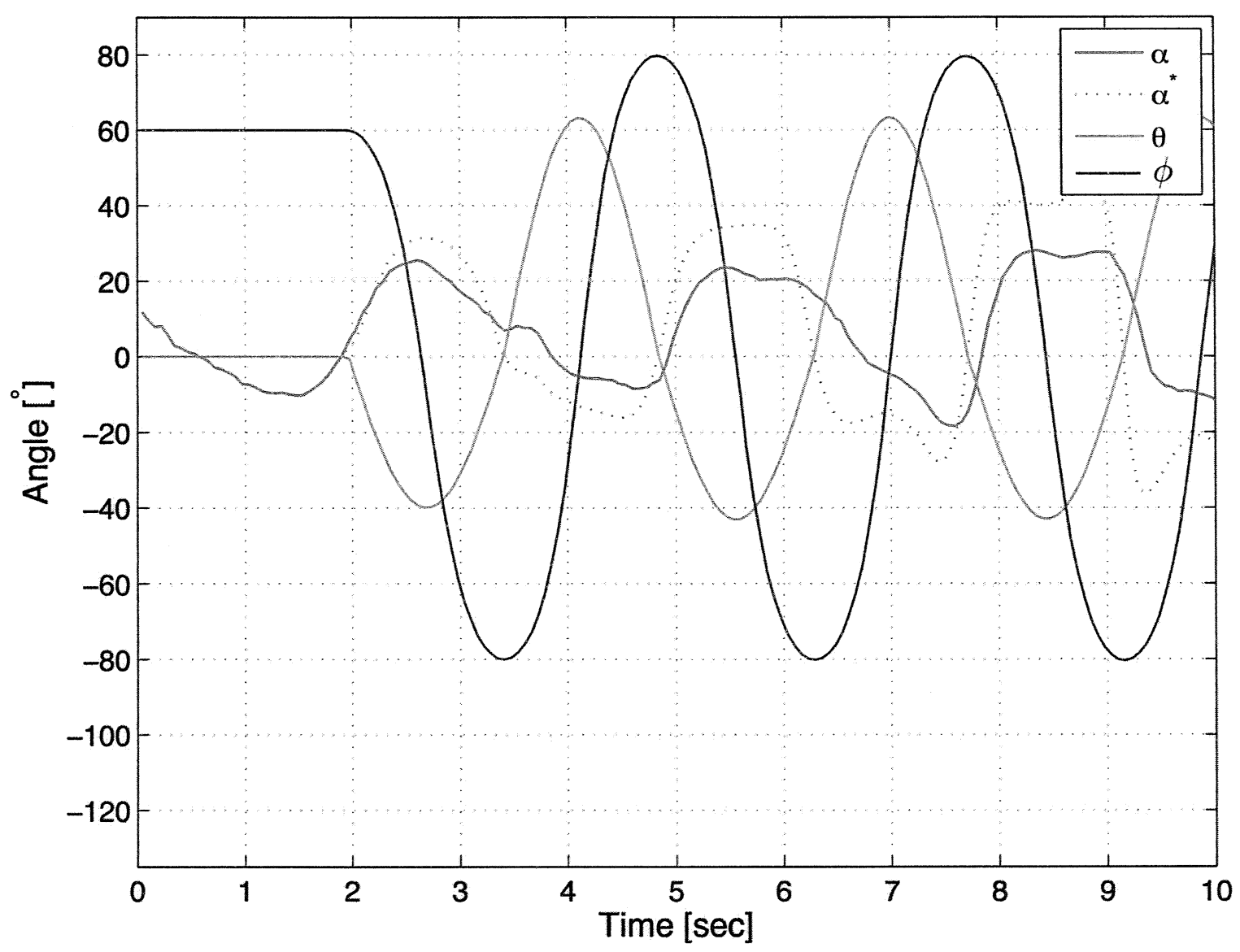

Figure 5-20: Foil kinematics calculated a priori to produce approximately sinusoidal nominal angle of attack profile, with $\alpha_{\max }=30^{\circ}$, at $70 \%$ span of outside forelimb during biomimetic level turn, with resulting nominal angle of attack from average vehicle response during experiments. The angle of attack profile that would obtain if the body had zero angular velocity $\left(\alpha^{*}\right)$ is indicated as well. Case $\mathbf{C}^{*}$.

nominal level cruising speed for $t=0$, but higher than the vehicle speed at $t=2.0$; the vehicle decelerates in all cases to $U=0.4 \mathrm{~m} / \mathrm{s}$ when forward thrust actuation halts during the transition period, as shown in plots of vehicle forward speed in Figure 527. Using the iterative procedure described in Section 5.2.1, downstroke and upstroke twist amplitudes were set to $\theta_{0}^{D}=28$ and $\theta_{0}^{U}=65$ and $\phi(t)$ calculated as shown in Figure 5-8.

The roll and twist angles of the outside foil are shown with the resulting angle of attack superimposed in Figures 5-19. The angle of attack that would have resulted if the vehicle were not rotating is also plotted, and can be seen peaking with the desired amplitude for the first downstroke. However, the angle of attack with vehicle rotation 


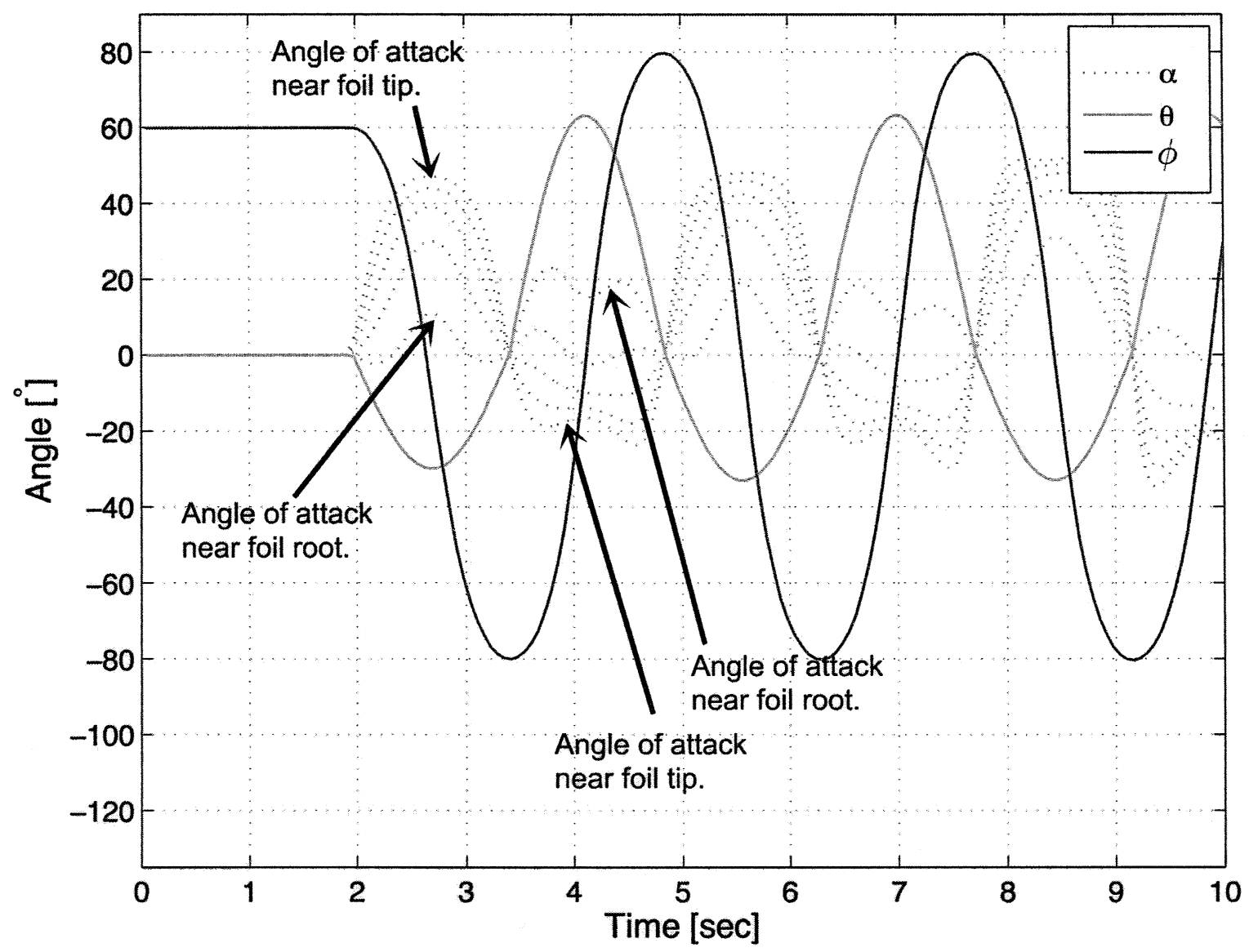

Figure 5-21: Illustration of the dependence of nominal angle of attack profile on spanwise location on foil. Kinematics used are calculated to produce $\alpha_{\max }=40^{\circ}$ at $70 \%$ span (Case $\mathbf{C}$ ). The dotted lines represent the calculated angles of attack occurring throughout the maneuver at five equally spaced points from root to tip of the foil. (To isolate effect of spanwise location, the effect of vehicle angular rates on angle of attack have been removed.)

accounted for has a lower peak; the maximum values for the three downstrokes are $\left[35^{\circ}, 34^{\circ}, 37^{\circ}\right]$. The shape of the profile is qualitatively very different; there is a single maximum for each downstroke, rather than two, and the peak occurs much close to the midpoint of the downstroke where the foil is moving with its maximum angular velocity. The shape of $\alpha(t)$ during the upstroke is also dramatically different from the simple sinusoidal kinematics. Where the simple sinusoidal kinematics produce a sharply double peaked profile, with the angle of attack crossing zero twice, the repaired kinematics keep the angle of attack negative throughout.

The effect on the vehicle turning motion is clear from the yaw acceleration and 
yaw rate as plotted in Figure 5-15. The yaw acceleration peaks corresponding to each downstroke are delayed relative to $\mathbf{A}$ and $\mathbf{B}$, just as the peaks in $\alpha$ are, and they are of significantly larger magnitude (although not as broad.) The magnitude of the resulting yaw rate is greater than both $\mathbf{A}$ and $\mathbf{B}$ for all $t>2$ seconds. From Figure 5-14 and Table 5.1, there is a $27 \%$ improvement in heading change and a 25\%f improvement in maximum heading rate over the simple sinusoidal kinematics.

Where downstroke $\alpha_{\max }=30^{\circ}$ (Case $\mathbf{C}^{*}$, shown in Figures 5-19,) the actual maximum values are $\left[26^{\circ}, 23^{\circ}, 28^{\circ}\right]$, significantly lower than the values for the degenerate case. Here, the shape of the profile is more sensitive to the reduction in nominal flow speed encountered by the foil through the second and third strokes. While the profile has a single peak near the midpoint of the first downstroke, the second and third downstrokes exhibit degenerate dual peak profiles, resulting from the failure to recalculate foil kinematics with new velocity information as the vehicle forward speed decelerates throughout the maneuver. Despite this, the overall result from these kinematics still show significant improvements over the simple sinusoidal case, with a $20 \%$ improvement in heading change and a $14 \%$ improvement in maximum heading rate (see Table 5.1.)

As a reminder that the nominal angle of attack traces shown in previous figures are specific to a location $70 \%$ of the distance along the span from root to tip, Figure 521 shows the nominal angle of attack at distances of $[0.1 \mathrm{~m}, 0.2 \mathrm{~m}, 0.3 \mathrm{~m}, 0.4 \mathrm{~m}]$ along the $0.4 \mathrm{~m}$ length of the foil. The instantaneous linear velocity of a point on the foil increases in proportion to the distance from the rotation axis in roll, which has the effect of increasing the nominal angle of attack of the foil closer to the tip. Analysis based solely on the angle of attack at a particular point along the foil does not account for spanwise flow along the foil, and cannot account for the inherently three dimensional structure of the foil wake. 


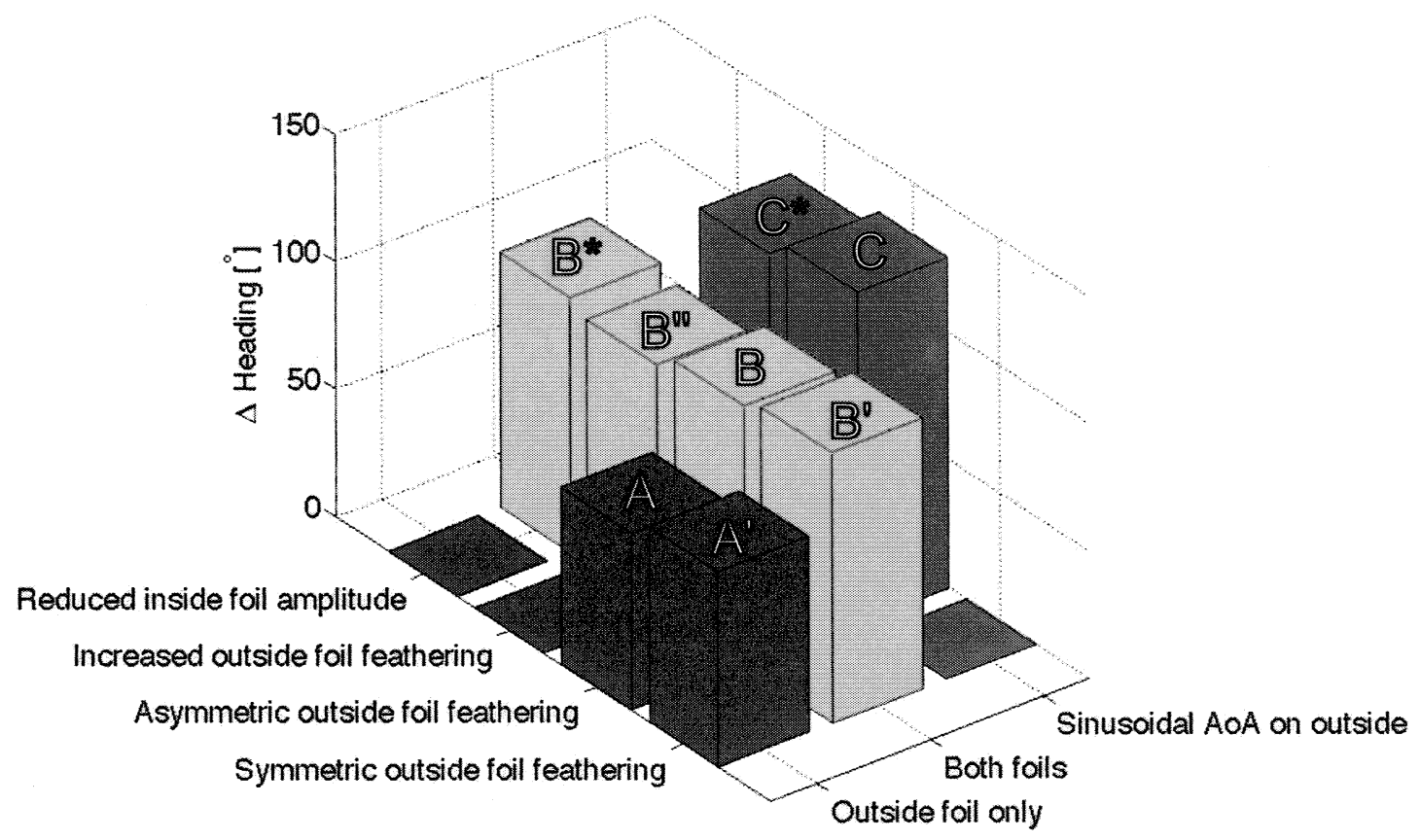

Figure 5-22: Comparison of heading change after completion of maneuver for variations on the biomimetic level turning kinematics, as given in Table 5.1

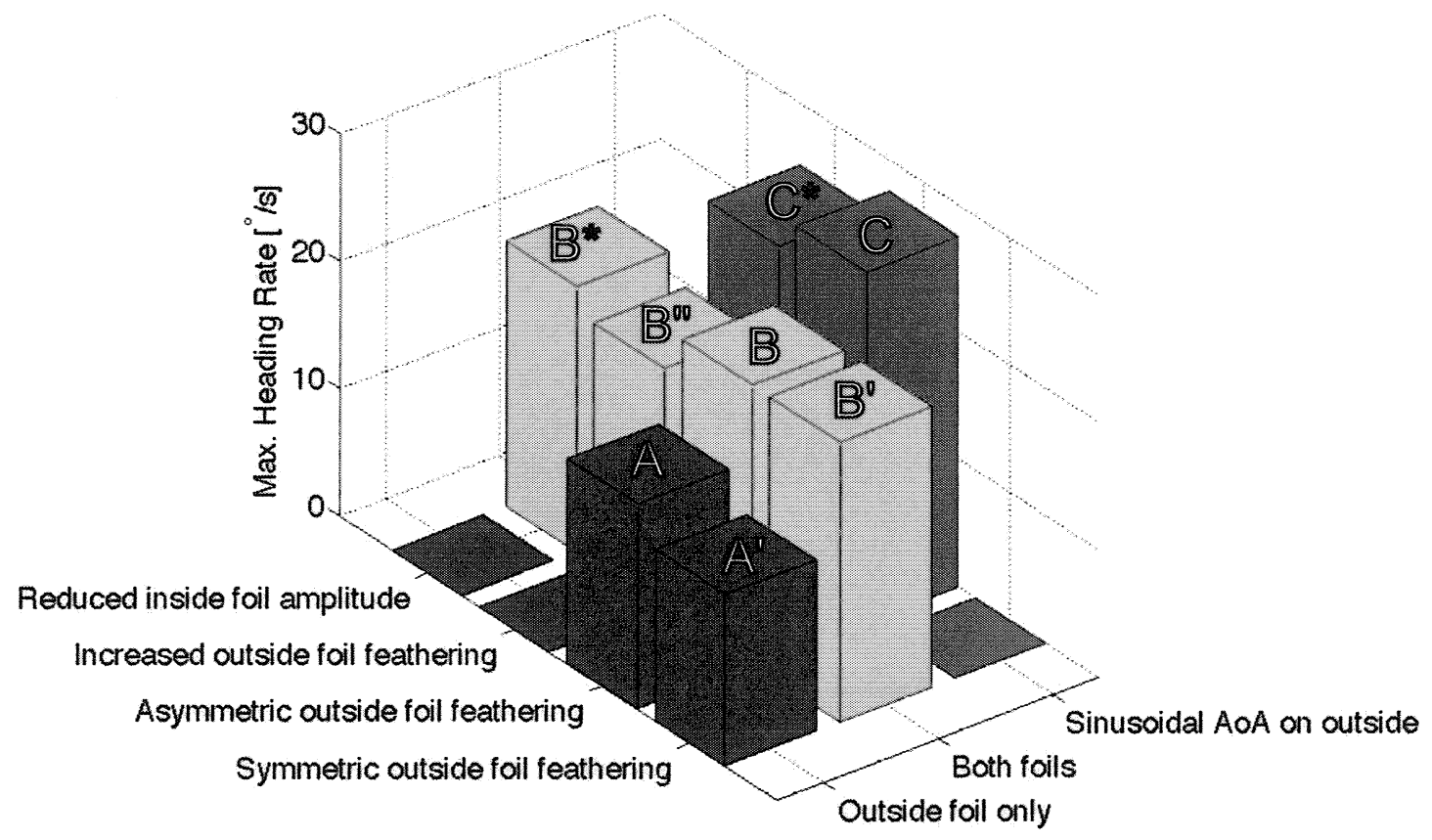

Figure 5-23: Comparison of maximum heading rate during maneuver for variations on the biomimetic level turning kinematics, as given in Table 5.1 
Kinematics

Results

\begin{tabular}{|c|c|c|c|c|c|c|c|c|c|}
\hline & $\phi^{I}$ & $\theta_{D}^{O}$ & $\theta_{U}^{O}$ & $\alpha_{D}^{O}$ & $\alpha_{D}^{O}$ & $\delta \Phi\left[^{\circ}\right]$ & $+\%$ & $\dot{\Phi}_{\max }\left[\frac{\circ}{\mathrm{sec}}\right]$ & $+\%$ \\
\hline \multicolumn{10}{|c|}{ Outside foil only, with sinusoidal $\theta(t)$} \\
\hline $\mathbf{A}$ & $\mathbf{0}$ & & & - & - & & -32 & & -27 \\
\hline$\overline{\mathbf{A}^{\prime}}$ & $\mathbf{0}$ & & 30 & - & - & & -25 & & -39 \\
\hline
\end{tabular}

Both foils, with sinusoidal $\theta(t)$ on outside foil

\begin{tabular}{|c||c|c|c|c|c||rc|c|}
\hline $\mathbf{B}$ & 45 & 30 & 70 & - & - & $102 \pm 5$ & $22 \pm$ \\
\hline $\mathbf{B}^{\prime}$ & & & $\mathbf{3 0}$ & - & - & +4 & 0 \\
\hline $\mathbf{B}^{*}$ & & $\mathbf{4 0}$ & $\mathbf{4 0}$ & - & - & -6 & -14 \\
\hline $\mathbf{B}^{* *}$ & $\mathbf{3 0}$ & & $\mathbf{3 0}$ & - & - & -2 & -5 \\
\hline
\end{tabular}

Both foils, with sinusoidal $\alpha(t)$ on outside foil

\begin{tabular}{|c||c|c|c|c|c||r|r|}
\hline $\mathbf{C}$ & & - & - & $\mathbf{4 0}$ & $\mathbf{0}$ & +27 & +25 \\
\hline $\mathbf{C}^{*}$ & & - & - & $\mathbf{3 0}$ & $\mathbf{0}$ & +20 & +14 \\
\hline
\end{tabular}

Table 5.1: Summary of Results: Biomimetic Level Turning Maneuvers

\subsection{Discussion}

The results from all of the adaptations of the biomimetic level turning kinematics are presented in Table 5.1 with graphical comparisons in Figure 5-22 and Figure 5-23. In Cases $\mathbf{A}$ and $\mathbf{A}^{\prime}$ a single foil (the foil to the outside of the turn) is used with sinusoidal roll and twist in a lift based stroke; in the baseline Case $\mathbf{B}$, along with variations B', B", and B* both foils are used with sinusoidal roll and twist, with the inside foil performing a drag base swimming stroke; and in Cases $\mathbf{C}$ and $\mathbf{C}^{*}$ both foils are used, with kinematics of the outside foil stroke modified to produce an approximately sinusoidal angle of attack.

From inspection of Figure 5-22 and Figure 5-23, the sensitivity of the overall turning performance to the tested parameter variations is low within all three of the major adaptations, when compared to the much larger difference in performance across the three adaptations.

For Case A, using only the foil to the outside of the turn, the best heading change achieved over a three stroke maneuver was $68^{\circ}$. The path taken by the vehicle during one experiment is shown in Figure 5-24. In Case $\mathbf{B}$, after the inside foils is recruited in a drag based stroke, made possible by the biologically inspired increase in actuator roll range, the heading change increases to $102^{\circ}$, and the maximum 


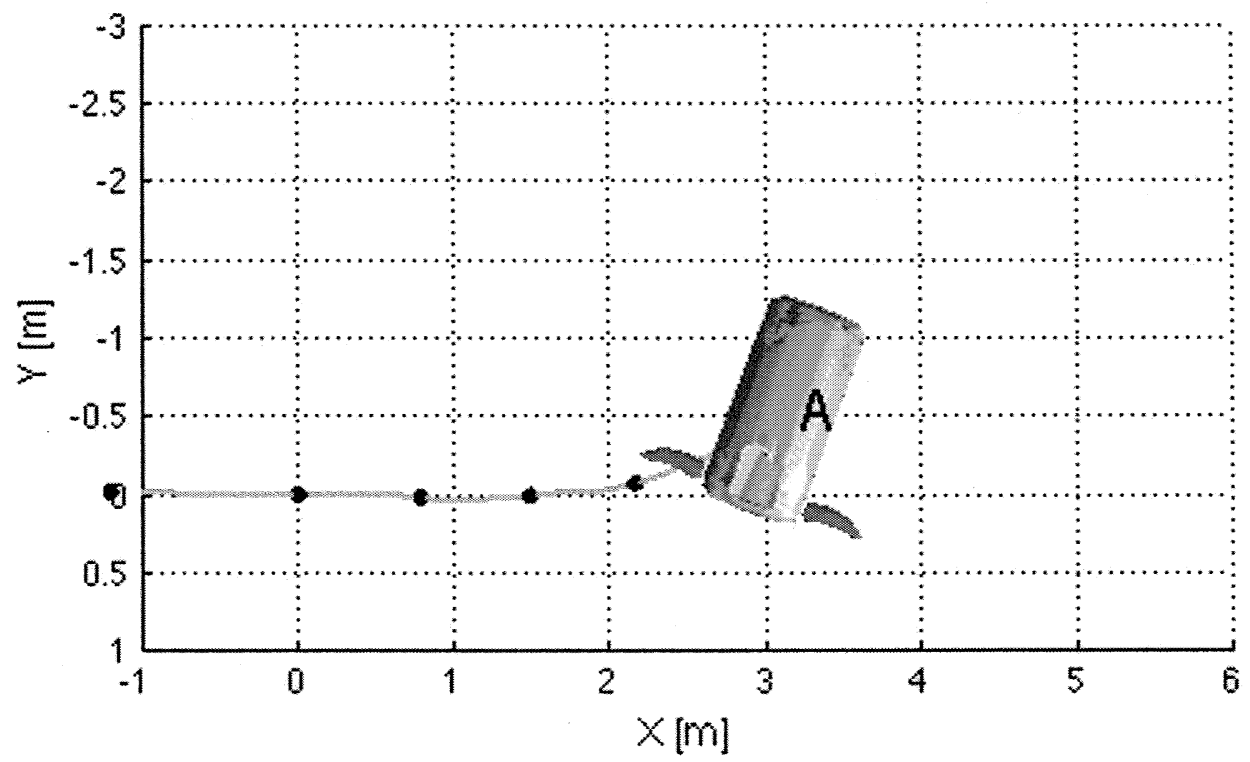

Figure 5-24: Top view, vehicle track and vehicle orientation at $t=10$ seconds for $a$ trial where only the outside foil is used to actuate the turn. (Case A)

heading rate increases from $16^{\circ} / \mathrm{s}$ to $22^{\circ} / \mathrm{s}$. With the simple sinusoidal kinematics, the outside foil is responsible for most of the turning moment, but the the inside foils significantly improves turning performance. This result matches the observation that the outside forelimb was dominant during level turning for Myrtle, as she was able to turn either with both forelimbs, or using just the outside forelimb. The vehicle path for representative trial of Case $\mathbf{B}$ is shown in Figure 5-25.

The most significant result, with implications for maneuvering and swimming behaviors beyond the level turning case examined in this chapter, is the $25 \%$ improvement in turning performance produced by changing from simple sinusoidal kinematics, B, to kinematics which produce a nominally sinusoidal angle of attack profile, $\mathbf{C}$. The 


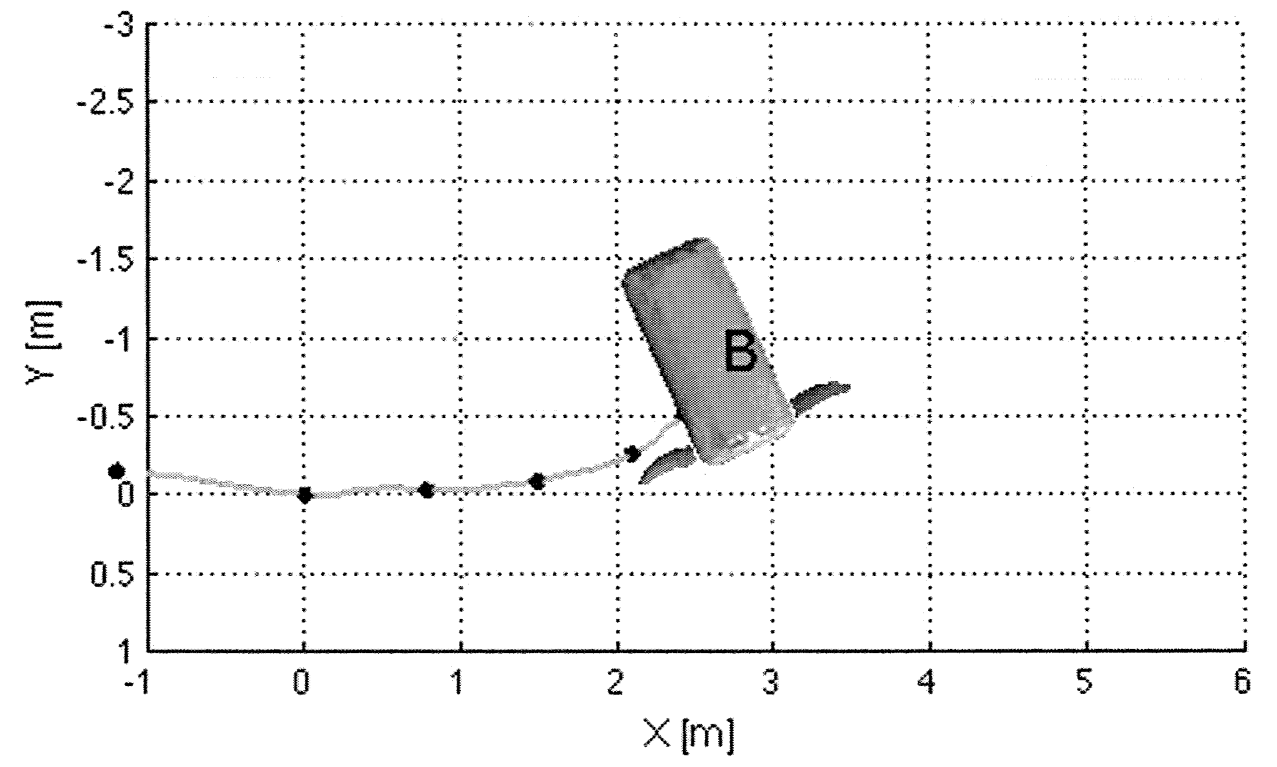

Figure 5-25: Top view, vehicle track and vehicle orientation at $t=10$ seconds for a trial where both foils are used to actuate the turn, with sinusoidal twist angle on the outside foil. (Case B)

difference can be seen by comparing the vehicle orientation after 10 s in Figures 5-25 and 5-26. Compare the turning rates for the three sets of kinematics, plotted in Figure $5-14$, to the average turning rate of $21-24^{\circ} / s$ observed during the second stroke of the turtle turn depicted in Figure 4-7. The turning rates for all three are increasing through the first two strokes, with the two fastest turns leveling out by the third stroke; the use of sinusoidal attack kinematics brings the average turning rate in line with the estimated average turning rate of the turtle. The present results indicate not only that tailoring the kinematics to produce a specific nominal angle of attack profile at a single point along the foil span can be effective in improving performance, but that this effect is robust to major simplifying assumptions when estimating the in- 


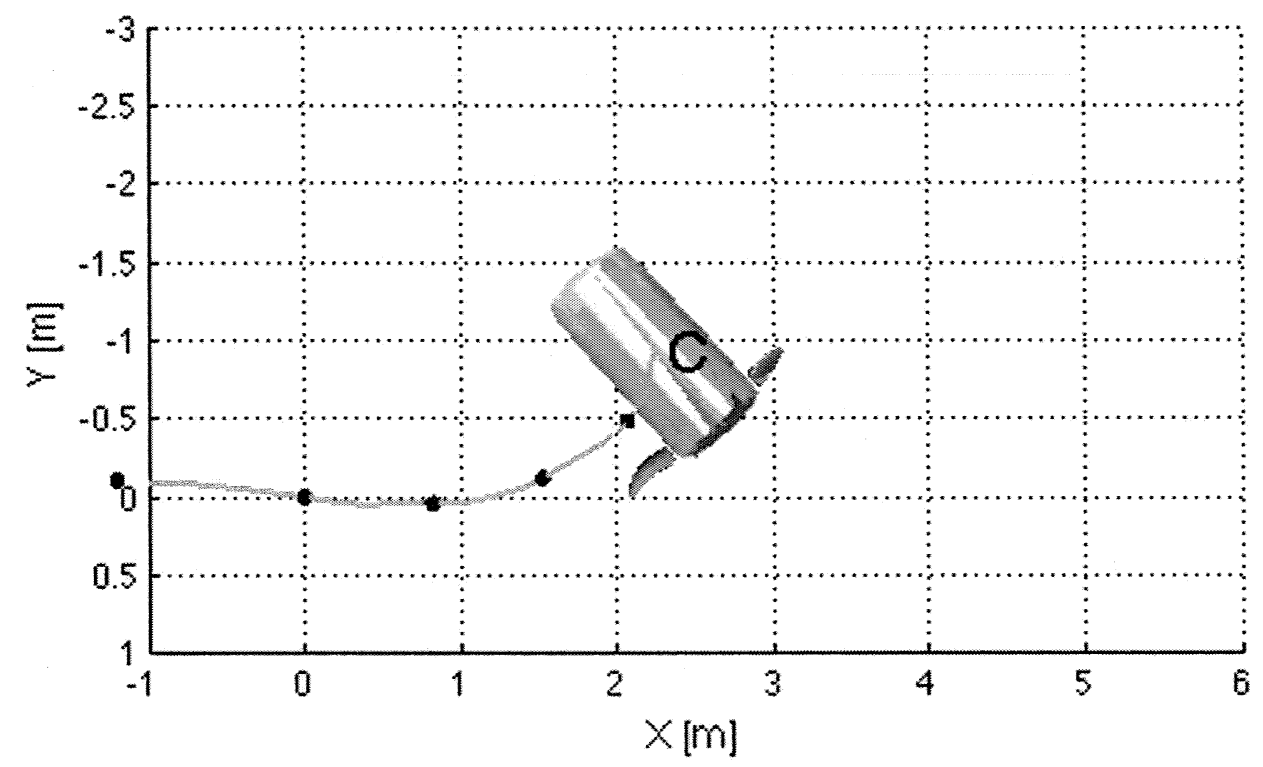

Figure 5-26: Top view, vehicle track and vehicle orientation at $\mathrm{t}=10$ seconds for a trial where both foils are used to actuate the turn, with sinusoidal angle of attack on the outside foil. (Case $\mathbf{C}$ )

coming flow. [27] and [55] both report that both efficiency and thrust increase across all values of Strouhal number and maximum angle of attack $\alpha_{\max }$ in two dimensional (heaving and pitching) foil motion. To our knowledge, however, no previous experiments have been carried out verifying that this holds for the more complex case three dimensional case, whether for a single foil or for a foil on a free-swimming vehicle.

Finally, in all cases the surge velocity of the vehicle steadily declined, and sway velocity steadily increased (see Figure 5-27), indicating that the vehicle was sideslipping as it followed the tracklines in Figure 5-28. (The sharp decline for $t<2$ seconds occurs during the nearly unactuated transition phase.) As shown in Chapter 4, a rigi 

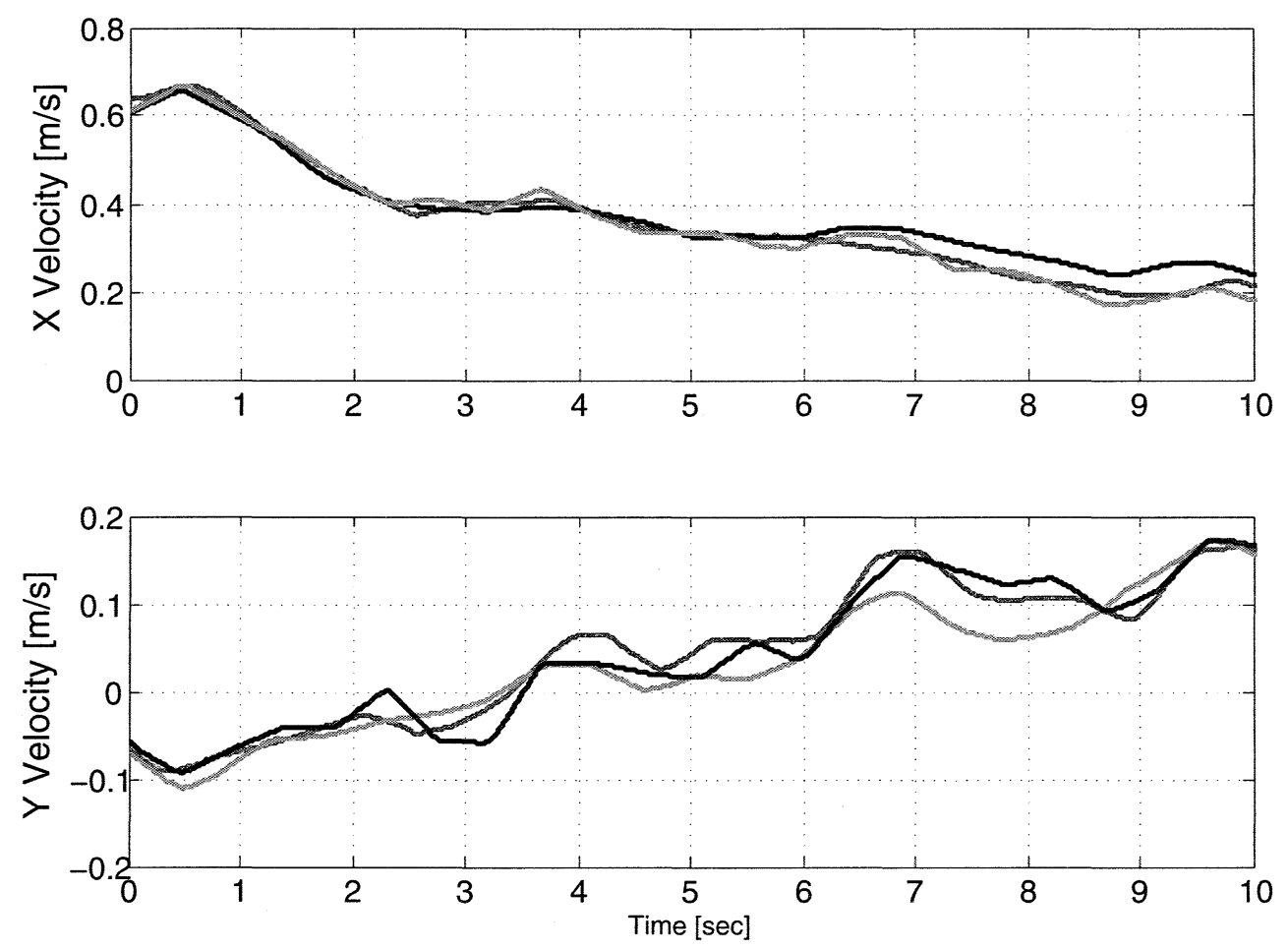

Figure 5-27: Body fixed vehicle surge and sway velocity for the baseline case for the three adaptations of the biomimetic level turn, Case A, B, and $\mathbf{C}$ (see Table 5.1 for comparson of foil kinematics.) 


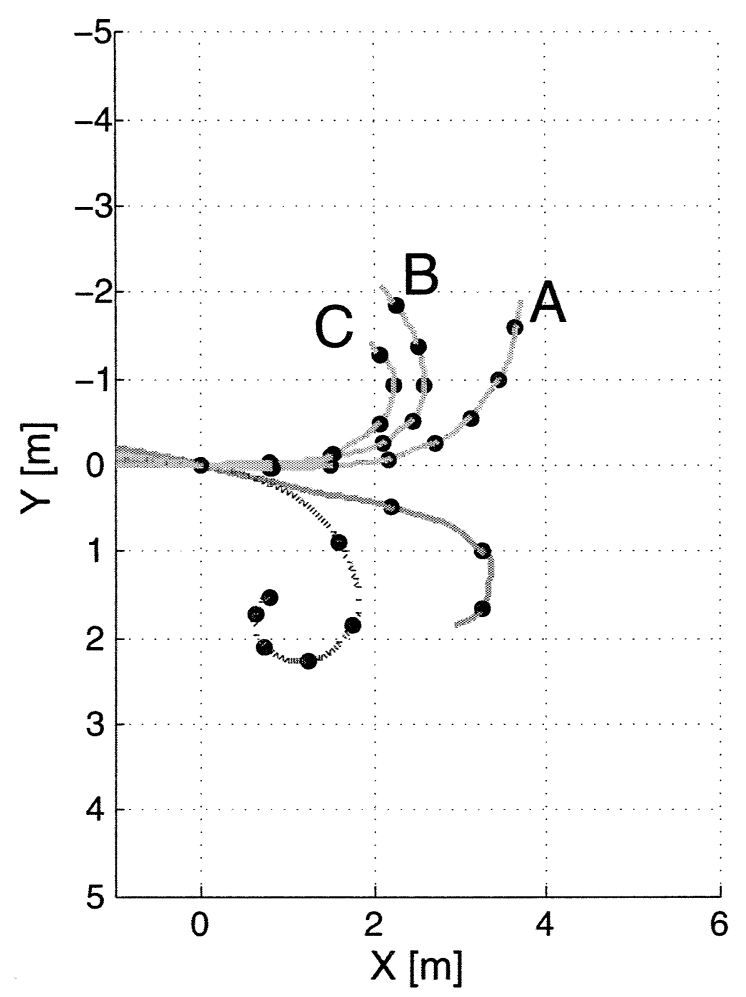

Figure 5-28: Top view, comparison of turning performance between turns using harmonically oscillating foils and biomimetic turn with both limbs participating, and a sinusoidal angle of attack profile on the outside limb. 


\subsection{Conclusion}

Despite significant morphological differences between turtle and vehicle, adaptations of the turtle limb kinematics were successfully used to execute turning maneuvers at low speeds, with a turning radius under $1 \mathrm{BL} / \mathrm{s}$ achieved. Finnegan was designed with two identical pairs of 2-DOF oscillating foils in order to simplify the control challenges of independent 6-DOF control in hovering and cruising tasks, while sea turtles use two high aspect ratio foils forward, and a pair low aspect ratio foils aft, both with more degrees of freedom and greater range of motion than the vehicle actuators. A biologically inspired redesign of the foil actuators, sacrificing actuator symmetry to mimic the increased downward range of a green turtle, significantly improved the success with which Finnegan executed heading changes.

Matching the gross parameters of the turtle limb motions while adapting the kinematics to produce a favorable angle of attack profile further improved vehicle turning performance, showing that fundamental understanding of thrust mechanism can be applied on a free swimming vehicle despite limited flow information. The results lead to the intriguing question of the whether the limb kinematics of sea turtles, and indeed of other high aspect ratio pectoral fin swimmers such as diving birds, are such that some approximation of a sinusoidal angle of attack obtains. Active control of chord-wise conformability could allow a sinusoidal angle of attack over a significant portion of the foil span, although spanwise effects presumably play a large role and could easily be the dominant factor in determining optimal chord shape as a function of spanwise position. This is research thrust well worth pursuing, and one which may be significantly advanced with the use of foil mounted flow or pressure sensing arrays, such as proposed in Fernandez [11].

When using a biomimetic approach to design, regardless of the system in question, it is important to keep an open mind to the possibility of replicating biological design and behavior at different levels of abstraction. In the previous chapters, the biological inspiration was more general, in that the concept of oscillating foils was distilled into a simplified form in the vehicle actuators, and an engineering approach was taken to 
determine effective control approach. In this chapter, the biological inspiration was direct and specific, with experiments mimicking a single organism's behavior in order to achieve the same goal as that of the organism, namely to change direction rapidly. In both cases, the biomimetic approach proved to be a effective design tool. 


\section{Chapter 6}

\section{Bioinspired Inline Motion in 2-D Oscillating Foils}

\subsection{Introduction}

The thrust production of oscillating foils has been extensively studied under laboratory conditions, but these experiments have generally focused on periodic motions with the foil free to move only transversely to a steady incoming flow e.g. [27], [53]. The number of kinematic parameters is reduced when this restriction is imposed, but the restriction does not accurately reflect biological examples of high-aspect ratio flapping propulsion, as shown in observational studies of steady swimming in birds [46], reptiles [33], mammals[67], and fish [40].

Sea turtles, by demonstrating that body flexibility is not a prerequisite for maneuverability and control when using high aspect ratio foils, serve as a powerful inspiration for the design of underwater vehicles. As observed in [33] and [74], the fore limb kinematics of sea turtles in steady forward swimming are highly asymmetric. The upstroke can take twice as long as the downstroke to complete, and there is significant limb motion inline with swimming direction; as the fore limbs are pulled back along the body during the downstroke, and pushed forward against the flow during the upstroke.

Sea turtle morphology is such that the forelimbs can produce much more torque in 
the down stroke than the upstroke: juvenile turtles are barely capable of raising their limbs out horizontally from the shoulder when held in air [33]. Steady swimming in turtles typically consists of a powerered, high angle of attack downstroke generating forward thrust and maneuvering forces, followed by a much more feathered upstroke, as noted in [74]. Direct observation of turtles at the New England Aquarium with the goal of informing the design of underwater vehicles equipped with flapping foils, particularly Finnegan the RoboTurtle (see [73] and [43]) also show that there can be a significant anterior-posterior motion to high aspect ratio oscillating foils during transient maneuvering behaviors.

\subsection{Oscillating Foil Characterization}

A large body of work exists which focuses on understanding flapping foil actuation, examining wake patterns, force production and efficiency of individual foil actuators in steady flow, using both experimental measurements with mechanically actuated foils, and numerical simulations. The reader is directed to [63] for a survey of work in this field.

Oscillating foils can be characterized by the number of degrees of freedom of the actuation. Single degree of freedom foils are typically twisted about an axis located between the leading edge and the foil centerline. This action is capable of producing thrust above a critical frequency determined by the foil geometry and twisting amplitude. Single degree of freedom foils have been successfully deployed as actuators for underwater vehicles [45].

Foils with two degrees of freedom supplement the twisting motion with a gross periodic motion of the foil, either with angular rotation about an axis perpendicular to the twist axis and parallel to the incoming flow, or with a linear motion along a direction perpendicular to both twist axis and flow direction. The authors have successfully used foils with two degrees of freedom to actuate controlled swimming of an autonomous underwater vehicle, as detailed in [73] and [43].

To date, tests on foils with three or more degrees of freedom have been limited to 
low aspect ratio foils more closely modeled on fish pectoral fin swimmers. In the case of [34] the third degree of freedom is an angular motion which drives the foil forwards and backwards in the direction of the oncoming flow. Additional degrees of freedom can include bending of compliant foils along the span [40]. .

\subsection{Methods}

\subsubsection{Coordinate Definition}

For the purposes of the experiments detailed, an apparatus capable of twisting a vertically oriented foil about its spanwise axis, and moving it in two degrees of freedom in the horizontal plane was placed on a linearly constrained rolling platform above a still water tank, with the foil piercing the free surface of the water. See Figure 6-6 and Figure 6-7.

The foil position is fully described relative to the moving frame of the platform by the co-ordinates $(x, y, \theta)$, where the $Y$ direction is perpendicular to the incoming flow and perpendicular to the foil rotational axis. The positive $X$ direction is parallel to, and opposed to, the incoming flow. The twist angle, $\theta$ is the rotation about the vertical axis, where $\theta=0$ when the foil chordwise centerline is parallel to the incoming flow with the leading edge oriented into the flow. See Figure 6-8 for a schematic with co-ordinate frame indicated.

\subsubsection{Adaptation of Kinematic Parameters}

Experiments with foils oscillating with heave and twist motion are typically characterized by four non-dimensional numbers, which are:

- $R e=\frac{U c}{\mu}$, Reynolds number, defined with respect to chord length, $c$, using incoming flow speed, $U$, and kinematic viscosity.

- $S t=\frac{2 h_{0} f}{U}$, Strouhal number, defined using heave excursion, $2 h_{0}$ as an estimate of wake width, and the oscillating frequency, $f[\mathrm{~Hz}]$, and incoming flow speed, 


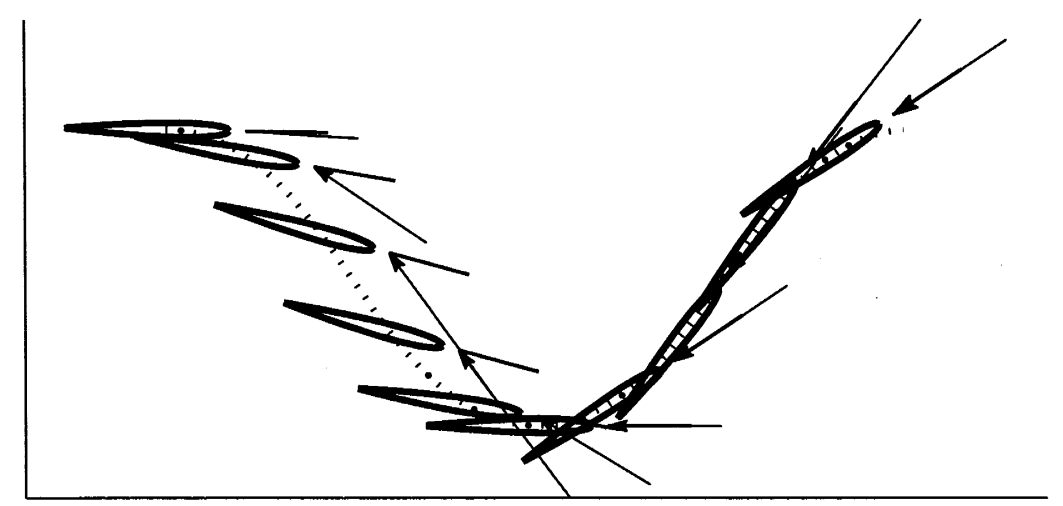

Figure 6-1: Foil position with respect to fluid at regular intervals, where advance angle $\theta_{A D V}=70^{\circ}$. Arrow represent relative flow velocity. The nominal angle of attack is shown by the angle between the relative flow and the line extending the centerline of the foil.

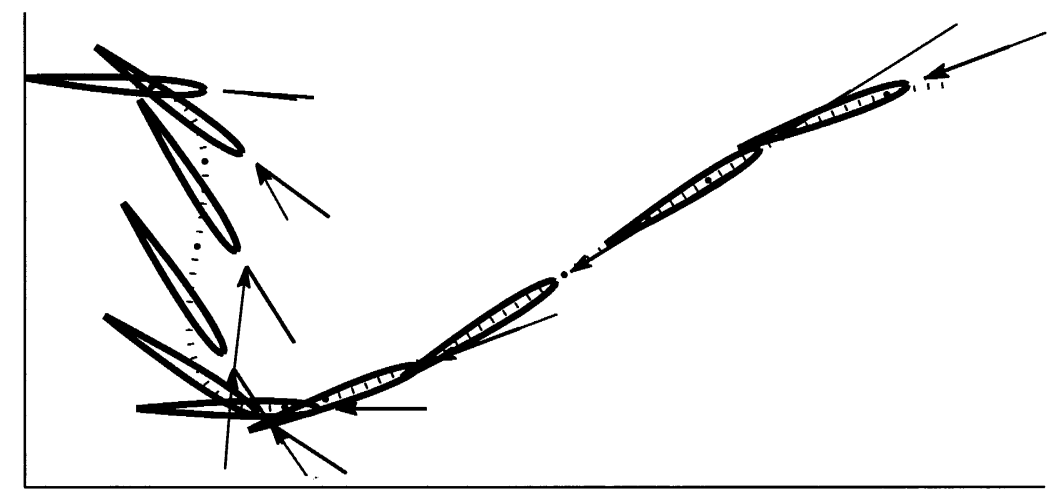

Foil position

with respect to fluid at regular intervals, where advance angle $\theta_{A D V}=100^{\circ}$. Arrow represent relative flow velocity. The nominal angle of attack is shown by the angle between the relative flow and the line extending the centerline of the foil. 


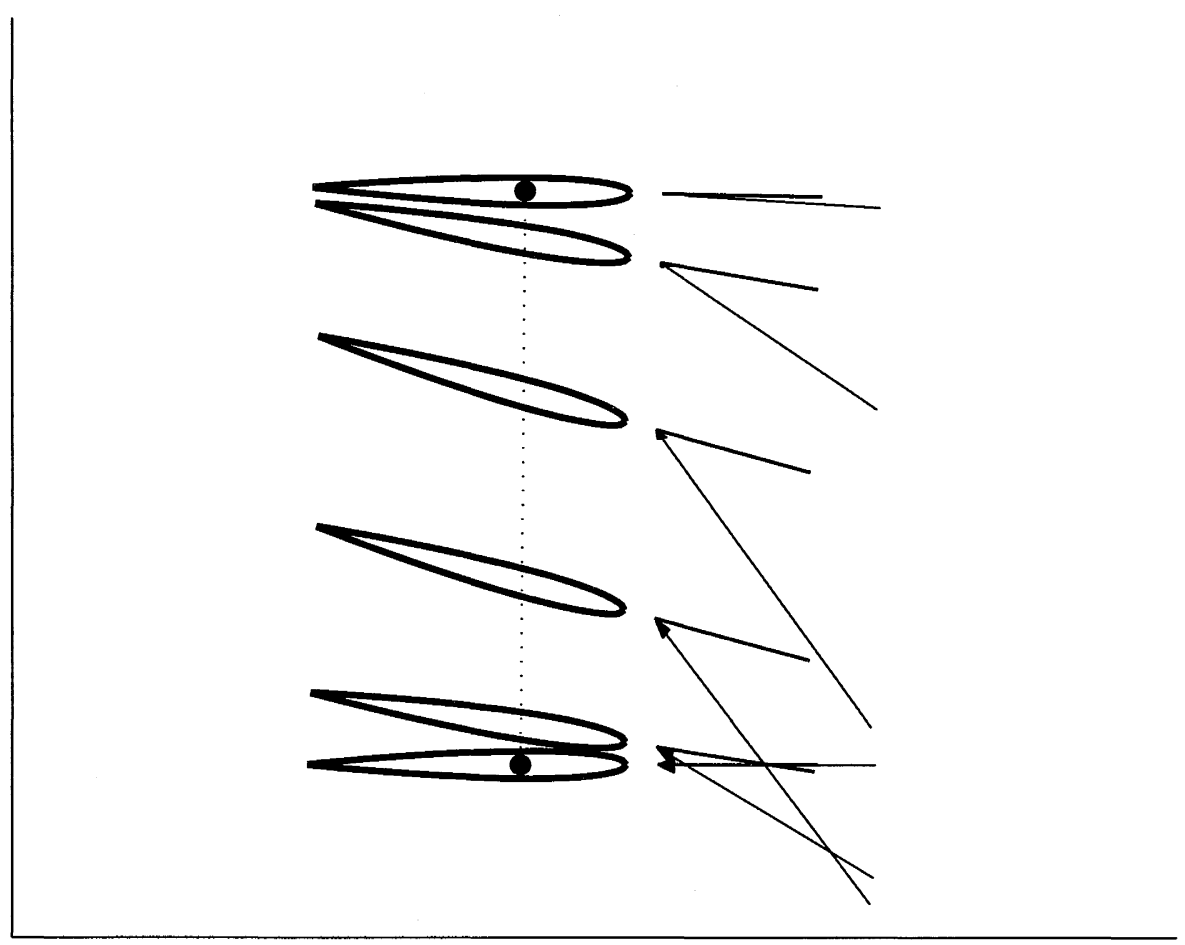

Figure 6-2: Foil position with respect to steadily moving platfrom, at regular intervals, for a downstroke where advance angle $\theta_{A D V}=70^{\circ}$. Arrow represent relative flow velocity. The nominal angle of attack is shown by the angle between the relative flow and the line extending the centerline of the foil.

$U$.

- $\frac{h}{c}$, ratio of heave amplitude to chord length

- $\alpha_{\max }$, maximum nominal angle of attack relative to the foil, where the $\alpha(t)$ is a function of foil velociyt

For the purposes of this study, we divide the foil motion into a downstroke and an upstroke, where the downstroke is the motion of the foil from the point of maximum $+\mathrm{y}$ excursion to the next maximum -y excursion. We can now define four new parameters representing stroke asymmetries:

- $A_{X / Y}=\frac{A_{X}}{A_{Y}}$ the ratio of the in-line ampltiude, $A_{X}$, and transverse amplitude, 


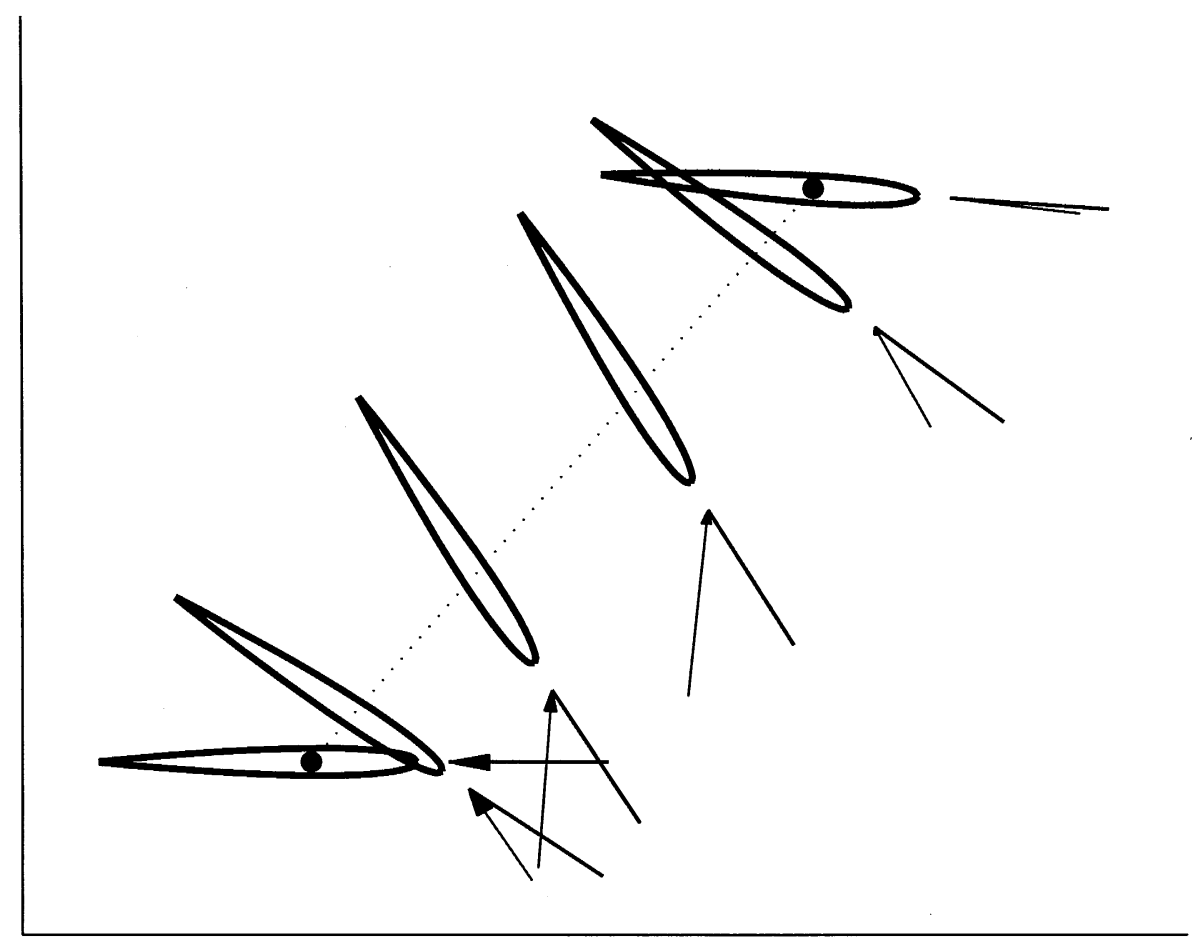

Figure 6-3: Foil position with respect to steadily moving platfrom, at regular intervals, for a downstroke where advance angle $\theta_{A D V}=100^{\circ}$. Arrow represent relative flow velocity. The nominal angle of attack is shown by the angle between the relative flow and the line extending the centerline of the foil.

$A_{Y}$,of the foil, where the excursion is measured relative to the constantly moving frame. Positive values are analogous to the pulling backward of a turtle's forelimb with respect to the body during forward swimming.

- $T_{U / D}=\frac{T_{U}}{T_{D}}$, the ratio of upstroke duration, $T_{U}$, to downstroke duration, $T_{D}$. Increasing values indicate a faster downstroke followed by a slower upstroke, typical of turtle locomotion.

- $\alpha_{\max , U}, \alpha_{\max , D}$, the maximum nominal angle of attack defined above must be replaced with two parameters, the maximum angle of attack of the foil during the upstroke, and of the foil during the downstroke. 


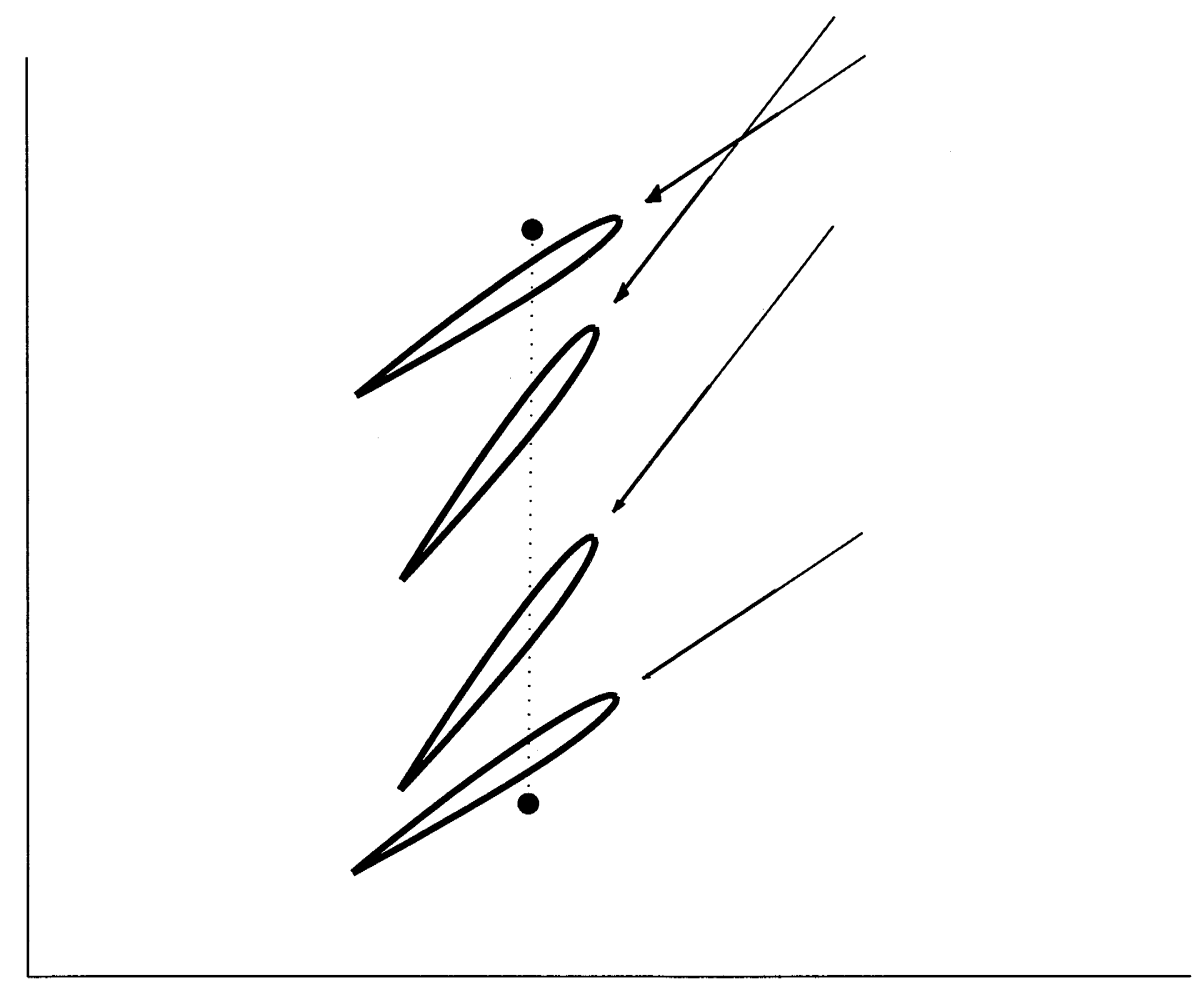

Figure 6-4: Foil position with respect to steadily moving platfrom, at regular intervals, for an upstroke following a downstroke where advance angle $\theta_{A D V}=70^{\circ}$. Arrow represent relative flow velocity. The nominal angle of attack is shown by the angle between the relative flow and the line extending the centerline of the foil.

With the addition of asymmetry between upstroke and downstroke, the existing parameters need to be carefully defined to make their meaning clear. The Strouhal number is a wake parameter, and as the $2 h_{0}$ term in the definition for a transversely heaving foil is intended to approximate the length scale of the wake width, it is appropriate to continue to use transverse amplitude in the definition, i.e.:

- $S t=\frac{2 A_{Y}}{U}$

However, this definition comes with a caveat, which is that the direct relationship between St number and maximum foil velocity and acceleration, which obtains when the incoming velocity is held constant, is severed when inline motion is introduced. A seemingly attractive alternative definition, where the St number is proportional to 


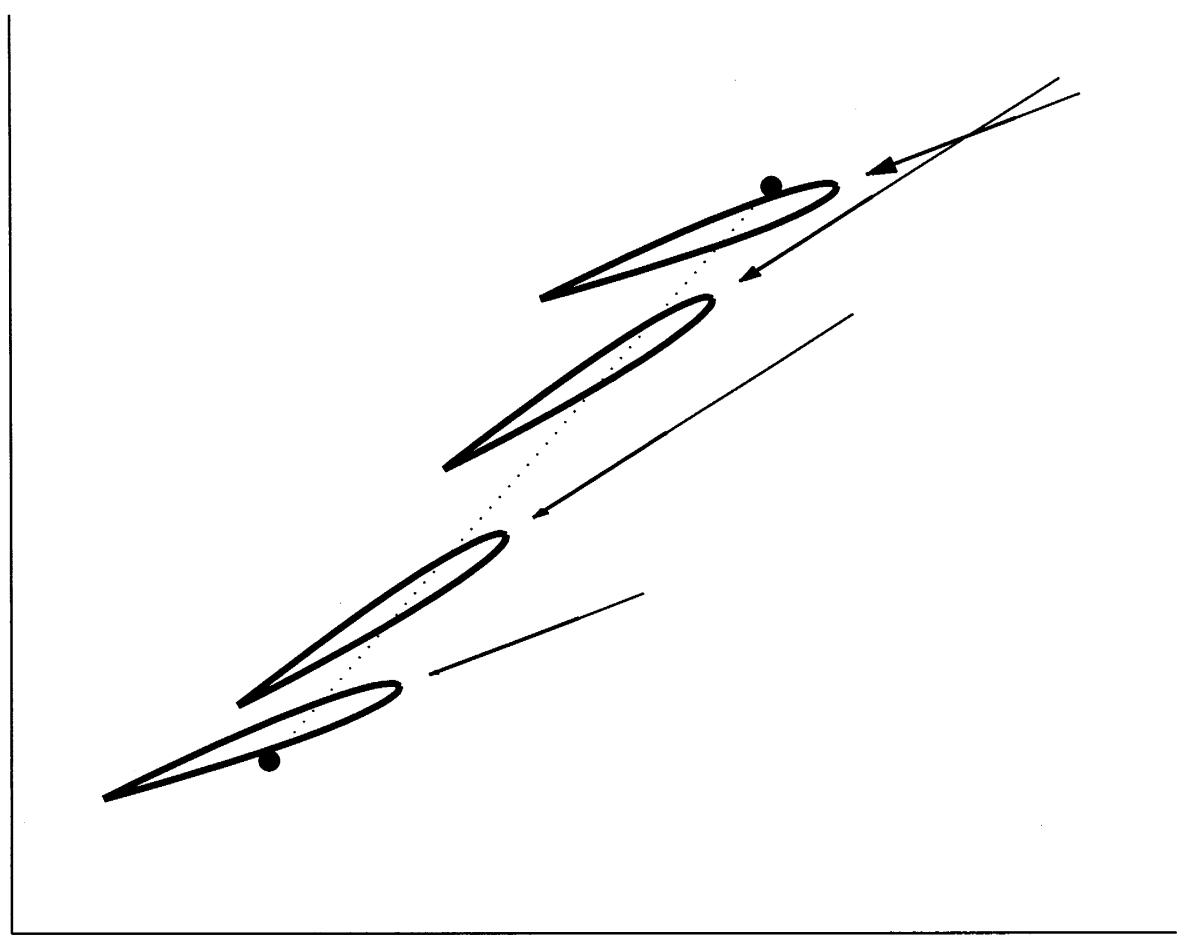

Figure 6-5: Foil position with respect to steadily moving platfrom, at regular intervals, for an upstroke following a downstroke where advance angle $\theta_{A D V}=70^{\circ}$. Arrow represent relative flow velocity. The nominal angle of attack is shown by the angle between the relative flow and the line extending the centerline of the foil.

the total linear amplitude, loses the sense of the St number as a wake parameter.

A further parameter with a useful geometric meaning can be derived from $A_{X / Y}$, $T_{U / D}$ and St. The advance angle, $\theta_{A D V}$, relates the path of the foil relative to the fluid during the middle of the downstroke, or power stroke, which results from the combination of steady platform translation and the relative transverse and inline motions. Where the amplitude of the inline motion, $A_{x}$, is zero, the foil advance angle is minimized for the given kinematics. Increasing $A_{x}$ increases the advance angle (the maximum advance angle for given kinematics is determined by the range of motion of the experimental apparatus in the inline direction.) Where the advance angle is $\pi / 2$, the velocity of the foil shaft with respect to the fluid is perpendicular to carriage velocity at the midpoint of the downstroke. 
Finally, the instantaneous nominal angle of attack of the foil is defined as the angle of attack that results from the instantaneous relative velocity of the foil with respect to the fluid, and the twist angle of the foil:

$$
\alpha(t)=-\arctan \left(\frac{\dot{y}(t)}{U+\dot{x}(t)}\right)+\theta(t)
$$

\subsubsection{Parametric Study}

The following parameters were held constant for all asymmetric foil motions detailed below: $\alpha_{\max , U}=0^{\circ}, \alpha_{\max , D}=40^{\circ}, \frac{h_{0}}{c}=\frac{A_{Y}}{c}=0.9$ and $\frac{T_{U}}{T_{D}}=1$. For comparison purposes, force production using symmetric foil motion was measured for each Strouhal number tested with the same constant parameters with the exception of the upstroke angle of attack, which was set to $\alpha_{\max , U}=\alpha_{\max , D}=40^{\circ}$.

The parameter choices were designed to focus on the potential to improve maneuvering thrust production through the introduction of freedom in the inline direction, given a severe restriction on the actuator power available on the upstroke. The downstroke angle of attack, $\alpha_{m a x, D}$, of $40^{\circ}$ has been shown to be effective in producing high thrust (despite poor efficiency) in symmetrically twisting and heaving foils [55]. The upstroke angle of attack, $\alpha_{\max , U}$ is set to zero to minimize upstroke power, mimicking a completely feathered recovery stroke. Transverse motion was set to take advantage of the the maximum available travel of the apparatus.

Two parameters were varied:

- $S t$ through variation of $f=1 /\left(T_{D}+T_{U}\right)$

- $\theta_{A D V}$ through variation in $A_{X}$

$S t$ ranged from $[0.2,0.6]$ in increments of 0.1. $\theta_{A D V}$ was varied by increments of $10^{\circ}$ from the minimum possible for the given $S t$ (i.e. motion with $A_{X}=0$ ) to the maximum allowed by the apparutus. Given values for all other parameters, an iterative procedure was followed to determine the twist angle $\theta(t)$, transverse position $x(t)$, and relative inline postion $y(t)$ which would result in (half period) sinusoidal 
angle of attack profiles with the desired amplitudes on both the upstroke and the downstroke, while producing the desired $\theta_{A D V}$. A sinusoidal angle of attack is shown to be effective in producing high thrust and hydrodynamic efficiency with heaving and twisting foils in [55] and [27].

The trajectory of the foil is constructed following a modification of the procedure outlined by [27], which imposes a requirement that the nominal angle of attack be sinusoidal in time. In [27], the twist position is required to be sinusoidal in time. For cases with both inline and transverse motion, we modify the procedure by imposing the additional constraint that the foil motion relative to the constantly moving frame of the support platform is always along the same line. For the experiments below, the advance angle is given, hence the velocity of the foil with respect to fluid is known at the point where $\theta(t)=\theta_{\max }$ and $\alpha(t)=\alpha_{\max }$. The twist amplitude is then fully defined using Eq. 6.1, and the twist angle as a function of time is known. For a given direction of relative motion, the linear speed required to produce the desired $\alpha(t)$ is a function only of the twist amplitude imposed, as all other parameters are given. The angle of the motion relative to the platform is varied until the total transverse motion of the foil (as calculated by numerically integrating the velocity) is equal to the value given by the desired $\frac{h}{c}$.

The procedure for finding foil kinematics begins with setting $x$ and $y$ to be in phase:

$$
\begin{aligned}
& x(t)=A_{\frac{X}{Y}} y(t) \\
& \dot{x}(t)=A_{\frac{X}{Y}} \dot{y}(t)
\end{aligned}
$$

Define twist, $\theta$, and angle of attack, $\alpha$, to be piecewise sinusoidal. For the downstroke:

$$
\begin{aligned}
\alpha(t) & =\alpha_{0}^{D} \sin (\omega t) \\
\theta(t) & =\theta_{0}^{D} \sin (\omega t)
\end{aligned}
$$


For a given $\alpha_{0}^{D}$ and advance angle, $\theta_{A D V}$, we can easily calculate $\theta_{0}^{D}$ from the geometry at the midpoint of the downstroke:

$$
\theta_{0}^{D}=\alpha_{0}^{D}-\theta_{A D V}
$$

With given $\alpha_{0}^{D}$, we have fully defined $\alpha(t)$ and $\theta(t)$ for the downstroke using (6.3) and (6.4). For any value of $A_{\frac{X}{Y}}$, we can now calculate $\dot{x}(t)$ and $\dot{y}(t)$ from (6.2) and the relation:

$$
\alpha(t)=-\arctan \left(\frac{\dot{y}(t)}{U+\dot{x}(t)}\right)+\theta(t)
$$

The total excursion of the foil over the downstroke, $\Delta y_{D}=\int_{0}^{\frac{T}{2}} \dot{y} d t$, is a function of the one free variable, i.e. $\Delta y_{D}=\Delta y_{D}\left(A_{\frac{X}{Y}}\right)$. We can numerically solve for $A_{\frac{X}{Y}}$ such that $\Delta y_{D}\left(A_{\frac{X}{Y}}\right)-\Delta y_{d}=0$, where $y_{d}$ is the desired transverse amplitude set by $\frac{h_{0}}{c}$.

On the upstroke, $\alpha(t)$ and $\theta(t)$ are once again sinusoidal, but now the value of $A_{\frac{X}{Y}}$ required to recover from the downstroke is fixed, and $\theta_{0}^{U}$ is no longer constrained by $\theta_{A D V}$ (i.e. there is no upstroke equivalent to (6.4).) $\theta_{0}^{U}$ is the free variable which determines the total excursion of the foil over the upstroke: $\Delta y_{U}=f\left(\theta_{0}^{U}\right)$, and we can solve for $\theta_{0}^{U}$ such that $\Delta y_{U}\left(\theta_{0}^{U}\right)-\Delta y_{d}=0$.

The final result is a piecewise sinusoidal twist angle, with the transverse and inline motion calculated to satisfy the requirements for piecewise sinusoidal angle of attack with given amplitude, and for given transverse amplitude and advance angle.

The resulting foil motion is illustrated in detail for two cases with different advance angles in Figure 6-1 through Figure 6-5. In the first case, $\theta_{A D V}=69^{\circ}$, the minimum possible advance angle for the given parameters, achieved with no relative inline motion during the downstroke. In the other, $\theta_{A D V}=110^{\circ}$, such that the X-component of the absolute velocity of the foil is of opposite of the velocity of the platform at the center of the downstroke. The difference between the two cases is illustrated in Figure 6-1 and Figure 6.3.2, where the position and orientation of the foil is shown 


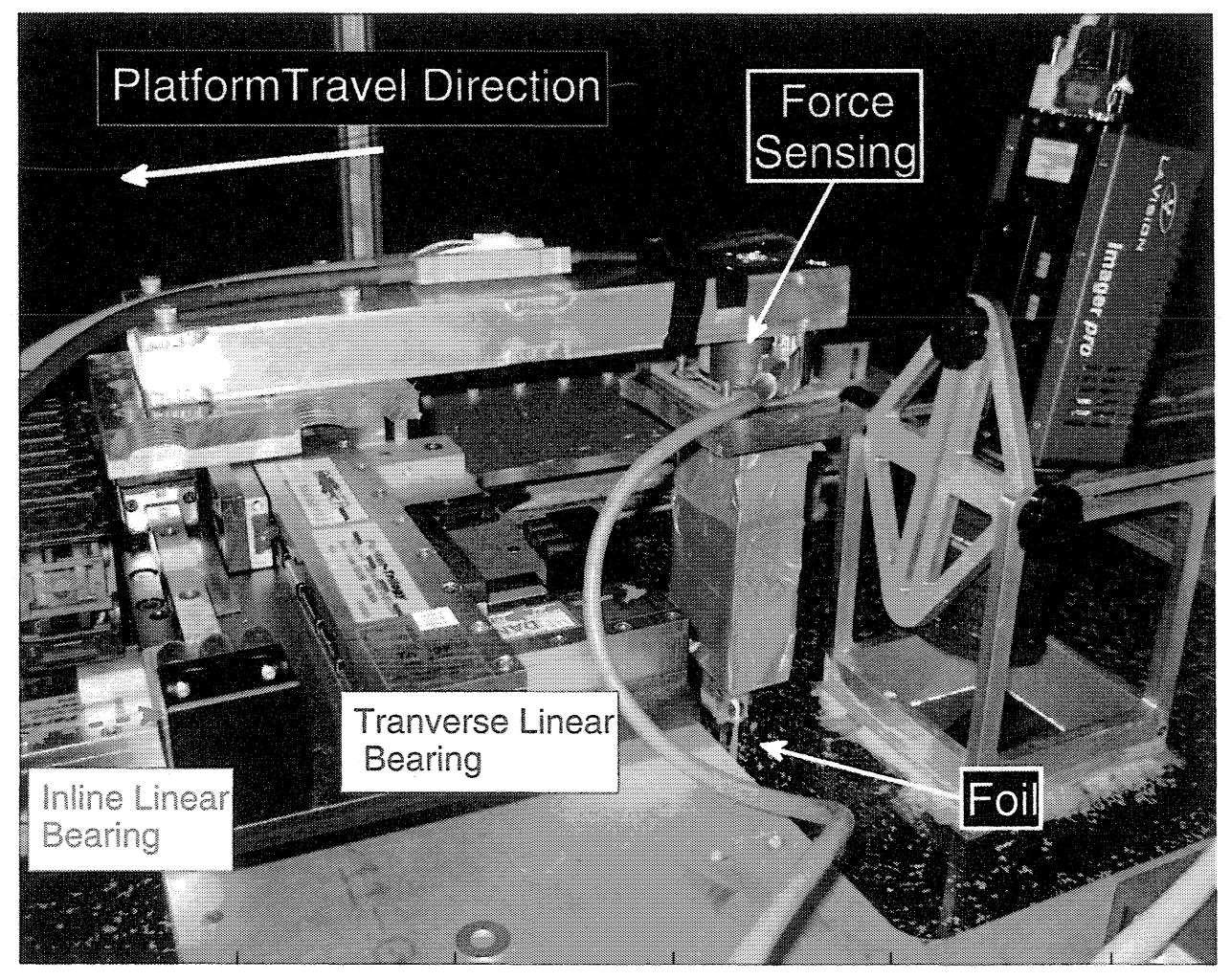

Figure 6-6: View of single foil experimental apparatus showing actuators, sensor and foils mounted to moving platform.

at regular time intervals in the stationary frame (i.e. the frame of the water tank.) Figure 6-2 and Figure 6-3 offer a more detailed view of the resulting nominal angle of attack of the foils during the downstrokes, while illustrating the motion of the foil that is actuated with respect to the moving platform. Figure 6-4 and Figure 6-5 show the same for the upstrokes. Note that in the case where the minimum advance angle is used, there is no inline motion of the foil with respect to the platform.

The foil used for this study was a length of extruded aluminum with a NACA-012 cross-section, with no camber and a constant chord of $6.93 \mathrm{~cm}$. The foil was clamped at one end to the shaft of a small servo motor, with the axis of rotation a distance of $1 / 3$ of the chord length behind the leading edge. The foil pierced the free surface of the water and extended $0.53 \mathrm{~m}$ below the surface, where it terminated with a square end.

The foil bearing structure was mounted to the load side of a 6 -axis load cell from JR3, Inc., with a linear load capacity of $110 \mathrm{~N}$ and moment capacity of $220 \mathrm{~N}-\mathrm{m}$. 


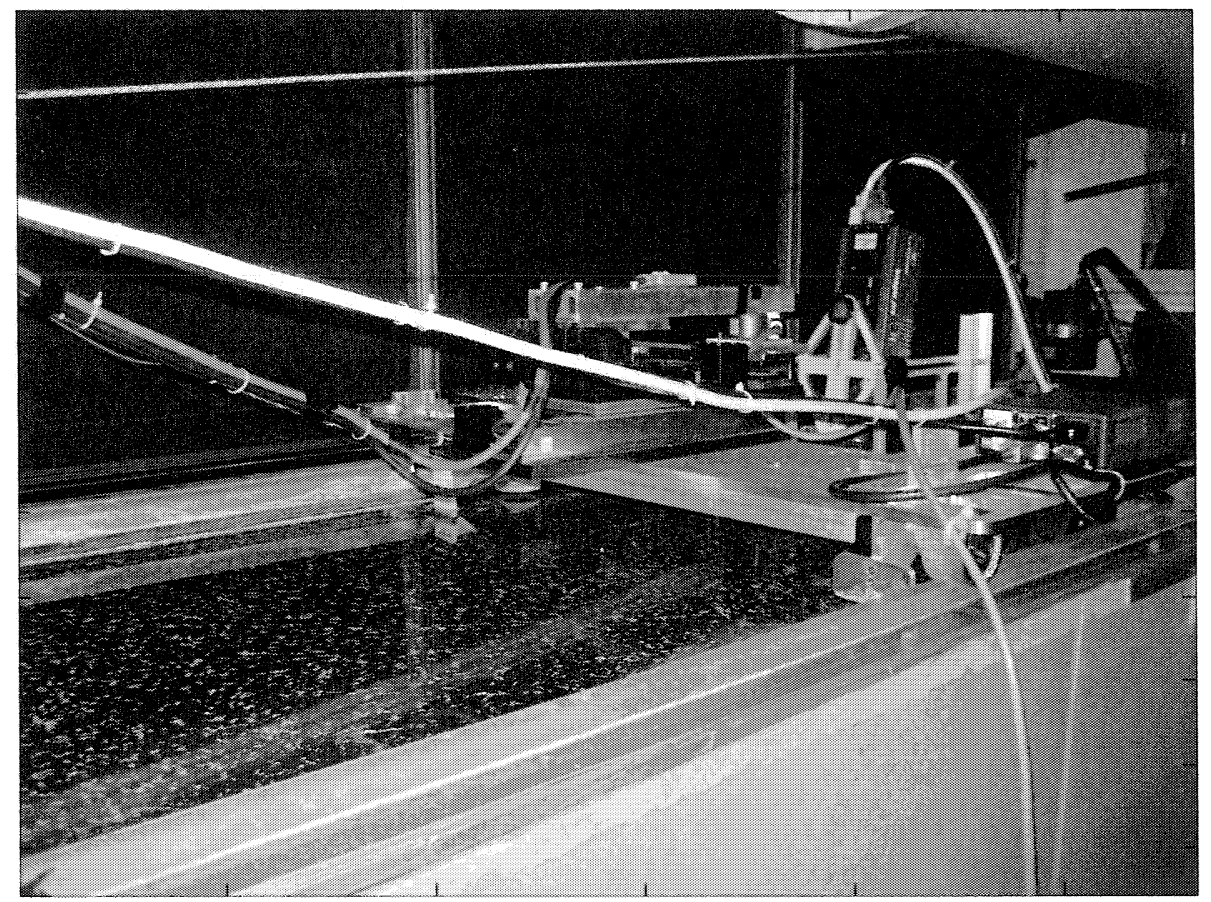

Figure 6-7: View of single foil experimental apparatus showing position of moving platform on water tank.

The base of the force sensor was attached to the end of a cantilever beam, which was driven in the horizontal plane by a pair of linear servo motors with $0.18 \mathrm{~m}$ travel. Amplified sensor output was differentially captured captured at $1 \mathrm{kHz}$ with a National Instruments USB-6211 DAQ card. The foil drive motors were mounted to a rolling platform above an $0.75 \mathrm{~m} \times 0.75 \mathrm{~m} \times 2.4 \mathrm{~m}$ glass water tank. The test apparatus is pictured in Figure 6-6 and Figure 6-7.

\subsection{Results and Discussion}

\subsubsection{Lift and Thrust for Symmetric vs. Asymmetric Kine- matics}

The mean lift and thrust results from the full set of experiments with St number ranging from 0.2 to 0.6 at all tested advance angles are shown in Figure 6-9. Mean 


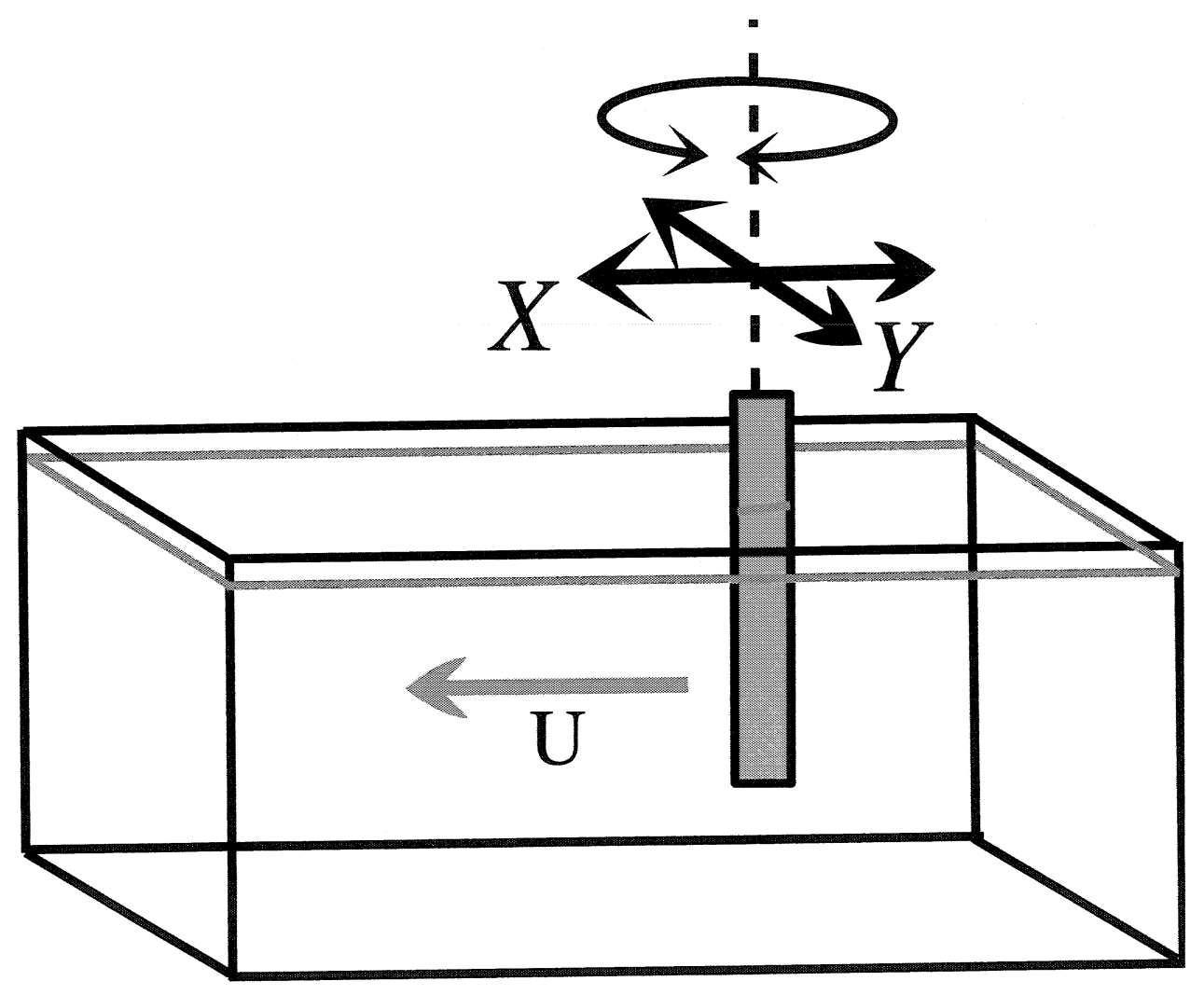

Figure 6-8: Cartoon of single foil experimental apparatus indicating dimensions as well as direction of positive transverse, inline and twist motion with respect to direction of steady translation.

lift and thrust coefficients are calculated as the average over an entire cycle of motion:

$$
\begin{aligned}
& \bar{C}_{L}=\frac{\int_{0}^{T} L(t) d t}{\frac{1}{2} \rho U^{2}} \\
& \bar{C}_{T}=\frac{\int_{0}^{T} T(t) d t}{\frac{1}{2} \rho U^{2}}
\end{aligned}
$$

$\bar{C}_{L}$ and $\bar{C}_{T}$ are calculated in the same manner for both symmetric and asymmetric foil motions. The values of lift and thrust are plotted for each St number as a function of advance angle for the asymmetric kinematics. For each St number, a single point represents $\bar{C}_{T}$ of the symmetric case, plotted against the advance angle resulting from the pure transverse motion (which matches the lowest advance angle of the asymmetric motion.) The loss of thrust that results from altering the symmetric motion to an asymmetric motion by setting the upstroke nominal maximum angle of attack to 


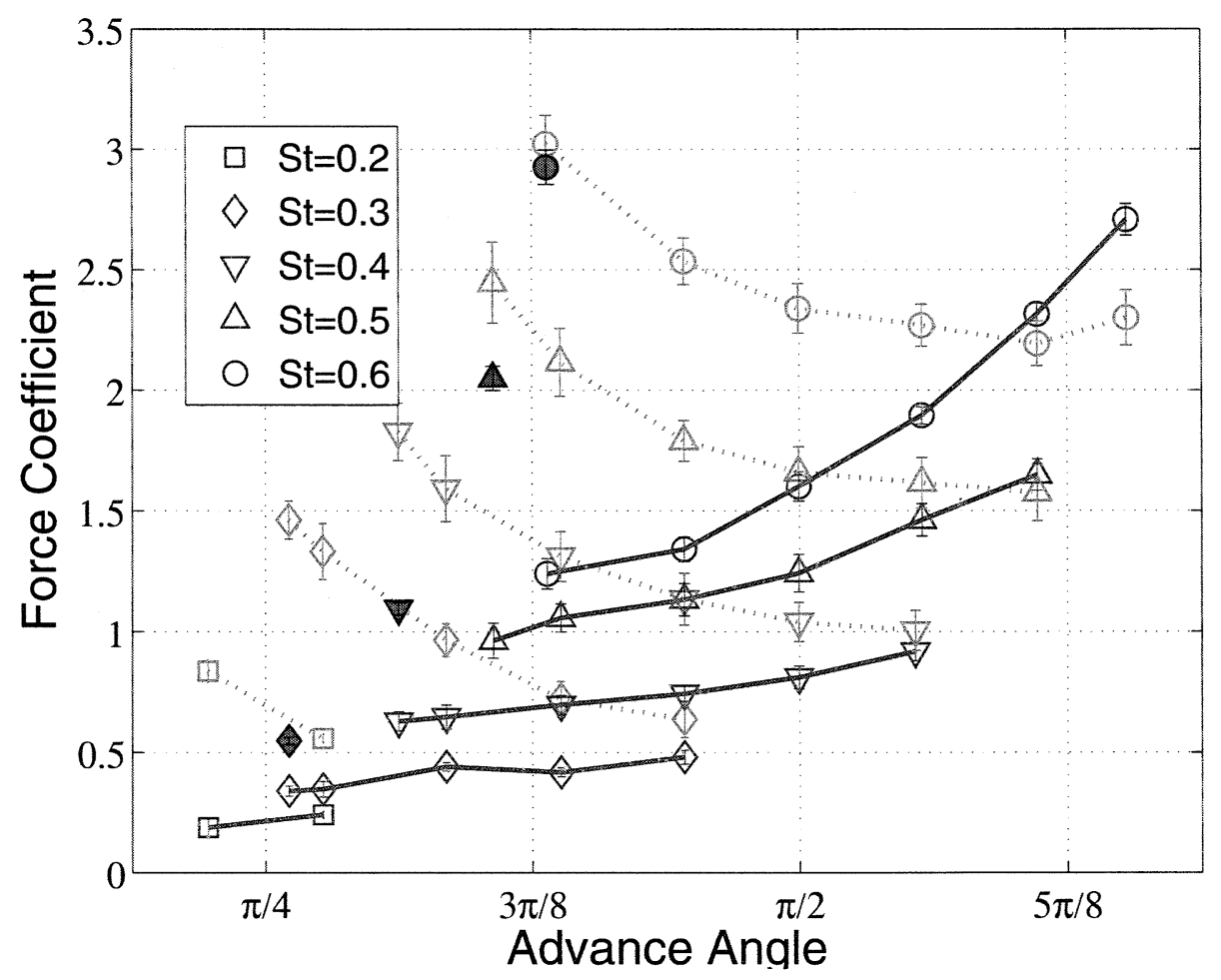

Figure 6-9: Mean lift coefficient, $\overline{C_{L}}$ and mean thrust coefficient, $\overline{C_{T}}$ for asymmetric foil motion with downstroke $\alpha_{\max }=40^{\circ}$, upstroke $\alpha_{\max }=0^{\circ}$, vary Strouhal number and advance angle. Blue: $\overline{C_{T}}$, Red: $\overline{C_{L}}$. Filled symbols indicate $\overline{C_{T}}$ for symmetric motions with no inline motion, and upstroke $\alpha_{\max }=40^{\circ}$.

zero, without adding inline motion, can be immediately seen from comparison of of the symmetric mean thrust values to the mean thrust values at the minimum advance angle at each St number. This drop in thrust moving from a high angle of attack upstroke to a fully feathered (zero angle of attack) upstroke ranges from $42 \%$ ( $\mathrm{St}=$ $0.3)$ to $59 \%(\mathrm{St}=0.6)$.

For every St number tested using asymmetric kinematics, the thrust is monotonically increasing with increasing advance angle, while lift is at a maximum at the lowest advance angle (zero inline motion.) For the higher Strouhal numbers tested the lift reaches a minimum at an advance angle greater than $\pi / 2$, but the minimum occurs at the highest testable advance angle for $\mathrm{St}=[0.2,0.3,0.4]$. Notably, while the total thrust increases by as much $225 \%$ between minimum and maximum advance angle for the best case $(\mathrm{St}=0.6$,$) the thrust generated by asymmetric stroking never$ 


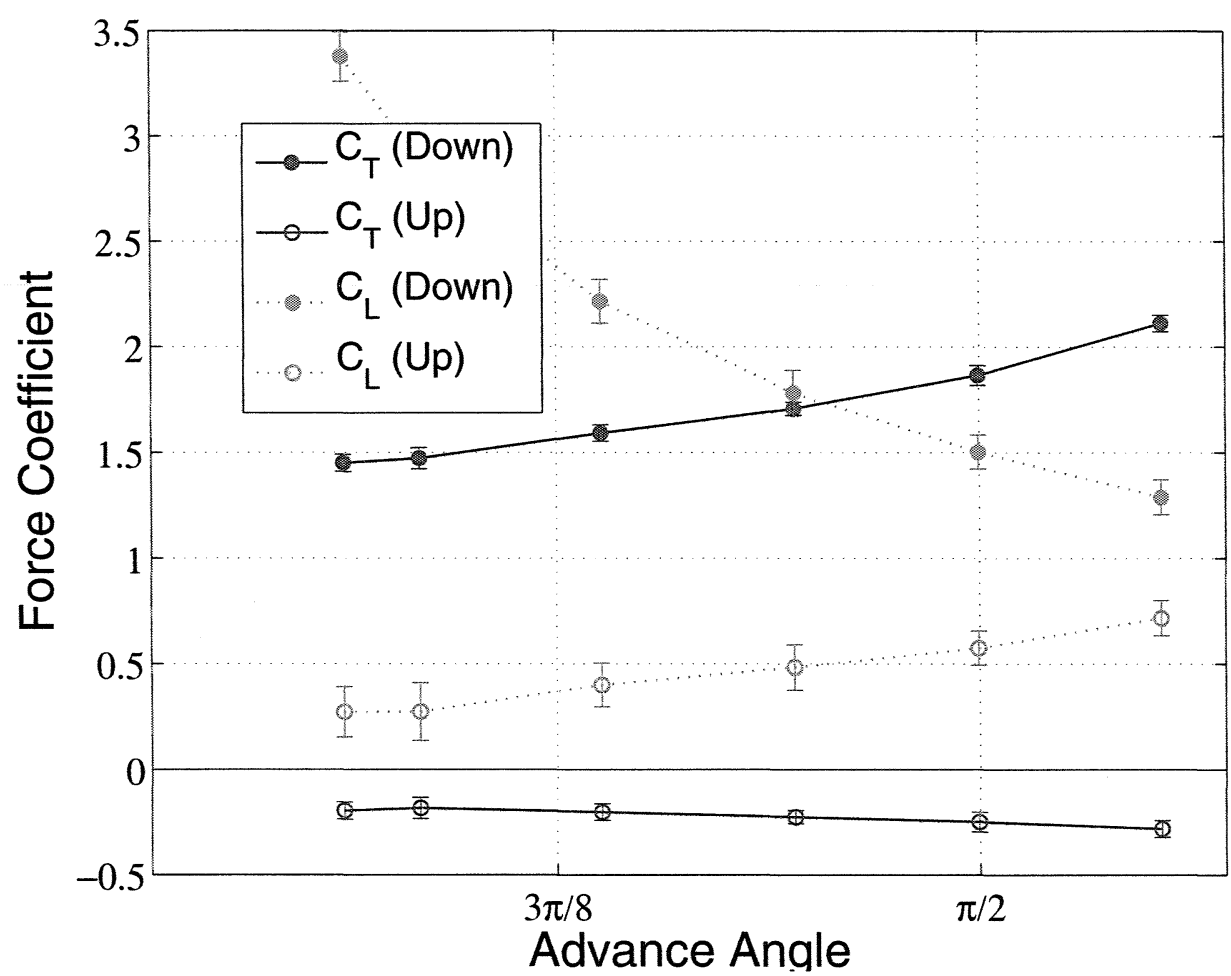

Figure 6-10: Comparison of mean lift coefficient, $\overline{C_{L}}$ and mean thrust coefficient, $\overline{C_{T}}$ over each half stroke for asymmetric foil motion, with downstroke $\alpha_{\max }=40^{\circ}$, upstroke $\alpha_{\max }=0^{\circ}$, Strouhal number $=0.4$, with varying advance angle. Filled symbols indicate $\overline{C_{T}}$ for full cycles of symmetric motions with no inline motion and upstroke $\alpha_{\max }=40^{\circ}$.

reached the level of thrust generated during the symmetric motion.

\subsubsection{Lift and Thrust During Upstroke and Downstroke}

To compare thrust production and lift recorded during the upstroke and the downstroke, Figure 6-10 illustrates the change in $\bar{C}_{L}^{U}, \bar{C}_{L}^{D}, \overline{C_{T}^{U}}$, and $\overline{C_{T}^{D}}$, for all advance angles tested at $\mathrm{St}=0.4$ where:

$$
\begin{gathered}
\overline{C_{L}^{D}}=\frac{\int_{0}^{\frac{T}{2}} L(t) d t}{\frac{1}{2} \rho U^{2}} \\
\overline{C_{L}^{U}}=\frac{\int_{\frac{T}{2}}^{T} L(t) d t}{\frac{1}{2} \rho U^{2}}
\end{gathered}
$$




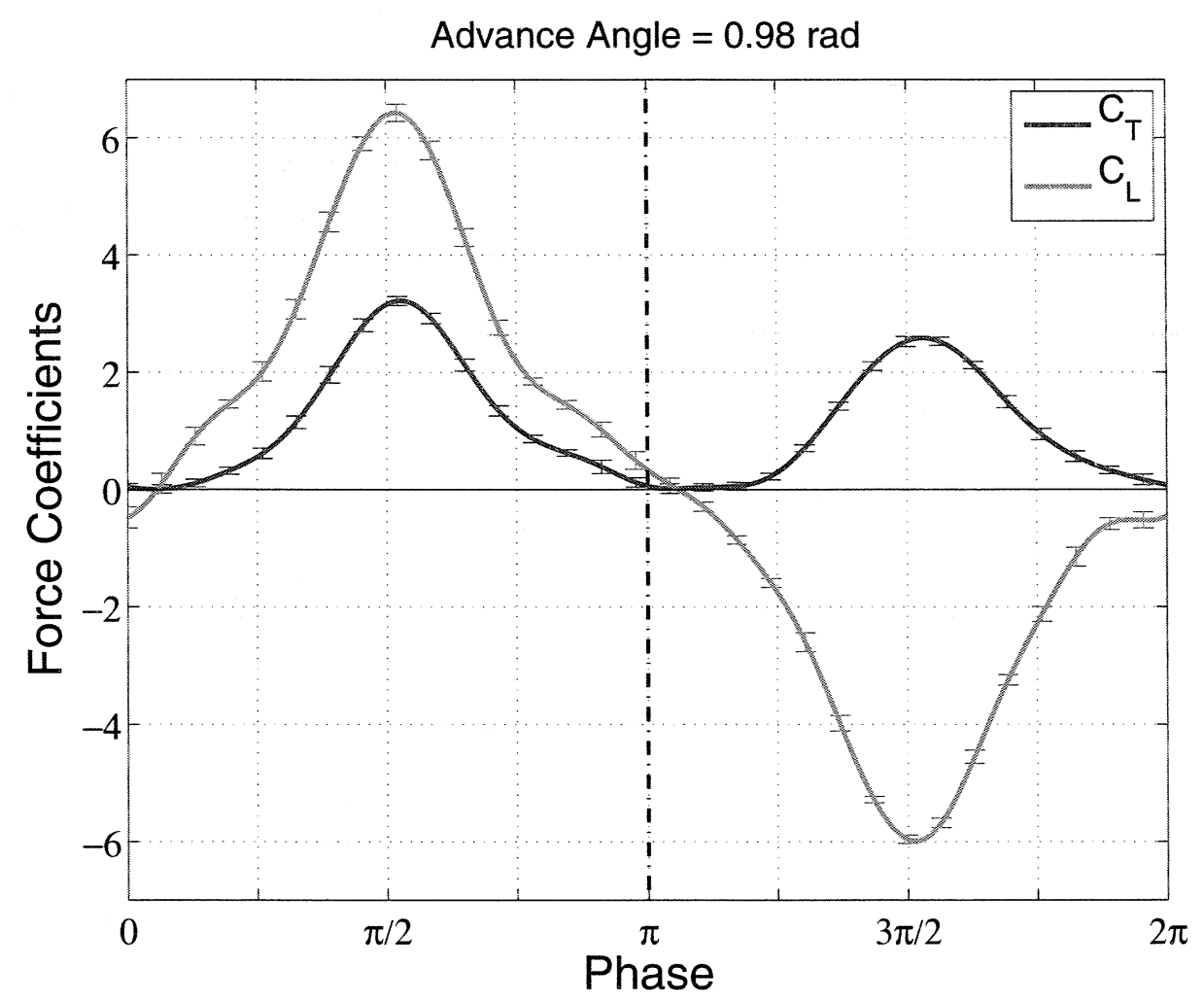

Figure 6-11: Phase averaged thrust coefficient, $C_{T}$ and lift coefficient, $C_{L}$ for symmetric foil motion, with downstroke $\alpha_{\max }=40^{\circ}$, upstroke $\alpha_{\max }=40^{\circ}$, and Strouhal number $=0.4$,

$$
\begin{gathered}
\overline{C_{T}^{D}}=\frac{\int_{0}^{\frac{T}{2}} T(t) d t}{\frac{1}{2} \rho U^{2}} \\
\overline{C_{T}^{U}}=\frac{\int_{\frac{T}{2}}^{T} T(t) d t}{\frac{1}{2} \rho U^{2}}
\end{gathered}
$$

As might be expected if the majority of the effort is expended during the downstroke, the thrust developed during the downstroke increases as the advance angle increases from minimum to maximum, from $\bar{C}_{T}^{D}=1.5$ to $\bar{C}_{T}^{D}=2.1$, and the lift decreases from $\overline{C_{L}^{D}}=3.45$ to $\overline{C_{L}^{D}}=2.1$. The upstroke, on the other hand, generates negative thrust (drag) and positive lift. The magnitude of the drag increases from 0.15 to 0.3 for a change of 0.15 over the $\theta_{A D V}$ range (compared to downstroke thrust production change of 0.6 ) as the nominally feathered foil is swept forward with greater speed to compensate for the larger inline motion during the downstroke required to increase the advance angle. 


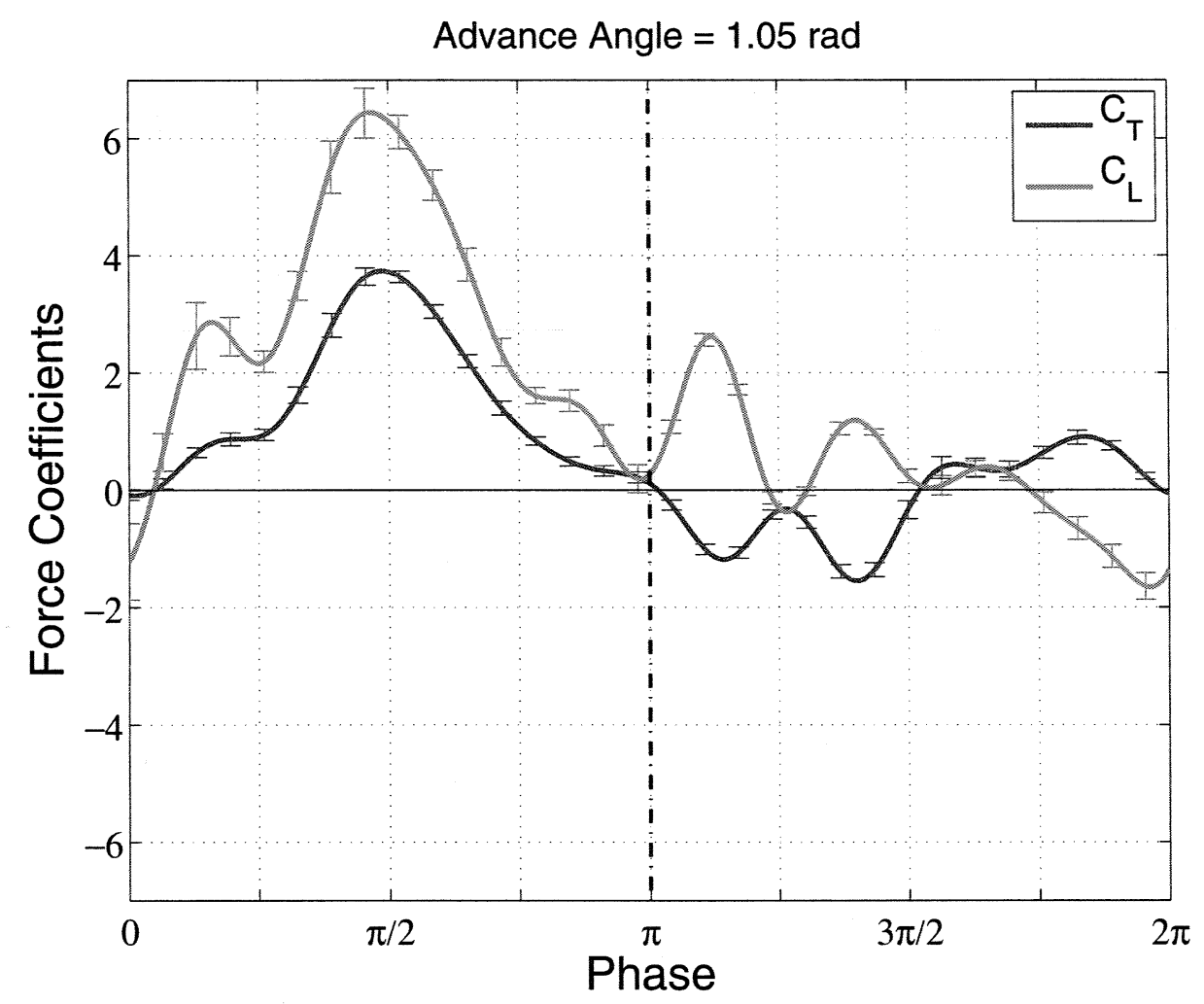

Figure 6-12: Phase averaged thrust coefficient, $C_{T}$ and lift coefficient, $C_{L}$ for asymmetric foil motion, with downstroke $\alpha_{\max }=40^{\circ}$, upstroke $\alpha_{\max }=0^{\circ}$, Strouhal number $=0.4$, with no inline motion relative to the steadily translating carriage

Positive lift for the upstroke indicates that the lift force is in the same direction as the transverse motion of the foil. Intuitively, setting the nominal angle of attack to zero has resulted in the the foil acting as a lifting surface, with small angle of attack, oriented to aid the upward motion of the foil. A free-swimming vehicle or animal using these kinematics would be sacrificing a small amount of speed (dictated by the magnitude of $C_{T}^{U}$ ) to reduce the torque load on the upward actuation or musculature.

The separation between upstroke and downstroke thrust production is inherently artificial, as lift and thrust during each half stroke is directly affected by the induced flow of the previous half stroke, whether in the form of wake patterns or attached vorticity. The magnitude of this effect is brought out by a comparison of Figure 6-11 and Figure 6-12. In the first figure, time traces of thrust and lift, phase averaged from all available experimental cycles, are shown for symmetric foil kinematics with downstroke $\alpha_{\max }=40^{\circ}$, upstroke $\alpha_{\max }=40^{\circ}$, and Strouhal number $=0.4$. As 


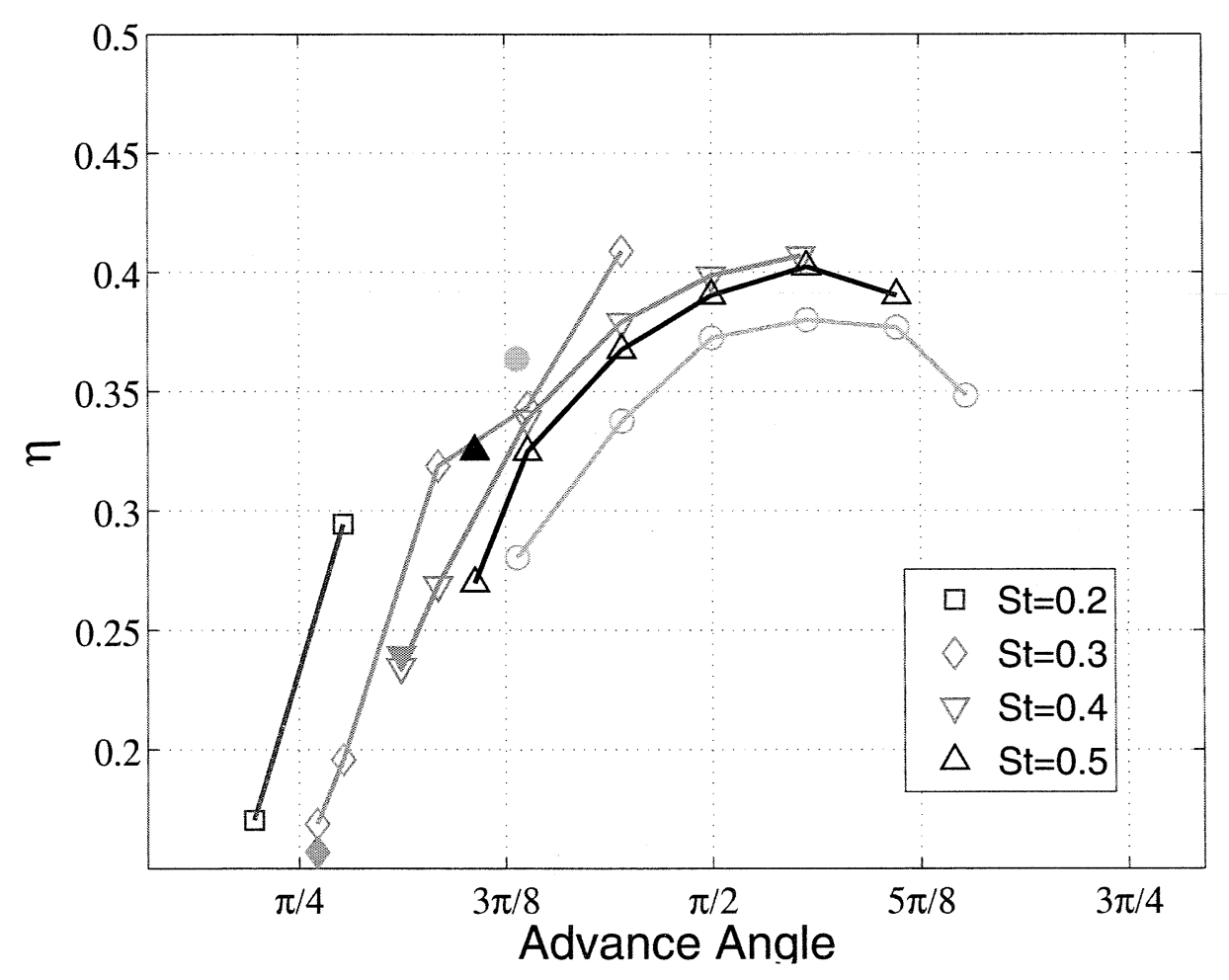

Figure 6-13: Efficiency, $\eta$, of thrust production varying advance angle for foils with downstroke $\alpha_{\max }=40^{\circ}$, upstroke $\alpha_{\max }=0^{\circ}$, for all values of St number tested. Filled symbols indicate $\eta$ for symmetric foil motions, with with downstroke $\alpha_{\max }=40^{\circ}$, upstroke $\alpha_{\max }=40^{\circ}$, and no inline motion, for each value of St number

expected, lift with the same magnitude and shape but opposite sign is produced during the upstroke and during the downstroke. Positive thrust is generated during both the upstroke and downstroke.

In Figure 6-12, positive lift and thrust are again generated during the downstroke, but on the upstroke higher frequency lift and thrust traces with lower magnitude result in the much lower upstroke force coefficients seen in Figure 6-10. The contrast during the downstroke between the single peaked lift trace of the symmetric motion and the multi-peaked lift trace of the asymmetric motion provides a direct measure of the history effect of the upstrokes, as the foil motion is identical during the downstroke. 

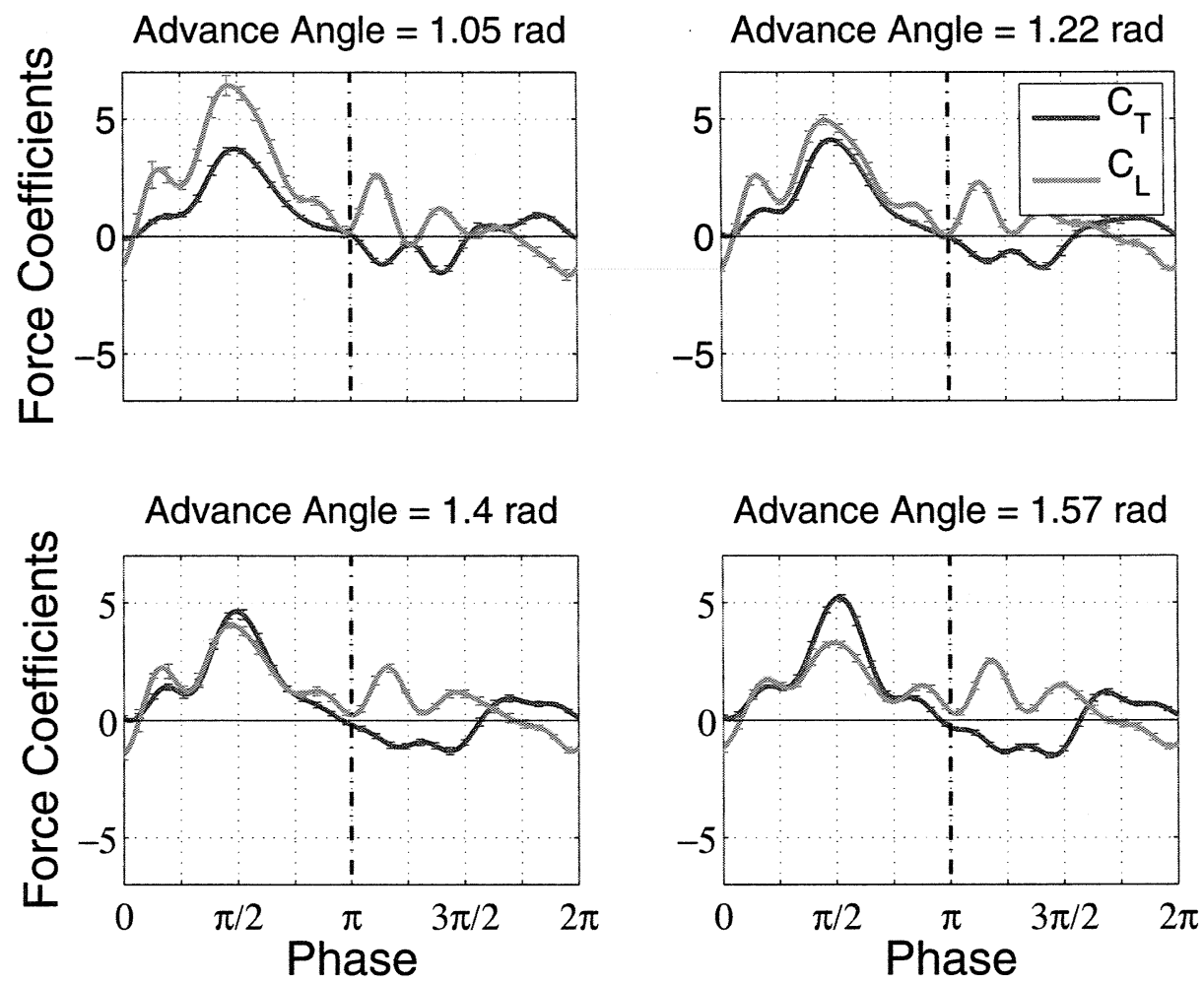

Figure 6-14: Phase averaged thrust coefficient, $C_{T}$ and lift coefficient, $C_{L}$ for downstroke $\alpha_{\max }=40^{\circ}$, upstroke $\alpha_{\max }=0^{\circ}$, Strouhal number $=0.4$, for four cases with advance angles ranging from the minimum possible (i.e. no relative inline motion) to $\pi / 2$, i.e. no forward motion relative to the water in the center of the downstroke.)

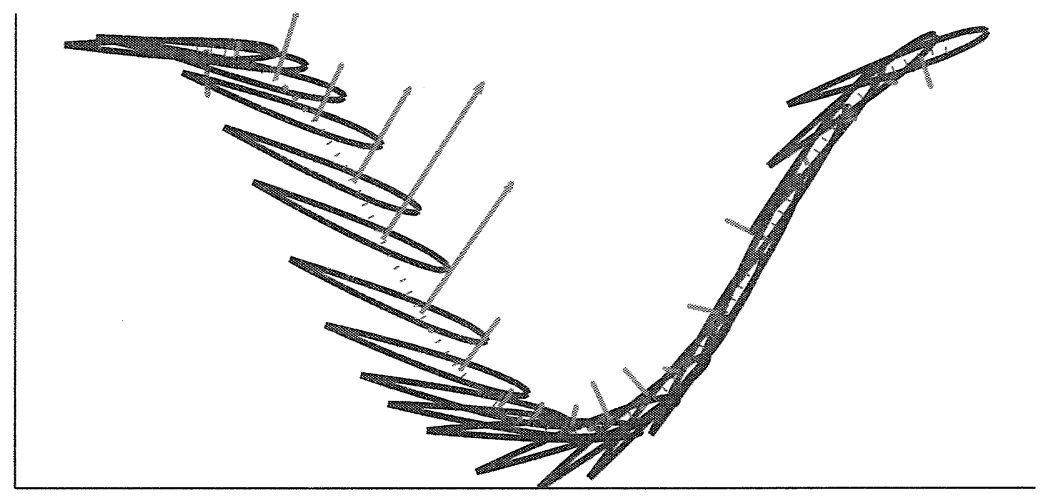

Figure 6-15: Foil position with respect to fluid at regular intervals, where advance angle $\theta_{A D V}=70^{\circ}$. Arrows represent recorded fluid force on foil. 


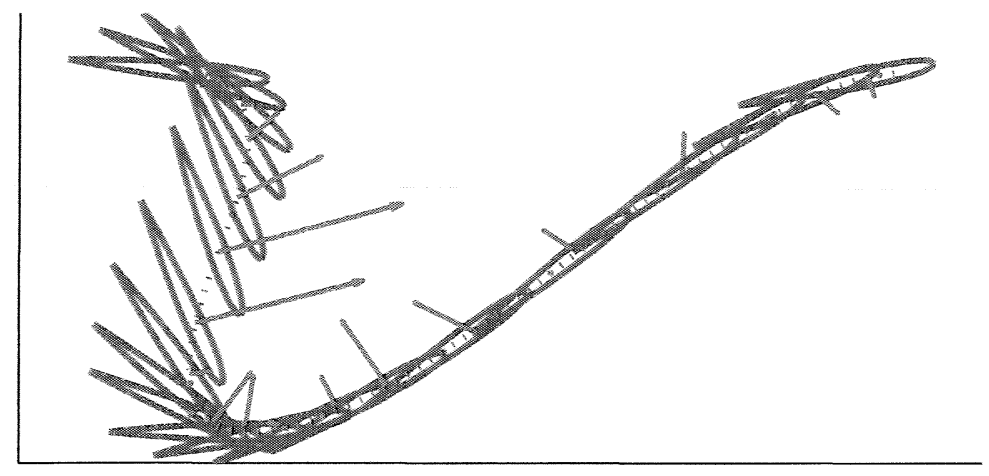

Figure 6-16: Foil position with respect to fluid at regular intervals, where advance angle $\theta_{A D V}=100^{\circ}$. Arrows represent recorded fluid force on foil.

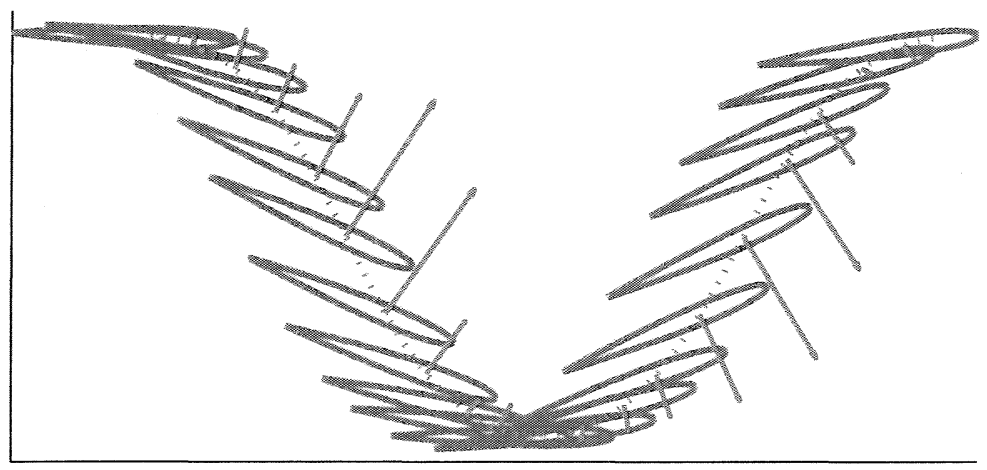

Figure 6-17: Foil position with respect to fluid at regular intervals, where advance angle $\theta_{A D V}=100^{\circ}$. Arrows represent recorded fluid force on foil.

\subsubsection{Rotation of Peak Force Direction with Increasing Inline Motion}

Figure 6-15 through Figure 6-17, show foil position and orientation with respect to the fluid throughout a single cycle of motion, with arrows representing force direction and magnitude, for three cases: symmetric motion with $\mathrm{St}=0.4$, asymmetric motion with no inline motion using a feathered upstroke $\left(\theta_{A D V}=70^{\circ}\right)$, and asymmetric 
kinematics with inline motion and a feathered upstroke $\left(\theta_{A D V}=100^{\circ}\right)$.

The first observation to make is that the while, as discussed above, the downstroke thrust time trace is qualitatively different for the symmetric case versus the asymmetric case with no inline motion, the peak thrust and peak lift occur at the same phase, with the close to the same magnitudes and hence the same direction. Feathering the upstroke has not fundamentally changed the thrust mechanism in such a way as to dramatically reduce the available thrust on the downstroke, or either increase or decrease the (not necessarily desirable) lift forces perpendicular to the travel direction.

Given that nearly the same force is available during the power stroke, the force vector and foil visualizations, taken together with Figure 6-14, illustrate how the thrust is increased at the same time that the lift is decreased by using inline motion. The experimental results show that the rotation of the force vector nearly preserves the force direction with respect to the foil at peak force. When the inline motion is added, the same nominal foil angle of attack can be maintained with much greater foil twist amplitude; as the inline motion increases between cases, the force vector rotates along with the foil, resulting in a more forward orientation.

The rotation of the force direction from possibly unwanted lift force directly opposing the foil motion, to useful thrust force, results in efficiency increases as $\theta_{A D V}$ increases. Efficiency is defined as the work done by the foil on the fluid (i.e. work done by the actuators with the foil and motor inertial contribution removed) as a fraction of the useful work (i.e. force in the direction of motion multiplied by the forward velocity:

$$
\begin{aligned}
P(t) & =(\dot{x(t)}, \dot{y(t)}) \cdot\left(F_{x}(t), F_{y}(t)\right) \\
\eta & =\frac{U \int_{0}^{T} F_{x}(t) d t}{\int_{0}^{T} P(t) d t}
\end{aligned}
$$

Note that twist motor power input is assumed to be negligible for this discussion. From Figure 6-13, where $S t=[0.2,0.3,0.4]$, efficiency increases going from the 
kinematics with no inline motion to kinematics where the maximum $\theta_{A D V}$ attained, although the slope $\frac{\partial \eta}{\partial \theta_{A D V}}$ deceases with increasing $\theta_{A D V}$. For $S t=[0.5,0.6], \eta$ peaks at $90^{\circ}<\theta_{A D V}<110^{\circ}$, approximately where the decline in mean lift is reversed as seen in Figure 6-9. (Note that $\bar{C}_{L}$ does not provide any information about lift as a function of foil phase, and thus efficiency cannot be obtained solely by examining $\bar{C}_{L}$ and $\bar{C}_{T}$. The coincidence of minimum of $\bar{C}_{L}$ and maximum $\eta$ where $S t=0.6$ should not be taken to indicate that the differing $\theta_{A D V}$ at minimum $\bar{C}_{L}$ and maximum $\eta$ for $\mathrm{St}=0.5$ is the result of an error.)

It is important to note that the addition of inline motion here, certainly up to an advance angle of $90^{\circ}$ cannot be considered to change the basic thrust stroke from a lift based to a drag based or rowing stroke, as the foil is not moving against the direction of travel, and the resulting thrust is primarily oriented perpendicular to the foil direction of motion during the thrust production stage, rather than parallel.

\subsection{Conclusion}

We should not naively assume that the asymmetric torque capability of sea turtle forelimbs is driven by the requirement for more effective swimming. The defensive advantages of a hard shell, the requirement for female turtles to drag themselves up a beach and dig nests, and perhaps other functional requirements for turtles to succeed in their environment all may outweigh the need to optimize propulsion, whether in terms of efficiency or performance.

If the asymmetric torque capacity is viewed as a handicap to overcome, the results from the bench top experiments with a single foil suggest one strong motivation for the use of a significant fore-aft component in the limb kinematics of swimming animals. The use of power stroke/recovery stroke kinematics dramatically reduces the torque requirements during the upstroke; power can even be extracted from the fluid during the upstroke with minimal drag penalty. For an animal such as a sea turtle, which for reasons which may have nothing to do with propulsion has limited upstroke power, this is a significant advantage. However, the use of a feathered upstroke can result in 
a $42 \%$ to $59 \%$ drop in thrust from that of a symmetric stroke, and produces significant mean lift, which may not be desired. The introduction of inline motion can make up for a significant amount, although not all, of the lost thrust, while at the same time reducing the mean lift.

In the biomimetic approach to system design, observation and understanding of animal design and behavior is used to suggest new approaches in cases where it is clear that evolution has produced systems which exceed the performance of current technology. It would be naive, however, to assume that evolution produces solutions which optimize the specific metrics of interest even for ostensibly similar functions. The functional requirements of animal limbs may include propulsion, but they are rarely, if ever, entirely limited to propulsion. Experiments with asymmetric foil kinematics can help to clarify the advantages of inline foil motion within the context of asymmetric torque capabilities, and provide a more accurate picture of the cost of asymmetric torque in terms of thrust production and efficiency. 


\section{Chapter 7}

\section{Conclusion}

This thesis documents the design, creation, and successful deployment of "Finnegan the RoboTurtle", an agile and aggressively maneuvering biomimetic autonomous underwater vehicle propelled entirely with biologically inspired oscillating foils. The objective of the work is to prove the ability of rolling and twisting foils to improve the maneuvering performance of AUVs, as defined by the turning radius and turning rate at speed, while simultaneously providing the agility to control six degrees of freedom at low speed in confined space. Through the direct application of experimental observations of sea turtle limb motion during transient maneuvers, we use Finnegan to further demonstrate the power of biomimetics to drive improvements in underwater vehicle design.

\subsection{Harmonically Oscillating Foils}

Foil thrust vectoring capability across widely varying flow conditions produces low speed agility without sacrificing high speed cruising ability. Oscillating foils are capable of vectoring thrust forces within the plane perpendicular to mean foil axis position; when properly powered and sized, thrust vectoring is possible across the entire range of incoming flow speeds experienced during typical AUV operation. With a maximum recorded speed of $1.38 \mathrm{~m} / \mathrm{s}$ (or $0.69 \mathrm{BL} / \mathrm{s}$ ) and maximum yaw rate of $80^{\circ} / \mathrm{s}$, as well as the ability to independently translate in surge and heave, 
the vehicle meets the goal of combining low speed agility with high speed swimming using the same actuator. The high authority thrust and maneuvering forces generated by isolated foils in towing tank and water tunnel tests effectively translate to propulsion of a free swimming vehicle.

Closed loop control of an attitude unstable vehicle with oscillating foils improves vehicle maneuvering performance, as measured by turning radius at speed, by a factor of two. The maneuvering performance achieved during banked and twisting turns, which are particularly appropriate for an attitude unstable foil powered vehicle such as Finnegan, exceeds the best reported performance of existing AUV's by a factor of two. Using control algorithms linear in the modified Rodrigues parameters to support large angle maneuvers, the vehicle is successfully controlled in banked and twisting turns, exceeding the best reported AUV turning performance by more than a factor of two; a minimum turning radius of $0.7 B L$, and the ability to avoid walls detected $>1.8 B L$ ahead, are found for cruising speeds of $0.75 B L / s$, with a maximum heading rate of $40^{\circ} / \mathrm{s}$ recorded.

Foils have been extensively studied experimentally under steady incoming flow conditions with unchanging foil kinematics, allowing the formulation of algorithms for force vectoring using perturbations in just two kinematics parameters for hovering or cruising tasks. The force vectoring algorithms rely on assumptions about the applicability of data collected with unchanging foil kinematics to kinematics varying from stroke stroke.

\subsection{Sea Turtle Maneuvering and Biomimetic Foil Kinematics}

Foil motions adapted from the observed limb kinematics of an adult Green sea turtle can be used to achieve low speed maneuvering performance comparable to that of the turtle. Observations of "Myrtle", a 250kg Green sea turtle (Chelonia mydas) at the New England Aquarium, are detailed; along with steady 
swimming, Myrtle was observed performing $180^{\circ}$ level turns and rapidly actuating pitch to control depth and speed. In other words, adult sea turtles are seen to have the agility to maneuver effectively in search of food within a confined and crowded space. Limb kinematics for the level turning maneuver were replicated by Finnegan, and mean turning rates comparable to those of the turtle $\left(23^{\circ} / s\right)$ were achieved.

Foil thrust production can be increased in situ through the use of kinematics which generate an approximately sinusoidal angle of attack, as demonstrated during a turning maneuver. In adapting turtle limb motions, foil kinematics which produce an approximately sinusoidal nominal angle of attack trace are shown to improve turning performance by as much as $25 \%$; the effect is achieved despite limited knowledge of the flow field..

Thrust production for foils with asymmetric kinematics can be enhanced using bioinspired inline motion. Results from bench top experiments with a single foil suggest a strong motivation for the use of a significant fore-aft component in the limb kinematics of swimming animals. The use of power stroke/recovery stroke kinematics dramatically reduces the torque requirements during the upstroke; power can even be extracted from the fluid during the upstroke with minimal drag penalty. For an animal such as a sea turtle, which for reasons which may have nothing to do with propulsion has limited upstroke power, this is a significant advantage. However, the use of a feathered upstroke can result in a $42 \%$ to $59 \%$ drop in thrust from that of a symmetric stroke, and produces significant mean lift, which may not be desired. The introduction of inline motion can make up for a significant amount, although not all, of the lost thrust, while at the same time reducing the mean lift. 


\section{Bibliography}

[1] B. Allen, R. Stokey, T. Austin, N. Forreser, R. Goldsborough, M. Purcell, and

C. van Alt. Remus: A small, low cost auv; system description, field trials and performance results. 2003.

[2] J. Anderson. Vorticity control for efficient propulsion. PhD dissertation, MIT/Woods Hole Oceanographic Institution, MA, USA, 1996.

[3] Jamie M. Anderson and Narender K. Chhabra. Maneuvering and stability performance of a robotic tuna. Integr. Comp. Biol., 42(2):118-126, 2002.

[4] P.R. Bandyopadhyay, D.N. Beal, and A. Menozzi. Biorobotic insights into how animals swim. Journal of Experimental Biology, 211:206-214, 2008.

[5] D. Beal and P. Bandyopadhyay. A harmonic model of hydrodynamic forces produced by a flapping fin. Experiments in Fluids, 43:675-682, 2007.

[6] D.N. Beal. Propulsion through wake synchronization using a flapping foil. $\mathrm{PhD}$ dissertation, Massachusetts Institute of Technology, Department of Mechanical Engineering, 2003.

[7] R. W. Blake. Fish functional design and swimming performance. Journal of Fish Biology, 65(5):11931222., 2004.

[8] D. M. Boskovic and M. Krstic. Global attitude/position regulation for underwater vehicles. International Journal of Systems Science, 30(9):939-946, September 1999.

[9] O. Cheneval, R.W. Blake, A.W. Trites, and K.H.S. Chan. Turning maneuvers in steller sea lions (eumatopias jubatus). Marine Mammal Science, 23(1):94-109, 2007.

[10] E.G. Drucker and G.V. Lauder. Locomotor forces on a swimming fish: Threedimensional vortex wake dynamics quantified using digital particle image. Journal of Experimental Biology, 202:2393-2412, 1999.

[11] V. I. Fernandez, S. M. Hou, F.S. Hover, J. H. Lang, and M.S. Triantafyllou. Lateral line inspired mems-array pressure sensing for passive underwater navigation. In Proc. of 15th Int. Symp. on Unmanned, Untethered, Submersible Technology, 2007. 
[12] F. Fish, G. Lauder, R. Mittal, A. Techet, M. Triantafyllou, J. Walker, and P. Webb. Construction of a biorobotics AUV based on biological hydrodynamics. In Proc. of 13th Int. Symp. on Unmanned, Untethered, Submersible Technology, 2003.

[13] F. E. Fish. Balancing requirements for stability and maneuverability in cetaceans. Integrative and Comparative Biology, 42(1):85-93, February 2002.

[14] F. E. Fish, M. K. Nusbaum, J. T. Beneski, and D. R. Ketten. Passive cambering and flexible propulsors: cetacean flukes. Bioinspiration and Biomimetics, 1:4248, December 2006.

[15] F.E. Fish. Performance constraints on the maneuverability of flexible and rigid biological systems. In Proceedings of the Eleventh International Symposium on Unmanned Untethered Submersible Technology, pages 394-406.

[16] F.E. Fish. Structure and mechanics of nonpiscine control surfaces. IEEE Journal of Oceanic Engineering, 29(3):605-7621, 2004.

[17] F.E. Fish. A porpoise for power. Journal of Experimental Biology, 208(6):977, 2005.

[18] F.E. Fish, J. Hurley, and D. Costa. Maneuverability by the sea lion zalophus californianus: turning performance of an unstable body design. Journal of Experimental Biology, 206:667-674, 2003.

[19] O. E. Fjellstad and T. I. Fossen. Singularity-free tracking of unmanned underwater vehicles in 6 DOF. In Decision and Control, 1994., Proceedings of the 33rd IEEE Conference on, volume 2, pages 1128-1133, Lake Buena Vista, FL, USA, December 1994.

[20] M. Flores. Flapping motion of a three dimensional foil for propulsion and maneuvering of underwater vehicles. Master of science in ocean engineering, Massachusetts Institute of Technology, MA, May 2003.

[21] T.I. Fossen. Marine Control Systems: Guidance, Navigation and Control of Ships, Rigs and Underwater Vehicles. Marine Cybernetics AS, Trondheim, 2002.

[22] P. Freymuth. Propulsive vortical signatures of plunging and pitching airfoils. In Proceedings of the 26th Aerospace Sciences Meeting, AIAA, 1988.

[23] P. Freymuth. Thrust generation by an airfoil in hover modes. Experiments in Fluids, 9(1):17-24, 1990.

[24] R. Gopalkrishnan, M.S. Triantafyllou, and G.S. Triantafyllou. Active vorticity control in a shear-flow using a flapping foil. Journal of Fluid Mechanics, 274:1-21, 1994. 
[25] J. Gray. Studies in animal locomotion: VI. the propulsive powers of the dolphin. Journal of Experimental Biology, 13:192-199, 1936.

[26] A. Bradley H. Singh, D.Yoerger. Issues in AUV design and deployment for oceanographic research. In Proceedings of the 1997 IEEE International Conference on Robotics and Automation, 1997.

[27] Øyvind Haugsdal. Motion control of oscillating foils for steady propulsion and starting maneuvers. Master of science in ocean engineering, Massachusetts Institute of Technology, MA, 2000.

[28] G.C. Hayes, J.D. Metcalfe, A.W. Walne, and R.P. Wilson. First records of flipper beat frequency during sea turtle diving. Journal of Experimental Marine Biology and Ecology, 303:243-260, 2004.

[29] S. Hoerner. Fluid-Dynamic Drag. Hoerner Fluid Dynamics, Vancouver, Washington, 1965.

[30] F. S. Hover, Ø. Haugsdal, and M. S. Triantafyllou. Effect of angle of attack profiles in flapping foil propulsion. Journal of Fluids and Structures, 19:37-47, January 2004.

[31] Jindong Liu; Huosheng Hu. Mimicry of sharp turning behaviours in a robotic fish. Robotics and Automation, 2005. ICRA 2005. Proceedings of the 2005 IEEE International Conference on, pages 3318-3323, 18-22 April 2005.

[32] M. Isaac, S. Adams, M. He, N. Bose, C. Williams, R. Bachmeyer, and T. Crees. Manoeuvring experiments using the MUNExplorer AUV. In Proc., Underwater Technology 2007, 2007.

[33] P.J. Oxford J. Davenport, Sarah A. Munks. A comparison of the swimming of marine and freshwater turtles. Proceedings of the Royal Society of London, Series B, Biological Sciences, 220(1221):447-475, February 1984.

[34] N. Kato. Control performance in horizontal plane of fish robot with mechanical pectoral fins. IEEE Journal of Oceanic Engineering, 2000.

[35] N. Kato. Median and paired fin controllers for biomimetic marine vehicles. $A p$ plied Mechanics Reviews, 58(4), 2005.

[36] N. Kato, H. Liu, and H. Morikawa. Biology-inspired precision maneuvering of underwater vehicles. In Proc. of 12th Intntl Offshore and Polar Engineering Conf, 2002.

[37] M. Kemp, B. Hobson, J. Janet, and C. Pell. Energetics of the oscillating fin thruster. In Proc. of 13th Int. Symp. on Unmanned, Untethered, Submersible Technology, 2003. 
[38] M. Kemp, B. Hobson, J. Janet, C. Pell, and E. Tytell. Assessing the performance of oscillating fin thruster vehicles. In Proc. of 12th Int. Symp. on Unmanned, Untethered, Submersible Technology, 2001.

[39] M. Kemp, B. Hobson, and Jr. J.H. Long. Madeleine: an agile AUV propelled by flexible fins. In Proc. of 14th Int. Symp. on Unmanned, Untethered, Submersible Technology, 2005.

[40] G. Lauder and B. Jayne. Pectoral fin locomotion in fishes: Testing drag-based models using three-dimensional kinematics. Amer. Zool., 36:567-581, 1996.

[41] G.V. Lauder, P.G. Madden, R.Mittal, H. Dong, and M Boskurttas. Locomotion with flexible propulsors I: Experimental analysis of pectoral fin swimming in sunfish. Bioinspiration and Biomimetics, 1:25-34, 2006.

[42] J.C. Liao, D.N. Beal, G.V. Lauder, and M.S. Triantafyllou. Fish Exploiting Vortices Decrease Muscle Activity. Science, 302:5650, 2004.

[43] S. Licht, V. Polidoro, M. Flores, F. Hover, and M.S. Triantafyllou. Design and projected performance of a Flapping Foil AUV. IEEE Journal of Oceanic Engineering, 29(3):786-794, 2004.

[44] J. Liu, I. Dukes, and H. Huosheng. Novel mechatronics design for a robotic fish. Intelligent Robots and Systems, 2005. (IROS 2005). 2005 IEEE/RSJ International Conference on, pages 807-812, 2-6 Aug. 2005.

[45] J. H. Long, Jr., J. Schumacher, N. Livingston, and M. Kemp. Four flippers or two? Tetrapodal swimming with an aquatic robot. Bioinspiration and Biomimetics, 1:20-29, March 2006.

[46] J.R. Lovvorn. Upstroke thrust, drag effects, and stroke-glide cycles in wingpropelled swimming by birds. American Zoologist, 41(2):154-165, 2001.

[47] C. Martin. Design and performance evaluation of a biomimetic flapping foil. Master of science in naval architecture and marine engineering, Massachusetts Institute of Technology, MA, June 2001.

[48] J.W. Mason, R.; Burdick. Experiments in carangiform robotic fish locomotion. Robotics and Automation, 2000. Proceedings. ICRA '00. IEEE International Conference on, 1:428-435 vol.1, 2000.

[49] K. McCletchie. Force and hydrodynamic efficiency measurements of a threedimensional flapping foil. Master of science in mechanical and ocean engineering, Massachusetts Institute of Technology, MA, May 2004.

[50] K.A. Morgansen., P.A. Vela, and J.W. Burdick. Trajectory stabilization for a planar carangiform robot fish. Robotics and Automation, 2002. Proceedings. ICRA '02. IEEE International Conference on, 1:756-762 vol.1, 2002. 
[51] F.R. O'Keefe and Matthew T. Carrano. Correlated trends in the evolution of the plesiosaur locomotor system. Paleobiology, 31(4):665-675, 2005.

[52] A. Pascoal. The AUV MARIUS: Mission scenarios, vehicle design, construction and testing. In Proceedings of the 2nd Workshop on Mobile Robots for Subsea Environments, 1988.

[53] V. Polidoro. Flapping foil propulsion for cruising and hovering autonomous underwater vehicles. Master of science in ocean engineering, Massachusetts Institute of Technology, MA, May 2003.

[54] P. Prempraneerach, F.S. Hover, and M.S. Triantafyllou. The effect of chordwise flexibility on the thrust and efficiency of a flapping foil. http://web.mit.edu/towtank/www/Papers/FlexibleFoil_Paper.pdf.

[55] D. Read. Oscillating foils for propulsion and maneuvering of ships and underwater vehicles. Master of science in naval architecture and marine engineering, Massachusetts Institute of Technology, MA, February 1999.

[56] Saroj Saimek and Perry Y. Li. Motion planning and control of a swimming machine. The International Journal of Robotics Research 2004, 23:27-53, 2004.

[57] M. Sfakiotakis, D.M. Lane, and J.B.C Davies. Review of fish swimming modes for aquatic locomotion. IEEE Journal of Oceanic Engineering, 24(2):257-252, 1999.

[58] H. Singh, A. Can, R. Eustice, S. Lerner, N. McPhee, O. Pizarro, and C. Roman. SeaBED AUV offers new platform for high-resolution imaging. EOS, Trans. Amer. Geophysical Union, 85(31):289,294295, 2004.

[59] S.M. Smith, P.E. An., K. Holappa, J. Whitney, A. Burns, K. Nelson, E. Heatzig, O. Kempfe, D. D. Kronen, T. T. Pantelakis, E. Henderson, G. Font, R.Dunn, and S.E. Dunn. The Morpheus ultramodular autonomous underwater vehicle. Oceanic Engineering, IEEE Journal of, 26(4):453-465, Oct 2001.

[60] J. Tangorra, S. Davidson, P. Madden, G. Lauder, and I Hunter. A biorobotic pectoral fin for autonomous undersea vehicles. In Proc. 2006 IEEE EMBS, 2006.

[61] A. Techet. Propulsive performance of biologically inspired flapping foils at high Reynolds numbers. Journal of Experimental Biology, 211:274-279, 2008.

[62] G.S. Triantafyllou, M.S. Triantafyllou, and M.A. Grosenbaugh. Thrust development in oscillating foild with application to fish propulsion. Journal of Fluids and Structures, 7(2), 1993.

[63] M. Triantafyllou, A. Techet, and F. Hover. Review of experimental work in biomimetic foils. IEEE Journal of Oceanic Engineering, 29(3), 2004. 
[64] M. S. Triantafyllou and G. S. Triantafyllou. An efficient swimming machine. Scientific American, 272:40-46, 1995.

[65] M.S. Triantafyllou, A.H. Techet, and F.S. Hover. Review of experimental work in biomimetic foils. IEEE Journal of Oceanic Engineering, 29, July 2004.

[66] P. Tsiotras. Stabilization and optimality results for the attitude control problem. Journal of Guidance Control and Dynamics, 19(4):772-779, Jul-aug 1996.

[67] J. Videler and P. Kamermans. Differences between upstroke and downstroke in swimming dolphins. Journal of Experimental Biology, 119(1):265-274, 1985.

[68] M. Watts. Emulating the fast-start swimming performance of the chain pickerel (esox niger) using a mechanical fish design. Master of science in naval architecture and marine engineering, Massachusetts Institute of Technology, MA, September 2006.

[69] P. W. Webb. Maneuverability - general issues. IEEE Journal of Oceanic Engineering, 29(3):547-555, July 2004.

[70] P.W. Webb. The physiology of Fishes, chapter 'Swimming', pages 3-24. CRC Marine Science Series, New York, New York, 1998.

[71] D. Weihs. Stability versus maneuverability in aquatic locomotion. Integrative and Comparative Biology, 42(1):127-134, February 2002.

[72] M. Wolfe. Open loop performance of a biomimetic flapping foil autonomous underwater vehicle. Master of science in ocean engineering, Massachusetts Institute of Technology, MA, December 2006.

[73] M. Wolfe, S. Licht, F. Hover, and M.S. Triantafyllou. Open loop performance of 'Finnegan', the Biomimetic Flapping Foil AUV. In Proc. of 16th Intntl Offshore and Polar Engineering Conf, 2006.

[74] J. Wyneken. The biology of sea turtles, chapter 'Sea turtle locomotion: mechanics, behavior and energetics.', pages 165-198. CRC, Boca Raton, Florida, 1997.

[75] Jeanette Wyneken. Comparative and Functional Considerations of Locomotion in Turtles. PhD dissertation, University of Illinois at Urbana-Champaign, Department of Ecology, Ethology and Evolution, 1988. 